\title{
Analyse von Schälschadensinventuren in Harz und Solling
}

Unter Berücksichtigung ausgewählter Umweltparameter in ihrer Rolle als potentielle Einflussfaktoren

D I S S E R T A T I O N

zur Erlangung des Doktorgrades der Fakultät für Forstwissenschaften und Waldökologie der Georg-August-Universität Göttingen

vorgelegt von

$\mathrm{P}$ H I L I P P $\mathrm{S} \mathrm{C} \mathrm{H} \mathrm{O} \mathrm{M} \mathrm{A} \mathrm{K} \mathrm{E} \mathrm{R}$ Assessor des Forstdienstes, M.Sc.

geboren in Stade

Göttingen, im August 2014 

1. Gutachter:

. Prof. Dr. Stefan Schütz

2. Gutachter:

. Prof. Dr. Niko Balkenhol

Tag der mündlichen Prüfung:

25. Februar 2015 



\section{Vorwort}

Die vorliegende Forschungsarbeit wurde vom damaligen Niedersächsischen Ministerium für Ernährung, Landwirtschaft, Verbraucherschutz und Landesentwicklung an die Abteilung für Forstzoologie und Waldschutz des Büsgen-Instituts der Universität Göttingen in Auftrag gegeben. Unter der Leitung von Prof. Dr. Stefan Schütz war Dr. Ferdinand Rühe, Arbeitsbereich Wildbiologie, mit der Initiierung und Entwicklung des Projektes betraut.

Die Inventurdaten zur Berechnungsgrundlage der Schälschädigung stammen aus den Schälschadenskartierungen von Dr. Oliver Trisl. Die Forsteinrichtungsdaten der Untersuchungsgebiete stellte das Niedersächsische Forstplanungsamt bereit. Die Streckendaten zum Rotwildabschuss im Solling und im Harz wurden durch die örtlichen Forstämter der Niedersächsischen Landesforsten AöR sowie durch die Leitung des Nationalparks Harz übermittelt. Die Berechnungen geographischer Parameter auf der Basis digitaler Geländemodelle erfolgten durch Dr. Rainer Schulz, Abteilung für Forstbiometrie, Büsgen-Institut der Universität Göttingen. Allen sei sehr herzlich gedankt! 



\section{Inhaltsverzeichnis}

1 Einleitung 1

1.1 Vom Schälen zum Schaden . . . . . . . . . . . . . . . . . . . . 1

1.2 Impuls und Ziel der vorliegenden Arbeit $\ldots \ldots \ldots$. . . . . . . 6

1.3 Formulierung von Hypothesen $\ldots \ldots \ldots \ldots$

2 Material und Methoden 11

2.1 Die Untersuchungsgebiete $\ldots \ldots \ldots \ldots \ldots$

2.1 .1 Harz . . . . . . . . . . . . . . . . . . . . . . . 11

$2.1 .2 \quad$ Solling . . . . . . . . . . . . . . . . . . . . . . . . . . 12

2.1 .3 Flächeneinheiten . . . . . . . . . . . . . . . . . . . 13

2.2 Erhebung der abhängigen Variable $\ldots \ldots \ldots \ldots \ldots$

2.2 .1 Inventurverfahren . . . . . . . . . . . . . . . . . . . 13

2.2 .2 Datenumfang und Dateneignung. . . . . . . . . . . . . . 15

$2.2 .3 \quad$ Verwendeter Datenpool . . . . . . . . . . . . . . . . . 18

2.3 Herkunft der unabhängigen Variablen . . . . . . . . . . . . . . . 20

$2.3 .1 \quad$ Quantifizierung vorangegangener Schälschäden . . . . . . . . 20

2.3 .2 Geländespezifische Merkmale $\ldots \ldots \ldots \ldots . \ldots . \ldots 21$

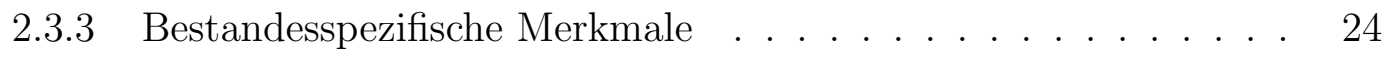

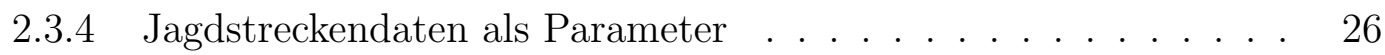

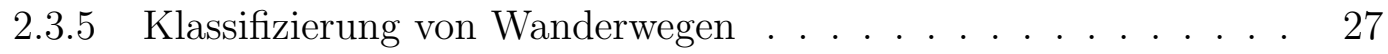

2.4 Instrumente der statistischen Analyse . . . . . . . . . . . . . . . 28

2.4 .1 Statistische Tests und weitere Formeln . . . . . . . . . . 28

2.4 .2 Logistisches Regressionsmodell . . . . . . . . . . . . . . . . . . . . . 29

2.4 .3 Berechnung der Chancenverhältnisse . . . . . . . . . . . . 31

2.4 .4 Generalisierte lineare Modelle . . . . . . . . . . . . . . . . . 33

2.4 .5 Verwendete Software . . . . . . . . . . . . . . . . . . . 33 
3 Charakterisierung der Stichprobenpunkte 35

$3.1 \quad$ Lage der Stichprobenpunkte im Gelände . . . . . . . . . . . . . . 35

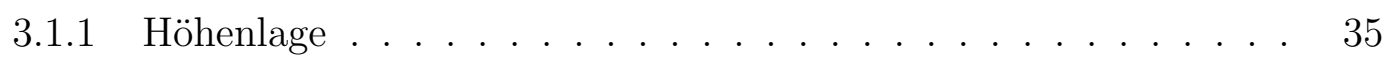

3.1 .2 Hangneigung und -richtung des Geländes . . . . . . . . . . . 35

$3.1 .3 \quad$ Exponiertheit im Gelände . . . . . . . . . . . . . . . . . . . . 41

3.1 .4 Einstrahlungspotential am Stichprobenpunkt . . . . . . . . . . 43

3.2 Bestandesparameter am Stichprobenpunkt . . . . . . . . . . . . . 44

3.2 .1 Bestandesalter. . . . . . . . . . . . . . . . . . . . 44

3.2 .2 Bestandestypen und Baumartenzahl. . . . . . . . . . . 45

3.3 Jagdstreckenaufkommen im Referenzgebiet . . . . . . . . . . . . . 47

$3.3 .1 \quad$ Zeitliche Verteilung der Rotwilderlegungen . . . . . . . . . . . 47

3.3 .2 Räumliche Verteilung der Rotwilderlegungen . . . . . . . . . . . 51

$3.3 .3 \quad$ Beanspruchte Jagdfläche je Revier . . . . . . . . . . . . . . . 53

4 Analyse des Schälschadenaufkommens 55

$4.1 \quad$ Aktuelle und vorangegangene Schälschäden . . . . . . . . . . . . . 55

4.1 .1 Neuschälaufkommen der Inventuren . . . . . . . . . . . . . 55

4.1 .2 Neuschäle an Orten mit Altschälvorkommen . . . . . . . . . . 58

4.2 Schälaufkommen verschiedener Geländeformen . . . . . . . . . . . . . . 61

4.2 .1 Differenzierung nach Höhenlage . . . . . . . . . . . . . . . 61

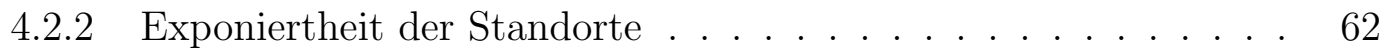

4.2 .3 Hangneigung des Geländes . . . . . . . . . . . . . . . . . 64

$4.2 .4 \quad$ LUV- und LEE-Lage . . . . . . . . . . . . . . . . . . . 67

4.2 .5 Strahlungsinput am Standort . . . . . . . . . . . 68

4.3 Jagdstreckendaten als Indikatoren für Schälschäden . . . . . . . . . . 68

$4.3 .1 \quad$ Korrelation der Schälrate mit Höhe der Jagdstrecke . . . . . . . 68

4.3 .2 Nähe zu Wanderwegen als Kriterium . . . . . . . . . . . . 71

5 Vorbetrachtung der Chancenverhältnisse 73

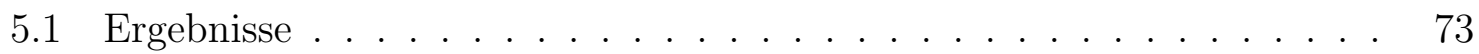

5.1 .1 Altschäle . . . . . . . . . . . . . . . . . . . . . 73

5.1 .2 Kontinuität der Schädigung _ . . . . . . . . . . . . . 74

5.1 .3 Höhenlage . . . . . . . . . . . . . . . . . . . . 75

5.1 .4 Hangneigung . . . . . . . . . . . . . . . . . 75

5.1 .5 Exposition $\ldots \ldots \ldots \ldots \ldots \ldots \ldots \ldots$ 


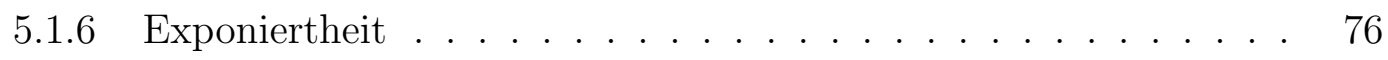

5.1 .7 Strahlung . . . . . . . . . . . . . . . . . . . 77

5.1 .8 Bestandesalter. . . . . . . . . . . . . . . . . . . . . . . 78

5.1 .9 Baumartenzahl . . . . . . . . . . . . . . . . . 78

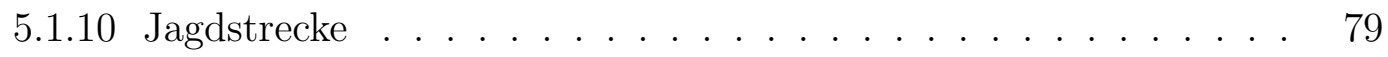

5.1 .11 Wegeklasse $\ldots \ldots \ldots \ldots \ldots$. . . . . . . . . 80

5.2 Zusammenfassung $\ldots \ldots \ldots \ldots \ldots \ldots \ldots$

6 Binäre logistische Regressionsmodelle 83

8.1 Ergebnisse $\ldots \ldots \ldots \ldots \ldots$

6.1 .1 Inventurjahre 2002, 2004 und 2006 im Harz . . . . . . . . . 84

6.1 .2 Inventurjahre 2002, 2003 und 2006 im Solling . . . . . . . . 87

6.1 .3 Gesamtmodell . . . . . . . . . . . . . . . . . . . . . 90

6.2 Zusammenfassung . . . . . . . . . . . . . . . . . . . . . . . . . . . . 91

7 Generalisierte Lineare Modelle 93

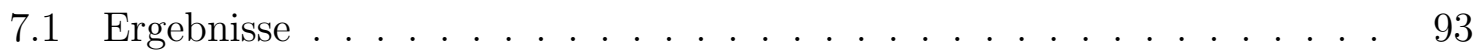

7.1 .1 Gesamtmodell . . . . . . . . . . . . . . . . . . . . 94

7.1 .2 Gebietsweise Analyse . . . . . . . . . . . . . . . . . . . 95

7.1 .3 Analyse der Inventurjahre je Gebiet . . . . . . . . . . . . . 97

7.1 .4 Modellierung nach Parametergruppen . . . . . . . . . . . . . . 103

7.2 Zusammenfassung . . . . . . . . . . . . . . . . . 106

8 Konsquenzen für die Hypothesen 107

9 Diskussion 111

9.1 Betrachtung der Voruntersuchung . . . . . . . . . . . . . . . . . 111

9.1 .1 Konsequenzen für die eigene Analyse . . . . . . . . . . . . 112

9.2 Betrachtung der eigenen Analyse $\ldots \ldots \ldots \ldots \ldots \ldots$

$9.2 .1 \quad$ Bewertung der Datengrundlage . . . . . . . . . . . . . 114

9.2 .2 Bewertung der Ergebnisse . . . . . . . . . . . . . . . . 118

9.3 Konsequenz und Ausblick $\ldots \ldots \ldots \ldots$

$\begin{array}{ll}10 \text { Zusammenfassung } & 131\end{array}$ 
$\begin{array}{ll}11 \text { Summary } & 133\end{array}$

Anhang A A

Abbildungsverzeichnis $\quad$ C

Tabellenverzeichnis $\quad$ E

Literaturverzeichnis E 


\section{Einleitung}

\subsection{Vom Schälen zum Schaden}

Rotwild (Cervus elaphus) besitzt die Fähigkeit, sich auf kalkulierbare Störungen, beispielsweise auf Truppenübungsplätzen mit kontinuierlichem militärischem Betrieb, einzustellen. Auch verhält es sich, wenn die lokale Population den Menschen nie als „Beutegreifer" kennengelernt hat, diesem gegenüber weitgehend tolerant (SCHUlTz \& BAILEY 1978, BORKOWSKI 2001). In den Verhältnissen unserer Landschaft reagiert es jedoch äußerst sensibel auf anthropogene Störungen (Altman 1958, Behrend \& Lubeck 1968, Boyle \& SAmson 1985, Jeppesen 1987). Und so führte die zunehmende Präsenz des Menschen in seinem ursprünglichen Lebensraum dazu, dass es sich aus der offenen Landschaft in den Schutz des Waldes zurückzog. Dieser bietet zwar ausreichend Deckung zur Feindvermeidung, doch als Intermediärtyp (Hofmann \& SteWART 1972) trifft das Rotwild im Wald meist nur auf suboptimale Äsungsbedingungen (VÖLK 1999, LiCOPPE 2006).

Dabei ist Rotwild bei der Nahrungssuche nicht gerade wählerisch - PETRAK (1982) zeigte, dass mehr als $80 \%$ der erreichbaren Pflanzenarten eines Gebietes vom Rotwild auch zur Äsung genutzt werden und andere Autoren berichten von noch höheren Anteilen des vorhandenen Spektrums (KRAus 1984). So wurden bereits 300 Pflanzenarten als Äsung für das Rotwild nachgewiesen (DzIECIOLOWSKI ET AL. 1969). Abhängig von der Jahreszeit macht auch die Äsung an Gehölzen einen Teil der Nahrungsaufnahme aus (DAUSTER 1939). Im speziellen Fall der Schäle entfernt das Rotwild die Rinde vom Stamm der Bäume. Hierzu treibt das Rotwild seine unteren Schneidezähnen in die Rinde und stützt die Bewegung mit dem Oberkiefer am Stamm ab. Während im Winter so nur ein Benagen möglich ist, können während der Vegetationszeit ganze Streifen der Rinde vom Baum gerissen werden.

Dass das Rotwild zumindest einen Teil der geschälten Baumrinde aufnimmt, zeigen diverse Nachweise durch Pansenuntersuchungen (JEnsen 1968, SEnn 1987, PiCARD 
et Al. 1991, Groot Bruinderink \& Hazebroek 1995, van de Veen 1979, Welch et Al. 1987, Latham et Al. 1999, Mitchell \& McCowan 1986). Auch über die Gründe hierfür wurde bereits viel geforscht. Zunächst ist festzustellen, dass Rotwild seine Eigenschaft als intermediärer Typ Pflanzenfresser offenbar für sich nutzen kann, indem es bei der Nahrungswahl flexibel auf seine Umweltbedingungen reagiert (Hofmann 1989, Gebert \& Verheyden-Tixier 2001). Dies zeigt sich beispielsweise im Verlauf der Jahreszeiten, wenn auch das Nahrungsangebot in Qualität und Verfügbarkeit wechselt (VAn De Veen 1979, Bugalho \& Milne 2003).

In Phasen knapper Nahrung wird daher tendenziell mehr Baumrinde vom Rotwild aufgenommen als in der übrigen Zeit (Ahlen 1965, Dzieciolowski 1967, BorowSKI \& KossaK 1975, Ueda ET AL. 2002), wobei hohe Schneelagen im Winter diese Tendenz entsprechend verstärken können (JAMrozy 1980). So liegt die Vermutung nahe, dass Rinde im Vergleich zu anderen verfügbaren Nahrungsquellen solcher Phasen einen höheren Nährstoff- oder Energieinput liefert. Untersuchungen von RADEMACHER (2005) zeigten zunächst, dass die Rinde diverser Baumarten im Vergleich zu Blättern und Nadeln weniger Proteine, Phosphor und Kalium enthält und dass der Anteil an Magnesium und Calcium in etwa auf selbem Niveau oder etwas darüber liegt. Im Vergleich zum Holz der Äste und Zweige wurde jedoch deutlich, dass die Rinde in diesem Fall erheblich mehr von allen diesen Nährstoffen bietet. In einer Studie zum Schälverhalten des Elches wurde speziell für die Rinde der Fichte ein höherer Calciumgehalt bei zugleich geringerem Faseranteil festgestellt als für andere Baumarten der betrachteten Äsungsfläche (RANDVEer \& HEIKKIL ̈̈ 1996). Mehrheitlich werden solche Unterschiede allerdings als marginal betrachtet, denn auch für viele andere Baumarten wurden bereits vergleichbare Werte ermittelt und Schälschäden im Winter hieran dokumentiert (Faber 1996, Ando et Al. 2003, Danell et Al. 2003, Kuiters et AL. 2006, TAmura \& OHARA 2005). So ist Rinde in Zeiten knapper Ressourcen offenbar generell als ausreichende Nahrungsquelle und verhältnismäßig beste Alternative für das Rotwild anzusehen (GILL 1992).

Ein anderer Aspekt, der für die Aufnahme von Baumrinde sprechen könnte, begründet sich in deren potentiellen Wirkung gegen Parasitenbefall. Diese Hypothese wurde aufgrund der Beobachtung aufgestellt, dass sich die Schälaktivität weiblichen Rotwildes alljährlich zur Setz- und zur anschließenden Laktationszeit erhöhte. Die in der Rinde enthaltenen Terpene und Tannine werden damit in Zusammenhang gebracht, die Alttiere in dieser für sie und den Nachwuchs sensiblen Zeit vor überhöhtem Befall 
mit Endoparasiten zu schützen (Hoste ET AL. 2006). Denn hohe Befallsraten nehmen nachweislich direkten Einfluss auf die Konstitution der Alttiere und damit auch indirekt auf den Erfolg der Reproduktion (Mulvey ET AL. 1994). Gerade in dieser kritischen Phase des Jahres (LOUDON 1985) sollten Infektionen also tunlichst vermieden werden (Møller et AL. 1993). Darüber hinaus könnte die Zufuhr von Tanninen beim Rotwild generell dazu führen, dass Proteine besser über das Verdauungssystem in den Körper aufgenommen werden und die Verwertung der vorhandenen Nahrung somit optimiert wird (Min ET AL. 2003). Hier ließe sich auch für die Hirsche ein evolutionärer Vorteil im Schälen von Rinde erkennen. Denn wenn man deren Reproduktionerfolg während der Brunft unter anderem vom vorherigen Erreichen hoher Körpergewichte (Clutton-Brock et Al. 1982, Clutton-Brock 1988) und der allgemeinen Funktionsfähigkeit des Immunsystems während der Brunft abhängig macht, könnten die aus der Rinde gewonnenen Substanzen möglicherweise den entscheidenden Vorteil hierfür erbringen.

\section{Einfluss des Schalenwildes auf den Wald}

Die Anwesenheit wiederkäuender Schalenwildarten kann sich generell erheblich auf das Verjüngungsgeschehen und die Baumartenzusammensetzung im Wald auswirken (vgl. Ammer 1996, Akashi \& Nakashizuka 1999, Gill 2000, Gill \& Beardall 2001, Danell et AL. 2003, Gill 2006) und zu langfristigen Beeinträchtigungen führen (TAnentzap ET AL. 2011 und 2012, White 2012). Gerade die Verjüngung der Laubholzarten hat kaum eine Chance, „aus dem Äser“ zu wachsen, wenn der Verbiss durch erhöhte Schalenwildbestände permanent und konzentriert wirkt (KUITERS \& SLIm 2002). Während bei moderat erhöhtem Verbissdruck zunächst noch mit einer Entmischung der Baumarten zu rechnen ist (PUTMAn 1986), da insbesondere das Rehwild (Capreolus capreolus) als Konzentratselektierer (Hofmann \& StewarT 1972) vornehmlich die begehrten Arten wie Eiche (Quercus spec.), Ahorn (Acer spec.), Esche (Fraxinus excelsior) und Tanne (Abies alba) beäst, kann das Zusammentreffen mehrerer ungünstiger Faktoren bei extremem Schalenwildvorkommen sogar bis zur Devastierung von Waldstandorten führen (Yokoyama ET AL. 2001, Ando ET AL. 2006).

Obwohl die Populationen der Schalenwildarten innerhalb Europas in den letzten Jahrhunderten stark angestiegen sind (GILL 1992, KuITERs ET AL. 1996, LOISON Et AL. 2003, Gordon et AL. 2004, WArd 2005, Milner et AL. 2006), dürften solche Vorfälle jedoch zu den absoluten Ausnahmen zählen. Doch wie hoch ist der Ef- 
fekt wildlebender Pflanzenfresser auf die Umwelt? Quantitative Untersuchungen hierzu sind rar. Umso interessanter sind die Werte, die PERsson ET AL. (2000) auf der Basis von veröffentlichten Aktivitätsdaten beobachteter Elche (Alces alces) hochrechneten. Demzufolge nimmt ein durchschnittlicher Elch etwa $8.000 \mathrm{~kg}$ grünes Pflanzenmaterial pro Jahr auf, wobei er nebenbei noch einen Hektar Fläche platttrampelt und 5.000 Kothaufen produziert. Die Vorstellung dieser so nüchtern zusammengetragenen Fakten mag zum Schmunzeln anregen, verdeutlicht aber auch, welches Potential in hohen Populationsdichten großer Pflanzenfresser steckt. Je nach Ausmaß müssen Verbiss und Schäle also als bedeutende dynamische Faktoren im Wald gelten.

\section{Bedeutung als Schaden}

Während der Einfluss durch Verbiss in den ersten Entwicklungsstadien eines Baumes greift, entsteht durch das Schälen der Rinde ein meist letaler Schaden am noch jungen, aber bereits etablierten Baum. Hierbei sind insbesondere die Folgeschäden von Bedeutung, denn durch das mechanische Entfernen der Rinde entstehen Eintrittspforten, die es holzzersetzenden Pilzen ermöglichen, in den bis dahin gesunden Baum einzudringen. Es kommt zu Fäuleerscheinungen, die an der Fichte (Picea abies) vor allem durch den Blutenden Schichtpilz (Stereum sanguinolentum) hervorgerufen werden (SCHÖNHAR 1975, Randveer \& Heikkil ̈̈ 1996, Vasiliauskas \& Stenlid 1998)!1Die Fichte gilt dabei als „bevorzugt geschälte“ Baumart (UECKERMANN 1960) und wird sowohl im Sommer als auch im Winter geschält. An der Buche lassen sich hingegen fast ausschließlich Sommerschälschäden finden, welche während der Zeit des Saftflusses verursacht wurden (Ueckermann 1956, De Crombrugghe 1965, Müller 1979).

Für das Bestandesgefüge ergibt sich aus den geschilderten Zusammenhängen der Schäleinwirkung eine eigene Dynamik, denn die durch Fäule geschädigten Bäume zeigen eine Prädisposition gegenüber weiteren Beeinträchtigungen. So unterliegen geschälte Bäume in der Regel einem höheren Schnee- sowie Sturmbruchrisiko, wobei das Risiko bei größerer Abmessung der Schälwunde und geringer Dimension des Baumes steigen soll (Kramer \& Womelsdorf 1985, Otтo 1985, Borchers 1964, zitiert in Moog 2008). In Naturwäldern können solche Effekte zunächst als wertneutral betrachtet werden. In Wirtschaftswäldern hingegen verursacht bereits der Pilzbefall am

\footnotetext{
${ }^{1}$ An der Buche (Fagus sylvatica) vornehmlich durch Zunderschwamm (Fomes fomentarius) (VANIK 1979, Kim 1983) und an der Esche durch Phaeoacremonium sp. (VASILIAUsKas \& STENLID 1998).
} 
noch stehenden und lebenden Baum einen Schaden, der über die verminderten Erlöse beim Holzverkauf auch spürbare Ertragseinbußen für den Forstbetrieb mit sich bringt.

Die mittlere Längsausbreitung der Fäule im Stamm wird in der Literatur zumeist mit 2 bis 4 Metern angegeben (in Ausnahmen deutlich mehr), wobei manche Autoren einen linearen bis degressiven Fortgang des Fäuleprozesses voraussetzen (BEISEL 1973, Richter 1974), während andere von einer Stagnation nach Abschluss der Überwallung der Schälwunde ausgehen (LÖFFLER 1975). Da sich die Schälwunde stets im unteren Stammbereich befindet und damit auch der Fäuleprozess im Erdstammstück beginnt, wird zudem von Beginn an der ertragreichste Abschnitt des Stammes in seinem Wert herabgesetzt. Verständlich also, dass die ökonomischen Auswirkungen von Schälereignissen in vielen Arbeiten im Vordergrund stehen (neben den zuvor genannten z.B. bei Kato 1969, Roeder 1970, Knigge 1975, Bencze 1977, Vasiliauskas 2001 und SCHALLER 2002).

Die monetäre Bewertung von Schälschäden soll an dieser Stelle jedoch nicht vertiefend behandelt, sondern lediglich in ihren Ansätzen betrachtet werden. Die hierzu entwickelten gängigen Verfahren werden z.B. durch Moog (2008) sehr übersichtlich dargestellt. Beginnend mit den Schälschadenstabellen von KATO (1981, 1983) und Kroth ET AL. (1984) stellt er die hierauf aufbauenden Schemata von Binder (1991) und BüCKING ET AL. (2006) vergleichend vor. Die Berechnung der Schadenshöhe bedient sich darin in der Regel dreier Instrumente: Es liegen Wuchsmodelle auf Basis der baumartenspezifischen Ertragstafeln zugrunde, marktübliche Holzerlöse nach gängigen oder aber selbst erarbeiteten Sortierkriterien (da je nach Ansatz auch die Größe der Schälwunde hierbei berücksichtigt wird) sowie ein kalkulatorischer Zinsfuß.

Mit dem Berechnungsschema von Schaller (2002) stellt Moog (2008) schließlich ein Verfahren vor, in dem außerdem der Entnahmezeitpunkt des geschädigten Baumes im einzelnen und die Bestandesentwicklung als Ganzes Einfluss auf die Bewertung des konkreten Schälschadens nehmen. So setze Schaller voraus, dass Bäume der Kraft'schen Klassen 4 und 5 (KrAfT 1884, zitiert nach Burschel \& Huss 1987) aufgrund mangelnder Bedeutung für das Bestandesziel grundsätzlich nicht als schadensfähig gelten könnten und somit in der Bilanzierung auch keine Berücksichtigung fänden. Die Schadensbewertung aller anderer geschälter Bäume wiederum sei abhängig vom Stammdurchmesser des geschädigten Baumes und seiner Distanz zum nächsten ungeschälten Baum, da diese Kriterien im Modell ausschlaggebend für den Zeitpunkt seiner Entnahme seien. Moog beurteilt dieses Verfahren insgesamt positiv. Er hebt 
dabei die Möglichkeit der Senkung von Schadensbilanzen hervor und betont die freie Wahl des Zinsfußes und die fortschrittliche Verwendung von Wachstumsfunktionen. Leider sei das hierfür notwendige Rechenprogramm jedoch nicht frei verfügbar und somit für Sachverständige bei der Schadensherleitung nicht praktisch anwendbar. Dass sich die Schälaktivität von Rotwild im Wald schnell zum erheblichen wirtschaftlichen Schaden entwickelt, wird anhand der intensiven Bemühungen um dessen Bewertung deutlich. Doch warum schält das Rotwild an manchen Orten mehr als an anderen?

\subsection{Impuls und Ziel der vorliegenden Arbeit}

Die vorliegende Studie ist aus einem Projekt hervorgegangen, das vom Niedersächsischen Ministerium für Ernährung, Landwirtschaft, Verbrauchersschutz und Landesentwicklung bereits vor Beginn dieser Arbeit beim damaligen Institut für Forstzoologie und Waldschutz der Fakultät für Forstwissenschaften und Waldökologie der GeorgAugust-Universität Göttingen in Auftrag gegeben worden war. Ziel des Projektes war es, kausale Zusammenhänge zwischen Umweltfaktoren und dem Auftreten von Schälschäden zu detektieren und mittels multivariater Modelle in ihrem Effekt zu quantifizieren. Hierzu sollten die Inventurdaten, welche zur Ermittlung der Schälprozente auf den Landeswaldflächen im Solling und im Harz periodisch erhoben worden waren, als Basis für die Erstellung der abhängigen Variable dienen. Es konnte auf einen Datenpool aus je drei Jahrgängen pro Gebiet zurückgegriffen werden, sodass insgesamt Informationen aus sechs Schälschadenskartierungen vorlagen.

Als mögliche Einflussfaktoren sollten Jagdstreckendaten beider Gebiete in die Modelle eingehen. Darüber hinaus wurden Daten der Forsteinrichtungswerke zur Analyse bereitgestellt. Zusätzlich sollten, mittels geographischer Informationssysteme, Geländeinformationen generiert und ebenfalls in die Modelle eingepflegt werden. Weiterhin war die Berücksichtigung von Daten der lokalen Wetterstationen geplant, um Informationen über die mittleren Schneehöhen an den Stichprobenpunkten zu errechnen, damit diese dann in gleicher Weise als potentielle Einflussgrößen in den Modellen überprüfen werden könnten.

In der ersten Phase des Projektes führte die damalige Arbeitsgruppe des Instituts bereits aus einem Teil der Daten eine Berechnung für das Gebiet des Harzes durch und stellte die Ergebnisse ihres multivariaten Modells in Form einer Voruntersuchung der Öffentlichkeit vor (KIfFneR ET AL. 2008). Aufgrund eines Personalwechsels beginnt 
die hier vorliegende Arbeit mit dem Einstieg in jener Phase des Projektes. So wurde zur Orientierung für die Weiterführung des Vorhabens auf die bereits veröffentlichten Ergebnisse der Voruntersuchung verwiesen, in denen kausale Zusammenhänge zwischen Umweltfaktoren und Schälrate inzwischen festgestellt und diskutiert worden waren. Anhand dieser Richtschnur sollten die dort formulierten Erkenntnisse nun mit der Gesamtheit des Datenpools überprüft und auf breiter Basis weiter verifiziert werden.

Im Rahmen des verbliebenen Projektzeitraumes wurden die Daten in der vorgegebenen Weise analysiert und in binäre logistische Regressionsrechnungen überführt. Die hieraus für beide Untersuchungsgebiete generierten Ergebnisse aller vorliegenden Jahrgänge wurden dem Ministerium in Form eines Arbeitsberichtes übergeben, womit das Projekt offiziell zum Abschluss gelangte.

Aus dem Antrieb heraus, die sehr komplexen multivariaten Modelle zunächst auf breiterer Basis zu erschließen, wurde im Folgenden an dem Projekt weitergearbeitet. Zudem bot der Abschlussbericht, aufgrund seiner formalen Beschränkungen, wenig Einblicke in die bei der Aufbereitung der Daten zu lösenden Probleme. Im Hinblick auf weitere wissenschaftliche Forschung zur Thematik der Schälschadensanalysen und darauf, dass es sich ja um eine angewandte wissenschaftliche Arbeit mit angestrebter Praxisrelevanz handelt, sollten diese Aspekte aber nicht vorenthalten bleiben.

So ist es das Ziel der hier vorliegenden Arbeit, zunächst die Datengrundlage detailliert darzustellen, um über die in den Untersuchungsgebieten vorherrschenden Verhältnisse und insbesondere die Bedingungen an den Stichprobenpunkten eingehend zu informieren. Durch die dann folgende einfache Statistik sollen klare Verhältnisse der einzelnen Parameter geschaffen werden, da die Interpretation der später verwendeten multivariaten Regressionsmodelle ja durchaus nicht als trivial anzusehen ist. So zeigten sich für die darin eingeschlossenen Variablen, je nach Kombination mit anderen eingehenden Parametern, mitunter vollkommen unterschiedliche Effektrichtungen und -größen. Am Ende solcher Projekte entscheidet man sich jedoch letztlich für das Modell mit der höchsten Anpassungsgüte und baut seine inhaltlichen Aussagen hierauf auf. Bei der Beurteilung solcher Ergebnisse sollen deshalb die vorangestellten einfachen statistischen Zusammenhänge eine Orientierung bieten, um die Aussagen der erstellten Modelle möglicherweise neu bewerten zu können. 


\subsection{Formulierung von Hypothesen}

Für die Durchführung dieser Untersuchung werden Arbeitshypothesen aufgestellt, die sich mit den Erwartungen des ursprünglichen Projekts weitgehend decken und anhand derer das vorliegende Datenmaterial aufbereitet und analysiert werden soll. Die Hypothesen orientieren sich auch an den eingangs vorgestellten internationalen Arbeiten, die sich ebenfalls der Frage nach kausalen Zusammenhängen zwischen Schälrate und Umweltbedingungen widmen.

Es wird also zunächst vorausgesetzt, dass eine Verschneidung von Daten aus Schälschadensinventuren, Forsteinrichtungswerken, geographischer Modellierung, Klimastationen und aus Jagdstreckenstatistiken dazu geeignet ist, eine Analyse zu Einflussfaktoren auf die Schälaktivität von Rotwild durchzuführen und Aussagen über die Schälgefährdung von Beständen zu treffen.

\section{Hypothese I}

Punkte mit aktueller Schälschädigung sind auch Orte mit hoher Wiederholungsrate in der Schälschädigung.

In diesem Sinne soll untersucht werden, ob sich eine lokale Kontinuität in der Schälschädigung abzeichnet und ob dass das Vorhandensein von Altschälschäden am Stichprobenpunkt auf neue Schälschäden schließen lässt.

\section{Hypothese II}

Die Höhe der Rotwildjagdstrecke eines Revieres korreliert mit der Höhe des Schälschädigungsgrades im selben Gebiet.

\section{Hypothese III}

Stichprobenpunkte in reich strukturierten Beständen mit mehreren Baumarten weisen ein geringeres Schälrisiko auf als solche in gleichaltrigen Reinbeständen.

\section{Hypothese IV}

An Stichprobenpunkten tieferer Lagen ist die Wahrscheinlichkeit eines Winterneuschälschadens größer als an jenen höherer Lagen. Daneben steigt die Wahrscheinlichkeit in 
strahlungsintensiven und in windabgewandten Lagen.

Lassen sich anhand von Reliefausprägungen Unterschiede in der Intensität von Schälschäden feststellen? Ausgehend vom individuellen Einfluss durch Wind, Temperatur, Niederschlag, Schneelage und Strahlung auf die mikroklimatischen Verhältnisse am Aufnahmepunkt könnte man vermuten, dass das Rotwild im Winter witterungsgeschützte Lagen bevorzugt. Mutmaßlich wird das Rotwild an diesen Standorten mehr Zeit verbringen und somit häufiger in den dortigen Beständen schälen. Die als Indikatoren ermittelten Werte der Höhenlage, Exposition, Hangneigung und Exponiertheit sowie des Einstrahlungspotentials des Geländes sollen hierzu hinsichtlich ihrer Rolle als Einflussfaktoren untersucht werden. 



\section{Material und Methoden}

\subsection{Die Untersuchungsgebiete}

\subsubsection{Harz}

Als erstes Gebiet für die exemplarische Untersuchung der Umweltvariablen wurde der niedersächsische Teil des Harzes herangezogen (vgl. Abb. 2.1). Die zugrunde liegende Fläche umfasst etwa $749 \mathrm{~km}^{2}$ Staatswald (97\% Holzbodenfläche). Auf dem überwiegenden Teil ( $80 \%$ der Fläche) erfolgt eine forstliche Bewirtschaftung durch die vier Forstämter Clausthal, Lauterberg, Riefensbeek und Seesen der Niedersächsischen Landesforsten Anstalt öffentlichen Rechts. Die restlichen $20 \%$ entfallen auf die Verwaltung des Nationalparks Harz. Durch die gesonderten Zielvorgaben innerhalb des Nationalparks sind etwa $10 \%$ der Gesamtfläche dieses Untersuchungsgebietes von der forstwirtschaftlichen Nutzung ausgenommen (AlBers ET AL. 2004). Die Analyse und Darstellung der Ergebnisse auf Basis der Forstamts- oder Reviergrenzen erfolgt anonymisiert.

Mit einem Anteil von $70 \%$ nimmt die Fichte (Picea abies) eine dominierende Stellung unter den Wirtschaftsbaumarten ein. Im Zuge von Waldumbaumaßnahmen ist die Buche (Fagus sylvatica) in einer Zunahme an Flächenanteilen begriffen und erreicht derzeit einen Wert von 21 \%. Neben anderen Laubholz- (6 \%) und Nadelholzarten (3\%) kommen Erle (Alnus glutinosa), Bergahorn (Acer pseudoplatanus), Esche (Fraxinus excelsior), Lärche (Larix decidua) und Douglasie (Pseudotsuga menziesii) vor (BADERSCHNEIDER UND BÖCKMANN 2004).

Die Höhe der Stichprobenpunkte der Schälinventur reicht von 232 bis $894 \mathrm{~m}$ über Normalnull (NN). Eine lange Winterperiode mit hohen Niederschlagswerten sowie eine kurze Frühlingsperiode charakterisieren das Klima im Harz. Wenige Sonnentage und häufiges Aufkommen von Nebel sind typisch für das Wetter. Westliche Winde sind üblich, doch wird die Windrichtung in hohem Maße durch die Topographie des Geländes 


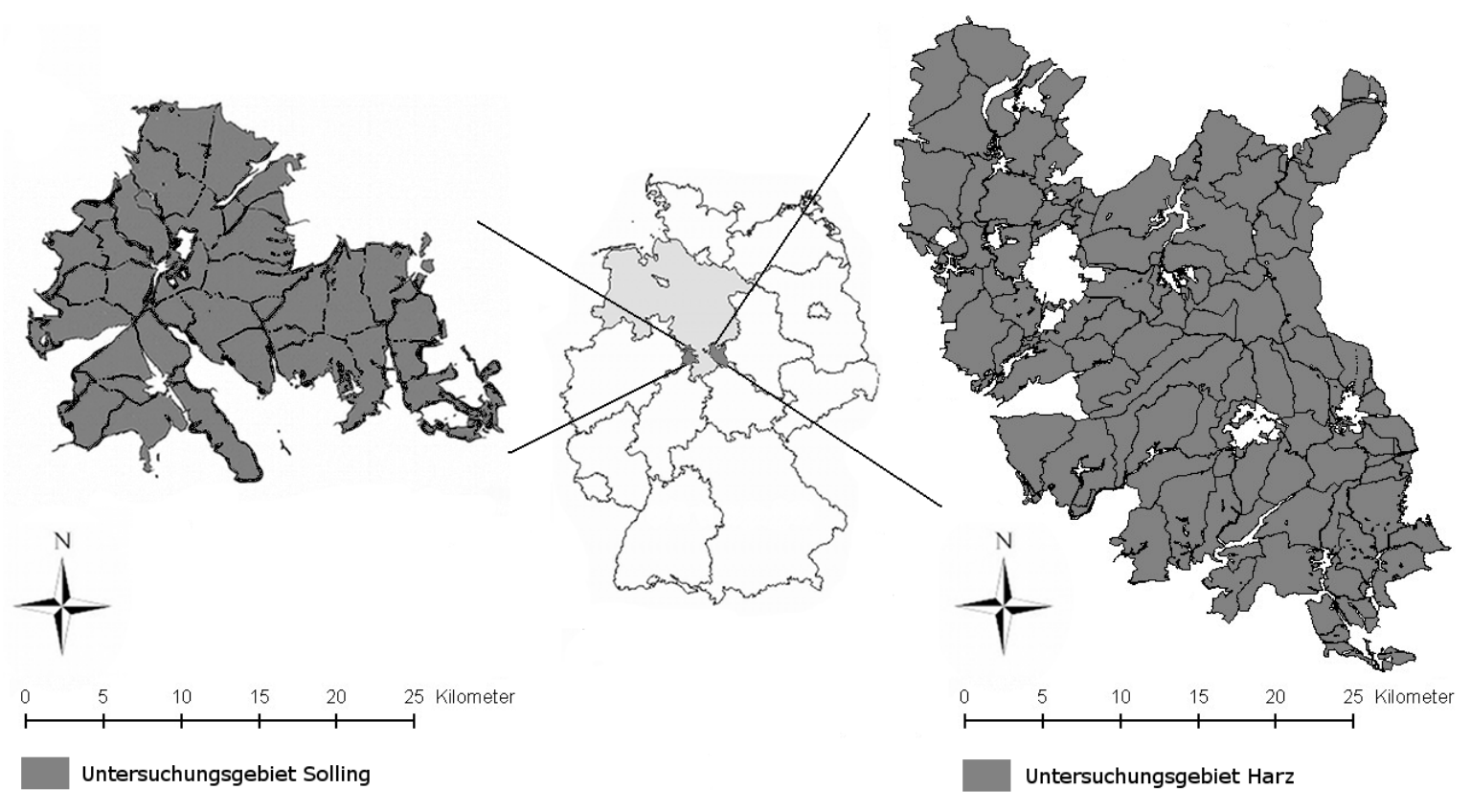

Abbildung 2.1: Lage der Untersuchungsgebiete Solling und Harz im südniedersächsischen Teil Deutschlands und Detaildarstellung der Gebiete mit ihren Teilgebieten

beeinflusst (MATschullat ET AL. 1994). Im Vergleich zu ähnlichen Höhenzügen, gilt der Harz als das kältestes Mittelgebirge Deutschlands (SchwiEterT 1989).

\subsubsection{Solling}

Das zweite Untersuchungsgebiet befindet sich im Solling. Als Teil des Weserberglandes umfasst es etwa $341 \mathrm{~km}^{2}$ Waldfläche (vgl. Abb. 2.1). Es handelt sich ebenfalls um eine Landeswaldfläche, die durch die Niedersächsischen Landesforsten Anstalt öffentlichen Rechts bewirtschaftet wird. Die Buche (Fagus sylvatica) ist hier die dominante Baumart und bestimmt etwa $50 \%$ der Bestandestypen. Die Fichte (Picea abies) steht, mit einem Anteil von etwa $40 \%$, an zweiter Stelle. Neben Buche und Fichte kommen vor allem Eiche (Quercus spec.), Lärche (Larix decidua), Douglasie (Pseudotsuga menziesii) und Kiefer (Pinus sylvestris) als Wirtschaftsbaumarten vor. Charakterisiert wird der Solling darüber hinaus durch das Vorkommen verschiedener anderer Laubholzarten. Mit Werten zwischen 127 and 525 m über NN liegen die Stichprobenpunkte in einem weiten Bereich verschiedener Höhenlagen. 
Tabelle 2.1: Flächengrößen der einzelnen Bezugsebenen, alle Flächenangaben in Hektar; Quelle: Forsteinrichtungsdaten des Niedersächsisches Forstplanungsamtes, Wolfenbüttel

\begin{tabular}{lrrrrrr}
\hline Ebene & Anzahl & Fläche $(\bar{x})$ & StAbw & min & max & Median \\
\hline Gebiet I Harz & & & & & & \\
$\quad$ Forstamt & 5 & 13.990 & 1.200 & 12.486 & 15.316 & 14.221 \\
Revierförsterei & 51 & 1.372 & 232 & 509 & 1.809 & 1.386 \\
Abteilung & 3.649 & 19,2 & 6,4 & 2,4 & 50,7 & 18,7 \\
Unterabteilung & 7.606 & 9,2 & 6,70 & 0,1 & 45,9 & 7,6 \\
Gebiet II Solling & & & & & & \\
Forstamt & 3 & 12.688 & 1.565 & 10.531 & 14.199 & 13.333 \\
Revierförsterei & 30 & 1.269 & 112 & 975 & 1.458 & 1.308 \\
Abteilung & 1.885 & 20,2 & 7,2 & 0,6 & 57,5 & 19,6 \\
Unterabteilung & 4.270 & 8,9 & 6,9 & 0,1 & 42,4 & 7,0 \\
\hline
\end{tabular}

\subsubsection{Flächeneinheiten}

Aufgrund der vorliegenden Datengrundlage, die auf den Verwaltungsgrenzen der Niedersächsischen Landesforsten A.ö.R. aufbaut, werden die Flächenbezüge in jener Form beibehalten. Die oberste Untersuchungsebene stellen die Gebiete dar, welche in diesem Fall durch die Waldgebiete des Solling und des westlichen Teils des Harzes repräsentiert werden. Beide Gebiete untergliedern sich jeweils in Teilgebiete, deren Grenzlinien denen der Forstämter entsprechen. Mit den Revierförstereien steht ein Flächenbezug zur Verfügung, der mit einer durchschnittlichen Ausdehnung von $14 \mathrm{~km}^{2}$ eine mittlere Projektionsebene bietet. Eine weitere Fragmentierung ergibt sich durch die Aufteilung in Abteilungen, Unterabteilungen und die Unterfläche. Tabelle 2.1 enthält die mittleren Flächengrößen der aufgezählten Bezugsebenen mit Ausnahme der niedrigsten Gliederungsebene.

\subsection{Erhebung der abhängigen Variable}

\subsubsection{Inventurverfahren}

Als Grundlage der Analyse wurde auf die Ergebnisse von Schälschadenskartierungen zurückgegriffen, die in beiden Untersuchungsgebieten für jeweils drei Perioden vorlagen (vgl. Tab. 2.2, S. 16). Die Inventuren folgten der Methode nach TRISL 1998, bei 


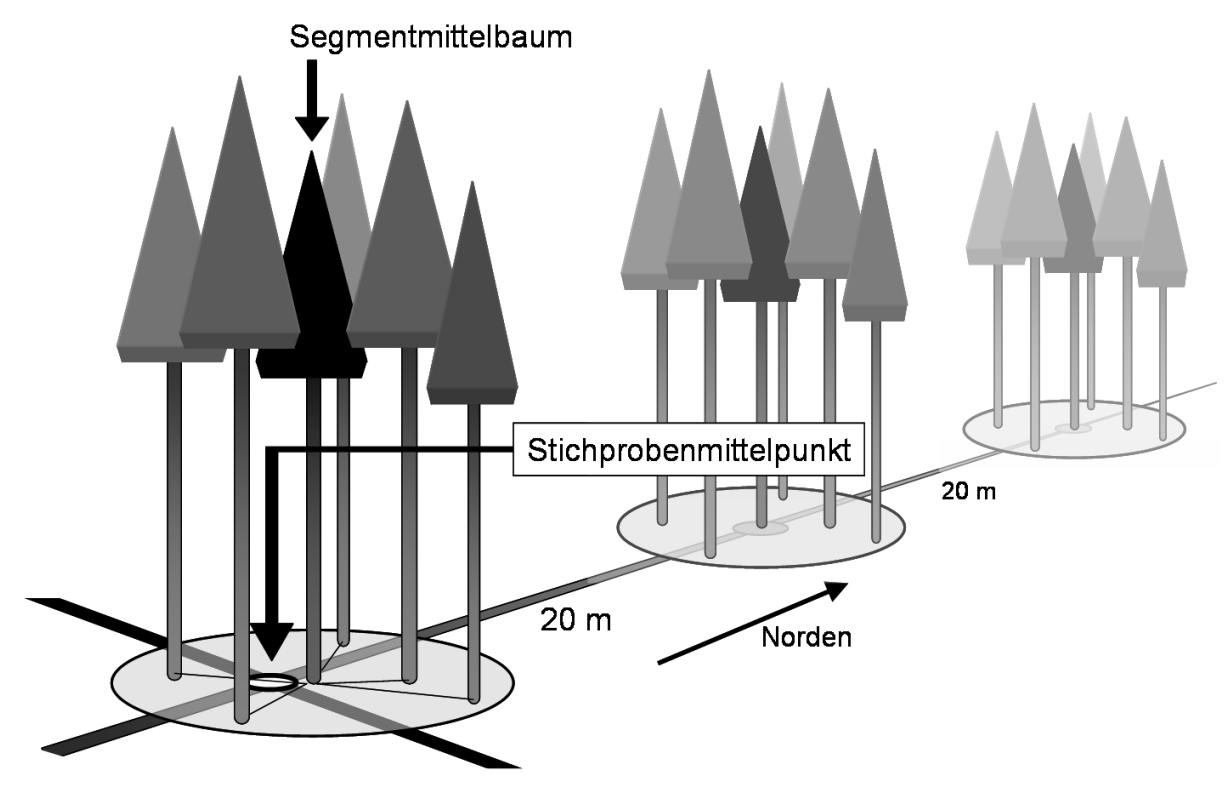

Abbildung 2.2: Inventurverfahren nach TRISL 1998. Der Schnittpunkt der Gitternetzlinien kennzeichnet den Stichprobenmittelpunkt; der nächste Baum und dessen fünf nächsten Nachbarn ergeben das erste Segment.

der zunächst ein systematisches Gitternetz mit zufällig ausgewählten Startkoordinaten über das Gebiet gelegt wird. Neben dem Zeitraum der Aufnahme (im Harz zwischen April und Juni, im Solling zwischen August und Oktober) unterschieden sich beide Gebiete in ihrer Netzweite voneinander. Im Harz lagen die Schnittpunkte der Linien 400 m auseinander, während im Solling eine Netzweite von 250 m gewählt worden war. In der Erfassung des Schälschadensaufkommens und dem weiteren Vorgehen stimmten aber beide Gebiete überein. An jedem Schnittpunkt des Gitternetzes wurde ein Stichprobenmittelpunkt etabliert, der sowohl als Startpunkt des ersten Segmentes als auch als Referenz für die Lokalisierung der zwei weiteren Segmente der Klumpenstichprobe diente. Deren Startpunkte wurden $20 \mathrm{~m}$ und $40 \mathrm{~m}$ nördlich des Stichprobenmittelpunktes definiert. Abbildung 2.2 verdeutlicht das dreiteilige Stichprobencluster. An jedem Segmentmittelpunkt wurde der nächstgelegene Baum ausgewählt und zusammen mit seinen nächsten fünf Nachbarbäumen in die Segmentgruppe aufgenommen. Mit je sechs Bäumen in den Segmenten ergab dies die Summe von 18 Bäumen je Stichprobenpunkt. An jedem dieser Bäume wurde der Stamm auf Schälschädigungen untersucht und hinsichtlich deren Alter klassifiziert. Unterschieden wurde zwischen Altschäle und Neuschäle sowie zwischen frischer Sommer- und frischer Winterschäle. 
Als frische Winterschäle galt solche, deren Verursachung zwischen November und März der vorangegangenen Winterperiode datiert wurde.

Fielen einzelne Segmente auf Flächen ohne Baumbestand, z.B. auf Wege, Blößen oder Holzlagerplätze, ergaben sich Datensätze mit lediglich sechs oder zwölf aufgenommenen Bäumen. Stichprobenpunkte mit unvollständig aufgenommenen Segmenten, also mit weniger als sechs erfassten Bäumen je kartiertem Segment, wurden im Zuge dieser Untersuchung aussortiert. $\mathrm{Zu}$ Beginn der Inventur waren zudem nur solche Schnittpunkte als Stichprobenmittelpunkt ausgewählt worden, die innerhalb vorab definierter Bestände lagen. In dieser Auswahl ging es darum, nur dort Inventurpunkte zu etablieren, wo mit einer erhöhten Schälgefährdung für die Bestände zu rechnen war. Die Kriterien bezogen sich hierbei auf die Schälfähigkeit des dort stockenden Bestandes und war abhängig von Baumart und -alter. Für Fichtenstandorte galt ein Alter von 15 bis 60 Jahren, für Buchenstandorte ein Alter von 15 bis 70 Jahren als Richtwert (TRISL 1998, TRISL ET AL. 1999).

\subsubsection{Datenumfang und Dateneignung}

Einmal eingerichtete Stichprobenpunkte wurden in der Regel in den Folgejahren erneut aufgenommen. Durch strukturelle Veränderungen im Wald entfielen jedoch auch Punkte und es kamen neue hinzu, was zu Schwankungen in der Anzahl der Stichprobenpunkte zwischen den Jahren führte. Für die vorliegende Analyse wurden darüber hinaus nur solche Datensätze ausgewählt, die in Ihrer Verknüpfung mit anderen Parametern keine Informationslücken aufwiesen. So umfasst der zugrunde liegende Datenpool insgesamt 6.319 Datensätze, von denen etwa 40 \% aus dem Harz und $60 \%$ aus dem Solling stammen (vgl. Tab. 2.2).

Abbildung 2.3 zeigt den Anteil der Baumarten Fichte und Buche am Datenpool aller Inventuren in Solling und Harz. Die Auswertung bezieht sich auf Einzelbaumwerte, also die Anzahl der erfassten und hinsichtlich ihrer Schälschädigung klassifizierten Bäume innerhalb der Stichprobenpunkte. Mit 46 \% fällt der Fichtenanteil an der Gesamtprobe im Solling deutlich geringer aus, als mit $58 \%$ im Harz. Absolut betrachtet erreichen die Zahlen im Solling jedoch höhere Werte, da der Datenpool insgesamt größer ist. Es stehen dort Datensätze von 27.507 erfassten Fichten zur Verfügung, während es im Harz 22.601 Datensätze sind. Den zweitgrößten Anteil an den Inventurdaten nimmt die Buche ein. Für diese Baumart liegen im Solling 25.748 und im Harz 12.283 Einzelbaumwerte vor. 


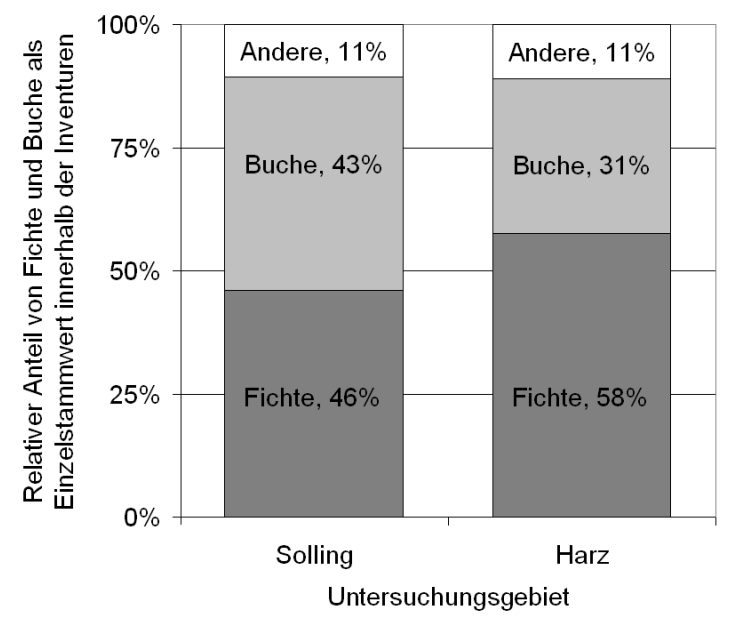

Abbildung 2.3: Anteil der Baumarten Fichte und Buche innerhalb aller Inventurpunkte in Harz und Solling; dargestellt als Relativwert

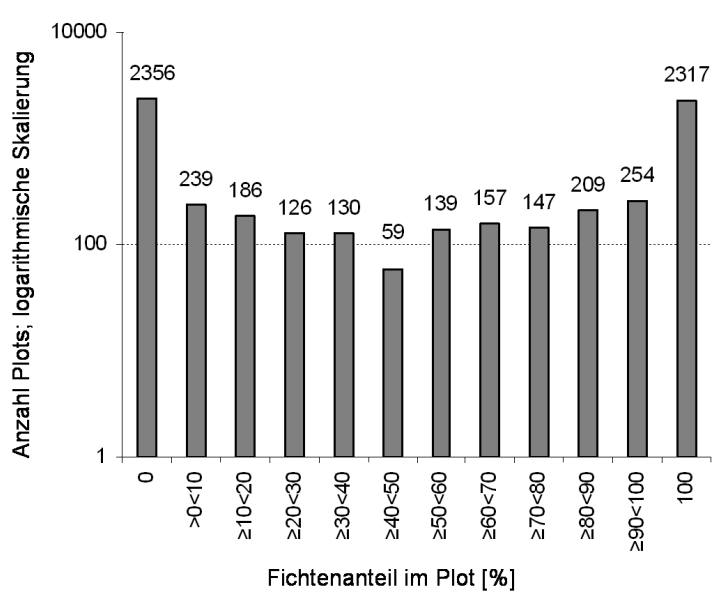

Abbildung 2.4: Fichtenanteil innerhalb der Stichprobenpunkte; klassifiziert in $10 \%$ Schritten und mit logarithmischer Skalierung abgetragen

Für die späteren Analysen wichtig zu unterscheiden ist auch der Anteil der Baumarten innerhalb der Stichprobenpunkte. Das Wissen um Stichprobenpunkte mit nur einer Baumart ist insofern von Bedeutung, als dass Modelle, in die die abhängige Variable in binär codierter Form eingehen soll, eine „Vollbesetzung“ des Stichprobenpunktes mit der betrachteten Baumart voraussetzen. Um einen Eindruck von der Häufigkeitsverteilung der Stichprobenpunkte in Bezug auf deren Fichtenanteil zu be-

Tabelle 2.2: Stichprobenumfang der Inventuren zur Schälschädigung, angegeben in Anzahl je erfasster Winterperiode; Aufnahmezeitraum im Harz: April bis Juni 2002, '04 und '06; Aufnahmezeitraum im Solling: August bis Oktober 2002, '03 und '06; Gesamtzahl: 6.319

\begin{tabular}{lrrrrr}
\hline Teilgebiet & $2001 / 02$ & $2002 / 03$ & $2003 / 04$ & $2005 / 06$ & $\sum$ \\
\hline Harz Forstamt I & 104 & - & 146 & 159 & 409 \\
Harz Forstamt II & 159 & - & 215 & 233 & 607 \\
Harz Forstamt III & 136 & - & 176 & 183 & 495 \\
Harz Forstamt IV & 151 & - & 183 & 186 & 520 \\
Harz Forstamt V & 112 & - & 177 & 188 & 477 \\
Summe Harz: & 662 & - & 897 & 949 & 2.508 \\
Solling Forstamt I & 286 & 286 & - & 335 & 907 \\
Solling Forstamt II & 488 & 490 & - & 544 & 1.522 \\
Solling Forstamt III & 454 & 454 & - & 474 & 1.382 \\
Summe Solling: & 1.228 & 1.230 & - & 1.353 & 3.811 \\
\hline
\end{tabular}


Tabelle 2.3: Stichprobenpunkte, die ausschließlich mit Fichten oder Buchen besetzt sind; angegeben in absoluter Anzahl über alle Inventurperioden hinweg; differenziert nach Baumart und Untersuchungsgebiet sowie gegliedert nach Baumzahl im Stichprobenpunkt; $n=3753$

\begin{tabular}{lrrrr}
\hline Anzahl der Segmente & III (18 Bäume) & II (12 Bäume) & I (6 Bäume) & $\sum$ \\
\hline Reine Fichtenplots Harz & 773 & 199 & 68 & 1.040 \\
Reine Fichtenplots Solling & 894 & 251 & 132 & 1.277 \\
Reine Buchenplots Harz & 250 & 90 & 60 & 400 \\
Reine Buchenplots Solling & 703 & 229 & 104 & 1.036 \\
\hline
\end{tabular}

kommen, sind in Abbildung 2.4 die relativen Anteile dieser Baumart innerhalb der Stichprobenpunkte dargestellt. Die hierfür berechneten Werte wurden in Klassen zusammengefasst, deren Grenzen in Schritten zu jeweils $10 \%$ festgelegt wurden. Stichprobenpunkte ohne Fichten und solche, die ausschließlich mit Fichten besetzt waren, wurden gesonderten Klassen zugewiesen („0“ und „100“). In der sich ergebenden zweigipfeligen Verteilung nehmen diese beiden Klassen extreme Werte an, so dass für die Gesamtdarstellung eine logarithmische Skalierung gewählt wurde. Der in der Abbildung ganz rechts wiedergegebene Wert für reine Fichtenstichprobenpunkte zeigt, dass die Beschränkung auf jene Stichprobenpunkte einen Datenumfang von 2.317 Stichprobenpunkten generieren würde. Dies entspricht einem Anteil von etwa $59 \%$ am Datenpool der Stichprobenpunkte mit Fichtenvorkommen. Auf der Ebene der einzelnen Bäume betrachtet, schließt dieser Wert aber immerhin 73 \% der erfassten Fichten ein (36.606 von 50.108).

Eine weiterführende Stratifizierung dieser $100 \%$-Klasse bietet Tabelle 2.3, in der ausschließlich Stichprobenpunkte mit nur einer erfassten Baumart aufgeführt sind und in welcher zwischen Stichprobenpunkten mit 6, 12 oder 18 Bäumen unterschieden wird. In nennenswertem Umfang gilt dies, neben der Fichte, jedoch nur noch für die Baumart Buche, deren Verwendung als abhängige Variable im Modell allerdings eine Schwierigkeit entgegen steht. Wie in 2.2.1 erläutert, wurden die Inventuren im Sommerhalbjahr durchgeführt. Aus diesem Umstand ergeben sich zwei wichtige Aspekte, die bei der Analyse berücksichtigt werden müssen und zu Restriktionen in der Verwendbarkeit der Daten führen:

- Der Zeitraum der Kartierungen weicht in den beiden Untersuchungsgebieten deutlich voneinander ab und umschließt insgesamt eine Spanne von 6 Monaten. 
- Auch innerhalb eines Gebietes konnten die Inventurmaßnahmen nicht in allen Teilgebieten simultan erfolgen, sondern unterscheiden sich in ihrem Aufnahmezeitpunkt um bis zu 10 Wochen voneinander.

In Bezug auf die Auswertung von Sommerschäle an Buche ergibt sich hieraus ein Problem. Wenn die Inventur stattfindet, während sich die betrachtete Schälaktivität noch im Prozess befindet und sich die Aufnahme über einen Zeitraum von 10 Wochen hinzieht, weisen die Stichprobenpunkte eine unterschiedliche Chance für das Vorhandensein eines aktuellen Schälschadens auf. Wollte man wiederum die gesamte Periode der Sommerschälaktivität auf den Wert 100 \% setzen und die bis zum konkreten Aufnahmezeitpunkt verstrichene Zeit als relativen Anteil hiervon betrachten, wäre auch die Gewichtung der Chance mit diesem Wert keine adäquate Lösung des Problems. In jenem Fall müsste man voraussetzen, dass die Schälaktivität des Rotwildes über die gesamte Zeit und über den gesamten Raum verteilt konstant wäre. Diese Annahme dürfte nach derzeitigem Wissensstand zum Raum-Zeit-Verhalten des Rotwildes jedoch nicht legitim sein. Bei der Erfassung der Winterschäle ergeben sich diese Hindernisse nicht, da die Periode des Schadeneintritts bereits vollständig abgeschlossen ist und der genaue Zeitpunkt der Aufnahme in diesem Fall keinen Einfluss auf das Ergebnis selbst nehmen kann. Auf eine Auswertung von Schälinventurdaten der Baumart Buche wird aus diesem Grund verzichtet.

\subsubsection{Verwendeter Datenpool}

Für die anstehenden Untersuchungen steht somit ein Datenpool von insgesamt 3.963 Stichproben zur Verfügung. Im oberen Teil der Tabelle 2.4 auf Seite 19 sind diese den jeweiligen Datensets zugeordnet. Es ergeben sich also 1.667 homogene Stichproben, wovon 773 im Harz und 894 im Solling liegen. Neben der Verteilung der Datensätze auf die Gebiete gibt die Tabelle darüber hinaus Aufschluss über die Zahl der aufgesuchten Stichprobenstandorte und die Bedingungen, die zur Aufnahme der jeweiligen Datensätze in ein Datenset geführt haben. Auf der Basis der im oberen Teil dargestellten homogenen Datensets sollte die binäre logistische Regressionsrechnung ursprünglich durchgeführt werden. In Folge dessen fußen auch die folgende Charakterisierung der Stichprobenpunkte (Kapitel 3) und die Analyse des Schälschadenaufkommens (Kapitel 4) auf diesem Datensatz. Zusätzlich ist die Charakterisierung jedoch in gleicher Weise für die als heterogen bezeichneten Stichproben getrennt vorgenommen worden, sodass hierfür der vollständige Datenpool von 3.963 Datensätzen genutzt wurde. 
Tabelle 2.4: Gesamtübersicht zum jeweils verwendeten Datenpool in den Kapiteln

\begin{tabular}{|c|c|c|c|c|}
\hline Kapitel & Datenset & Stichproben & Standorte & Bedingungen \\
\hline \multirow[t]{8}{*}{$3+4$} & Harz (homogene Plots) & 773 & & 18 Fichten \\
\hline & Harz (heterogene Plots) & 932 & & 1 Fichte \\
\hline & Summe Harz & 1.705 & & \\
\hline & Solling (homogene Plots) & 894 & & 18 Fichten \\
\hline & Solling (heterogene Plots) & 1.364 & & 1 Fichte \\
\hline & Summe Solling & 2.258 & & \\
\hline & Gesamt homogen & 1.667 & & \\
\hline & Gesamt & 3.963 & & \\
\hline \multirow[t]{3}{*}{$5+6$} & Harz & 773 & & 18 Fichten \\
\hline & Solling & 894 & & 18 Fichten \\
\hline & Gesamt & 1.667 & & 18 Fichten \\
\hline \multirow[t]{3}{*}{7} & Harz & 1.338 & 446 & 1 Fichte, 3 Jahre \\
\hline & Solling & 1.866 & 622 & 1 Fichte, 3 Jahre \\
\hline & Gesamt & 3.204 & 1.068 & 1 Fichte, 3 Jahre \\
\hline
\end{tabular}

Kapitel 3: Charakterisierung der Stichprobenpunkte, Kapitel 4: Analyse des Schälschadenaufkommens, Kapitel 5: Vorbetrachtung der Chancenverhältnisse, Kaptitel 6: Binäre logistische Regressionsmodelle, Kapitel 7: Generalisierte lineare Modelle

Für die Vorbetrachtung der Chancenverhältnisse (Kapitel 5) und die darauf basierenden binären logistischen Regressionsmodelle (Kapitel 6) werden dann nur noch die homogenen, also mit 18 Fichten besetzten Stichprobenpunkte verwendet. Für die letztendliche Analyse in verallgemeinerten linearen Modellen, welche unter Berücksichtigung der relativen Fichtenanteile im Stichprobenpunkt auch solche Stichproben zulässt, die nicht vollständig mit 18 Fichten besetzt sind, steht dann wieder ein deutlich größerer Datenpool zur Verfügung. Mit der Bedingung einer wiederholten Aufnahme des Stichprobenpunktes in allen drei Inventurjahren und dem Vorkommen mindestens einer Fichte im Stichprobenpunkt lassen sich nun insgesamt 3.204 Datensätze für die Analyse verwenden. Im Harz werdem damit 1.338 und im Solling 1.866 Stichproben untersucht, deren Beobachtungen auf insgesamt 1.068 Einzelstandorten beruhen. 


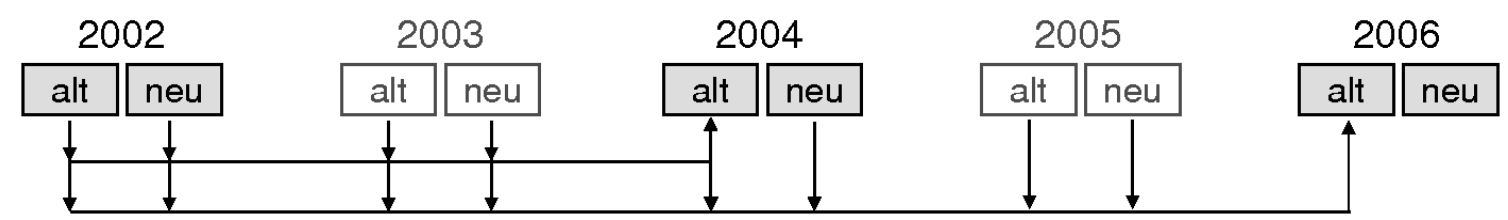

Abbildung 2.5: Schematische Darstellung des Informationsflusses zur Altschäle am Beispiel Harz; im Jahr 2002 enthält „alt" alle noch registrierbaren Schälschaden der Vergangenheit; die Inventuren fanden in den Jahren 2002, 2004 und 2006 statt

\subsection{Herkunft der unabhängigen Variablen}

\subsubsection{Quantifizierung vorangegangener Schälschäden}

Bei der Information über das Vorhandensein alter Schälschäden ist eine aufmerksame Betrachtung erforderlich. Zwar lassen sich alte Schäden per Definition von neuen, also frischen Schäden abgrenzen (vgl. 2.2.1. Inventurverfahren, S. 13), doch ist eine genaue Datierung der alten Schäden auf der Grundlage der Inventurdaten nicht möglich. Abbildung 2.5 verdeutlicht hierfür zunächst den Informationsgehalt des Parameters Altschäle und zeigt dessen Bündelung im Datenpool am Beispiel der Inventurjahre des Untersuchungsgebietes Harz. Demnach umfasst der Wert je nach Inventurjahr die folgenden Informationen:

- 2002: Sämtliche Schälschadensereignisse der Vergangenheit, die sich noch eindeutig als solche ansprechen lassen und nicht aus der Winterperiode 2001/02 stammen.

- 2004: Alle im Jahr 2002 als alt eingestuften Schälschadensvorkommen sowie hinzugekommene Schälschäden des Zeitraumes ab der letzten Inventur im Sommer 2002 bis zum Beginn der Winterschälaktivität im Herbst 2003.

- 2006: Entsprechend die im Jahr 2004 dargestellten Altschälschadenssummen zuzüglich der Schälschäden, die sich ab der Inventur 2004 bis zum Herbst 2005 ereignet haben.

An dieser Auflistung erkennt man, dass die gesonderte Darstellung alter und neuer Schälereignisse für die Jahre 2003 und 2005 in Abbildung 2.5 rein theoretischer Natur ist. Eine Differenzierung dieser beiden Klassen anhand der Aufnahmedaten ist nicht anwendbar. Für die Bewertung der kontinuierlichen Schälaktivität an einem Stichprobenpunkt ergeben sich hieraus einige Einschränkungen. So ist es beispielsweise nicht 
möglich, auf den Altschälschadensanteil des Jahres 2003 zu schließen, indem man die Summe aus alten und neuen Schäden des Vorjahres (2002) vom Altschälwert des nachfolgenden Jahres (2004) abzieht. Alte und frische Schälschäden lassen sich in diesem Fall ja nicht voneinander trennen. Zudem wird ein Schälschaden des Jahres 2003 auf oder neben einem Schaden des Jahres 2001 zum Aufnahmezeitpunkt der Inventur 2004 gleichermaßen als Altschaden klassifiziert wie der noch ältere Schaden am selben Baum. An der Anzahl altgeschälter Bäume im Stichprobenpunkt ändert sich also nichts, obwohl in der Zwischenzeit ein erneutes Schälereignis eingetreten ist.

Um nun dennoch ein Maß für die Kontinuität der Schälschädigung eines Stichprobenpunktes generieren zu können, muss auf die klar umgrenzten Werte der Neuschälereignisse der Inventurjahre zurückgegriffen werden. Nur hierdurch lassen sich Mehrfachwertungen desselben Altschälschadens vermeiden. Zur Klassifizierung sind somit nur solche Stichprobenpunkte geeignet, an denen in allen drei aufeinander folgenden Inventurjahren Daten erhoben wurden. Diese Bedingung trifft im Harz auf 446 und im Solling auf 627 Punkte zu. Um zumindest diesen Stichprobenumfang beizubehalten, beziehen sich die Datensets auf alle Stichprobenpunkte mit mindestens einer Fichte, also sowohl auf heterogen wie auch homogen besetzte Plots gleichermaßen. Der Wert dient somit einer Auswertung nach relativem Schälprozent und steht nicht für Rechenmodelle zur Verfügung, in die die abhängige Variable in binärer Form eingeht. Als Bezugswerte kommen in diesem Fall zudem ausschließlich die Inventurdaten des Jahres 2006 in Frage.

Die Beschränkung auf eine einfache Codierung ergibt drei Klassen: 0-1-2. Erfolgte in keinem der beiden vorangegangenen Inventurjahren eine Neuschälkartierung im Stichprobenpunkt, bekommt dieser den Wert 0. Wurde nur in einem Jahr Neuschäle festgestellt, fällt der Punkt in die Klasse 1. Bei zweimaligem Vorkommen von Neuschälschäden am Punkt wird dieser entsprechend mit 2 codiert. Bei der späteren Auswertung der mittleren Neuschälprozente des Jahres 2006 werden die Ergebnisse dieser drei Klassen dann untereinander verglichen.

\subsubsection{Geländespezifische Merkmale}

Digitale Geländemodelle, realisierbar durch Geographische Informationssystemẹ bieten nicht nur hilfreiche Darstellungen, sondern auch vielfältige Möglichkeiten der Da-

\footnotetext{
${ }^{1}$ Abkürzung = GIS; In diesem Fall unter Verwendung von Arc View 9.0 von ESRI Geoinformatik GmbH, Kranzberg.
} 
tengewinnung. Für forstliche Fragestellungen interessant ist meist die Charakterisierung des Standortes. Die für die vorliegenden Modellrechnungen zur Verfügung stehenden Parameter „Höhe“, „Hangneigung“, „Exposition“, „Exponiertheit“ und „Einstrahlungspotential“" wurden gewählt, um die geländemorphologischen Gegebenheiten berücksichtigen zu können ${ }^{2}$. Mit der absoluten Höhe über Normalnull liegt die wohl einfachste Information über die Lage der Stichprobenpunkte im Raum vor. Nach MuES (2000) stellt die Höhe über NN im Niedersächsischen Bergland jedoch die wichtigste Einflussgröße für die Niederschlagsmenge dar, welche im Rahmen dieser Untersuchung nicht erhoben werden konnte. Stellvertretend für Phänomene dieser Art (neben der Niederschlagsmenge z.B. Temperatur, Schneehöhe und Schneeliegedauer) wird die Höhe also als Parameter in die Modelle eingehen, von dem vor allem indirekte Einflüsse auf die räumliche Verteilung von Schälschäden erwartet werden.

Die Hangneigung der Stichprobenpunkte wurde zunächst in Form der Steigung der direkt umliegenden Fläche generiert. Hierzu wird im GIS wie folgt verfahren: Es werden neun Zellen mit jeweils 10 x 10 m Kantenlänge definiert, für deren mittlere Zelle schließlich die vermutliche Steigung in Annäherung hergeleitet werden soll. Innerhalb der 30 x 30 m großen Berechnungsfläche wird der Steigungsgradient in zwei Richtungen jeweils separat kalkuliert (Nord-Süd-Richtung und West-Ost-Richtung). Mit Kenntnis der Einzelgradienten ergibt sich dann die Steigung am Punkt als Verhaltniswert beider Gradienten (vgl. z.B. Burrough And MCDonnel 1998) und wird üblicherweise in Prozent angegeben ${ }^{3}$. Eine Steigung von $17 \%$ bedeutet demnach eine Höhenzunahme von 17 Metern $(\Delta y)$ auf 100 Metern horizontaler Distanz $(\Delta x)$. Die Verwendung von Steigungsprozentwerten birgt jedoch die Gefahr der Missinterpretation von Verhältnissen. So entspricht eine Steigung von $100 \%$ einem Neigungswinkel von $45^{\circ}$. Der Neigungswinkel einer $50 \%$ igen Steigung entspricht jedoch nicht $22,5^{\circ}$ (also der Hälfte von $45^{\circ}$ ), sondern 26,6 . Aus diesem Grund wurden die Steigungsprozentwerte für die vorliegende Untersuchung zusätzlich über den Arcustangens zunächst in RAD umgerechnet und dann in Grad überführt (Gl. 2.6). Zwar sollen bei der Einteilung in hangabhängige Expositionsklassen weiterhin Steigungsprozente verwendet werden, doch geschieht dies nur zwecks Orientierung an vordefinierten Klassengrenzen. In die Modellrechnungen gehen dann sämtliche Hangneigungswerte in Grad ein.

\footnotetext{
${ }^{2}$ Alle Berechnungen erfolgten durch Dr. R. Schulz, Abteilung Ökoinformatik, Biometrie und Waldwachstum des Büsgeninstituts der Georg-August-Universität Göttingen.

${ }^{3}$ Der Wert aus Gleichung 2.5 wird hierzu also mit 100 multipliziert.
} 
Tabelle 2.5: Grenzwerte der Expositionsklassen; $N=$ Nord, $N O=$ Nordost, $O=$ Ost, $S O=$ Südost, $S=$ Süd, $S W=$ Südwest, $W=$ West, $N W=$ Nordwest; Punkte mit Hangneigung $=0$ gelten als Plateau"

\begin{tabular}{lrrrrrrrr}
\hline Klasse & $N$ & $N O$ & $O$ & $S O$ & $S$ & $S W$ & $W$ & $N W$ \\
\hline Grad & $339-23$ & $24-68$ & $69-113$ & $114-158$ & $159-203$ & $204-248$ & $249-293$ & $294-338$ \\
\hline
\end{tabular}

Als Exposition wird in dieser Arbeit die Neigungsrichtung bezeichnet. Die Werteskala reicht von $>0^{\circ}$ bis $360^{\circ}$ azimuth. Entsprechend dem Start in Nordrichtung weist $90^{\circ}$ nach Osten, $180^{\circ}$ nach Süden und $270^{\circ}$ nach Westen. Stichprobenpunkte mit einem Hangneigungswert von „0“ erhielten bei der Berechnung den Wert -1. Mit dem Expositionswert erhält jeder Datensatz eine weitere Information über die geographischen Verhältnisse am Stichprobenpunkt. Gerade bei dieser Art von Parametern ist jedoch zu berücksichtigen, dass hierdurch bedingte Effekte auf die Zielvariable von mehreren Faktoren abhängig sind. Über die Codierung der Himmelsrichtungen gibt Tabelle 2.5 Auskunft, in welcher die Grenzwerte der Expositionsklassen in Grad angegeben sind.

Die relative Exponiertheit in der weiteren Umgebung ist ein Index, der von ScHulZ (2003) als ein Maß zur Beurteilung der Lage eines Punktes im Raum verwendet wurde. Die Exponiertheit eines Punktes ist hierbei abhängig von Geländeerhebungen der Umgebung. Sowohl die Höhendifferenz des Punktes zu Erhebungen der Umgebung als auch die Distanz zwischen beiden bestimmen den Exponiertheitswert. Grundlage der Berechnung ist wiederum ein digitales Geländemodell, welches für jeden beliebigen Punkt einen zugehörigen Wert ermitteln lässt, welcher im Folgenden durch „exp2“ abgekürzt wird ${ }^{4}$.

Der Wert exp2 wird dabei aus mehreren raumbezogenen Werten modelliert. Im Geländemodell wird jede Fläche mit einem fiktiven Lichtstrahl „beleuchtet“. Insgesamt kommen hierfür 36 Richtungen $\left(0^{\circ}, 10^{\circ}, 20^{\circ}\right.$ bis $350^{\circ}$ azimuth) zur Anwendung. Der Winkel der fiktiven Einstrahlung beträgt hierbei $2^{\circ}$. Das Höhen- und Distanzverhältnis zu den umliegenden Geländeformationen bestimmt in diesem Prozess für jede neue Strahlungssituation einen Wert, der 0 oder 1 (beleuchtet nein/ja) annehmen kann. Für exp2 ergibt sich aus der Addition der Einzelwerte schließlich der Endwert. Entsprechend der 36 Richtungen liegt dieser zwischen 0 und 36 (Schulz 2003). In

\footnotetext{
${ }^{4}$ Die 2 steht für den Einstrahlungswinkel von $2^{\circ}$, der im Weiteren erläutert wird. Es existieren weitere Indizes dieser Art, berechnet in anderen Variationen und anderen Winkeln, auf die hier aber nicht weiter eingegangen werden soll.
} 
der Analyse der Stichprobenstandorte werden jedoch 37 Klassen ausgewiesen, da der Wert 0 als zusätzliche Klasse ausgeschieden wird.

Bei dem Parameter der potentiellen Einstrahlung handelt es sich um einen theoretischen Wert, der als Index ähnliche Aussagen über den geographischen Charakter eines Standortes liefern soll, wie der Grad der Exponiertheit. Tatsächlich liegen diesem Wert die Berechnungen der Exponiertheit als Vorüberlegungen zugrunde. So drückt das Einstrahlungspotential aus, mit welchem Strahlungsgenuss an einem bestimmten Tag des Jahres an einem Punkt zu rechnen ist. In der vorliegenden Untersuchung wurde hierfür der 15. Januar gewählt. Da es hierbei lediglich um Vergleichswerte zwischen den Punkten geht, steht der 15. Januar stellvertretend für die Winterperiode als ein Beispieltag zur Verfügung. Grundlage der Berechnung sind also die Sonnenstände, im Sinne ihres Strahlungswinkels zu den Stichprobenpunkten, und die sich hieraus und aus der Geländemorphologie der Umgebung ergebenden Zeiteinheiten der Bestrahlung eines Punktes. Der maximale Wert, den ein Standort hierbei erreichen kann, sind 9 Stundeneinheiten. Entsprechend reicht die Intervallskala mit zehn Klassen von 0 (wenn der Punkt am 15. Januar zu keiner Zeit des Tages direkter Sonnenstrahlung ausgesetzt ist) bis 9 .

Um einen theorethischen Wert handelt es sich, weil dieser Parameter die Vegetation vor Ort nicht berücksichtigt. Es liegen also keine Messwerte vor, deren absoluten Werte Aufschluss über tatsächlich vorhandene Strahlung am Boden geben könnten. Aufgrund dieser Unsicherheiten und des Mangels an Information soll der Parameter stattdessen als weiterer charakterisierender Faktor des Standortes genutzt und, in Anbetracht der geschilderten Einschränkungen, hierbei als Index geführt werden.

\subsubsection{Bestandesspezifische Merkmale}

Die geographische Lage eines Inventurpunktes lässt sich über die Angabe seiner GaussKrüger-Koordinaten bestimmen. Um nun Informationen über die zugehörigen Forsteinrichtungsdaten zu erhalten, können die Lageparameter der Punkte im GIS mit den Polygoneinheiten der digitalen Karte verschnitten werden, sodass eine Zuordnung der im Forsteinrichtungswerk hinterlegten Bestandesdaten an die aufgenommenen Punkte erfolgen kann.

Das Potential der Lokalisation bei einer großen Anzahl von Datensätzen, die eine automatisierte Zuweisung erfordern, endet allerdings mit der Zuordnung auf Ebene der forstlichen Unterfläche. Einzelne Bestände, von z.B. gleichaltrigen Bäumen einer 
Art, die innerhalb der Unterfläche eine weitere Differenzierung zuließen, sind hierdurch nicht eindeutig zu bestimmen. Bei der Berechnung der folgenden Parameter ist die Betrachtung der Unterfläche als Bezugsebene also stets ein Kompromiss bezüglich der Abbildungsschärfe einer Punktumgebung.

Mit Berechnung der Größe von Flächen rund um die Inventurpunkte soll in Annäherung eine Strukturbeschreibung erfolgen, welche die weitere und nähere Umgebung der Punkte bestenfalls als Differenzierungsmerkmal innerhalb der Modelle zur Verfügung stellt. Wie bereits erläutert, kann hierbei nur auf Mittelwerte zurückgegriffen werden, die sich auf den Ebenen der forstlichen Planungseinheiten berechnen lassen. Für die nähere Umgebung bietet sich die Unterfläche hierbei als kleinste Einheit an. Zur Beschreibung der weiteren Umgebung werden hingegen die Grenzen der Abteilungen verwendet.

Von weiterer Umgebung soll in diesem Zusammenhang gesprochen werden, wenn die Abteilung (vgl. 2.1.3, S. 13) eines Stichprobenpunktes als Ebene der Betrachtung dient. Unterschieden werden muss in diesem Zusammenhang nicht nur zwischen den beiden Untersuchungsgebieten Harz und Solling, sondern auch, welcher Datenpool für die Analyse verwendet werden soll. Im Fall der binären logistischen Regression bliebe der Datenumfang auf solche Stichprobenpunkte beschränkt, die nur mit 18 erfassten Fichten als vollbesetzt gelten können $(\mathrm{n}=1.667$ Plots). Bei Modellen zur Fichtenschäle, in die die abhängige Variable nicht in binärer Form eingeht, erhöht sich dagegen die Anzahl der verwendbaren Plots $(\mathrm{n}=3.963)$. Und auch deren innere und äußere Baumartenausstattung 5 verändern sich. Ihre Relevanz zeigt diese Überlegung allerdings nicht bei der Auswahl der Datensätze für die verschiedenen Modelle, denn während dieser Prozedur befindet sich die Information ja ohnehin schon am jeweiligen Wert des Stichprobenpunktes, sondern vielmehr bei der anschließenden Interpretation der Ergebnisse. Denn die Voraussetzungen für die Aussagefähigkeit der beobachteten Effekte sind in beiden betrachteten Szenarien eben nicht die selben.

Legt man also zunächst nur Stichprobenpunkte mit drei vollständigen Segmenten und einem Fichtenanteil von $100 \%$ zugrunde, sind rund $97 \%$ der 1.667 Plots dem Bestandestyp (BT) 50 zugeordnet ${ }^{6}$. Die ins Modell eingehenden Punkte befinden sich dann also fast ausschließlich innerhalb von Fichtenreinbeständen. Zwar bietet der Be-

\footnotetext{
${ }^{5}$ Mit innerer Baumartenausstattung ist die Artenzusammensetzung der im Stichprobenpunkt erfassten Bäume gemeint. Die äußere Baumartenausstattung bezieht sich auf die Bäume der Umgebung.

${ }^{6}$ Übersichten zur Codierung der Bestandestypen und Baumarten befinden sich im Anhang unter den Abschnitten A und B.
} 
standestyp keine direkte Information über die vor Ort tatsächlich stehenden Bäume, doch lässt die wirtschaftliche Ausrichtung des Bestandes erste Einteilungen und Erwartungen zu, in welchem Umfeld sich die Stichprobenpunkte befinden.

Die nähere Umgebung soll die weitere Umgebung als etwas konkretere Betrachtungsebene ergänzen. Als Maßstab werden hier die umliegende die Unterfläche oder ggf. die Unterabteilung herangezogen, um die Gegebenheiten um den Stichprobenpunkt herum stärker einzugrenzen. Im Sinne einer möglichst gleichartigen Skalierung für alle Untersuchungspunkte muss hierbei (wie im Übrigen auch bei der Wahl der anderen Ebenen) ein Kompromiss eingegangen werden. Weder Flächengröße, noch Form, noch Umfang der Unterflächen und -abteilungen der verschiedenen Stichprobenpunkte sind gleich. Eine Abkehr von den bestehenden Grenzen des Forsteinrichtungswerkes wäre jedoch nicht pragmatisch und aufgrund vielfach höherer Unsicherheiten bei der Werteberechnung wohl auch nicht zielführend. Als eine Möglichkeit der Normierung bietet sich die Verrechnung mit der Fläche als relativem Bezugspunkt an. Für die Baumartendiversität ergibt sich dann die Angabe „Baumartenzahl je 10 ha Abteilungsfläche“.

Aufgrund der Zweckgebundenheit der Inventur zur Schälschadenskartierung fand bereits durch die Aufnahme der Stichprobenpunkte eine Auswahl hinsichtlich des Alters der Bestände statt (vgl. 2.2.1, S. 13). Die durch die Inventur erfassten Altersklassen befinden sich also entsprechend der Zielvorgabe in einem Bereich potentiell hohen Schälrisikos. Sowohl das mittlere Bestandesalter $\left(r^{2}=0,871\right)$ als auch die mittlere Bestandesoberhöhe $\left(r^{2}=0,983\right)$ sind mit dem Brusthöhendurchmesser korreliert.

\subsubsection{Jagdstreckendaten als Parameter}

Die Bejagung des Rotwildes unterliegt in Deutschland gesetzlich festgelegten Bestimmungen. Die Vorgabe der zu erlegenden Anzahl Rotwildes durch detaillierte Abschusspläne bedingt den schriftlich geführten Nachweis der getätigten Abschüsse. Die über die beteiligten Forstreviere erfassten Streckenmeldungen werden in einer zentralen Datenbank (ORACLE) der Niedersächsischen Landesforsten geführt, welche alle Erlegungen sowie sämtliches registriertes Fallwild des relevanten Zeitraumes enthält. Die Streckenmeldungen weisen neben Geschlecht und Altersklasse des erlegten Tieres auch das Datum und den Ort der Erlegung aus. Letzterer liegt auf Abteilungsebene vor (vgl. 2.1.3 auf Seite 13).

Die am 01. Januar 2005 innerhalb der Niedersächsischen Landesforsten in Kraft getretene Verwaltungsreform ergab vielerorts Änderungen der Flächenzugehörigkeit über 
Revierförstereigrenzen und Forstamtsgrenzen hinweg. Hierbei wurden, wo erforderlich, insbesondere auch die Abteilungsnummern angeglichen, um eindeutige Identifikationen innerhalb eines Forstamtes zu erhalten. In der vorliegenden Arbeit bedingte dies die Überprüfung sämtlicher Abteilungen, in denen vor dem 01.01.2005 Rotwilderlegungen stattgefunden hatten. Bei verändert vorgefundenen Nummerierungen wurde die alte Klassifizierung dann durch die neue ersetzt. Hinsichtlich ihrer Anrechnung auf bestimmte Einheiten ergab sich also vielfach eine Neuzuweisung der Streckenmeldungen. Die Daten der Periode bis 01.01.2005 wurden also rückwirkend auf die Buchungseinheit der nachfolgenden Periode gesetzt. Grundsätzlich wäre dieses Vorgehen in gleicher Weise für die Aktualisierung der Abteilungszugehörigkeiten im Zuge der zuvor am 01.10.2001 in Kraft getretenen Reform denkbar gewesen. Die für diesen Zeitraum mit deutlichen Unsicherheiten behaftete Informationslage sowie der hohe Aufwand für die nur wenige Wochen umfassende relevante Periode der Abschussmeldungen, ließen dieses Vorhaben jedoch als nicht durchführbar erscheinen. Der Datenpool der Rotwildstrecke beginnt somit mit den Erlegungen und Fallwildmeldungen ab dem 01. Oktober 2001.

Um die Information über erlegtes Rotwild als Variable in die Modelle einbinden zu können, ist es zwar zielführend, Erlegungsdaten auf größerer Fläche zusammenzufassen und sie den darin liegenden Inventurpunkten als gemeinsamen Wert zuzuweisen, doch bietet sich hierfür die nächst kleinere Betrachtungsebene nach den Teilgebieten an. Als Berechnungsgrundlage sollen deshalb die Grenzen der Revierförstereien herangezogen werden, wobei die Revierflächen in Einheiten zu 100 Hektar als Bezugsgröße dienen. Die in den festgesetzten Hauptbejagungszeiträumen (vgl. 3.3.1) angefallenen Erlegungen werden somit auf Ebene der Reviere summiert, durch die jeweilige Revierfläche geteilt und mit 100 multipliziert. Auf den auf diese Weise berechneten Parameter wird Bezug genommen, wenn im Folgenden vom „Rotwildindex“ gesprochen wird.

\subsubsection{Klassifizierung von Wanderwegen}

Um ein Maß für mögliche Einflüsse durch Waldbesucher zu erhalten, wurde auf Erhebungen des Niedersächsischen Forstplanungsamtes zurückgegriffen. Dort waren Wanderwege und Ausflugspunkte des Harzes hinsichtlich ihrer Besucherfrequenz evaluiert und in kaum, mäßig und stark durch Waldbesucher genutzte Erholungszonen eingeteilt worden. Die Verschneidung mit den Probepunkten erfolgte im GIS. Da diese Bewertung nur für das Gebiet des Harzes durchgeführt wurde, liegen für den Solling keine vergleichbaren Werte vor. Der Datenumfang beschränkt sich also auf 1.552 Stichpro- 
benpunkte, von denen 315 den Wert 2 und 250 den Wert 1 tragen. Die restlichen 987 Punkte im Harz sind mit dem Wert 0 gekennzeichnet.

\subsection{Instrumente der statistischen Analyse}

\subsubsection{Statistische Tests und weitere Formeln}

Die Differenz der Mittelwerte wurde bei normalverteilten Daten mittels t-Test für zwei unabhängige Stichproben getestet und zuvor eine Varianzanalyse durchgeführt.

$\triangleright$ Variationskoeffizient

$$
V=\frac{s}{\bar{x}} \quad \text { alle } \quad \bar{x}>0
$$

$\triangleright$ relativer Variationskoeffizient

$$
V_{r}[\%]=\frac{s / \bar{x}}{\sqrt{n}} \cdot 100 \quad \text { alle } \quad \bar{x}>0
$$

$\triangleright$ Maßkorrelationskoeffizient nach Pearson

$$
r=\frac{\sum\left(x_{1}-\bar{x}\right)\left(y_{i}-\bar{y}\right)}{\sqrt{\sum\left(x_{1}-\bar{x}\right)^{2} \cdot \sum\left(y_{i}-\bar{y}\right)^{2}}}
$$

worin

$x_{i}=$ Messwert des Merkmals $X_{1}$ am $i$-ten Individuum

$y_{i}=$ Messwert des Merkmals $X_{2}$ am $i$-ten Individuum

$\bar{x} / \bar{y}=$ arithmetisches Mittel von $X_{1}$ bzw. $X_{2}$

$n=$ Anzahl aller Wertepaare

$i=$ Laufindex von 1 bis $n$

$\triangleright$ Bestimmtheitsmaß 


$$
B=r^{2}=\frac{\left(\sum x_{i} y_{i}-\frac{\left(\sum x_{i}\right) \cdot\left(\sum y_{i}\right)}{n}\right)^{2}}{\left(\sum x_{i}^{2}-\frac{\left(\sum x_{i}\right)^{2}}{n}\right) \cdot\left(\sum y_{i}^{2}-\frac{\left(\sum y_{i}\right)^{2}}{n}\right)}
$$

worin

$$
\begin{aligned}
x_{i} & =\text { Messwert des Merkmals } X_{1} \text { am } i \text {-ten Individuum } \\
y_{i} & =\text { Messwert des Merkmals } X_{2} \text { am } i \text {-ten Individuum } \\
n & =\text { Anzahl aller Wertepaare } \\
i & =\text { Laufindex von } 1 \text { bis } n \\
r & =\text { Maßkorrelationskoeffizient }
\end{aligned}
$$

$\triangleright$ Steigung

$$
m=\frac{y_{2}-y_{1}}{x_{2}-x_{1}}=\frac{\Delta y}{\Delta x}
$$

$\triangleright$ Umrechnung Steigungsprozent in Grad Neigungswinkel

$$
\text { Neigungswinkel }=\frac{\arctan \left(\frac{m}{100}\right) \cdot 180}{\pi}
$$

worin

$$
\begin{aligned}
m & =\text { Steigung in Prozent } \\
\text { arctan } & =\text { Arcustangens }
\end{aligned}
$$

\subsubsection{Logistisches Regressionsmodell}

Zur Analyse der Daten wird die Methode der binär logistischen, schrittweise rückwärts gerichteten Regression angewandt. Die abhängige Variable ist dichotom codiert $(0,1)$, wobei 1 für das Eintreten eines Neuschälschadens steht. Die Schätzung der Modellparameter (Gl. 2.7) erfolgt mittels Maximum-Likelihood-Methode, bei der jene Koeffizienten gewählt werden, welche die Wahrscheinlichkeit einer korrekten Klassifizierung der Stichprobenpunkte im logistischen Modell maximieren. 


$$
p=\frac{1}{1+e^{-}\left(\beta_{0}+\beta_{1} x_{1}+\beta_{2} x_{2}+\ldots+\beta_{j} x_{j}+a\right)}
$$

worin

$p=$ Wahrscheinlichkeit der Neuschäle (bei $p<0.05$ wird kein neuer

Schaden angenommen; Spanne: 0-1)

$e=$ Basis des natürlichen Logarithmus

$x_{1}-x_{i}=$ erklärende Variablen

$ß_{0}=$ Konstante

$ß_{1}-\bigcap_{i}=$ vom Modell geschätzte Koeffizienten für die $i$-ten erklärenden Variablen

Die Auswahl der Prädiktoren wird vom jeweils errechneten Wert des Wald-Test (Gl.2.8) abhängig gemacht (RUdOLF UND MüLlER 2004). Hiernach werden schrittweise solche Variablen ausgesondert, deren Entnahme zu keiner signifikanten Veränderung der Schadenswahrscheinlichkeit führt und die somit keinen signifikanten Beitrag zur Optimierung des Modells liefert. Von den übrigen Einflussfaktoren verbleiben im letzten Schritt der Anpassung nur jene im Modell, die mit $P<0.05$ signifikant sind.

$$
w=\left(\frac{\beta}{s_{\beta}}\right)^{2}
$$

worin

$$
\begin{aligned}
w & =\text { Wald-Wert } \\
\beta & =\text { Modellkoeffizienten } \\
s_{\beta} & =\text { Standardfehler von } \beta
\end{aligned}
$$

Auf Basis der Regressionskoeffizienten $ß$ werden die odds (Gl. 2.9) der im Modell verwendeten Prädiktoren berechnet. Die Ergebnisse lassen Aussagen darüber zu, um welchen Faktor sich die Wahrscheinlichkeit eines Schälschadeneintritts im Probepunkt 
erhöht, wenn der Wert der erklärenden Variable um eine Einheit zunimmt. Bei ordinal skalierten Prädiktoren wird als Referenz stets die erste Klasse gewählt.

$$
O R_{i}=e^{ß_{i}\left(x_{i 2}-x_{i 1}\right)}
$$

worin

$$
\begin{aligned}
O R & =\text { Chancenverhältnis für die } i \text {-te Variable } \\
\beta_{i} & =\text { geschätzter Koeffizient der } i \text {-ten Variable } \\
x_{i 2}-x_{i 1} & =\text { Veränderung der } i \text {-ten Variable bei Erhöhung um eine Einheit }
\end{aligned}
$$

Die Güte der Modellanpassung wird über den Chi-Quadrat-Test von Hosmer UND Lemeshow (2000) bewertet. Pseudo- $\mathrm{R}^{2}$-Werte werden auf Basis von Nagelkerkes $\mathrm{R}^{2}$ (RUdolF Und MÜLLER 2004) berechnet, wobei in Anlehnung an Litz (2000) pseudo$\mathrm{R}^{2}$-Werte von $>0.2$ als gut bewertet werden. Anhand einer Klassifizierungstabelle kann die Anzahl korrekt zugeordneter Fälle wiedergegeben werden.

\subsubsection{Berechnung der Chancenverhältnisse}

Die Ergebnistabellen der binären logistischen Regressionsmodelle bieten den Regressionskoeffizienten $ß$ und das so genannte odds ratio, um den Effekt der einzelnen Variablen im Modell zu bewerten (vgl. 2.4.2). Um die Aussage dieser Werte im recht komplexen Regressionsmodell nachvollziehen zu können, soll deren Entstehung im Folgenden aufgezeigt werden. Das hierzu verwendete fiktive Beispiel soll darüber hinaus verdeutlichen, wie die einzelnen Werte der Variablen in Kapitel 5 auf Seite 73 manuell in MS Excel hergeleitet wurden.

Zunächst einmal gilt die Betrachtung den Summen der geschälten und ungeschälten Beobachtungen im Gesamtdatenpool. Im binären logistischen Regressionsmodell werden diese Einheiten aus den erfassten Stichprobenpunkten generiert, welche den Wert 1 (geschält) oder 0 (ungeschält) annehmen können. In den in Kapitel 5 vorab durchgeführten Einzelanalysen der zu testenden Parameter bestehen diese Beobachtungen oder Einheiten hingegen aus den Ausprägungen der jeweiligen Fichten eines Stichprobenpunktes. Dort wird also die Information geschält/ungeschält am Baum selbst und damit in absoluter und tatsächlicher Anzahl berücksichtigt. 
Entsprechend sei nun folgendes Szenario angenommen: Der Datenpool beinhalte 2.000 Beobachtungen. Hiervon seien 400 als geschält protokolliert worden und 1.600 als ungeschält. Die Wahrscheinlichkeit einer Einheit, als geschält klassifiziert zu sein, beträgt damit im Datenpool 20 \% (400/2.000). Die Chance einer beliebigen Beobachtung, geschält zu sein, ist demnach 0,25 mal so groß (also viermal geringer), wie die, nicht geschält zu sein (400/1.600). Im Modell wird für diese Chance der englische Begriff odds verwendet. Logarithmiert man diesen Wert von 0,25 zur natürlichen Basis $e(2,71828)$, erhält man den Regressionskoeffizienten $\beta=-1,386$ für das Gesamtmodell. Beide, einander entsprechenden, Werte werden dann bei allen folgenden Berechnungen als Referenz verwendet. Im Modell werden sie als Konstante ausgegeben.

Angenommen, die 2.000 Beobachtungen verteilten sich im Gelände auf zwei verschiedene Höhenklassen und jeweils zur Hälfte lägen die Einheiten unter und über 500 Metern Höhe. Unter 500 Metern würden 100 Einheiten als geschält und 900 als ungeschält erfasst werden. Über 500 Metern seien es 300 geschälte und 700 ungeschälte. Die odds der beiden Datengruppen liegen dann bei 0,11 (10\% zu $90 \%$ ) unterhalb und bei $0,42(30 \%$ zu $70 \%)$ oberhalb der Klassengrenze von 500 Metern.

An dieser Stelle könnte man zunächst das Wahrscheinlichkeitsverhältnis zwischen diesen beiden Klassen bilden. Mit einem Verhältnis von 0,43 zu 0,11 ist die Wahrscheinlichkeit, dass eine beliebige Einheit als geschält klassifiziert ist, in der oberen Höhenklasse faktisch 3,9 mal höher als in der unteren Höhenklasse. Diesen Wert umschreibt das odds ratio.

Was im Modell ausgegeben wird und gleichsam Grundlage der Einzelbetrachtungen ist, ist jedoch das Verhältnis der jeweiligen Klassen zum Referenzwert. Im vorliegenden Beispiel werden die odds beider Höhenklassen also jeweils in Relation zum Gesamtwert von 0,25 gesetzt. Es ergeben sich odds ratios von 1,71 (hohe Lagen) und 0,44 (tiefe Lagen). Somit wird das Schälrisiko (die Chance) innerhalb der oberen Höhenklasse 1,7 mal höher eingestuft als dies über alle Höhenlagen der Fall ist. Dagegen gilt für Einheiten innerhalb der unteren Höhenklasse ein um den Faktor 0,4 vermindertes Schälrisiko. Das Chancenverhältnis oder odds ratio (nicht aber das Schälprozent!) erhöht sich also in der einen Klasse um $71 \%$ und verringert sich in der anderen um $56 \%$ (negatives Vorzeichen beim Regressionskoeffizienten). Ist die Variable nicht klassifiziert, bezieht sich der ausgegebene Wert auf jede weitere Einheit, um die der Faktor erhöht wird. Logarithmiert man die einzelnen odds ratios, lassen sich nun wieder die entsprechenden Regressionskoeffizienten der Klassen bilden. Durch das Aufsummieren der absoluten 
Beträge zeigt sich dann - als Gesamtabweichung - der Regressionskoeffizient für den Faktor insgesamt.

\subsubsection{Generalisierte lineare Modelle}

Generalisierte lineare Modelle sind allgemein bekannt. R-Statistics bietet jedoch eine Besonderheit bei der Analyse im GLM. Die abhängige Variable im GLM kann auch bei der logistischen Regression, also wenn die Kategorie „family“ auf „binomial“ gesetzt ist, mit Werten außerhalb der 0-1-Codierung arbeiten: „If a binomial glm model was specified by giving a two-column response, the weights returned by prior.weights are the total numbers of cases (factored by the supplied case weights) and the component $\mathrm{y}$ of the result is the proportion of successes. 47 Von dieser Möglichkeit wird im Rahmen dieser Arbeit Gebrauch gemacht, um die relativen Anteile innerhalb der abhängigen Variablen zu berücksichtigen.

\subsubsection{Verwendete Software}

Für das Datenmanagement, also die Anlage von Datenbanken und die Verschneidung aller eingehender Informationen, wurde Microsoft Access benutzt. Kleinere Berechnungen fanden in Microsoft Excel statt. Zur georeferenzierten Bearbeitung und Darstellung der Daten wurde Arc GIS von ESRI verwendet. Für die statistische Analyse wurde RStatistics benutzt, wobei logistische Regressionen daneben auch in SPSS 16.0.1 gerechnet wurden. Die Diagramme wurden in Origin erstellt. Der Textsatz erfolgte mittels LaTeX.

\footnotetext{
${ }^{7}$ The R Development Core Team (1999)
} 



\section{Charakterisierung der Stichprobenpunkte}

Um zunächst einen Eindruck von den räumlichen Bedingungen der Stichprobenpunkte zu bekommen, werden die Standorte im Folgenden charakterisiert. Entsprechend der Trennung homogen mit Fichten besetzter Stichprobenpunkte von solchen mit heterogener Baumartenausstattung, was für den Eingang in binäre logistische Regressionsmodelle später von Bedeutung ist, werden auch hier für jedes Gebiet jeweils zwei Datensets betrachtet.

\subsection{Lage der Stichprobenpunkte im Gelände}

\subsubsection{Höhenlage}

Zum Zwecke einer ersten Übersicht sind die mittleren Höhen der Inventurpunkte in Abbildung 3.1 dargestellt. In der linken Hälfte der Abbildung wird zwischen homogenen und heterogenen Punkten im Solling unterschieden, in der rechten Hälfte befindet sich die analoge Darstellung für die beiden Datensets des Harzes. Im Vergleich beider Untersuchungsgebiete zeigen sich für den Solling deutlich geringere Werte als für den Harz. In beiden Gebieten liegen die homogenen Stichprobenpunkte im Mittel höher als die heterogenen. Für den homogenen Datenpool des Solling ergibt sich eine mittlere Höhe von 402 m, für den heterogenen eine mittlere Höhe von 384 m. In Tabelle 3.1 sind die entsprechenden Begleitwerte zur Abbildung aufgeführt. Auch im Harz erreicht das homogene, rein mit Fichten besetzte Datenset mit 597 m einen höheren Mittelwert als das heterogen besetzte, dessen Stichprobenpunkte sich im Mittel auf $520 \mathrm{~m}$ Höhe befinden.

\subsubsection{Hangneigung und -richtung des Geländes}

Zunächst ist festzustellen, dass sich die beiden Untersuchungsgebiete in ihren Geländeeigenschaften deutlich unterscheiden. Wie bereits unter 3.1.1 gezeigt wurde, verfügt 
3. Charakterisierung der Stichprobenpunkte

Tabelle 3.1: Mittlere Höhe homogener und heterogener Sets in Solling und Harz; Höhenangaben in Metern über NN

\begin{tabular}{lrrrrrrr}
\hline Datenset & $n$ & $\bar{x}$ & $s$ & $V$ & $V_{r}$ & $x_{\min }$ & $x_{\max }$ \\
\hline Homogene Plots Solling & 894 & 402 & 68 & 0,17 & $0,57 \%$ & 163 & 525 \\
Heterogene Plots Solling & 1.364 & 384 & 72 & 0,19 & $0,51 \%$ & 145 & 524 \\
Homogene Plots Harz & 773 & 597 & 118 & 0,20 & $0,71 \%$ & 283 & 894 \\
Heterogene Plots Harz & 932 & 520 & 112 & 0,22 & $0,71 \%$ & 236 & 864 \\
\hline
\end{tabular}

$n=$ Anzahl, $\bar{x}=$ arithmetisches Mittel der Höhe, $s=$ Standardabweichung, $V=$ Variationskoeffizient, $V_{r}=$ relativer Variationskoeffizient, $x_{\min }=$ Minimalwert, $x_{\max }=$ Maximalwert

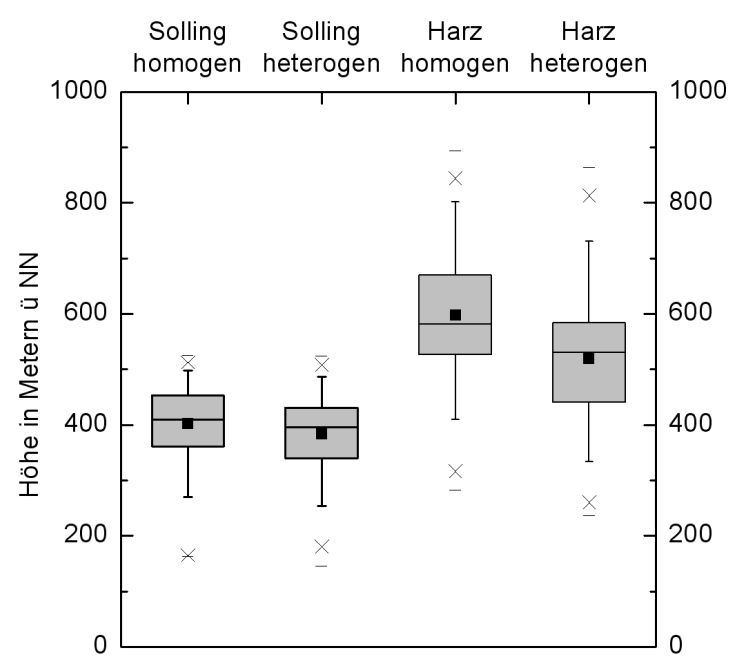

Abbildung 3.1: Mittlere Höhe der Inventurpunkte; dargestellt für homogene und heterogene Datensets in Solling und Harz; Boxpercentile: 25-50-75\%, Whisker: 5-95\%

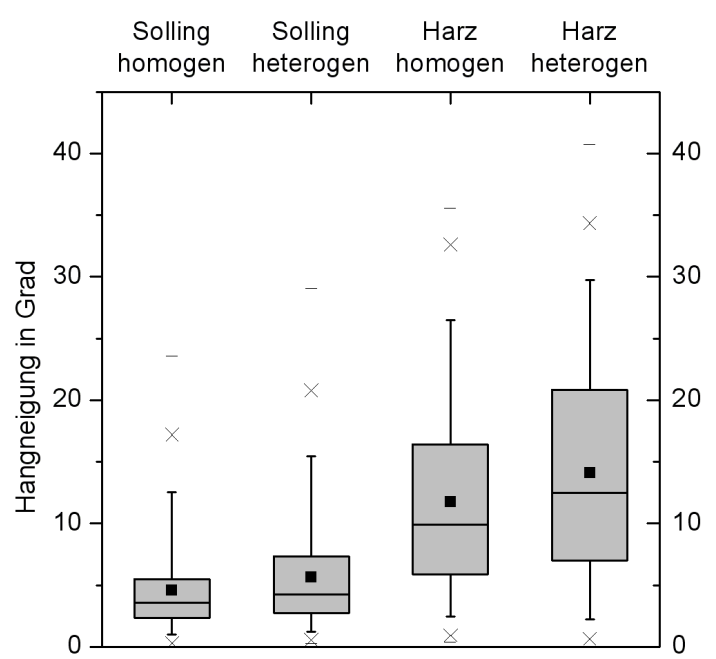

Abbildung 3.2: Mittlere Hangneigung der Inventurpunkte in Grad; dargestellt für homogene und heterogene Datensets in Solling und Harz; Boxpercentile: 25-50-75 \%, Whisker: $5-95 \%$

der Stichprobenumfang des Harzes über Standorte in weitaus höheren Lagen als der des Sollings. Auch hinsichtlich der Reliefenergie wartet der Harz mit höheren Werten auf. So liegt die mittlere Hangneigung der dortigen Stichprobenpunkte bei etwa 13 Grad, während sie im Solling nur rund 5 Grad beträgt. In Tabelle 3.2 ist die mittleren Hangneigung für beide Gebiete aufgeführt, wobei jeweils zwischen homogenen und heterogenen Datensets unterschieden wird. Demnach liegen die Mittelwerte der heterogenen Klassen jeweils etwas über denen der homogenen Klassen des jeweiligen Gebietes. In allen vier Datensets zeigen sich zudem sehr hohe Werte der Variationskoeffizienten. Die Stichprobenpunkte liegen also breit gestreut in unterschiedlichsten 

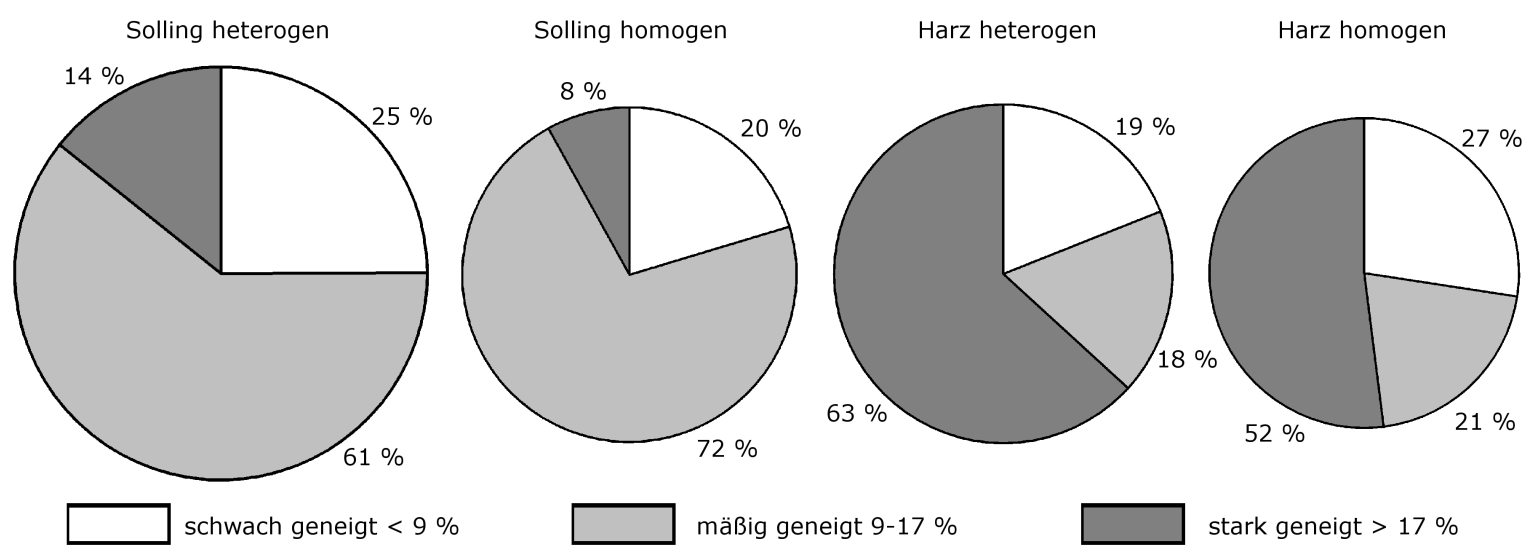

Abbildung 3.3: Anteile der Hangklassen an den Datensets; die Flächengrößen der Kreise entsprechen dem relativen Verhältnis der Stichproben zueinander

Hanglagen. Abbildung 3.2 veranschaulicht die uneinheitlichen Bedingungen zwischen den Gebieten und zeigt die Varianzbreite der einzelnen Datensets. Ebenfalls unterschieden zwischen heterogenen und homogenen Plots beider Gebiete weisen doch alle vier Datengruppen eine Lage des Medians (Querstrich innerhalb der Box) unterhalb des arithmetischen Mittels (Punkt innerhalb der Box) auf. Einzelne Ausreißer nach oben, also hin zu deutlich höheren Hangneigungswerten, sorgen für diese Verschiebung. Der Großteil der Stichprobenpunkte liegt demnach unterhalb des angegebenen Mittelwertes.

Die Einteilung der Hangneigung erfolgt zunächst in Anlehnung an den Arbeitskreis Forstliche Standortskartierung. Zur Klassifizierung der Hangneigung wird dort zwischen „flach, mäßig und stark steigenden Hängen“ unterschieden. Flächen mit weniger als 9 \% Steigung gelten hierbei als höchstens „,schwach geneigt“, Flächen mit mehr als 17 \% Steigung als mindestens „stark geneigt“ (AKS 1980 zitiert in ScHULz 2003). In

Tabelle 3.2: Mittlere Hangneigung homogener und heterogener Sets in Solling und Harz; Angabe der Neigungswerte in Grad

\begin{tabular}{lrrrrrrr}
\hline Datenset & $n$ & $\bar{x}$ & $s$ & $V$ & $V_{r}$ & $x_{\min }$ & $x_{\max }$ \\
\hline Homogene Plots Solling & 894 & 4,6 & 3,6 & 0,78 & $2,62 \%$ & 0 & 23,6 \\
Heterogene Plots Solling & 1.364 & 5,7 & 4,5 & 0,79 & $2,14 \%$ & 0,3 & 29,1 \\
Homogene Plots Harz & 773 & 11,7 & 7,6 & 0,65 & $2,34 \%$ & 0,4 & 35,6 \\
Heterogene Plots Harz & 932 & 14,1 & 8,7 & 0,62 & $2,02 \%$ & 0 & 40,7 \\
\hline
\end{tabular}

$n=$ Anzahl, $\bar{x}=$ arithmetisches Mittel, $s=$ Standardabweichung, $V=$ Variationskoeffizient, $V_{r}=$ relativer Variationskoeffizient, $x_{\min }=$ Minimalwert, $x_{\max }=$ Maximalwert 
die Modelle zur binären logistischen Regressionsrechnung geht der Parameter in dieser Einteilung ein, sodass mit Abbildung 3.3 zunächst die relativen Anteile dieser drei Klassen verdeutlicht werden sollen. Unter Berücksichtigung der Trennung heterogener von homogenen Stichproben, ergeben sich wiederum vier Datensets. Entsprechend der bereits in Abbildung 3.2 zu erkennenden Mittelwertunterschiede beider Untersuchungsgebiete heben sich auch hier die Werte des Harzes mit größeren Anteilen der steileren Klassen deutlich von denen des Sollings ab. Die Klasse der schwachen Steigungen nimmt hingegen in allen Datensets annähernd den gleichen Anteil ein, sodass die steile Hangklasse im Harz ihre höheren Anteile aus der mittleren bezieht. Das Schaubild vermittelt darüber hinaus einen Größenvergleich der vier Stichproben zueinander, da die jeweilige Fläche der Kreisdiagramme in Relation zu den übrigen gesetzt wurde.

Die Abbildungen 3.4 und 3.5 veranschaulichen die Klassenanteile für homogene Fichtenplots in Harz und Solling. Im Harz sind fünf der acht Klassen recht gleichmäßig mit Stichprobenpunkten besetzt. Standorte mit Nord-, Nordost- oder Ostexposition sind im Datenset jedoch schwächer vertreten. Im Solling hingegen dominieren drei Expositionsklassen gegenüber den restlichen fünf Himmelsrichtungen. West-, Nordwest- und Nordexpositionen nehmen zusammen über $50 \%$ der Stichprobe ein. Die Datensets der heterogenen Stichprobenpunkte zeigen für den Harz (Abb. 3.6) eine fast ausgeglichene Verteilung auf die Expositionen. Lediglich die Klasse der Ostausrichtung bleibt etwas hinter den anderen zurück. Im Solling (Abb. 3.7) dominieren wiederum westliche und nordwestliche Expositionen.

Differenziert man die Hangrichtungsanteile nach Klassen unterschiedlicher Hangneigung, ergeben sich detaillierte Betrachtungen. In Abbildung 3.8 sind zunächst die Klassenanteile für heterogene Plots dargestellt. Die Hangneigungsklassen nehmen von links (schwach) nach rechts (stark) an Steigung zu. Zu beachten gilt, dass die Skalierung der Diagramme, je nach Maximalwert des Datensets, bei $20 \%$ - oder bei $30 \%$-Anteil endet. Ungeachtet einer möglichen späteren Fokussierung auf die Klassen stärkerer Steigung sollen hier zunächst alle Klassen nebeneinander betrachtet werden. In der oberen Reihe sind die Werte des Sollings dargestellt. Die Verteilung der Stichprobenpunkte auf die acht Hangrichtungsklassen zeigt bei gesonderter Berücksichtigung der Neigung, dass Stichprobenpunkte schwach geneigter Standorte einen Überhang an westlich bis nördlicher Ausrichtung haben. Westliche Hangrichtungen überwiegen auch leicht bei mittlerer Neigung (Abbildung in der Mitte), wobei die Verteilungs ich insgesamt recht ausgeglichen darstellt. Von den Stichprobenpunkten an stark geneigten Hängen befin- 
den sich die meisten an südlich bis nordwestlich ausgerichteten Standorten (Abbildung rechts).

Im Harz (untere Reihe Abb. 3.8) lassen sich kaum allgemeine Aussagen treffen. Stichprobenpunkte an schwach und mäßig geneigten Hängen sind zwar nicht auf alle Richtungen gleichmäßig verteilt, zeigen aber keinen Trend bei den einzelnen Ausreißern.

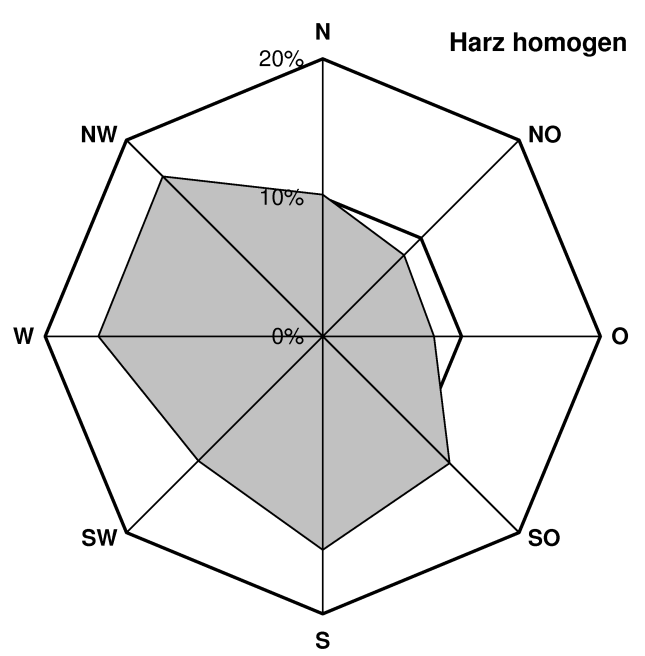

Abbildung 3.4: Verteilung der Inventurpunkte in Klassen unterschiedlicher Exposition; dargestellt als Relativwert für homogene Plots im Harz; $n=773$

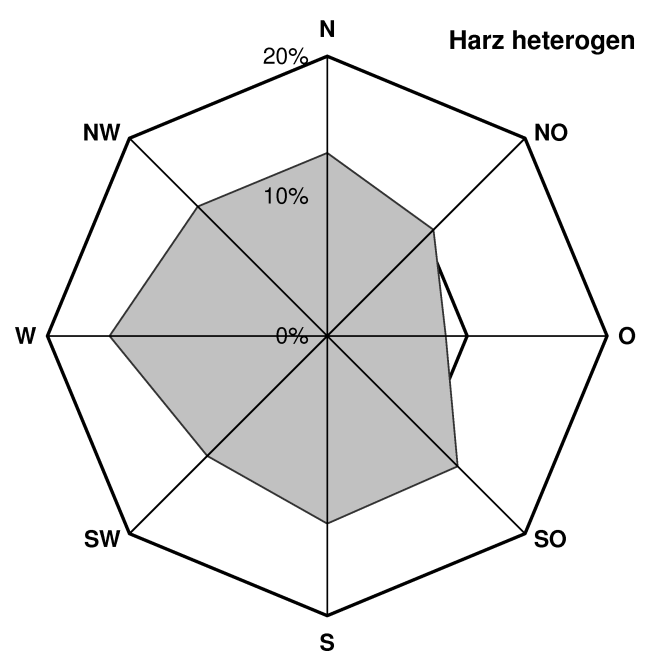

Abbildung 3.6: Verteilung der Inventurpunkte in Klassen unterschiedlicher Exposition; dargestellt als Relativwert für heterogene Plots im Harz; $n=932$

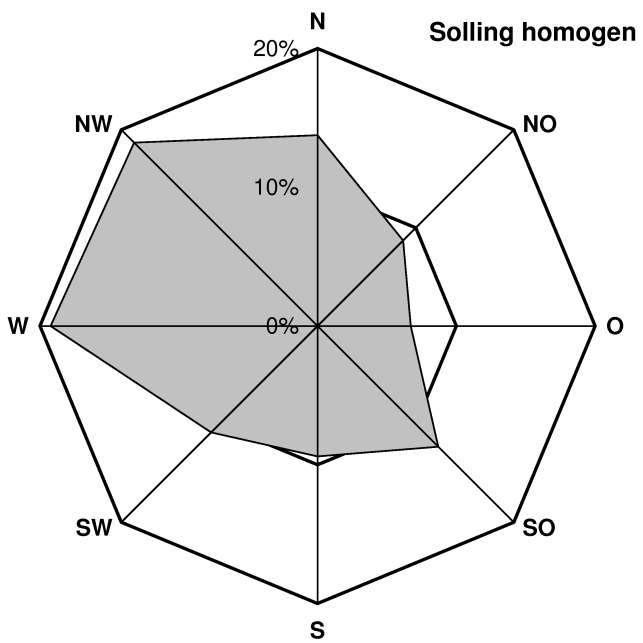

Abbildung 3.5: Verteilung der Inventurpunkte in Klassen unterschiedlicher Exposition; dargestellt als Relativwert für homogene Plots im Solling; $n=891$ ( $+3 x$ Plateau $)$

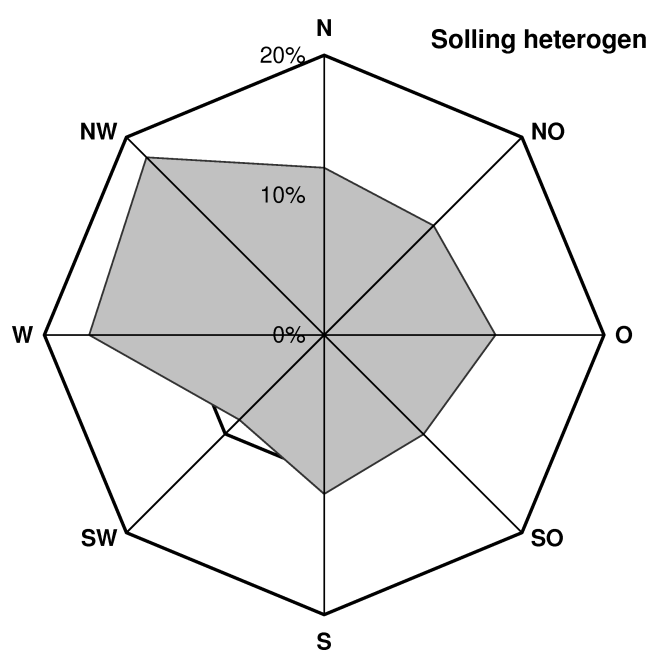

Abbildung 3.7: Verteilung der Inventurpunkte in Klassen unterschiedlicher Exposition; dargestellt als Relativwert für heterogene Plots im Solling; $n=1.364$ 

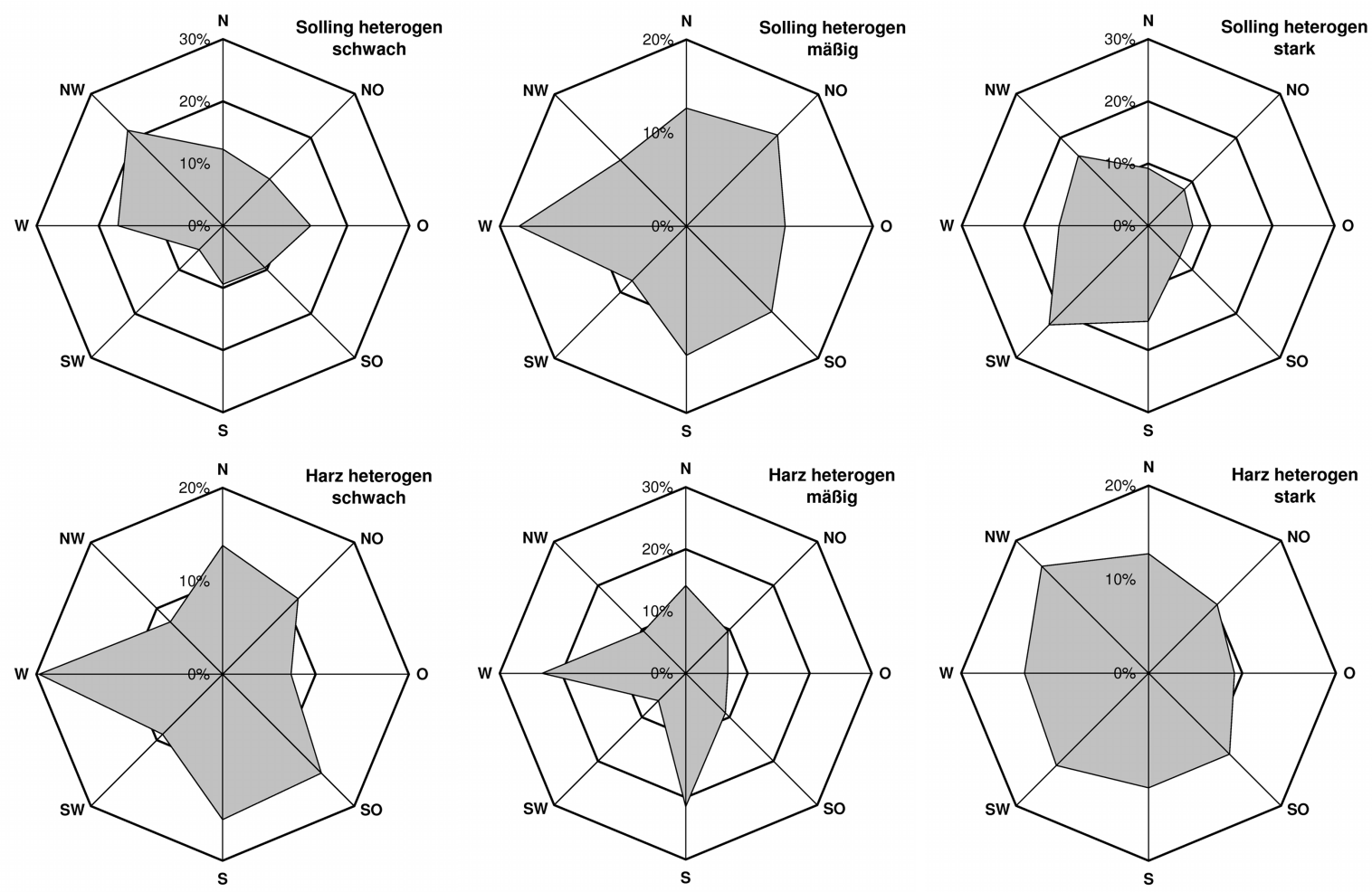

Abbildung 3.8: Verteilung der Inventurpunkte in Klassen unterschiedlicher Exposition in Abhängigkeit von Hangneigungsklassen (von links: schwach, mäßig, stark steigendes Gelände); dargestellt als Relativwert für heterogene Plots im Solling (obere Reihe) und im Harz (untere Reihe)

Südliche und westliche Hangrichtungen nehmen allerdings größere Anteile des Stichprobenumfangs ein. Bei den stark geneigten Hängen kommen alle Richtungen etwa gleich häufig im Datenpool vor.

In Abbildung 3.9 sind nun die Klassenanteile für homogene Plots dargestellt. Auch hier nehmen die Hangneigungsklassen von links (schwach) nach rechts (stark) an Steigung zu. Die Skalierung der Diagramme variiert wiederum mit Endwerten von 20 \%oder 30 \% . Die Werte für den Solling, in der oberen Reihe dargestellt, zeigen ein sehr uneinheitliches Bild. Während bei der Klasse schwacher Steigung nordwestliche Expositionen allein vorherrschen, kommen beim Datenset der mäßigen Steigungsklasse auch solche mit südöstlicher Orientierung hinzu. Die Stichprobenpunkte auf stark ansteigendem Gelände, dargestellt in der rechten Grafik, befinden sich hingegen fast ausschließlich an Orten mit südöstlicher bis westlicher Exposition ${ }^{1}$ und zeigen die stärkste Klumpung aller Datensets.

\footnotetext{
${ }^{1}$ Aussagen über Bereiche mit „von ... bis ... “ orientieren sich am Uhrzeigersinn.
} 

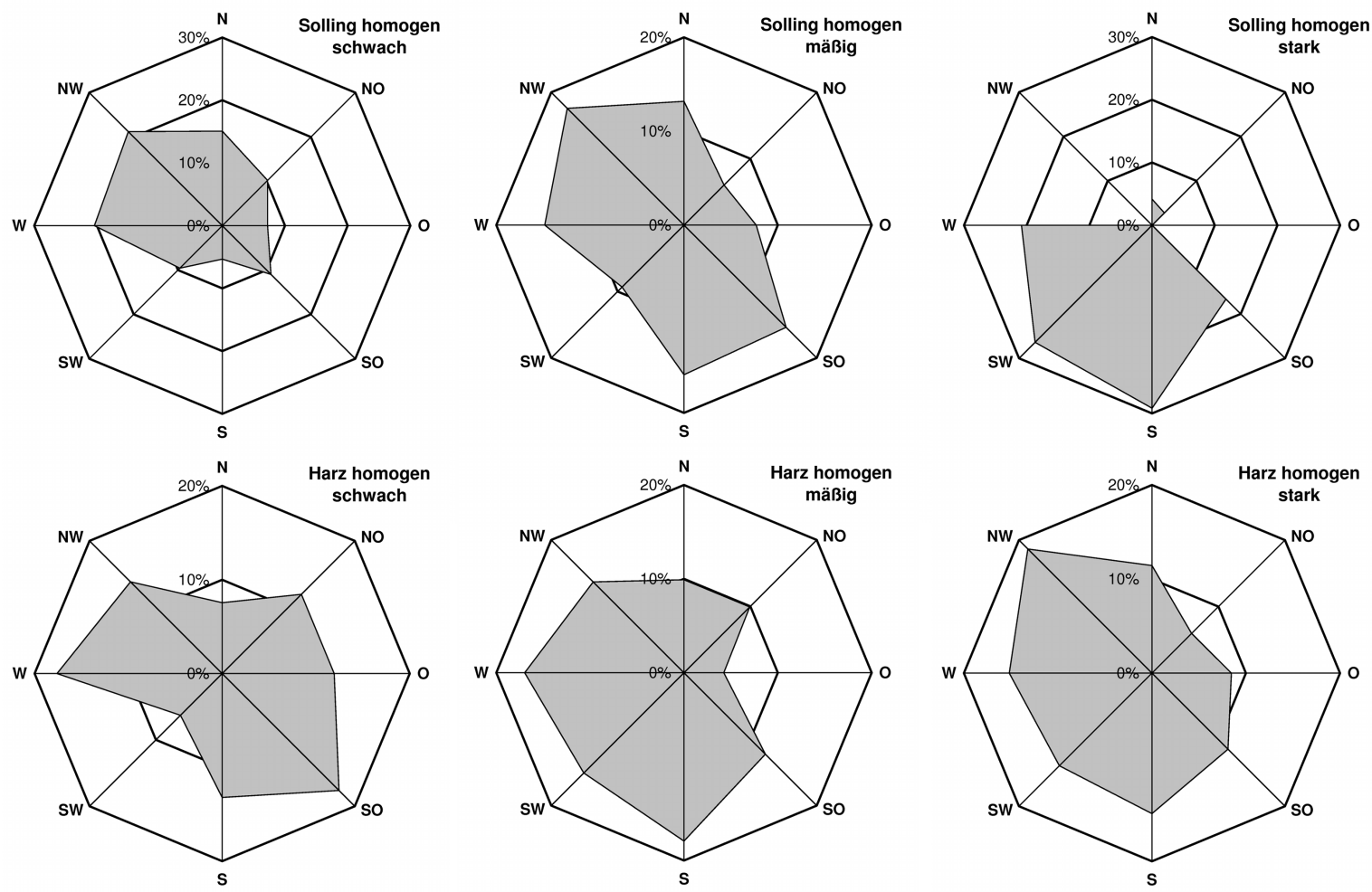

Abbildung 3.9: Verteilung der Inventurpunkte in Klassen unterschiedlicher Exposition in Abhängigkeit von Hangneigungsklassen (von links: schwach, mäßig, stark steigendes Gelände); dargestellt als Relativwert für homogene Plots im Solling (obere Reihe) und im Harz (untere Reihe)

Etwas gleichmäßiger verteilt stellen sich die Anteile an den Expositionsklassen im Harz dar (untere Reihe). Beim Datenset mit schwacher Steigung am Standort entfallen lediglich auf die Klassen nördlicher und südwestlicher Richtung weniger Punkte als auf die übrigen Klassen. Bei den Stichprobenpunkten der mäßig steilen Hänge überwiegen jedoch deutlich die südlichen bis westlich Richtungen, mit einem Tiefstwert für nach Osten gerichteter Standorte. Diese Tendenz bleibt auch bei der dritten Hangneigungsklasse erhalten, doch nimmt dort zusätzlich die nordwestliche Richtung einen erheblichen Anteil des Probenumfangs ein.

\subsubsection{Exponiertheit im Gelände}

Wie unter 2.3.2 auf Seite 23 erläutert, steht für das Maß der Exponiertheit im Gelände ein digitales Rechenmodell zur Verfügung, welches mittels fiktivem Lichtstrahl den Freistand eines Punktes im Gelände bewertet. Hierdurch ergeben sich 37 Expositionsklassen, deren relativen Anteile in den Abbildungen 3.10 und 3.11 grafisch dargestellt sind. 
Die Werte der homogenen und heterogenen Stichprobenpunkte eines Untersuchungsgebietes sind jeweils gemeinsam in einem Diagramm abgebildet. Zur besseren optischen Differenzierung der Linien ist für die homogenen Datensets jeweils die Fläche unter der Kurve dargestellt.

Für den Solling (Abb. 3.10 ergeben sich keine nennenswerten Unterschiede bei der Betrachtung der Klassenanteile homogener und heterogener Plots. Der Verlauf beider Linien zeigt einen Höhepunkt bei den Klassen 20 bis 24 und einen überproportional großen Anteil der Klasse mit dem Wert 36. Auch im Harz (Abb. 3.11) ist der Linienverlauf der homogenen Stichprobenpunkte weitestgehend vergleichbar mit dem der heterogenen. Lediglich in den Klassen 0 bis 6 unterscheiden sich beide Datensets deutlich. Während die Klassenwerte homogener Plots in diesem Bereich, ausgehend von 0, von sehr niedrigem Niveau aus leicht ansteigen, zeigt sich beim Set der heterogenen Stichprobe hier ein sehr hoher Wert mit anschließendem Abfall der relativen Anteile. Immerhin $10 \%$ der heterogenen Punkte werden demnach der Klasse 0 zugewiesen, in welche beim Datenset der homogenen Punkte nur $3 \%$ fallen. Insgesamt unterscheiden sich die beiden Untersuchungsgebiete sehr viel deutlicher voneinander, als die jeweiligen Datensets der Gebiete untereinander. So fehlen im Harz stark exponierte Standorte mit dem Wert 36 völlig, während sie im Solling die größte Klasse stellen. In nennenswertem Umfang kommen im Harz ohnehin nur Werte bis 23 vor. Entsprechend größer fällt hier der Anteil schwach bis gar nicht exponierter Standorte aus. Die Anteile der Exponiertheitswerte spiegeln also insgesamt die stärkere Zerklüftung des Harzes gegenüber dem Solling mit seinen höheren Anteilen von Plateaulagen wider. Zudem befinden sich

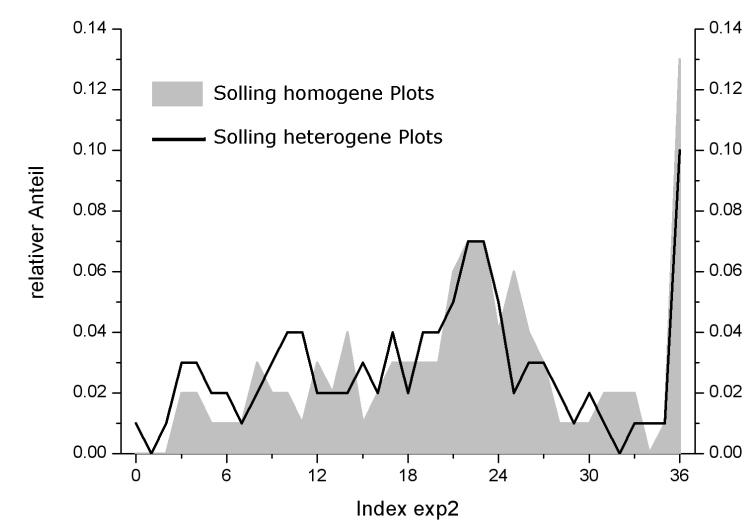

Abbildung 3.10: Exponiertheit homogener und heterogener Plots im Solling; Vergleich der relativen Anteile

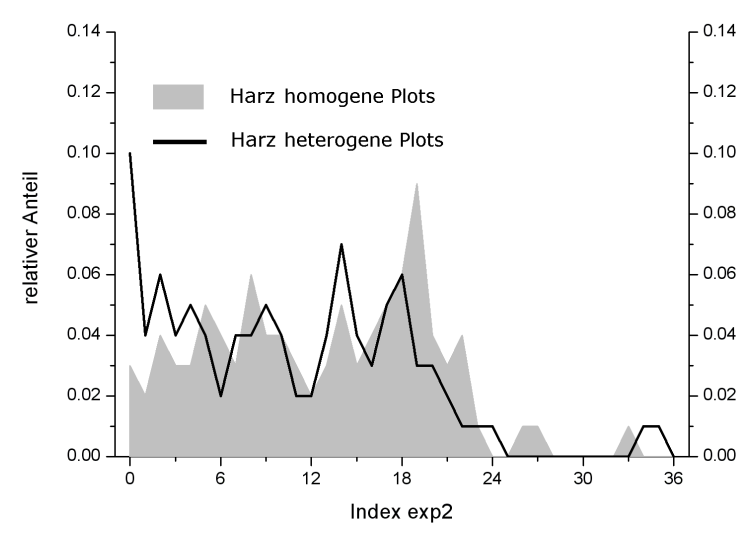

Abbildung 3.11: Exponiertheit homogener und heterogener Plots im Harz; Vergleich der relativen Anteile 


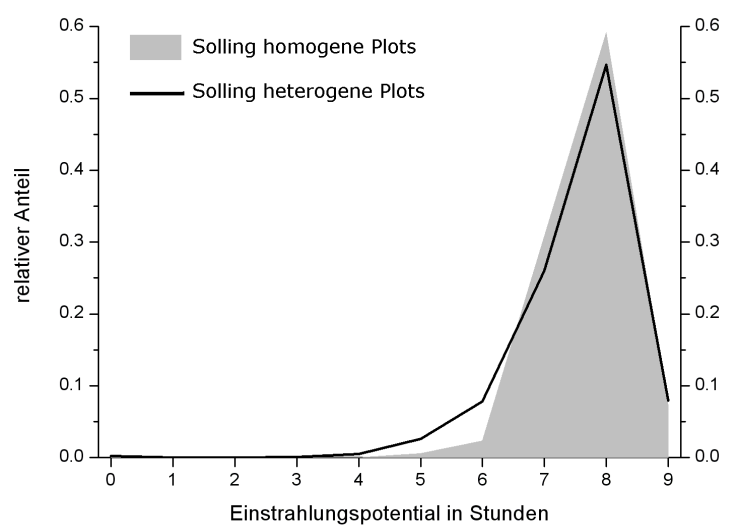

Abbildung 3.12: Potentieller Strahlungsinput homogener und heterogener Plots im Solling; Vergleich der relativen Anteile

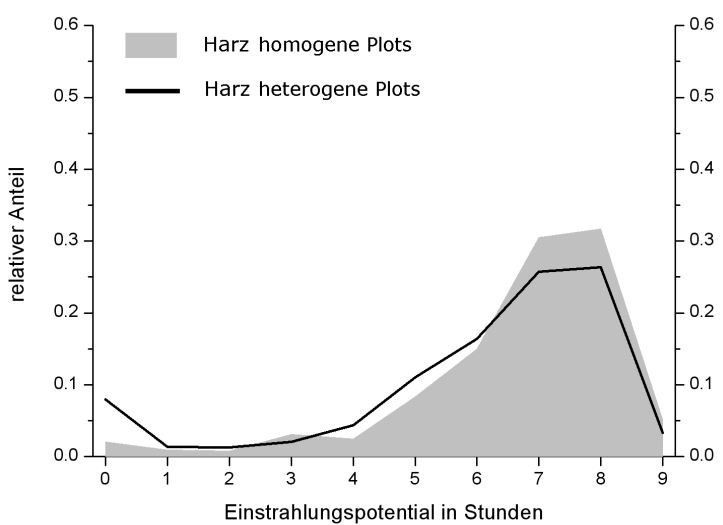

Abbildung 3.13: Potentieller Strahlungsinput homogener und heterogener Plots im Harz; Vergleich der relativen Anteile

in den Kerbtälern des Harzes offenbar mehr heterogenbesetzte Stichprobenpunkte mit Mischbaumarten, während reine Fichtenstichproben dort seltener sind.

\subsubsection{Einstrahlungspotential am Stichprobenpunkt}

Im Sinne einer einheitlichen Darstellungsweise sind die Anteile der Einstrahlungsklassen in den Abbildung 3.12 und 3.13 trotz geringerer Klassenanzahl ebenfalls als Liniendiagramme ausgeführt und die Werte der homogenen und heterogenen Datensets wiederum zusammengelegt. Wie in den Abbildungen 3.11 und 3.10 ist auch hier für die homogenen Plots die Fläche unter der Kurve ausgewiesen. Die Werte geben die relativen Anteile der Klassen 0 bis 9 am jeweiligen Datenset wider. In beiden Gebieten verlaufen die Kurven der homogenen und heterogenen Datensets annähernd parallel. Bis auf kleinste Abweichungen im Kurvenverlauf ist lediglich im Harz ein eindeutiger Unterschied in den Anteilen der Klasse mit dem Wert 0 zu verzeichnen. Hier erreicht der heterogene Datenpool Werte um $8 \%$, während der homogene auf etwa $2 \%$ kommt. Im Vergleich der Gebiete miteinander wird ein flacherer Anstieg der Strahlungswerte im Harz gegenüber dem Solling deutlich. In beiden Gebieten stellen die Klassen 7 und 8 jedoch jene mit den größten Anteilen an den Stichproben dar. 


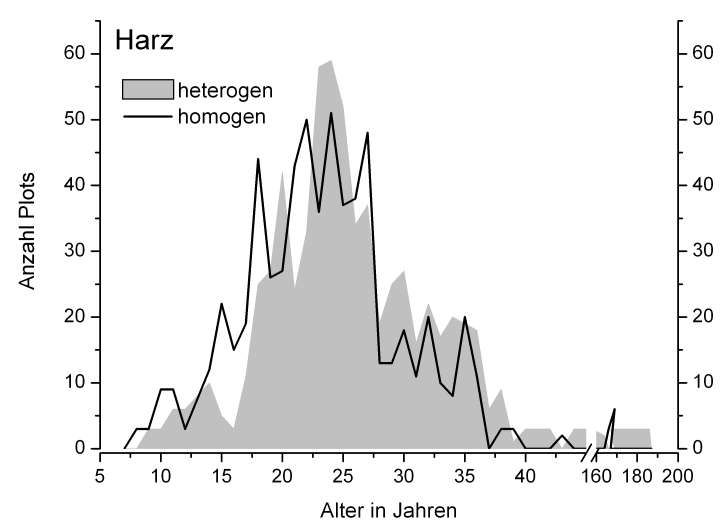

Abbildung 3.14: Verteilung des Bestandesalters der Probeflächen im Harz; Vergleich der relativen Anteile

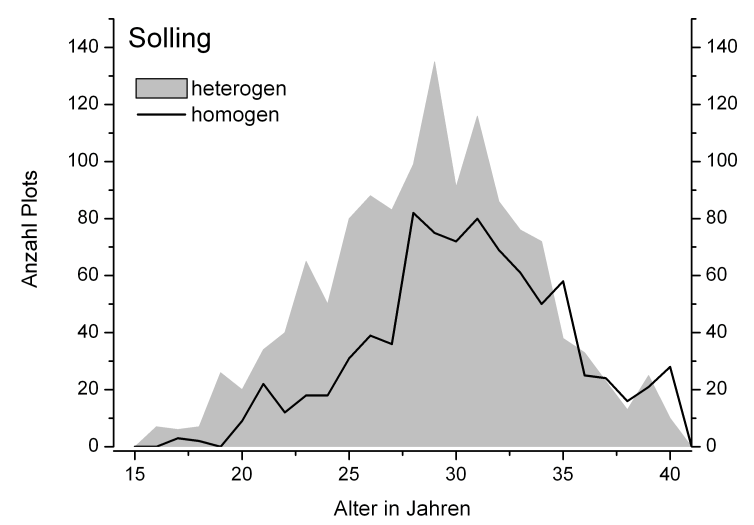

Abbildung 3.15: Verteilung des Bestandesalters der Probeflächen im Solling; Vergleich der relativen Anteile

\subsection{Bestandesparameter am Stichprobenpunkt}

\subsubsection{Bestandesalter}

Aufgrund der Zweckgebundenheit der Inventur zur Schälschadenskartierung fand bereits durch die Aufnahme der Stichprobenpunkte eine Auswahl hinsichtlich des Alters der Bestände statt (vgl. 2.2.1, S. 13). Die durch die Inventur erfassten Altersklassen befinden sich entsprechend der Zielvorgabe in einem Bereich hohen Schälrisikos. Als arithmetisches Mittel wird für heterogene Stichprobenpunkte ein Alter von 42 Jahren ausgewiesen, während es im homogenen Datenpool 27 Jahre beträgt. Die Standardabweichung $s$ ist mit 22 (homogen) und 40 (heterogen) in beiden Fällen sehr hoch. Der gegen Extremwerte deutlich robustere Median liegt dagegen mit 24 und 26 in beiden Stichproben auf vergleichbarem Niveau. Als Modalwert wird in beiden Varianten das Alter 24 ermittelt.

Die in den Stichproben des Harzes vorkommenden hohen Alterswerte fehlen im Datenumfang des Solling völlig. Dort liegt der Maximalwert in beiden Stichproben bei einem Bestandesalter von 40 Jahren. Alle Werte weisen auf eine enge Klumpung um die Mittelwerte hin. Diese liegen bei $31(s=4,7)$ für das homogene und $29(s=4,8)$ für das heterogene Set und unterscheiden sich damit nur geringfügig von Median und Modus.

Um die Verteilung der Altersdiversität zu veranschaulichen, ist diese in den Abbildungen 3.14 und 3.15 für jeweils beide Datensets gesondert abgetragen. Bedingt durch die Extremwerte im Harz wurde die Abzisse in Abbildung 3.14 zwischen den Werten 
50 und 160 ausgesetzt. Für jedes Untersuchungsgebiet ist der Verlauf der homogenen Stichprobenpunkte als Fläche unter der Kurve dargestellt, während jener der heterogenen als Linie darübergelegt ist. In ihrem Verlauf ähneln sich die Verteilungskurven sowohl innerhalb der Gebiete als auch zwischen einander und folgen einer Normalverteilung.

\subsubsection{Bestandestypen und Baumartenzahl}

Abbildung 3.16 auf Seite 46 zeigt den Anteil der verschiedenen Bestandestypen an der Verteilung der gesamten Fichten-Stichprobenpunkte in den beiden Untersuchungsgebieten. Hierin sind also alle Plots enthalten, unter deren aufgenommenen Bäumen sich mindestens eine Fichte befindet und die zu einer Analyse mit metrischem Skalenniveau der abhängigen Variablen herangezogen werden können. Auch hier bleibt der Anteil der Fichtenreinbestände am größten, doch wird er in deutlichem Maße auch von anderen Typen ergänzt. Vor allem in Mischbeständen mit der Fichte oder der Buche als Leitart liegt ein weiterer Teil der Stichprobenpunkte. In der Abbildung sind diese zusammengefasst als „Fichte“ und „Buche“ gekennzeichnet, alle weiteren BT-Klassen wurden kumuliert in „Rest“ dargestellt. Mit insgesamt rund $31 \%$ fällt demnach der Anteil der Fichtenmischbestände am Datenpool im Solling deutlich umfangreicher aus, als mit etwa $22 \%$ im Harz. Wie sich die Häufigkeitsverteilung der Fichte innerhalb der Stichprobenpunkte darstellt, wurde in Abbildung 2.4 auf Seite 16 gezeigt. Die weitere Betrachtung der Baumartenzahl soll jedoch nicht an der Differenzierung der Stichprobenpunkte nach Bestandestypen festhalten, sondern stattdessen zwischen reinen Fichtenplots (homogen) und Mischplots (heterogen) unterscheiden.

Um eine Übersicht über die Baumartenanzahl der weiteren Umgebung zu bekommen, wurden die Abteilungen hinsichtlich ihrer Baumartenausstattung in einfachen Schritten klassifiziert. Gesondert dargestellt für Solling und Harz zeigen die Abbildungen 3.17 und 3.18 die Verteilung der Stichprobenpunkte auf diese Klassen. Auffällig ist zunächst, dass im Solling kaum Stichprobenpunkte in Abteilungen mit nur einer Baumart lokalisiert sind (1,3\%). Auch Abteilungen mit zwei Baumarten kommen mit einem Anteil von 4,0 \% nur selten vor. Im Harz befinden sich hingegen 7,5\% aller Punkte in Abteilungen mit nur einer Baumart und weitere 10,1 \% in solchen mit zwei Baumarten. Der bei der Auswahl der Plots in Abbildung 3.16 gezeigte Schwerpunkt auf den Bestandestyp 50 spiegelt sich in dieser Auswertung erneut wider. Allerdings nehmen die Klassen mit 4 und 5 Baumarten im Harz den größten Anteil der Stichprobenpunkte ein und stehen 


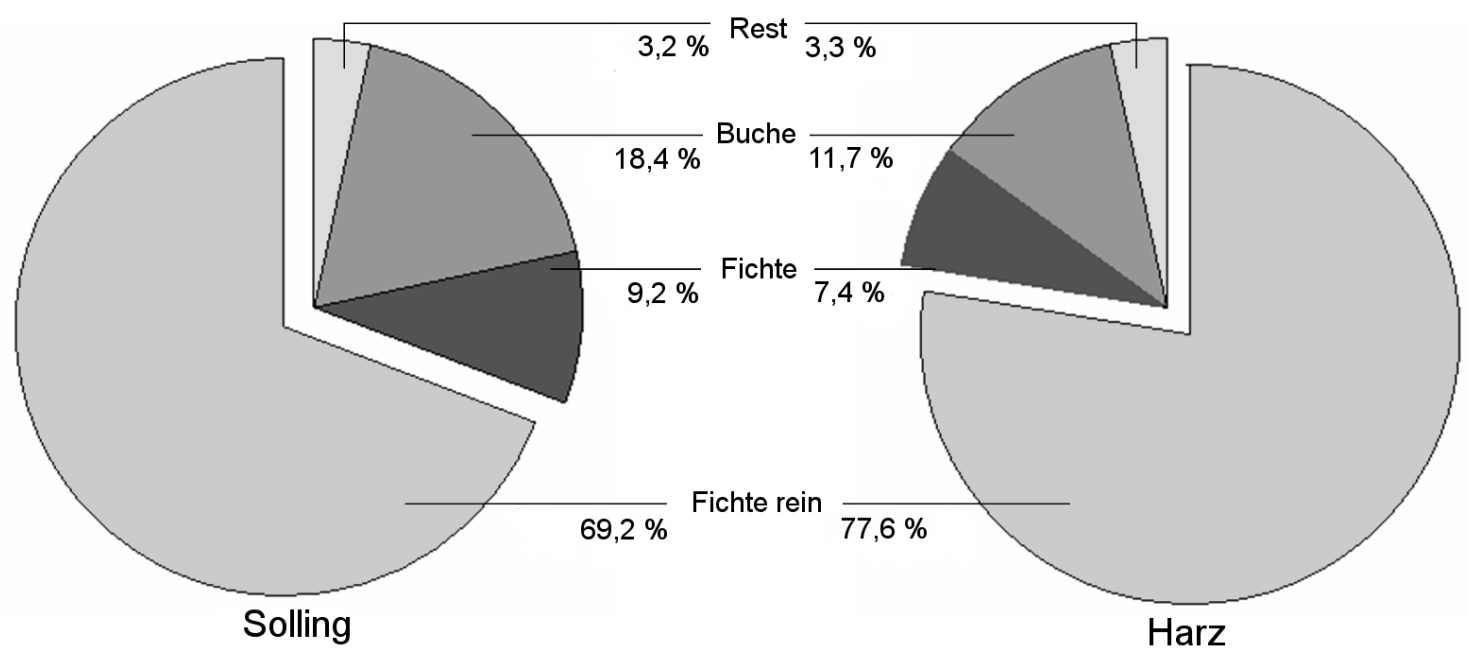

Abbildung 3.16: Relative Anteile der Bestandestypengruppen am Stichprobenumfang von Plots mit mindestens einer Fichte. Der BT 50 wird unter "Fichte rein" als einziger Typ gesondert ausgewiesen. "Fichte" fasst die Typen 52, 53, 54, 56 und 57 zusammen; "Buche" die Typen 21, 23, 24, 25, 26 und 28. Alle anderen Bestandestypen, sowie Stichprobenpunkte ohne Angabe des BT, werden durch „Rest" dargestellt.

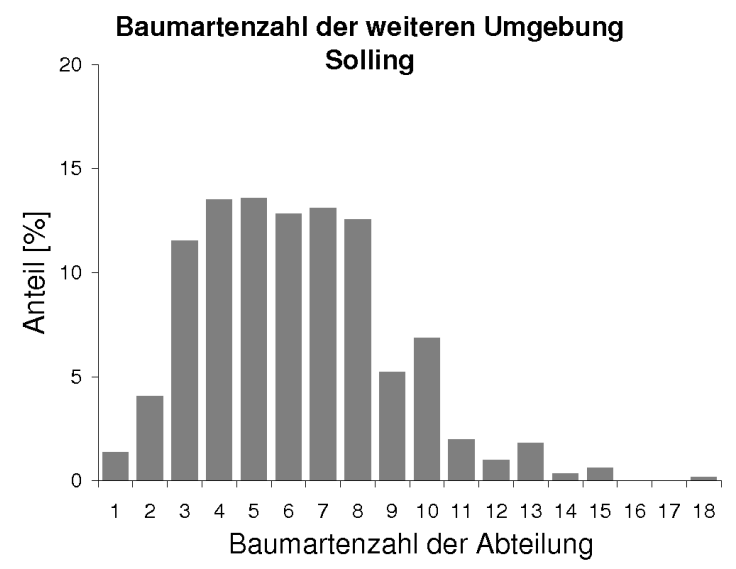

Abbildung 3.17: Verteilung der Inventurpunkte in Klassen unterschiedlicher Baumartenzahl je Abteilung; dargestellt als Relativwert für den Solling; $n=2258$

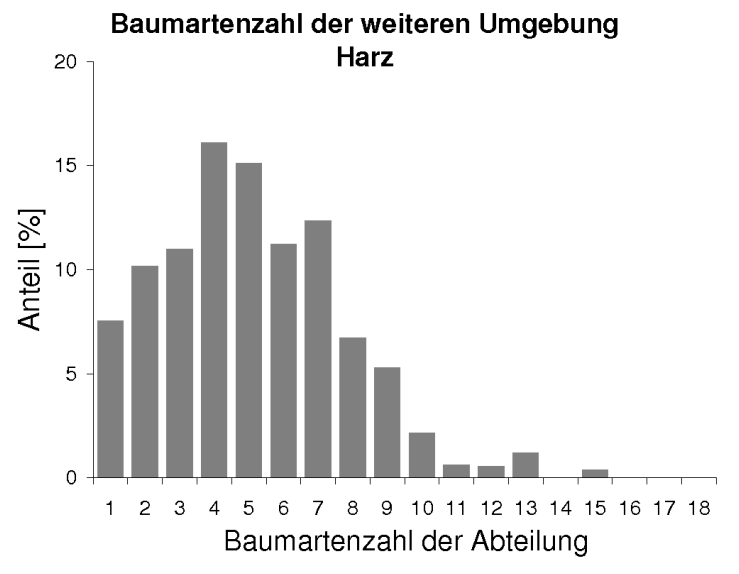

Abbildung 3.18: Verteilung der Inventurpunkte in Klassen unterschiedlicher Baumartenzahl je Abteilung; dargestellt als Relativwert für den Harz; $n=1705$

aus der annähernd gleich verteilten Klassenbreite von 2 bis 7 Baumarten leicht hervor. Im Mittel (Median) finden sich im Harz 5 Baumarten innerhalb der weiteren Umgebung eines Stichprobenpunktes, während es im Solling 6 Baumarten sind.

Analog zu den in den Abbildungen 3.17 und 3.18 dargestellten Anteilen baumartenreicher Abteilungen an der Verteilung der Plots veranschaulichen die Abbildungen 3.19 und 3.20 dies auf kleinerer Ebene. Dargestellt ist auch hier, mit welcher Häufigkeit 


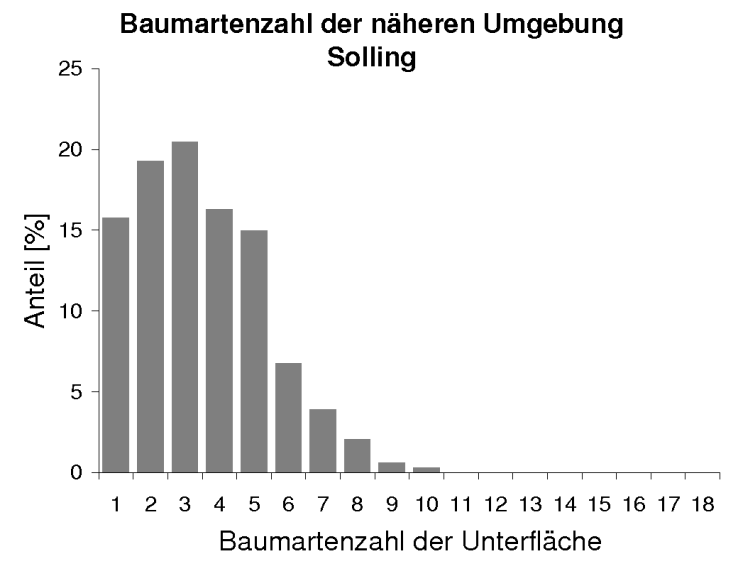

Abbildung 3.19: Verteilung der Inventurpunkte in Klassen unterschiedlicher Baumartenzahl je Unterfläche; Relativwert für den Solling

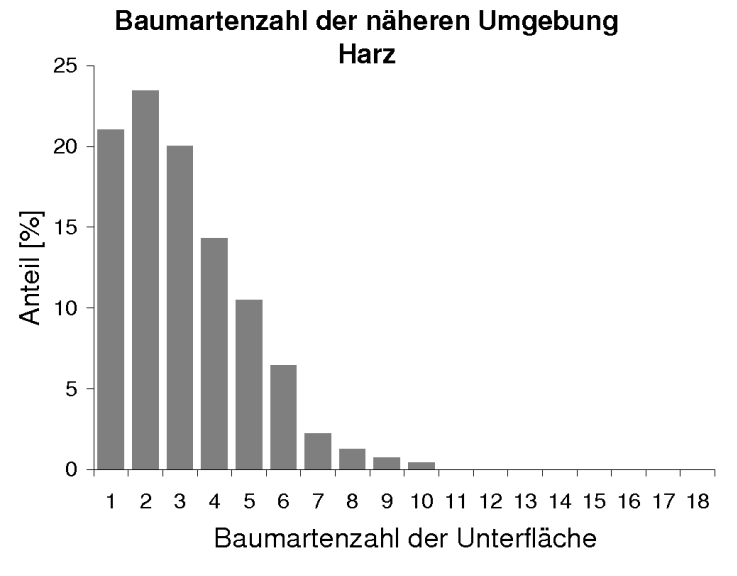

Abbildung 3.20: Verteilung der Inventurpunkte in Klassen unterschiedlicher Baumartenzahl je Unterfläche; Relativwert für den Harz

Stichprobenpunkte der Inventur in Umgebungen mit unterschiedlich vielen Baumarten platziert wurden. Für beide Untersuchungsgebiete zeigt sich bei einer kleineren Referenzfläche auch eine deutliche Abnahme der Baumartenzahl auf dieser betrachteten Fläche. Der hohe Anteil der Fichtenreinbestände innerhalb der Harz-Inventur wirkt sich auch hier auf das vergleichende Ergebnis beider Gebiete aus. Die Klasse der Reinbestände liegt im Harz mit einem Anteil von 21,0 \% erneut über der im Solling mit 15,7 \%. Die höchste Repräsentanz haben im Harz jedoch Flächeneinheiten mit zwei Baumarten und auch in die Klassen mit drei Baumarten fallen viele Aufnahmepunkte.

\subsection{Jagdstreckenaufkommen im Referenzgebiet}

\subsubsection{Zeitliche Verteilung der Rotwilderlegungen}

Zu Beginn der Verwendung von Streckendaten zur Analyse von Schälschadensereignissen sollen die zeitlichen und räumlichen Eigenarten im Aufkommen der Jagdstrecke beider Gebiete betrachtet werden. Der Bejagungszeitraum des Rotwildes ist zumächst durch die Bundes- und Landesjagdzeiten geregelt. Die zeitliche Verteilung der Erlegungen innerhalb der gesetzlichen Jagdzeiten variieren jedoch. Sie sind zusammen mit der Erfassung von Fallwild für beide Untersuchungsgebiete in Abbildung 3.25 dargestellt. Gezeigt wird das Aufkommen der Streckenmeldungen beim Rotwild vom 01.10.2001 bis zum 30.09.2006. Die zugrunde liegenden Tageswerte sind monatsweise zusammenge- 


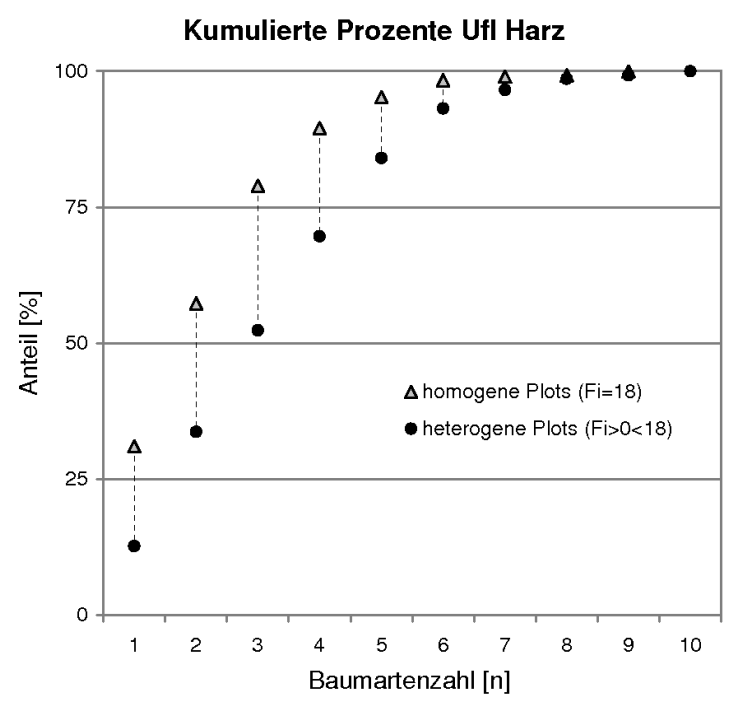

Abbildung 3.21: Kumulierte Prozente der Baumartenzahl je Unterfläche am Anteil der Inventurpunkte; getrennt dargestellt für heterogene (mind. eine, max. 17 Fichten) und homogene Plots (18 Fichten) im Harz

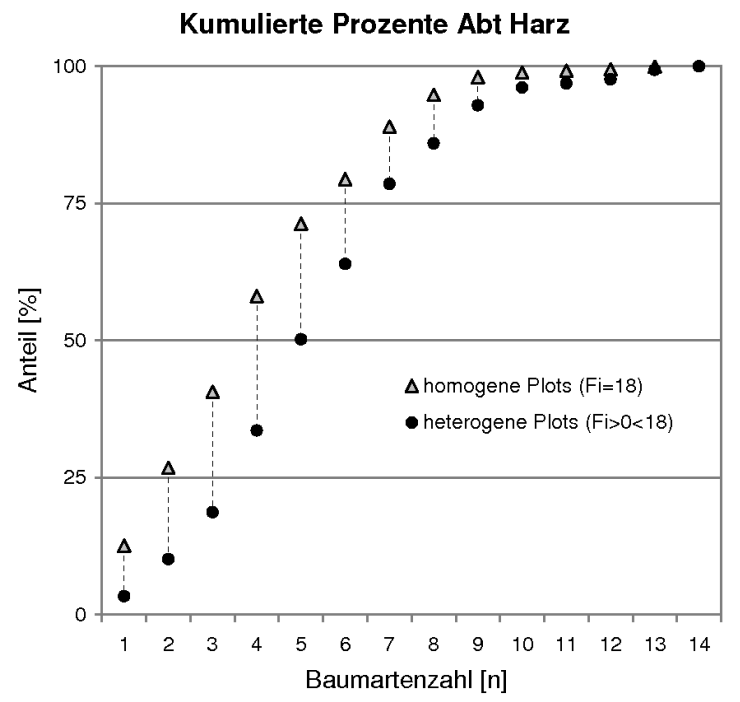

Abbildung 3.23: Kumulierte Prozente der Baumartenzahl je Abteilung am Anteil der Inventurpunkte; getrennt dargestellt für heterogene (mind. eine, max. 17 Fichten) und homogene Plots (18 Fichten) im Harz

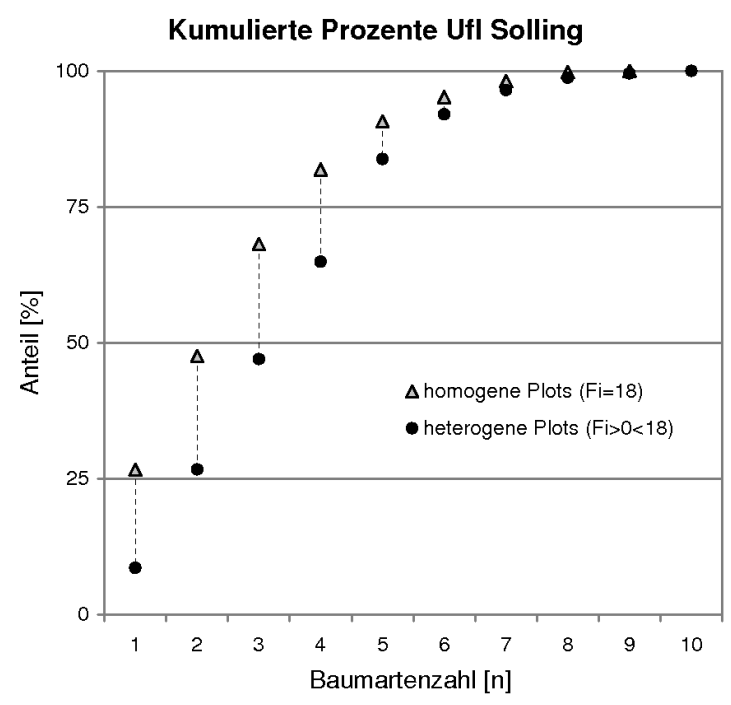

Abbildung 3.22: Kumulierte Prozente der Baumartenzahl je Unterfläche am Anteil der Inventurpunkte; getrennt dargestellt für heterogene (mind. eine, max. 17 Fichten) und homogene Plots (18 Fichten) im Solling

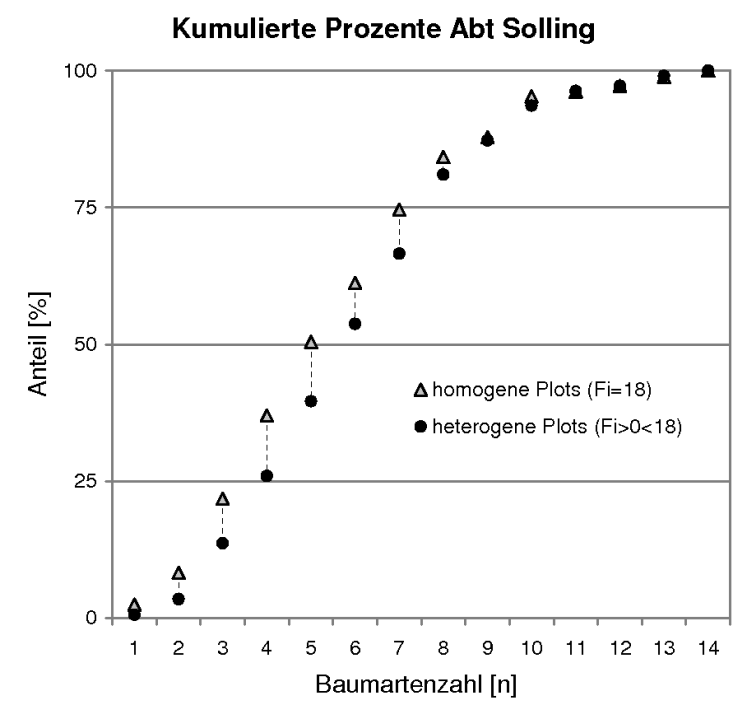

Abbildung 3.24: Kumulierte Prozente der Baumartenzahl je Abteilung am Anteil der Inventurpunkte; getrennt dargestellt für heterogene (mind. eine, max. 17 Fichten) und homogene Plots (18 Fichten) im Solling

fasst und jeweils über das gesamte Gebiet summiert dargestellt. Durch die Ausweisung der absoluten Werte wird das erheblich höhere Streckenaufkommen im Harz gegenüber dem im Solling deutlich. Durch die Jagdzeiten bilden sich zudem regelmäßig wiederkeh- 
rende Verteilungsmuster im Jahresverlauf ab. Der herausragende Peak für den Monat Juni und der tiefe Einschnitt im Juli finden hierin ihre Begründung. Auch die Hauptbejagungszeit des Rotwildes, von September bis Januar, spiegelt sich im Kurvenverlauf wider. Vereinzelte Streckenmeldungen aus den Monaten der Schonzeit ergeben sich hingegen in der Regel durch Fallwildfunde oder Hegeabschüsse.

Vergleicht man die Verläufe von Harz und Solling, fallen während des Hauptbejagungszeitraumes Unterschiede auf. So liegt der Spitzenwert des Streckenaufkommens in allen erfassten Jahren im Harz im Monat September, während er im Solling auf den Monat Oktober fällt. Daneben weist der Solling im Januar stets höhere Werte als im Dezember auf, während es im Harz eine kontinuierliche Abnahme der Werte von September bis Januar gibt. Bei der Betrachtung der Monatswerte hinsichtlich der Schwankungsbreite ihres jährlichen Aufkommens zeigt sich ein uneinheitliches Bild. In Abbildung 3.26 ist der jeweilige relative Variationskoeffizient des Mittelwerts eines jeden Monats abgetragen. Im Vergleich beider Untersuchungsgebiete fällt zunächst die generell höhere Variation der Monatswerte im Solling auf. Ausnahmen bilden lediglich die Monate Januar und Juni. Das Augenmerk soll auch hier auf den Hauptbejagungszeitraum gerichtet werden, also auf die Zeit von September bis Januar. In beiden Gebieten zeigen sich dort die niedrigsten Variationskoeffizientswerte im Jahresverlauf. Zugleich werden in dieser Periode mehr als $80 \%$ der Jahresstrecke beim Rotwild erzielt (vgl. Abb. 3.27). Beim Vergleich verschiedener Reviere hinsichtlich ihres Jagdstreckenaufkommens wird die Summe des zwischen September und Januar einer Periode er-

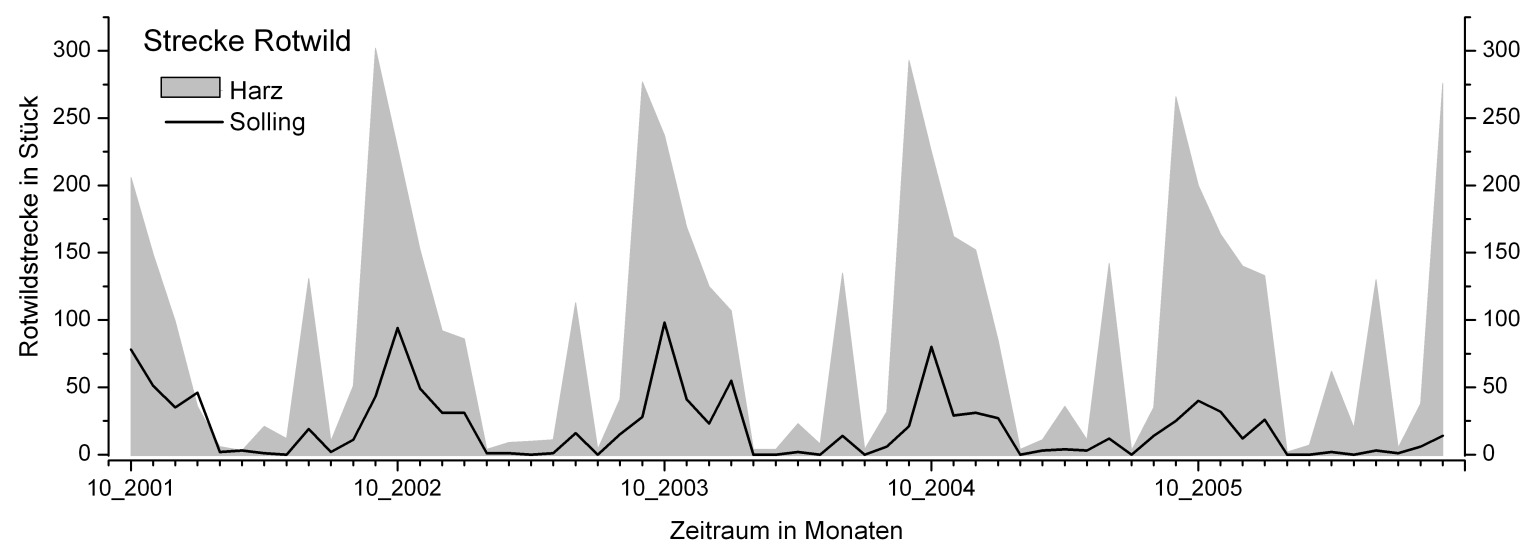

Abbildung 3.25: Jagdstreckenaufkommen und Fallwildmeldungen des Rotwildes im gesamten Untersuchungszeitraum, gesondert dargestellt für Harz und Solling; absolute Anzahl in Stück, zusammengefasst in Kalendermonaten 


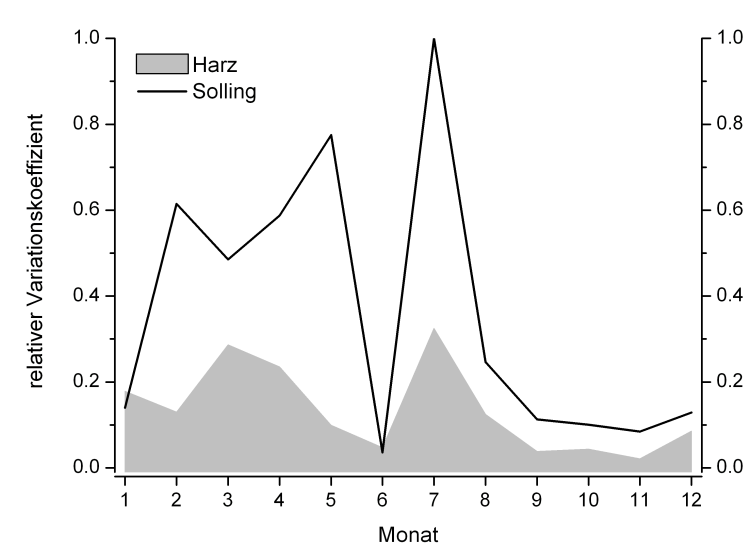

Abbildung 3.26: Relativer Variationskoeffizient $\left(V_{r}\right)$ der Monatsmittelwerte $(\bar{x})$ der Rotwildstrecke, errechnet für die Jahre 2002 bis 2005; getrennt dargestellt für Harz und Solling

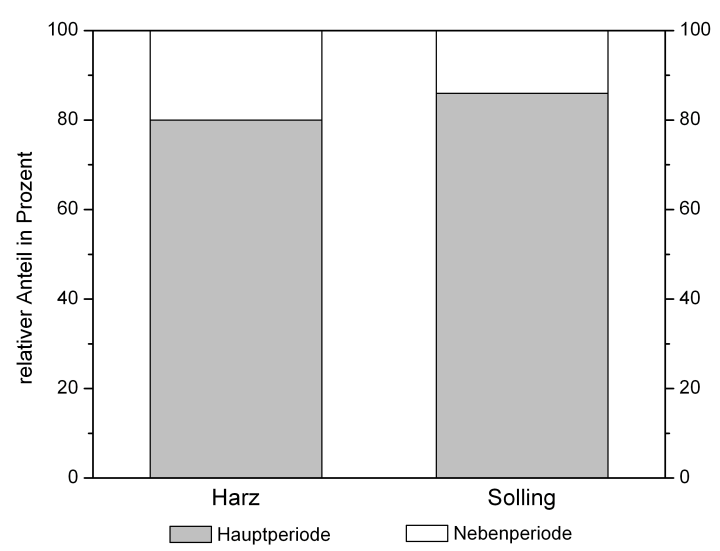

Abbildung 3.27: Anteil der Monatssummen aus Januar, September, Oktober, November und Dezember an der Gesamtjahresstrecke; bezeichnet als "Hauptperiode“

Tabelle 3.3: Mittlere relative Anteile ausgewählter Monate am Gesamtaufkommen der Rotwildjagdstrecken in Harz und Solling; berücksichtigter Zeitraum: 2002 bis 2005

\begin{tabular}{lrrrrrrrrrrr}
\hline Monat & \multicolumn{2}{c}{ Januar } & \multicolumn{3}{c}{ September } & \multicolumn{2}{c}{ Oktober } & \multicolumn{2}{c}{ November } & \multicolumn{2}{c}{ Dezember } \\
& $\bar{x}$ & $s$ & $\bar{x}$ & $s$ & $\bar{x}$ & $s$ & $\bar{x}$ & $s$ & $\bar{x}$ & $s$ \\
\hline Harz & 7,1 & 2,5 & 26,0 & 2,0 & 20,4 & 1,8 & 14,8 & 0,6 & 11,6 & 2,0 \\
Solling & 16,6 & 4,6 & 12,2 & 2,7 & 31,6 & 6,3 & 15,8 & 2,7 & 9,8 & 2,5 \\
\hline
\end{tabular}

$\bar{x}=$ arithmetisches Mittel (Angabe in Prozent), $s=$ Standardabweichung (Angabe in Prozentpunkten)

legten Rotwildes somit als ausreichend repräsentativ für das Gesamtjahresaufkommen dieser Wildart angesehen.

Darüber hinaus wird deutlich, dass die Streckenmeldungen des Monats September einen erheblichen Anteil dieser Summe stellen (siehe Monatsmittelwerte in Tab. 3.3). Dies betrifft in erster Linie die Werteberechnung im Harz, wo die Septemberjagdstrecke rund ein Drittel des Aufkommens des Hauptbejagungszeitraumes ausmacht. Wie jedoch unter 2.3.4 auf Seite 26 bereits geschildert wurde, konnte die räumliche Zuordnung jener Jagdstreckendaten, die vor dem 01.10.2001 registriert worden sind, nicht erfolgen. Bei der Berechnung der jährlichen Perioden würden die Septemberwerte im Jahr 2001 also fehlen. Hieraus ergäbe sich ein Problem, denn eine Beschränkung aller Jahressummen auf die Daten von Oktober bis Januar, nur um für alle Jahre vergleichbare Werte zu erhalten, hätte vermutlich einen hohen Informationsverlust zur Folge. 
Im Sinne der rein periodischen Aufkommensberechnung für die Reviere erscheint darum die Rückrechnung der Septemberwerte für das Jahr 2001 als vertretbare Lösung. Ausgehend vom Mittel der Jahre 2002 bis 2006 kann für den September ein Anteil von $36 \%$ des gemeinsamen Streckenaufkommens der Monate September bis Dezember zugrunde gelegt werden (im Solling von 18 \%). Dieser Wert wird im Folgenden als Berechnungsgrundlage der mutmaßlichen Streckenhöhe im September 2001 angenommen und die Daten hiermit entsprechend ergänzt.

\subsubsection{Räumliche Verteilung der Rotwilderlegungen}

Die Höhe der jährlichen Rotwildstrecke wurde entsprechend der unter 2.3.4 auf Seite 26 beschriebenen Methode auf Revierebene berechnet. Eine erste Übersicht zu den mittleren Werten der Reviere in Harz und Solling bietet Tabelle 3.4. Die dort aufgelisteten Mittelwerte jährlich erlegten Rotwildes beziehen sich auf den Hauptbejagungszeitraum (vgl. 3.3.1) und lassen sich somit nicht direkt mit den jagdlich üblichen Streckenquotienten vergleichen, da nicht die gesamte Stückzahl erlegten Rotwildes je 100 Hektar hierfür zugrunde liegt. Für die Tabelle sind die Werte über alle Forstamtsgrenzen innerhalb eines Gebietes hinweg gemittelt, jedoch nach Jahren getrennt worden.

Die mittleren Streckenaufkommen sind im Harz insgesamt höher als im Solling (Mittelwert Harz $=1,2$ zu 0,6 im Solling bei $p<0,001)$. Innerhalb des Harzes, dessen mittlere Streckenquotienten zwischen 1,11 und 1,31 Stück Rotwild je 100 Hektar liegen, ergeben sich keine signifikanten Unterschiede zwischen den untersuchten Jahren. Lediglich im Solling fällt der Mittelwert der Periode 2005/06 signifikant geringer aus als die Mittelwerte der beiden Perioden zuvor. Der Median liegt, mit einer Ausnahme, in allen Fällen deutlich niedriger als das arithmetische Mittel. Es handelt sich also um eine linksschiefe Verteilung durch einige wenige Extremwerte von Revieren mit besonders hohen Jagdstreckendaten. Die in Tabelle 3.4 ebenfalls angegebenen relativen Variationskoeffizienten vermitteln darüber hinaus einen Eindruck von der Verschiedenheit der Gebiete hinsichtlich der Varianz ihrer Revierdaten. So erweist sich der Solling diesbezüglich in allen Jahren als das deutlich differenziertere Gebiet. Die Unterschiede zwischen den Revieren hinsichtlich der Höhe ihrer jeweiligen Rotwildstrecke sind dort also stets ausgeprägter als im Harz.

Was in der Tabelle 3.4 nur in Form von Mittel- und Varianzwerten ausgedrückt wurde, soll in den Abbildungen 3.28 und 3.29 durch eine sechsstufige Klassifizierung weiter veranschaulicht werden. Getrennt für Harz und Solling sind die Anteile der Reviere 
3. Charakterisierung der Stichprobenpunkte

Tabelle 3.4: Jährliches mittleres Rotwildstreckenaufkommen in Stück je 100 ha im Zeitraum September bis Januar, erhoben auf Revierebene und gemittelt für das jeweilige Gebiet

\begin{tabular}{lccrrrrr}
\hline Gebiet & Periode & $n$ & $\bar{x}$ & $s$ & $V$ & $V_{r}$ & $Z$ \\
\hline \multirow{2}{*}{ Harz } & $2001 / 02$ & 49 & 1,11 & 0,88 & 0,79 & $11,36 \%$ & 0,82 \\
& $2003 / 04$ & 49 & 1,31 & 0,89 & 0,68 & $9,66 \%$ & 1,40 \\
& $2005 / 06$ & 49 & 1,29 & 0,79 & 0,61 & $8,77 \%$ & 1,22 \\
\multirow{5}{*}{ Solling } & $2001 / 02$ & 27 & 0,72 & 0,63 & 0,87 & $16,82 \%$ & 0,69 \\
& $2002 / 03$ & 27 & 0,71 & 0,58 & 0,82 & $15,71 \%$ & 0,65 \\
& $2005 / 06$ & 27 & 0,38 & 0,27 & 0,98 & $18,93 \%$ & 0,23 \\
\hline
\end{tabular}

$n=$ Anzahl der Reviere, $\bar{x}=$ arithmetisches Mittel der Rotwilderlegungen, $s=$ Standardabweichung, $V=$ Variationskoeffizient, $V_{r}=$ relativer Variationskoeffizient, $Z=$ Median

verschieden hoher Rotwildindizes in den jeweils relevanten Perioden aufgeführt. Die Klassengrenzen wurden hierzu in 0,5-Schritten gesetzt, wobei die erste Klasse einen Indexwert von 0 repräsentiert und die letzte Klasse alle Werte von 2,0 bis 3,93 einschließt. Im Harz (Abb. 3.28) ist der Anteil der Nullklasse sehr gering und bleibt in allen drei Jahren unter $10 \%$. Die übrigen Klassen weisen eine annähernd gleiche Verteilung auf, wobei die höheren Anteile der niedrigeren Klassen in Periode 2001/02 leicht herausstehen. Im Solling (Abb. 3.29) zeigt sich eine ungleichmäßigere Verteilung. Der Anteil

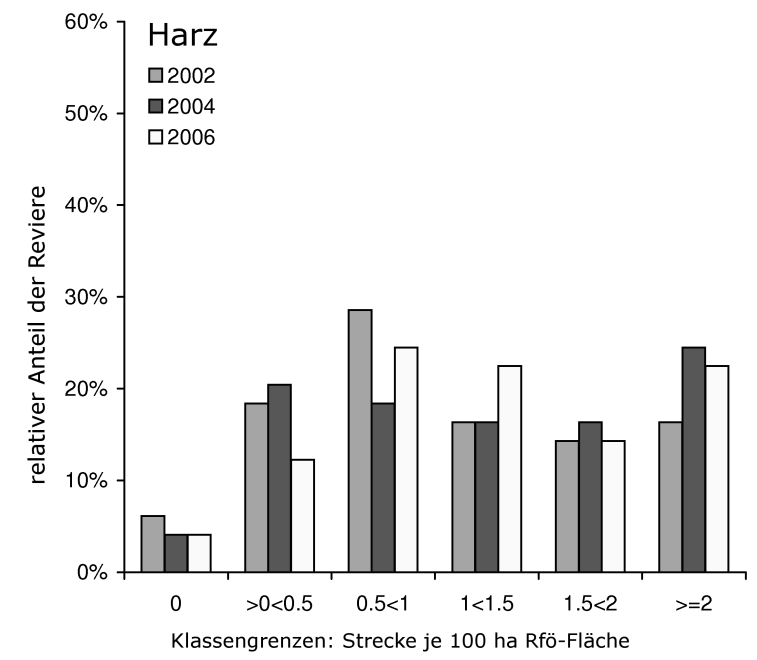

Abbildung 3.28: Relativer Anteil der Reviere an Streckenklassen im Harz, klassifiziert nach Rotwildstrecke je 100 ha; jeweiliger Zeitraum: September bis Januar; $n=49$

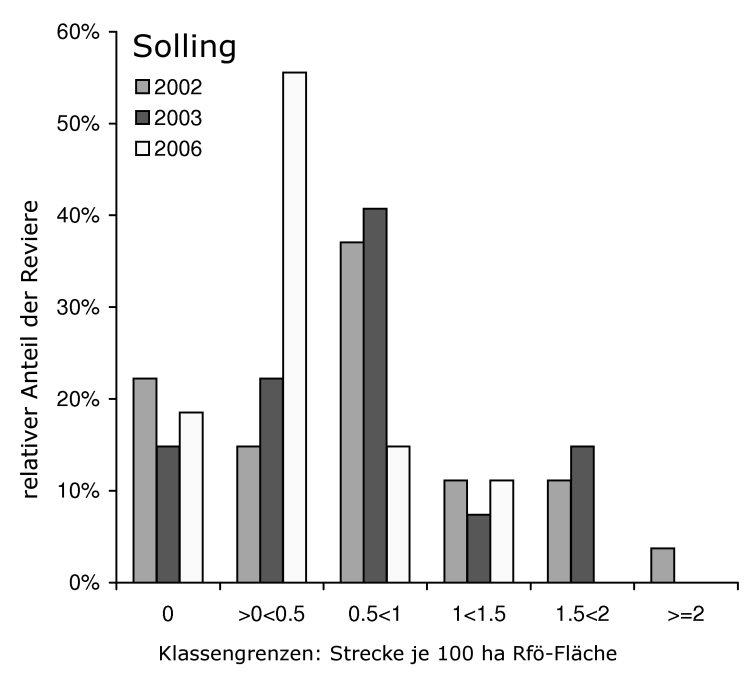

Abbildung 3.29: Relativer Anteil der Reviere an Streckenklassen im Solling, klassifiziert nach Rotwildstrecke je 100 ha; jeweiliger Zeitraum: September bis Januar; $n=27$ 
der Nullklasse liegt in allen Jahren über $15 \%$. In der Periode 2001/02 fällt sogar jedes fünfte Revier in diese Kategorie. Die deutlich größten Anteile aber stellen Reviere mit Werten zwischen $>0<0,5$ und $0,5<1$. Im Jahr 2005/06 ist die Klasse 1,5<2 bereits nicht mehr mit Werten besetzt und Reviere mit einem Rotwildindex von 2,0 oder größer kommen nur in Periode 2001/02 vor.

\subsubsection{Beanspruchte Jagdfläche je Revier}

Der zeitliche Aufwand zum Erreichen der Rotwildstrecke ist nicht durch die vorhandenen Erlegungsdaten greifbar. Somit können keine Aussagen zur Präsenz des jagenden Menschen in einem Revier getroffen werden. Um die Art der Bejagung trotzdem als Differenzierungsmerkmal zwischen den Revieren heranziehen zu können, soll das Potential der erfassten Streckendaten weitestgehend ausgeschöpft werden. Hierzu wurden die Abteilungen der Reviere, in denen Rotwildabschüsse getätigt wurden, ins Verhältnis zur Gesamtzahl der Abteilungen des jeweiligen Revieres gesetzt. Im Ergebnis zeigt sich eine lineare Zunahme des Flächenanteils $(r=0,94282)$ mit Erhöhung der Abschusszahl (Abb. 3.30). Im Bereich geringen Streckenaufkommens ist die Varianz der dazugehörigen Flächenwerte naturgemäß gering. Im Bereich höherer Abschusszahlen zeigen sich jedoch einige Unterschiede zwischen den Revieren.

Betrachtet man die Anzahl der am Streckenergebnis beteiligten Abteilungen eines Revieres und teilt die Anzahl des dort erlegten Rotwildes durch diese Zahl, ergibt

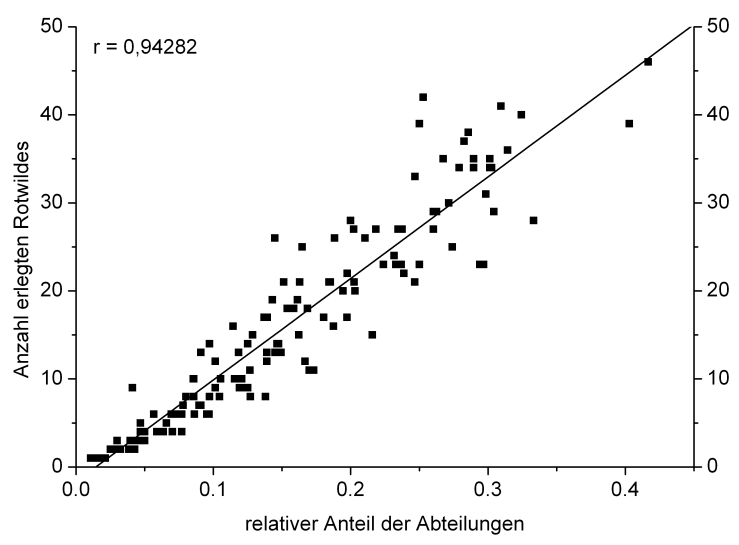

Abbildung 3.30: Relativer Anteil der Abteilungen mit Erlegungen an der Gesamtzahl der Abteilungen eines Revieres; gemeinsam dargestellt für Harz und Solling aller Perioden

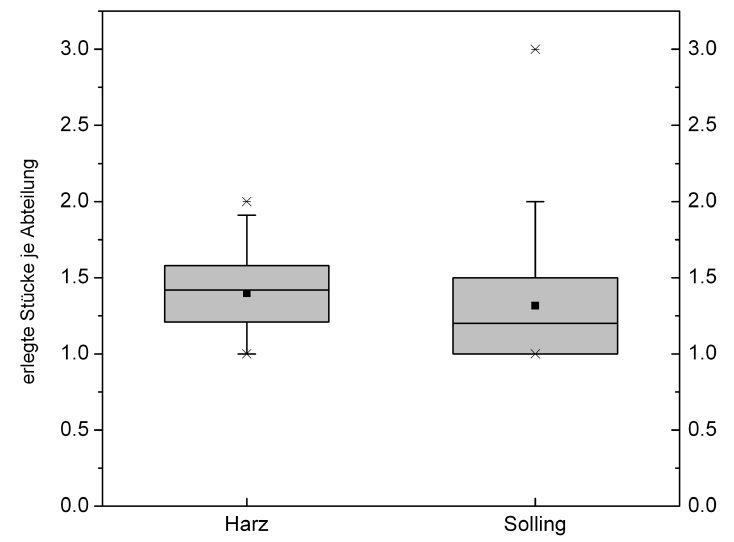

Abbildung 3.31: Mittlere Anzahl der Erlegungen je Abteilung; ausschließlich errechnet für Abteilungen mit Erlegung, getrennt für Harz und Solling über alle Perioden 
sich für das Gebiet des Harzes ein Wert von 1,4 Stück Rotwild je Abteilung ( $s=$ 0,25). Im Solling wird ein Wert von 1,3 Stück Rotwild je Abteilung erreicht $(s=$ 0,41). Vergleicht man die Mittelwerte der beiden Gebiete miteinander (Abb. 3.31), erweist sich die Abweichung von 0,08 als nicht signifikant $(p=0,213)$. Der relative Variationskoeffizient liegt mit 4,6 \% im Solling jedoch deutlich über dem im Harz mit $1,9 \%$. 


\section{Analyse des Schälschadenaufkommens}

\subsection{Aktuelle und vorangegangene Schälschäden}

\subsubsection{Neuschälaufkommen der Inventuren}

Zur Berechnung der mittleren Winterneuschäle an Fichte werden die Daten zunächst nach Untersuchungsgebiet unterschieden. Eine weitere Gliederung erfolgt anhand der einzelnen Jahre, in denen die dortigen drei Inventuren durchgeführt wurden. Ein geeigneter Lageparameter für die weder im Solling noch im Harz normalverteilte Zielvariable (Shapiro-Wilk-Test jeweils $p<0,001$; vgl. auch Abb. 4.1) wäre der Median, denn die Klassifizierung der aufgenommenen Bäume erfolgt ja stets in „geschält" oder „ungeschält", so dass nur ganze Zahlen vorkommen. Wie sich bereits anhand eines ersten Histogrammes zur Häufigkeitsverteilung ableiten lässt (Abb. 4.1), liegt der Median allerdings in beiden Fällen beim Wert Null, sodass stattdessen auf das arithmetische Mittel zurückgegriffen werden soll. Getrennt nach den jeweiligen Inventurjahren sind die Ergebnisse dieser Berechnungen in Tabelle 4.1 aufgeführt. Der zugehörige Standardfehler wurde, mangels Normalverteilung der Daten, mit Hilfe der Bootstrap-Methode ermittelt. Hierzu wurde 1.000 mal eine Zufallsstichprobe in Höhe des tatsächlichen Stichprobenumfanges (mit Zurücklegen) gezogen und jedes Mal der Mittelwert berechnet. Durch diese Resampling-Methode ergab sich in Summe eine annähernde Normalverteilung der Werte, so dass hieraus der Standardfehler bestimmt werden konnte.

Für den Solling errechnet sich für das Jahr 2002 ein mittleres Schälprozent von 3,99 \%. Nach einem geringfügig darunter liegenden Wert von 3,11 \% im Jahr 2003 ergibt sich für das Jahr 2006 schließlich ein Neuschälprozent von 2,06 \%. Statistisch signifikant hebt sich hierbei nur der Mittelwert des Jahres 2006 von dem des Jahres 2003 (mittlere Differenz: 1,056 Prozentpunkte) sowie von dem des Jahres 2002 (mittlere Differenz: 1,934 Prozentpunkte) ab. Im Harz liegen die mittleren Schälprozente in allen drei Inventurjahren bei knapp unter $5 \%$ und weisen in ihrer Höhe keine si- 


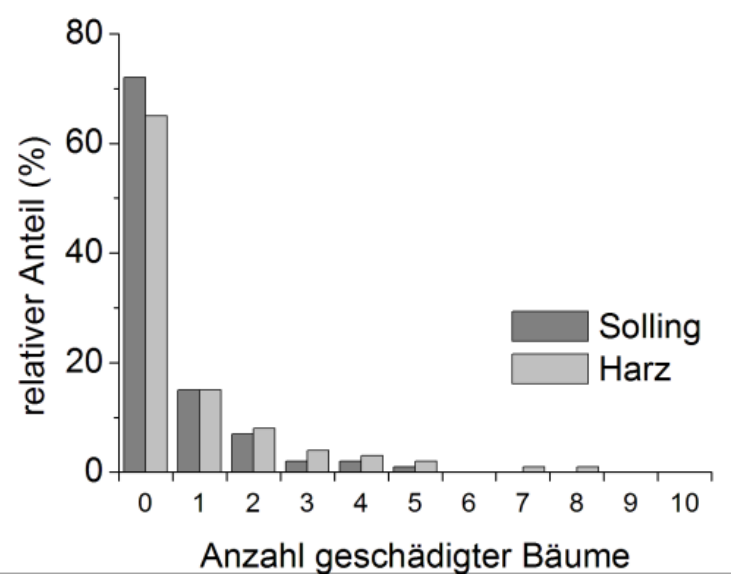

Abbildung 4.1: Häufigkeit von Winterneuschäle, klassifiziert für Solling und Harz

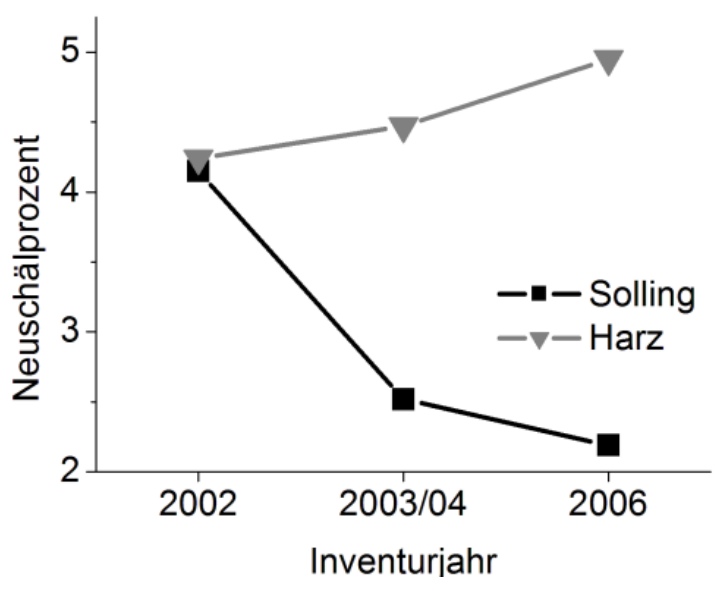

Abbildung 4.2: Mittlere Winterneuschälprozente in Solling und Harz, getrennt nach Inventurjahren

gnifikanten Unterschiede zueinander auf (vgl. hierzu auch Abb. 4.2). Als statistisch gesichertes Signifikanzniveau wird hier, wie auch bei allen folgenden Ergebnissen, stets ein Wert von $p<0,05$ angenommen. Stellt man die Mittelwerte beider Untersuchungsgebiete gegeneinander, stehen für diesen Vergleich ausschließlich die Jahre 2002 und 2006 zur Verfügung, da nur in diesen Jahren in beiden Gebieten zugleich eine Inventur durchgeführt wurde. Auch hier liegt lediglich im Jahr 2006 das Neuschälprozent aus dem Solling um 2,9 Prozentpunkte statistisch signifikant unter dem Vergleichswert von 4,94 \% im Harz. Im Inventurjahr 2002 hingegen hebt sich das Neuschälprozent im

Tabelle 4.1: Mittleres Winterneuschälprozent an Fichte in Harz und Solling der Jahre 2002, 2003, 2004 und 2006

\begin{tabular}{llrrrrrr}
\hline Gebiet & $J a h r$ & $n$ & $\bar{x}$ & $s$ & $V$ & $V_{r}$ & $s_{\bar{x}}$ \\
\hline \multirow{2}{*}{ Solling } & 2002 & 749 & $3,99 \%$ & 10,33 & 2,59 & $9,46 \%$ & 0,38 \\
& 2003 & 758 & $3,11 \%$ & 10,49 & 3,37 & $12,25 \%$ & 0,38 \\
& 2006 & 751 & $2,06 \%$ & 7,76 & 3,77 & $13,75 \%$ & 0,28 \\
\multirow{4}{*}{ Harz } & 2002 & 456 & $4,57 \%$ & 11,19 & 2,45 & $11,47 \%$ & 0,52 \\
& 2004 & 611 & $4,91 \%$ & 12,27 & 2,50 & $10,11 \%$ & 0,50 \\
& 2006 & 638 & $4,94 \%$ & 12,42 & 2,51 & $9,95 \%$ & 0,49 \\
\hline
\end{tabular}

$n=$ Anzahl, $\bar{x}=$ arithmetisches Mittel, $s=$ Standardabweichung, $V=$ Variationskoeffizient $V_{r}=$ relativer Variationskoeffizient, $s_{\bar{x}}=$ Standardfehler von $\bar{x}$ 
Tabelle 4.2: Mittleres Winterneuschälprozent an Fichte in Harz und Solling der Jahre 2002, 2003, 2004 und 2006, unterschieden zwischen homogen und heterogen besetzten Plots

\begin{tabular}{lllrrrrrr}
\hline Jahr & Gebiet & Datenset & $n$ & $\bar{x}$ & $s$ & $V$ & $V_{r}$ & $s_{\bar{x}}$ \\
\hline \multirow{2}{*}{2002} & Solling & homogen & 313 & $4,05 \%$ & 7,84 & 1,94 & $10,94 \%$ & 0,44 \\
& Solling & heterogen & 436 & $3,95 \%$ & 11,80 & 2,99 & $14,31 \%$ & 0,57 \\
& Harz & homogen & 209 & $4,01 \%$ & 7,27 & 1,81 & $12,54 \%$ & 0,50 \\
& Harz & heterogen & 247 & $5,05 \%$ & 13,66 & 2,70 & $17,21 \%$ & 0,87 \\
2003 & Solling & homogen & 310 & $2,51 \%$ & 5,59 & 2,23 & $12,65 \%$ & 0,32 \\
& Solling & heterogen & 448 & $3,53 \%$ & 12,82 & 3,63 & $17,16 \%$ & 0,61 \\
2004 & Harz & homogen & 285 & $4,21 \%$ & 8,14 & 1,93 & $11,45 \%$ & 0,48 \\
& Harz & heterogen & 326 & $5,52 \%$ & 14,96 & 2,71 & $15,01 \%$ & 0,83 \\
\multirow{2}{*}{2006} & Solling & homogen & 271 & $2,17 \%$ & 5,00 & 2,30 & $14,00 \%$ & 0,30 \\
& Solling & heterogen & 480 & $1,99 \%$ & 8,95 & 4,50 & $20,53 \%$ & 0,41 \\
& Harz & homogen & 279 & $4,48 \%$ & 9,18 & 2,05 & $12,27 \%$ & 0,55 \\
& Harz & heterogen & 359 & $5,29 \%$ & 14,44 & 2,73 & $14,41 \%$ & 0,76 \\
\hline
\end{tabular}

$n=$ Anzahl, $\bar{x}=$ arithmetisches Mittel, $s=$ Standardabweichung, $V=$ Variationskoeffizient , $V_{r}=$ relativer Variationskoeffizient, $s_{\bar{x}}=$ Standardfehler

Solling, mit einer Differenz von 0,6 Prozentpunkten, nicht signifikant $(p=0,102)$ vom Neuschälprozent desselben Jahres im Harz ab.

Im Methodenteil wurde zwischen homogenen und heterogenen Stichprobenpunkten unterschieden (siehe 2.2.2, Seite 15). In Tabelle 4.2 sind diese nun mit ihren jeweiligen Schälprozenten aufgeführt. Um auch Mittelwertabweichungen dieser Datensets weiter zu qualifizieren, wurden alle sich ergebenden Kombinationen, die nach Berücksichtigung des Jahres und der Art der Stichprobe vergleichbar waren, in ihren Mittelwerten gegeneinander getestet (zum Beispiel heterogene Datensets beider Gebiete innerhalb desselben Jahres zueinander). Zur vorangegangenen Gesamtbetrachtung (Tab. 4.1) ergeben sich hierdurch jedoch keine zusätzlichen Auffälligkeiten. So zeigen sich für den Solling im Vergleich zum Harz - auch bei der Unterscheidung homogener und heterogener Datensets erneut nur für das Jahr 2006 statistisch signifikant niedrigere Werte. Lediglich hinsichtlich der Höhe der Mittelwertdifferenzen lässt sich für die heterogene Stichprobe mit 3,3 Prozentpunkten eine größere Abweichung als bei der homogenen Stichprobe (2,3 Prozentpunkte) feststellen. Alle anderen Unterschiede in der Höhe der jährlichen mittleren Schälprozente erweisen sich weder im Vergleich der Untersuchungsgebiete 
Tabelle 4.3: Mittleres Neuschälprozent an Fichte in Harz und Solling im Jahr 2006, bezogen auf Klassen vorangegangenen Neuschälvorkommens; berechnet über sämtliche Stichprobenpunkte mit Fichtenvorkommen; $n=446 / 627$ (Harz/Solling)

\begin{tabular}{llcrrrrrr}
\hline Gebiet & Sig. & Klasse & $n$ & Anteil & $\bar{x}$ & $s$ & $V$ & $V_{r}$ \\
\hline \multirow{2}{*}{ Harz } & 0,022 & 0 & 247 & $55 \%$ & $4,16 \%$ & 11,80 & 2,84 & $18,06 \%$ \\
& & 1 & 138 & $31 \%$ & $6,36 \%$ & 12,22 & 1,92 & $16,35 \%$ \\
& & 2 & 61 & $14 \%$ & $8,61 \%$ & 13,33 & 1,55 & $19,82 \%$ \\
\multirow{3}{*}{ Solling } & 0,855 & 0 & 402 & $64 \%$ & $2,06 \%$ & 8,89 & 4,33 & $21,57 \%$ \\
& & 1 & 164 & $26 \%$ & $2,44 \%$ & 7,60 & 3,11 & $24,28 \%$ \\
& & 2 & 61 & $10 \%$ & $2,43 \%$ & 4,87 & 2,01 & $25,69 \%$ \\
\hline
\end{tabular}

Sig. $=p-$ Wert,$n=$ Anzahl, Anteil $=$ relativer Anteil der Klasse am Datenset, $\bar{x}=$ arithmetisches Mittel der Neuschäle, $s=$ Standardabweichung, $V=$ Variationskoeffizient, $V_{r}=$ relativer Variationskoeffizient

zueinander noch im Vergleich heterogener und homogener Datensets innerhalb eines Gebietes als signifikant.

\subsubsection{Neuschäle an Orten mit Altschälvorkommen}

Wie unter 2.3.1 auf Seite 21 beschrieben, wird die im Zuge der Inventur erfasste Altschäle nicht unmittelbar als Parameter herangezogen. Stattdessen handelt es sich um das dokumentierte Neuschälaufkommen vorangegangener Perioden, welches zur Einstufung eines Stichprobenpunktes in eine von drei Klassen führt. In Tabelle 4.3 sind diese Klassen der Vorschädigungsquote $(2=$ in zwei von zwei Inventurjahren wurde hier Neuschäle erfasst) für die beiden Untersuchungsgebiete aufgeführt. Da ja nur die Daten des Jahres 2006 getestet werden können, beschränkt sich der Datenpool auf insgesamt 1.073 Punkte, an denen zudem in allen drei Inventurjahren eine Aufnahme stattfand.

Im Harz zeigt die Varianzanalyse insgesamt signifikante Unterschiede zwischen den verschiedenen Klassenwerten. Das mittlere Neuschälprozent der Klasse 0, also der Stichprobenpunkte, an denen weder im Jahr 2004 noch im Jahr 2002 ein Neuschälschaden an Fichte dokumentiert worden war, liegt bei 4,2 \%. Dieser Wert bleibt deutlich unter denen der Klassen mit vorangegangener Schäle (1 und 2), welche im Mittel ein um $70 \%$ höheres Neuschälprozent aufweisen $(\bar{x}=7,1 \% ; p=0,013)$. In der Klasse 2 erhöht sich das Neuschälprozent noch einmal um rund ein Viertel gegenüber der Klasse 1. Mit 8,6 \% mittlerer Neuschäle im Jahr 2006 liegt dieser Wert signifikant über dem der Klas- 
sen 0 und $1(\bar{x}=5 \% ; p=0,029)$. Die geringen Mittelwertabweichungen der einzelnen Klassen im Solling sind hingegen nicht signifikant. Neben den leicht höheren Werten des relativen Variationskoeffizienten gegenüber denen des Harzes fällt im Solling vor allem der höhere Anteil der Klasse 0 auf. So wurde an 64 \% der hier betrachteten Stichprobenpunkte in keiner der vorangegangenen Inventuren ein Neuschälschaden festgestellt, während diese Klasse im Harz nur $55 \%$ der Stichprobenpunkte einschließt. Enstsprechend höher fallen im Harz die Klassenanteile der ein- bis zweimal zuvor mit Neuschäle dokumentierten Punkte aus.

Als weitere Untersuchung schließt sich eine Klassifizierung des Neuschälaufkommens der Inventur 2006 an, in die die zu jenem Zeitpunkt am Stichprobenpunkt erfasste Altschäle als Prozentwert eingeht. Da eine differenziertere Analyse der Altschälwerte nicht einwandfrei möglich ist (vergleiche 2.3.1, S. 20), werden hierfür wieder nur die Inventurdaten des Jahres 2006 zugrunde gelegt. Überschneidungen und Doppelungen von Altschälwerten verschiedener Perioden sollen so vermieden werden. Der Datenpool fällt mit 1.076 Stichprobenpunkten trotzdem etwas höher als bei der vorangegangenen Untersuchung über zurückliegende Neuschälereignisse aus, da in dem vorliegenden Fall keine Kontinuität der Datenaufnahme über alle drei Inventurjahre an einem Punkt erforderlich ist. Die Berechnung der Altschälprozente bezieht sich, wie auch die der Neuschäle, ausschließlich auf die Baumart Fichte. Die Einteilung der Werte erfolgte in jeweils sechs Klassen, deren Grenzen für beide Gebiete identisch gewählt wurden. In Tabelle 4.4 sind die Ergebnisse detailliert dargestellt.

Erneut zeigen sich für den Harz deutliche Unterschiede der mittleren Schälprozente, die mit zunehmendem Altschälprozent der jeweiligen Klasse ansteigen. Wie schon bei der Betrachtung der Neuschäle vorangegangener Perioden (Tab. 4.3) können die Mittelwertabweichungen insgesamt als statistisch signifikant gewertet werden. Interessant ist, dass die tiefsten und höchsten Neuschälwerte jeweils nicht in den extremen Klassen der Altschäle vorkommen (also für $0 \%$ oder $100 \%$ Altschälvorkommen), sondern in der jeweils darüber bzw. darunter liegenden. Allerdings weisen die niedrigste und die höchste Klasse auch die wenigsten Argumente auf und liegen in ihrer Varianz entsprechend hoch. Mit einem Neuschälprozent von 2,2 \% setzt sich der Mittelwert aus erster und zweiter Klasse, also 0 bis $24 \%$ vorhandener Altschäle, dennoch hochsignifikant $(p<0,001)$ vom Rest mit einem Mittelwert von 6,2 \% ab. Ebenso klar liegt das Neuschälprozent von Stichprobenpunkten mit $75 \%$ bis $100 \%$ umfassendem Altschälanteil über dem der restlichen Punkte. Hier steht dem gemeinsamen Durch- 
Tabelle 4.4: Mittleres Neuschälprozent an Fichte in Harz und Solling im Jahr 2006, bezogen auf Klassen dokumentierter Altschälschäden; berechnet über sämtliche Stichprobenpunkte mit Fichtenvorkommen; $n=638 / 751$ (Harz/Solling)

\begin{tabular}{|c|c|c|c|c|c|c|c|c|}
\hline Gebiet & Sig. & Klasse & $n$ & Anteil & $\bar{x}$ & $s$ & $V$ & $\overline{V_{r}}$ \\
\hline \multirow[t]{6}{*}{ Harz } & 0,001 & $0 \%$ & 91 & $14 \%$ & $3,21 \%$ & 15,79 & 4,93 & $51,64 \%$ \\
\hline & & $1-24 \%$ & 103 & $16 \%$ & $1,25 \%$ & 3,56 & 2,86 & $28,15 \%$ \\
\hline & & $25-49 \%$ & 110 & $17 \%$ & $3,79 \%$ & 8,38 & 2,21 & $21,10 \%$ \\
\hline & & $50-74 \%$ & 122 & $19 \%$ & $5,10 \%$ & 11,21 & 2,20 & $19,88 \%$ \\
\hline & & $75-99 \%$ & 134 & $21 \%$ & $8,56 \%$ & 14,57 & 1,70 & $14,71 \%$ \\
\hline & & $100 \%$ & 78 & $12 \%$ & $6,97 \%$ & 15,91 & 2,28 & $25,84 \%$ \\
\hline \multirow[t]{6}{*}{ Solling } & 0,868 & $0 \%$ & 186 & $25 \%$ & $1,60 \%$ & 9,50 & 5,96 & $43,68 \%$ \\
\hline & & $1-24 \%$ & 80 & $11 \%$ & $1,59 \%$ & 5,43 & 3,42 & $38,29 \%$ \\
\hline & & $25-49 \%$ & 105 & $14 \%$ & $2,70 \%$ & 5,78 & 2,14 & $20,85 \%$ \\
\hline & & $50-74 \%$ & 169 & $23 \%$ & $2,17 \%$ & 5,18 & 2,39 & $18,38 \%$ \\
\hline & & $75-99 \%$ & 135 & $18 \%$ & $2,15 \%$ & 7,58 & 3,53 & $30,34 \%$ \\
\hline & & $100 \%$ & 76 & $10 \%$ & $2,38 \%$ & 11,74 & 4,94 & $56,68 \%$ \\
\hline
\end{tabular}

Sig. $=p-$ Wert,$n=$ Anzahl, Anteil $=$ relativer Anteil der Klasse am Datenset, $\bar{x}=$ arithmetisches Mittel der Neuschäle, $s=$ Standardabweichung, $V=$ Variationskoeffizient, $V_{r}=$ relativer Variationskoeffizient

schnitt von 3,4 \% der niedrigeren Klassen ein mittleres Neuschälprozent von 8,0 \% gegenüber. Somit weisen Stichprobenpunkte mit mindestens $75 \%$ Altschäle im Mittel ein um rund $130 \%$ erhöhtes Neuschälvorkommen auf $(p<0,001)$. Der relative Variationskoeffizient der Klasse mit $75 \%$ bis $99 \%$ Altschäle ist zudem der niedrigste Wert innerhalb des Datensets und unterstreicht die Regelmäßigkeit hoher Neuschälprozente in diesem Bereich.

Die Werte des Sollings zeigen erneut keinen eindeutigen Trend. Zwar liegen die Neuschälprozente der niedrigen Altschälklassen auch bei dieser Betrachtung unterhalb der darauffolgenden, doch sind diese Unterschiede statistisch nicht signifikant. Insgesamt liegen die Mittelwerte viel enger beieinander als im Harz. Gleichzeitig sind die Varianzen innerhalb der einzelnen Klassen in beiden Gebieten auf vergleichbarem Niveau. An der schwachen Informationslage bei Betrachtung dreier Klassen, wie zuvor in Tabelle 4.3 , ändert sich also auch bei weiterer Differenzierung in sechs Klassen und dem Bezug auf Altschälprozente nichts. 
Tabelle 4.5: Mittleres Neuschälprozent an Fichte in Harz und Solling, bezogen auf Klassen der Höhenlage ü.NN; berechnet über sämtliche Stichprobenpunkte mit Fichtenvorkommen und alle drei Inventurjahre; $n=1.705 / 2.258$ (Harz/Solling)

\begin{tabular}{llcrrrrr}
\hline Gebiet & Sig. & Höhenklasse ü.NN & $n$ & $\bar{x}$ & $s$ & $V$ & $V_{r}$ \\
\hline \multirow{2}{*}{ Harz } & 0,001 & $236-299 \mathrm{~m}$ & 30 & $0,74 \%$ & 4,06 & 5,48 & $100,00 \%$ \\
& & $300-399 \mathrm{~m}$ & 135 & $4,84 \%$ & 13,57 & 2,80 & $24,13 \%$ \\
& & $400-499 \mathrm{~m}$ & 343 & $6,21 \%$ & 14,27 & 2,30 & $12,40 \%$ \\
& $500-599 \mathrm{~m}$ & 678 & $5,06 \%$ & 12,65 & 2,50 & $9,61 \%$ \\
& & $600-699 \mathrm{~m}$ & 290 & $5,22 \%$ & 11,14 & 2,13 & $12,52 \%$ \\
& & $700-799 \mathrm{~m}$ & 169 & $1,73 \%$ & 4,00 & 2,31 & $17,75 \%$ \\
\multirow{5}{*}{ Solling } & & $800-894 \mathrm{~m}$ & 60 & $3,21 \%$ & 6,29 & 1,96 & $25,33 \%$ \\
& 0,126 & $145-239 \mathrm{~m}$ & 73 & $1,02 \%$ & 4,53 & 4,44 & $51,95 \%$ \\
& & $240-339 \mathrm{~m}$ & 446 & $3,74 \%$ & 12,50 & 3,34 & $15,81 \%$ \\
& $340-439 \mathrm{~m}$ & 1194 & $2,98 \%$ & 8,60 & 2,89 & $8,36 \%$ \\
& $440-525 \mathrm{~m}$ & 545 & $2,92 \%$ & 9,58 & 3,28 & $14,05 \%$ \\
\hline
\end{tabular}

Sig. $=p$-Wert, $n=$ Anzahl der Stichprobenpunkte, $\bar{x}=$ arithmetisches Mittel der Neuschäle, $s=$ Standardabweichung, $V=$ Variationskoeffizient, $V_{r}=$ relativer Variationskoeffizient

\subsection{Schälaufkommen verschiedener Geländeformen}

\subsubsection{Differenzierung nach Höhenlage}

Getrennt nach Untersuchungsgebiet sind die verschiedenen Höhenlagen in Klassen eingeteilt worden und mit den zugehörigen Werten in Tabelle 4.5 aufgeführt. Die Klassengrenzen sind in 100-Meter-Schritten eingestellt. Entsprechend dem Stichprobenvorkommen reicht die Skala im Harz von 236 bis 894 Meter über Normalnull und führt somit zu einer tatsächlich geringeren Spreite in der ersten und geringfügig auch in der letzten der insgesamt sieben Klassen. Die Anzahl der Argumente je Klasse ist uneinheitlich. In der Höhenlage zwischen 500 und 599 m wird mit 678 Punkten der höchste Klassenwert erreicht. In die Klasse 236 bis $299 \mathrm{~m}$ fallen hingegen nur 30 Stichprobenpunkte. Die zugehörigen relativen Variationskoeffizienten nehmen dort entsprechende Extremwerte an.

Den höchsten Wert der Neuschäle nimmt die Höhenklasse 400 bis 499 m an (vergl. auch Abb. 4.3). Innerhalb der dortigen Stichprobenpunkte weisen im Mittel 6,21 \% der vorhandenen Fichten im Winter frische Schälschäden auf. Das dortige Schälprozent liegt damit signifikant $(p=0,038)$ über dem Niveau von $4,48 \%$ der restlichen Stich- 


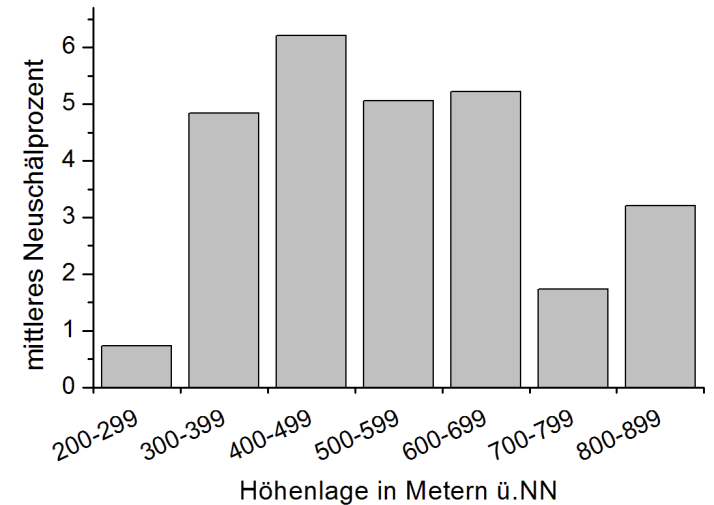

Abbildung 4.3: Mittleres Neuschälprozent der Inventurpunkte je Höhenklasse im Harz

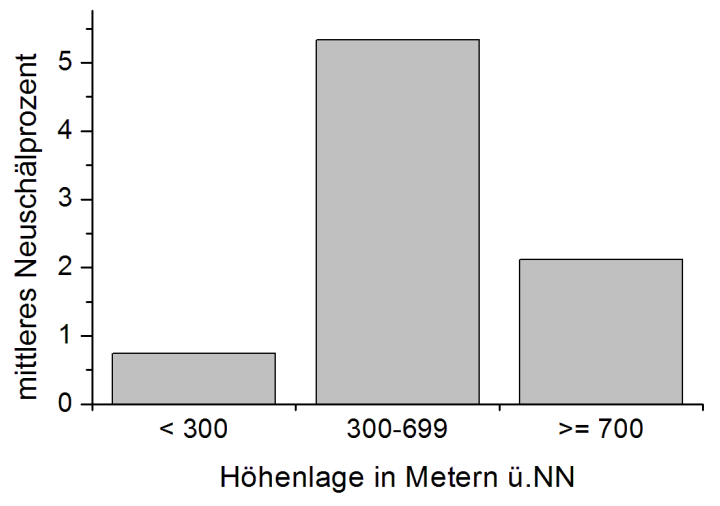

Abbildung 4.4: Mittleres Neuschälprozent der Inventurpunkte für niedrige, mittlere und hohe Lagen im Harz

probenpunkte anderer Höhenlagen. Zudem lässt sich feststellen, dass die Schälquoten der Höhenklassen ab einer Höhe von 300 Metern bis hin zu Lagen von knapp unter 700 Metern generell recht hoch sind und stets bei Werten von über 4,8 \% liegen (im Mittel bei 5,34 \%). In den tieferen Lagen von unter 300 Metern sowie ab einer Höhe von 700 Metern reichen die Klassenwerte hingegen nur noch von 0,74 bis 3,21\%. Das mittlere Schälprozent dieser Höhenbereiche bleibt mit einem Wert von 1,96 \% somit deutlich und hochsignifikant $(p<0,001)$ hinter dem Schälprozent der mittleren Höhenlagen zurück. Abbildung 4.4 veranschaulicht die zur Höhenlage im Harz festgestellten Unterschiede der Schälintensität.

Analog zum Harz wurde auch im Solling eine Stratifizierung nach der Höhe der Stichprobenpunkte in Intervallen von 100 Metern vorgenommen. Hieraus ließen sich vier Höhenklassen ableiten (siehe ebenfalls Tab. 4.5). Die Klassengrenzen wurden dem Aufkommen der dort erfassten Stichprobenpunkte angepasst und unterscheiden sich somit von denen des Harzes. Auch im Solling zeigen sich die geringsten Schälprozentwerte in der Klasse der tiefsten dokumentierten Lagen (145 bis $239 \mathrm{~m}$ ), doch sind die Mittelwertabweichungen der Klassen voneinander in diesem Fall nicht signifikant. Gleiches gilt für die Abgrenzung der mit 3,74 \% Schälquote vermeintlich am stärksten geschädigten Höhenlage zwischen 240 und 339 Metern.

\subsubsection{Exponiertheit der Standorte}

Zur Überprüfung der Annahme, dass an Standorten geringer Exponiertheit höhere Schälprozente registriert werden, wurde der Datenpool zunächst in vier Klassen unter- 
Tabelle 4.6: Mittleres Neuschälprozent an Fichte im Harz, bezogen auf Klassen der Exponiertheit der weiteren Umgebung; berechnet über sämtliche Stichprobenpunkte mit Fichtenvorkommen und alle drei Inventurjahre; getrennt dargestellt für drei Varianten der Klassengrenzen; $n=1.705$

\begin{tabular}{lrrrrr}
\hline Exponiertheit & $n$ & $\bar{x}$ & $s$ & $V$ & $V_{r}$ \\
\hline 0-Wert & 121 & $8,02 \%$ & 19,40 & 2,42 & $22,01 \%$ \\
1 bis 9 & 626 & $5,56 \%$ & 13,16 & 2,37 & $9,46 \%$ \\
10 bis 23 & 882 & $3,67 \%$ & 9,23 & 2,51 & $8,47 \%$ \\
24 bis 36 & 76 & $7,13 \%$ & 14,29 & 2,01 & $23,00 \%$ \\
0-Wert & 121 & $8,02 \%$ & 19,40 & 2,42 & $22,01 \%$ \\
1 bis 36 & 1.584 & $4,59 \%$ & 11,36 & 2,45 & $6,16 \%$ \\
0 bis 10 & 812 & $5,78 \%$ & 14,02 & 2,43 & $8,52 \%$ \\
11 bis 36 & 893 & $3,97 \%$ & 9,84 & 2,48 & $8,30 \%$ \\
\hline
\end{tabular}

$n=$ Anzahl der Stichprobenpunkte, $\bar{x}=$ arithmetisches Mittel der Neuschäle, $s=$ Standardabweichung, $V=$ Variationskoeffizient, $V_{r}=$ relativer Variationskoeffizient

teilt. Im oberen Segment der Tabelle 4.6 sind die Klassenwerte für den Harz aufgeführt. Da immerhin 121 Stichprobenpunkte den Exponiertheitswert 0 tragen, wurden diese Punkte in einer eigenen Klasse belassen. Um den Bereich der gering exponierten Standorte weiter spezifizieren zu können, wurde die nachfolgende Klasse mit Werten zwischen 1 und 9 etwas enger gefasst und der Bereich der höheren Parameterwerte schließlich in zwei weitere Klassen aufgeteilt. Die mittleren Schälprozente der vier Klassen variieren von 3,7 \% bis 8,0 \%. In der Varianzanalyse werden die Mittelwertabweichungen untereinander als insgesamt hochsignifikant ausgewiesen, wobei der Mittelwertvergleich nach Tukey hierin signifikante Unterschiede zwischen den Klassen 0 und 1-9 sowie zwischen den Klassen 1-9 und 10-23 annimmt.

Bei der Zweiteilung des Datenpools (mittleres Segment Tab. 4.6) liegt das gemittelte Schälprozent der Klasse 0 mit 8,0 \% etwa 1,7-fach über dem der restlichen Klassen mit einem Wert von 4,6 \%. Dieser ebenfalls hochsignifikante Unterschied beinhaltet jedoch einen hohen relativen Variationskoeffizienten in der Klasse 0. Erweitert man diese Klasse um Werte bis 10 (unteres Tabellensegment), bleibt der signifikante Unterschied in der Schälquote bei nun geringerer Varianz erhalten. Standorte schwächerer Exponiertheit bis einschließlich des Wertes 10 weisen demnach ein 1,5-fach höheres Neuschälprozent auf, als solche mit entsprechend höherer Exponiertheit. Das hohe Schälprozent von 
Tabelle 4.7: Mittleres Neuschälprozent an Fichte im Solling, bezogen auf Klassen der Exponiertheit der weiteren Umgebung; berechnet über sämtliche Stichprobenpunkte mit Fichtenvorkommen und alle drei Inventurjahre; getrennt dargestellt für drei Varianten der Klassengrenzen; $n=2.258$

\begin{tabular}{lrrrrr}
\hline Exponiertheit & $n$ & $\bar{x}$ & $s$ & $V$ & $V_{r}$ \\
\hline 0 bis 9 & 353 & $2,41 \%$ & 8,25 & 3,42 & $18,23 \%$ \\
10 bis 23 & 1126 & $3,29 \%$ & 10,68 & 3,25 & $9,68 \%$ \\
24 bis 35 & 522 & $3,20 \%$ & 9,28 & 2,90 & $12,71 \%$ \\
36 & 257 & $2,61 \%$ & 6,92 & 2,65 & $16,51 \%$ \\
\hline
\end{tabular}

$n=$ Anzahl der Stichprobenpunkte, $\bar{x}=$ arithmetisches Mittel der Neuschäle, $s=$ Standardabweichung, $V=$ Variationskoeffizient, $V_{r}=$ relativer Variationskoeffizient

$7,1 \%$ der Klasse 24 bis 36 setzt sich hingegen nicht signifikant $(p=0,15)$ vom Rest der Stichprobenpunkte ab.

Bei der Einteilung der Sollingdaten in Klassen unterschiedlicher Exponiertheit wurde in gleicher Weise wie im Harz verfahren. Da hier jedoch lediglich 16 Stichprobenpunkte den Wert 0 tragen, wurde auf eine seperate Klasse hierfür verzichtet und die Grenze entsprechend auf die nächste Stufe erweitert (0 bis 9). Weil sich aber allein 257 Punkte mit dem Wert 36 im Datenset befinden, konnte stattdessen eine gegenpolige Extremklasse gebildet werden. In Tabelle 4.7 sind die vier ausgewiesenen Klassen mit ihren jeweiligen Werten aufgeführt. Die mittleren Schälprozente der Klassen liegen mit Werten zwischen 2,4 \% und 3,3\% enger beieinander als die Werte im Harz. Zudem sind die Varianzen recht hoch, sodass die Unterschiede insgesamt nach durchgeführter Varianzanalyse als nicht signifikant angesehen werden müssen $(p=0,41)$.

\subsubsection{Hangneigung des Geländes}

Anhand der unter 3.1.2 beschriebenen Einteilung der Hänge in die Klassen „schwach“, „mäßig“ und „stark“ geneigt sind die jeweiligen mittleren Neuschälprozente über alle drei Inventurjahre gemeinsam ermittelt worden. In Tabelle 4.8 sind die Mittelwerte mit zugehörigen relativen Variationskoeffizienten der Klassen sowie die Signifikanzwerte der jeweiligen Varianzanalyse je Datenset aufgeführt. Auf Basis der zugrunde liegenden Klassengrenzen zeigt sich ein uneinheitliches Bild. Bei der Gesamtberechnung der Sollingdaten (unteres Segment der Tabelle) stellen sich keine als signifikant geltenden 
Unterschiede der Mittelwerte zwischen den Klassen ein $(p=0,064)$. Gleiches gilt für das homogene Datenset aus dem Solling. Der heterogene Datenpool dieses Gebietes zeigt hingegen ein statistisch gesichertes $(p=0,017)$ höheres Neuschälprozent in den schwach geneigten Lagen. In dieser Klasse bleibt die realtive Varianz der einzelnen Mittelwerte zudem am geringsten. So ergibt sich für Hänge mit weniger als $9 \%$ Steigung ein Schälprozentwert von 3,7\% gegenüber nur 2,2 \% Neuschäle in Bereichen ab $9 \%$ Steigung. Eine optimale Anpassung der Klassengrenzen an die Verteilung der Stichprobenpunkte bietet diese Gliederung jedoch möglicherweise noch nicht, zumal die Klasse der mäßig geneigten Standorte sehr groß ist (vgl. Abb. 3.3.).

Für den Harz ergeben sich andere Schwerpunkte. Sowohl innerhalb der Gesamtstichprobe als auch innerhalb des homogenen Datensets zeigen sich signifikante Mittelwertunterschiede zwischen den Klassen, nicht jedoch für das heterogene Datenset. Anders als im Solling liegen die signifikanten Unterschiede im Harz jedoch zwischen der höchsten Hangneigungsklasse und den sich darunter befindenden Werten. Zudem werden an Standorten mit starker Hangneigung, also mit über $17 \%$ Steigung, im Mittel deutlich höhere Neuschälprozente festgestellt als an den schwach bis mäßig geneigten Standorten (Zusammenfassung der beiden unteren Klassen). Es liegt somit ein gegenteiliger Trend zum Solling vor. Vergleicht man anhand der Gesamtstichprobe aus homogenen und heterogenen Datensets das Neuschälaufkommen schwach und mäßig geneigter Standorte mit dem stärker geneigter Orte, beträgt die Mittelwertabweichung

Tabelle 4.8: Mittleres Winterneuschälprozent an Fichte in Harz und Solling 2002-2006, bezogen auf Hangneigungsklassen

\begin{tabular}{|c|c|c|c|c|c|c|c|}
\hline \multirow{2}{*}{$\begin{array}{l}\text { Hangneigung } \\
\text { Datenset }\end{array}$} & \multirow[b]{2}{*}{ Sig. } & \multicolumn{2}{|c|}{ schwach } & \multicolumn{2}{|c|}{$m \ddot{a} ß i g$} & \multicolumn{2}{|c|}{ stark } \\
\hline & & $\bar{x}$ & $V_{r}$ & $\bar{x}$ & $V_{r}$ & $\bar{x}$ & $V_{r}$ \\
\hline Solling homogen & 0,186 & $2,86 \%$ & $(8,42 \%)$ & $3,60 \%$ & $(15,14 \%)$ & $2,08 \%$ & $(33,95 \%)$ \\
\hline Solling heterogen & 0,046 & $3,71 \%$ & $(11,55 \%)$ & $2,45 \%$ & $(20,83 \%)$ & $1,80 \%$ & $(36,85 \%)$ \\
\hline Harz homogen & 0,001 & $3,84 \%$ & $(16,41 \%)$ & $2,59 \%$ & $(14,70 \%)$ & $5,29 \%$ & $(8,90 \%)$ \\
\hline Harz heterogen & 0,271 & $4,56 \%$ & $(22,63 \%)$ & $4,10 \%$ & $(16,50 \%)$ & $5,88 \%$ & $(11,17 \%)$ \\
\hline Solling gesamt & 0,064 & $3,34 \%$ & $(7,90 \%)$ & $2,85 \%$ & $(13,44 \%)$ & $1,87 \%$ & $(27,71 \%)$ \\
\hline Harz gesamt & 0,002 & $4,21 \%$ & $(14,50 \%)$ & $3,28 \%$ & $(11,37 \%)$ & $5,64 \%$ & $(7,70 \%)$ \\
\hline
\end{tabular}

Sig. $=p$-Wert, $\bar{x}=$ arithmetisches Mittel der Neuschäle, $V_{r}=$ relativer Variationskoeffizient; schwach $<9 \%$, mittel $=9-17 \%$, stark $>17 \%$ 
Tabelle 4.9: Mittleres Winterneuschälprozent an Fichte in Harz und Solling 2002-2006, bezogen auf Hangneigungsklassen; Median als Klassengrenze

\begin{tabular}{llrrrrrr}
\hline Gebiet & Sig. & Hangklasse & $n$ & $\bar{x}$ & $s$ & $V$ & $V_{r}$ \\
\hline \multirow{2}{*}{ Solling } & 0,004 & $<7 \%$ & 1.145 & $3,62 \%$ & 10,54 & 2,91 & $8,59 \%$ \\
& & $\geq 7 \%$ & 1.113 & $2,42 \%$ & 8,58 & 3,48 & $10,44 \%$ \\
\multirow{3}{*}{ Harz } & 0,001 & $<20 \%$ & 863 & $3,72 \%$ & 8,95 & 2,41 & $8,19 \%$ \\
& & $\geq 20 \%$ & 842 & $5,97 \%$ & 14,46 & 2,42 & $8,35 \%$ \\
\hline
\end{tabular}

Sig. $=p$-Wert, $n=$ Anzahl der Stichprobenpunkte, $\bar{x}=$ arithmetisches Mittel der Neuschäle, $s=$ Standardabweichung, $V=$ Variationskoeffizient, $V_{r}=$ relativer Variationskoeffizient

rund $50 \%$ (von 3,7\% auf 5,6\% bei starker Steigung; $p=0,001$ ). Bei der homogenen Stichprobe sind es sogar knapp $70 \%$ (von 3,1\% auf 5,3\%; $p<0,001$ ).

Legt man für die Klasseneinteilung in beiden Gebieten jeweils den Median der Hangneigung zugrunde und bildet an dieser Grenze zwei Gruppen, ergeben sich klare, robustere Ergebnisse. Im Solling liegt der Median bei einem Steigungswert von 6,9 \%, was also als neue Klassengrenze gewählt wird. Mit einem mittleren Schälprozent von 3,6 \% liegt der Wert der weniger geneigten Hänge und Plateaulagen nun auch für die Gesamtstichprobe signifikant über dem der steileren Hänge mit lediglich 2,4 \% (Tab. 4.9). Die Varianzen innerhalb der Klassen bleiben dabei auf niedrigerem Niveau als zuvor. An Standorten mit höherer Hangneigung verringert sich das Neuschälaufkommen somit um rund ein Drittel. In Abbildung 4.5 auf Seite 67 sind die Mittelwerte beider Klassen mit ihren jeweils zugehörigen Informationen veranschaulichend dargestellt.

Für den Harz wird die Klassengrenze entsprechend der dort vorherrschenden höheren Hangneigungswerte angepasst. Ausgehend vom Median (19,9\%) wird der Datenpool der Gesamtstichprobe auf je eine Klasse unter 20 \% Hangneigung und eine ab $20 \%$ Hangneigung aufgeteilt (ebenfalls Tab. 4.9). Auch hier ergeben sich nun gleichmäßig niedrige Varianzen. Das mittlere Neuschälprozent der steileren Lagen erreicht annähernd einen Wert von 6 \%. Gegenüber dem Schälprozent der schwächer geneigten Standorte $(3,7 \%)$ entspricht dies einer Zunahme um $60 \%$. Eine bildhafte Darstellung findet sich ebenfalls in Abbildung 4.5 . 


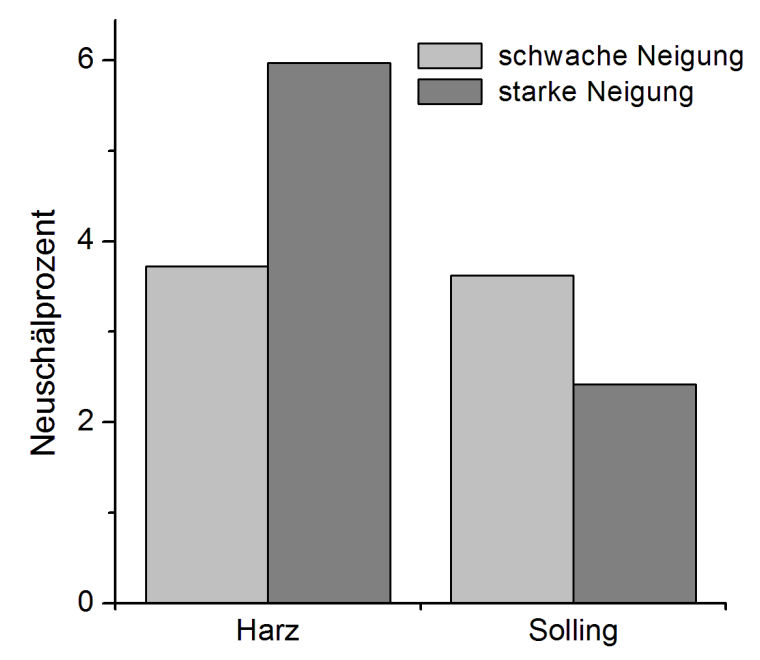

Abbildung 4.5: Mittlere Neuschälprozente nach Hangneigung getrennt für beide Untersuchungsgebiete mit dem Median als Klassengrenze; schwache Neigung Harz $<20 \%$, Solling $<7 \%$

\subsubsection{LUV- und LEE-Lage}

Zum Vergleich der einzelnen Hangrichtungsklassen wurden diese in zwei Gruppen eingeteilt. Mit den Klassen Südwesten, Westen und Nordwesten wird die Gruppe der LUV-Lage umschrieben, da Winde aus diesen Richtungen in beiden Gebieten vorherrschen. Dieser Gruppe wird die Summe der restlichen Klassen gegenübergestellt. Diese Einteilung trennt den Datenpool in jeweils vergleichbar große Teile. Einen Vergleich der mittleren Neuschälprozente bietet hierzu Tabelle 4.10. Im oberen Bereich der Tabelle sind die Werte für den Solling notiert. Für die Wetterseite, also die erste Gruppe, ergibt sich ein Winterneuschälprozent von 2,97 \%. Für die wetterabgewandte Referenzklasse wird ein leicht höheres Schälprozent von 3,13 \% ermittelt. Der Unterschied dieser beiden Mittelwerte liegt jedoch weit außerhalb des Signifikanzbereiches.

Im Harz setzt sich das mittlere Neuschälprozent der LUV-Gruppe mit 5,44 \% deutlicher von der LEE-Gruppe mit 4,04 \% ab und der festgestellte Unterschied kann als statistisch signifikant gelten $(p=0,018)$. Die dem Wetter mutmaßlich verstärkt ausgesetzten Standorte weisen im Harz somit eine etwas geringere Schälbelastung auf. 
Tabelle 4.10: Mittleres Winterneuschälprozent an Fichte in Harz und Solling 2002-2006, bezogen auf Expositionsklassen

\begin{tabular}{lllrrrrr}
\hline Gebiet & Sig. & Exposition & $n$ & $\bar{x}$ & $s$ & $V$ & $V_{r}$ \\
\hline \multirow{2}{*}{ Solling } & \multirow{2}{*}{0,703} & SW-W-NW & 1.027 & $2,97 \%$ & 9,01 & 3,03 & $9,47 \%$ \\
& & N bis S & 1.228 & $3,13 \%$ & 10,15 & 3,25 & $9,27 \%$ \\
\multirow{2}{*}{ Harz } & \multirow{2}{*}{0,018} & SW-W-NW & 729 & $4,04 \%$ & 10,22 & 2,53 & $9,38 \%$ \\
& & N bis S & 973 & $5,44 \%$ & 13,23 & 2,43 & $7,80 \%$ \\
\hline
\end{tabular}

Sig. $=p$-Wert, $n=$ Anzahl der Stichprobenpunkte, $\bar{x}=$ arithmetisches Mittel der Neuschäle, $s=$ Standardabweichung, $V=$ Variationskoeffizient, $V_{r}=$ relativer Variationskoeffizient

\subsubsection{Strahlungsinput am Standort}

Die potentielle Sonnenstrahlung am Stichprobenpunkt ist in 10 Klassen skaliert. In der Annahme, dass Orte mit hohem Strahlungsinput präferiert sein könnten, werden diese Klassen in zwei Gruppen zusammengefasst. Dabei wird die Klassen mit Werten von 0 bis 6 Stunden Strahlungsinput denen mit besonders hohem Strahlungsgenuss gegenübergestellt. Im Solling ergibt sich für diese Gruppe ein mittleres Neuschälprozent von 3,17 \%, während im Datenpool der geringeren Strahlungsklassen nur ein Schälprozent von 1,75\% erreicht wird (Tab.4.11). Der Unterschied ist statistisch gesehen jedoch nicht signifikant $(p=0,060)$. Im Harz zeigt sich ein gegenteiliges Ergebnis. Stichprobenpunkte an Standorten mit potentiell höherem Strahlungsgenuss (7 bis 9 Stunden) weisen dort ein etwas geringeres Neuschälprozent auf, als solche an strahlungsärmeren Standorten (5,88 \% zu 4,15 \%). Kehrt man das Verhältnis der Gruppeneinteilung um und setzt die Klasse 0 gegen alle anderen Klassen (Tab. 4.11 unten), verstärkt sich dieser Effekt noch. Die Schälquote in der strahlungsärmsten Klasse liegt mit 8,27 \% deutlich über der der restlichen Klassen (4,64 \%). Die Mittelwertabweichungen beider Vergleiche können dabei als statistisch signifikant gelten.

\subsection{Jagdstreckendaten als Indikatoren für Schälschäden}

\subsubsection{Korrelation der Schälrate mit Höhe der Jagdstrecke}

Unterzieht man die einzelnen Reviere mit ihrem jeweiligen Rotwildindex einer Varianzanalyse, ergeben sich insgesamt signifikante Unterschiede innerhalb beider Untersuchungsgebiete. Gleiches gilt in Bezug auf die revierbezogenen Neuschälraten. Dennoch 
Tabelle 4.11: Mittleres Winterneuschälprozent an Fichte in Harz und Solling 2002-2006, bezogen auf Strahlungsklassen

\begin{tabular}{llcrrrrr}
\hline Gebiet & Sig. & Stunden & $n$ & $\bar{x}$ & $s$ & $V$ & $V_{r}$ \\
\hline \multirow{2}{*}{ Solling } & 0,060 & $0-6$ & 180 & $1,75 \%$ & 7,76 & 4,42 & $32,96 \%$ \\
& & $7-9$ & 2.078 & $3,17 \%$ & 9,78 & 3,09 & $6,78 \%$ \\
\multirow{3}{*}{ Harz } & 0,004 & $0-6$ & 667 & $5,88 \%$ & 14,78 & 2,51 & $9,73 \%$ \\
& & $7-9$ & 1.038 & $4,15 \%$ & 9,84 & 2,37 & $7,35 \%$ \\
& 0,005 & 0 & 90 & $8,27 \%$ & 22,57 & 2,73 & $28,77 \%$ \\
& & $1-9$ & 1.615 & $4,64 \%$ & 11,15 & 2,40 & $5,98 \%$ \\
\hline
\end{tabular}

Sig. $=p$-Wert, $n=$ Anzahl der Stichprobenpunkte, $\bar{x}=$ arithmetisches Mittel der Neuschäle, $s=$ Standardabweichung, $V=$ Variationskoeffizient, $V_{r}=$ relativer Variationskoeffizient

Tabelle 4.12: Mittlerer Jagdstreckenindex der Hauptbejagungszeit und mittleres Winterneuschälprozent an Fichte je Forstamt in Harz und Solling 2002-2006

\begin{tabular}{rccrrrrrr}
\hline Gebiet & Sig. & Forstamt & $n$ & $\bar{x}$ Jagd & $\bar{x}$ Schäle & $s$ & $V$ & $V_{r}$ \\
\hline \multirow{2}{*}{ Harz } & \multirow{2}{*}{0,001} & I & 330 & 0,88 & $5,32 \%$ & 11,57 & 2,17 & $11,96 \%$ \\
& & II & 358 & 1,05 & $7,88 \%$ & 17,74 & 2,25 & $11,89 \%$ \\
& III & 342 & 1,03 & $4,60 \%$ & 10,89 & 2,37 & $12,79 \%$ \\
& IV & 269 & 0,65 & $2,36 \%$ & 9,02 & 3,83 & $23,33 \%$ \\
& IV & 406 & 0,76 & $2,56 \%$ & 7,49 & 2,10 & $10,44 \%$ \\
\multirow{2}{*}{ Solling } & & & & & & & \\
& & I 001 & 1.001 & 0,70 & $3,56 \%$ & 10,63 & 2,99 & $9,45 \%$ \\
& II & 595 & 0,49 & $1,99 \%$ & 7,82 & 3,93 & $16,11 \%$ \\
& III & 662 & 0,47 & $3,24 \%$ & 9,48 & 2,92 & $11,36 \%$ \\
\hline
\end{tabular}

Sig. $=p$-Wert, $n=$ Anzahl der Stichprobenpunkte, $\bar{x}$ Jagd = arithmetisches Mittel vom Jagdstreckenindex in Stk./100 ha, $\bar{x}$ Schäle = arithmetisches Mittel der Neuschäle, $s=$ Standardabweichung, $V=$ Variationskoeffizient, $V_{r}=$ relativer Variationskoeffizient; $n, s, V$ und $V_{r}$ beziehen sich auf $\bar{x}$ Schäle

sind eine Berechnung und ein Vergleich auf dieser Ebene nicht sinnvoll, da die vermeintlich robusten Werte lediglich durch große Unterschiede in den Mittelwerten der Schälschäden begründet sind, zugleich aber eine viel zu geringe Stichprobengröße (im Harz durchschnittlich unter 35 Punkte je Revier) vorliegt. Die mittleren Schälprozente der Reviere können somit nicht als repräsentativ gelten und stehen für die vergleichende Statistik mit den Revierstreckendaten leider nicht zur Verfügung. Die folgenden Betrachtungen müssen deshalb auf Ebene der Forstamtsgrenzen erfolgen. 
Tabelle 4.13: Gegenüberstellung des jährliches mittleren Rotwildstreckenaufkommens im Zeitraum September bis Januar mit dem jeweiligen Neuschälprozent der Periode

\begin{tabular}{lrrrrrrr}
\hline Gebiet & Periode & $n$ Jagd & $n$ Schäle & $\bar{x}$ Jagd & $\bar{x}$ Schäle & $V_{r}$ Jagd & $V_{r}$ Schäle \\
\hline \multirow{2}{*}{ Harz } & $2001 / 02$ & 49 & 456 & 1,11 & $4,57 \%$ & $11,36 \%$ & $11,47 \%$ \\
& $2003 / 04$ & 49 & 611 & 1,31 & $4,91 \%$ & $9,66 \%$ & $10,11 \%$ \\
& $2005 / 06$ & 49 & 638 & 1,29 & $4,94 \%$ & $8,77 \%$ & $9,95 \%$ \\
Solling & $2001 / 02$ & 27 & 749 & 0,72 & $3,99 \%$ & $16,82 \%$ & $9,46 \%$ \\
& $2002 / 03$ & 27 & 758 & 0,71 & $3,11 \%$ & $15,71 \%$ & $12,25 \%$ \\
& $2005 / 06$ & 27 & 751 & 0,38 & $2,06 \%$ & $18,93 \%$ & $13,75 \%$ \\
\hline
\end{tabular}

$n=$ Anzahl, $\bar{x}$ Jagd = arithmetisches Mittel der Rotwilderlegungen in Stück je 100 ha, $\bar{x}$ Schäle = arithmetisches Mittel der Neuschäle, $V_{r}=$ relativer Variationskoeffizient

Auch von den Revieren auf das zugehörige Forstamt gemittelt weist der Jagdstreckenindex noch eine Differenzierung auf, wenngleich diese erwartungsgemäß geringer ausfällt als zwischen den einzelnen Revieren. So zeigen sich sowohl innerhalb des Harzes als auch im Solling signifikante Unterschiede zwischen den Forstämtern (siehe Tab. 4.12). Die Spanne des im Forstamtsbereich erlegten Rotwildes reicht im Harz von 0,7 bis 1,1 und im Solling von 0,5 bis 0,7 Stücken je 100 Hektar. Die Werte der mittleren Neuschäle liegen im Harz zwischen 2,4 \% und 7,9 \%. Die zugehörigen Werte des relativen Variationskoeffizienten befinden sich auf untereinander vergleichbarem Niveau. Lediglich jener des niedrigsten Mittelwertes weist, wohl aufgrund des dort geringeren Stichprobenumfangs, auf die generell vorhandene Varianz der Daten hin. Im Solling bleiben die mittleren Schälprozente bekanntermaßen moderat. Bezogen auf die Forstämter liegen sie zwischen $2 \%$ und 3,6 \% . Die $V_{r}$-Werte zeigen sich uneinheitlicher als im Harz, was wiederum in den unterschiedlichen Stichprobengrößen der drei Forstämter begründet sein dürfte. Bereits beim Vergleich der Mittelwertpaare in Tabelle 4.12 werden Parallelen deutlich. Hohen Streckenwerten stehen auch hohe mittlere Neuschälprozente gegenüber. So zeigen sich auch bei Korrelationsrechnungen statistische Zusammenhänge dieser beiden Paramater. Für die fünf Wertepaare im Harz ergibt sich ein Korrelationskoeffizient von 0,836 , was einen recht starken Zusammenhang vermuten lässt. Mit einem $r^{2}$-Wert von 0,573 bleibt die Aussagekraft der Sollingdaten diesbezüglich zurück.

Klassifiziert man die beiden Parameter nicht anhand von Forstamtsgrenzen, sondern zwischen den Inventurjahren, zeichnen sich ebenfalls Parallelen in deren Ausprägung 
4.3. Jagdstreckendaten als Indikatoren für Schälschäden

Tabelle 4.14: Mittleres Winterneuschälprozent an Fichte in Harz 2002-2006, bezogen auf Nähe zu Wanderwegen

\begin{tabular}{lccrrrrr}
\hline Gebiet & Sig. & Klasse & $n$ & $\bar{x}$ & $s$ & $V$ & $V_{r}$ \\
\hline \multirow{2}{*}{ Harz } & 0,233 & 0 & 987 & $5,28 \%$ & 12,67 & 2,40 & $7,64 \%$ \\
& & 1 & 250 & $4,85 \%$ & 14,08 & 2,90 & $18,35 \%$ \\
& 2 & 315 & $3,91 \%$ & 9,84 & 2,51 & $14,16 \%$ \\
& \multirow{2}{*}{0,146} & 0 & 987 & $5,28 \%$ & 12,67 & 2,40 & $7,64 \%$ \\
& $1+2$ & 565 & $4,33 \%$ & 11,90 & 2,75 & $11,56 \%$ \\
& 0,102 & $0+1$ & 1.237 & $5,19 \%$ & 12,96 & 2,50 & $7,10 \%$ \\
& 2 & 315 & $3,91 \%$ & 9,84 & 2,51 & $14,16 \%$ \\
\hline
\end{tabular}

Sig. $=p-$ Wert,$n=$ Anzahl der Stichprobenpunkte, $\bar{x}=$ arithmetisches Mittel der Neuschäle, $s=$ Standardabweichung, $V=$ Variationskoeffizient, $V_{r}=$ relativer Variationskoeffizient

ab. In Tabelle 4.13 sind die zuvor bereits einzeln dokumentierten Jahresmittelwerte der Neuschäle nun denen der jeweiligen Streckendaten der Jahrgänge gegenübergestellt. Die Werte der Neuschälprozente folgen dabei sehr eng den für die entsprechende Periode ermittelten Erlegungszahlen je 100 Hektar. Lokal unterschieden wird hierbei nur noch nach Gesamtgebiet. Mit einem Koeffizienten von $r^{2}=0,987$ korrelieren die Werte im Harz erneut am stärksten, doch auch im Solling zeigt sich eine statistisch sehr eng verlaufende Beziehung zwischen Streckenergebnis und Schälschädigungsrate. Dort liegt der entsprechende Wert bei $r^{2}=0,902$. In beiden Untersuchungsgebieten ergeben sich somit höhere $r^{2}$-Werte bei der Betrachtung der Gesamtentwicklung eines Gebietes, also über Forstamtsgrenzen hinweg, als bei der Unterscheidung der einzelnen Forstämter.

\subsubsection{Nähe zu Wanderwegen als Kriterium}

Die Werte in Tabelle 4.14 geben Auskunft über die Ergebnisse zur Mittelwertberechnung der Neuschälprozente innerhalb der drei Klassen. Es zeigt sich zwar zunächst eine Abnahme des mittleren Neuschälaufkommens von Standorten nahe schwach frequentierten hin zu stark frequentierten Wanderwegen, doch kann diese geradlinige Tendenz nach Durchführung einer Varianzanalyse nicht als signifikant gewertet werden $(p>0,05)$. Statistisch etwas belastbarer, aber dennoch nicht signifikante Unterschiede zeigen sich beim Vergleich der Klasse 0 mit den übrigen Daten und beim Vergleich der Klasse 2 mit der Summe aus den Klassen 0 und 1. In beiden Varianten wird 
die aufgezeigte Tendenz bestärkt, dass abseits intensiv genutzter Wanderwege höhere Neuschälprozente auftreten, statistisch abgesichert ist diese Annahme jedoch nicht. 


\section{Vorbetrachtung der Chancenverhältnisse}

In Vorbereitung auf die Interpretation der nachfolgenden logistischen Regressionsmodelle sollen zunächst die Chancenverhältnisse der jeweiligen Faktoren einzeln dargestellt werden. Die Herleitung der Chancenverhältnisse selbst wird in Kapitel 2.4.3 auf Seite 31 ausgiebig beschrieben. Bei den folgenden Darstellungen handelt es sich also um die so genannten odds ratios, welche analog zu den Berechnungen der Regressionsmodelle hergeleitet werden. Somit beziehen sich die grafischen Abweichungen vom Mittelwert explizit nicht auf die Differenz der Schälprozente einzelner Klassen, sondern auf deren abweichendes Chancenverhältnis (odds) vom mittleren Chancenverhältnis über alle Klassen hinweg. Das odds ratio (also „odds der Klasse“ / „odds gesamt“) nimmt dabei Werte um 1 an. Bei einem odds ratio von 1,35 wird die Abweichung in Form von $+35 \%$ dargestellt, bei einem Wert von 0,75 hingegen als Abweichung von -25\%. Es handelt also lediglich um eine Umrechnung in Prozentwerte zur besseren Darstellung der odds ratios.

\subsection{Ergebnisse}

\subsubsection{Altschäle}

Betrachtet man die Abweichungen der Chancenverhältnisse einzelner Parameterklassen der Altschäle in Bezug zum mittleren Chancenverhältnis der Gesamtstichprobe, zeigt sich im Harz (Abb. 5.1) eine klare Tendenz. Bei nur wenigen zuvor durch Schälschäden belasteten Bäumen liegt die Neuschälwahrscheinlichkeit dort unterhalb des Mittelwertes. Erst ab etwa 12 vorgeschälten Bäumen besteht dann ein deutlich höheres Risiko für Neuschäle im Stichprobenpunkt. Weisen sämtliche Bäume vor Ort Altschälschäden auf, verschiebt sich das lokale Chancenverhältnis im Vergleich zum Gesamtdurchschnitt um etwa 100 \%. Im Solling liegt eine vergleichbare Tendenz wie im Harz vor (Abb. 5.2). Es ergibt sich aber ein insgesamt weniger ausgeprägtes Bild. Auffällig ist hier vor allem das stark reduzierte Neuschälrisiko, wenn gar keine Vorschäle am Punkt vorhanden ist. 


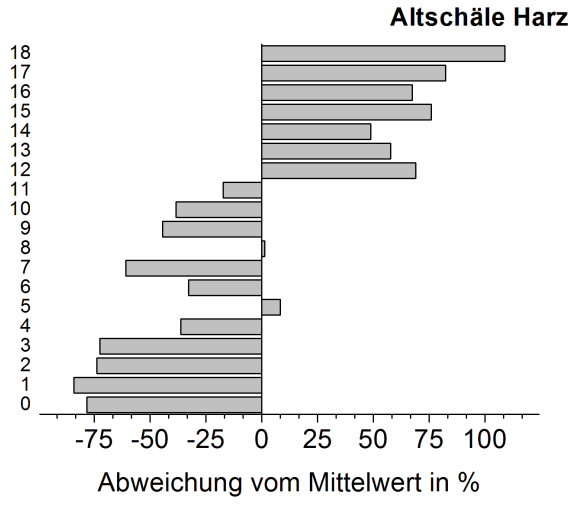

Abbildung 5.1: Relative Abweichung der mittleren Schälprozente einzelner Klassen des Altschälschadenaufkommens vom Gesamtschälprozent im Harz; $\bar{x}=4,45 \%$

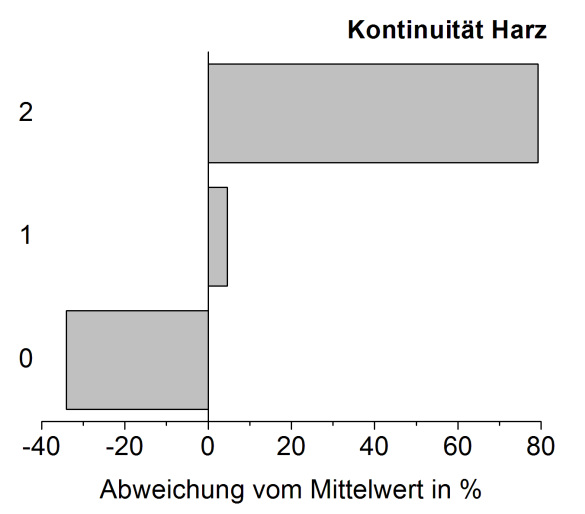

Abbildung 5.3: Relative Abweichung der mittleren Schälprozente einzelner Kontinuitätsklassen vom Gesamtschälprozent im Harz; $\bar{x}=5,01 \%, n=195$

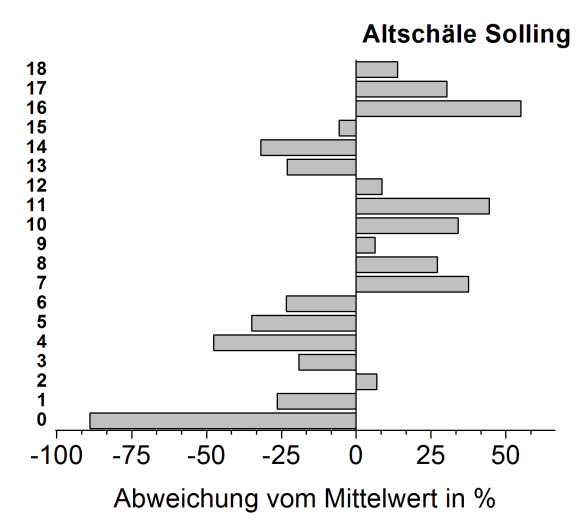

Abbildung 5.2: Relative Abweichung der mittleren Schälprozente einzelner Klassen des Altschälschadenaufkommens vom Gesamtschälprozent im Solling; $\bar{x}=2,95 \%$

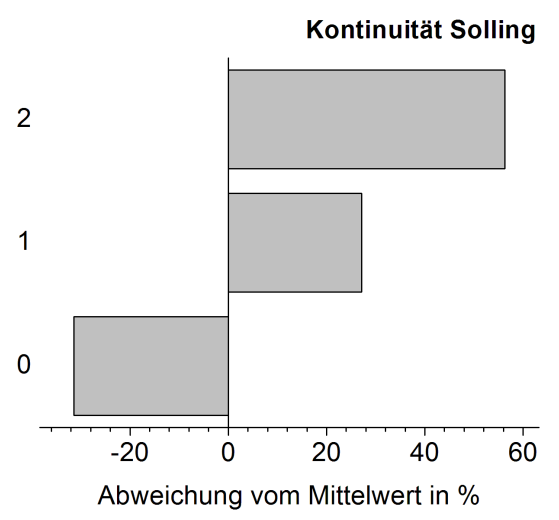

Abbildung 5.4: Relative Abweichung der mittleren Schälprozente einzelner Kontinuitätsklassen vom Gesamtschälprozent im Solling; $\bar{x}=2,07 \%, n=258$

\subsubsection{Kontinuität der Schädigung}

In beiden Untersuchungsgebieten verschiebt sich das Chancenverhältnis zu mehr Neuschäle an Orten mit höherer Kontinuität (Abb. 5.3 und 5.4). Nach der bereits durchgeführten Analyse vorangegangener Schäle über den Faktor Altschäle bestätigt sich hier also deren Tendenz. Im Vergleich der beiden Gebiete zeigen sich keine nennenswerten Untersschiede. Im Solling weicht die zweite Parameterklasse lediglich deutlicher vom Mittelwert ab als im Harz, wo wiederum der Sprung von der zweiten zur dritten Klasse stärker ausgeprägt ist. 


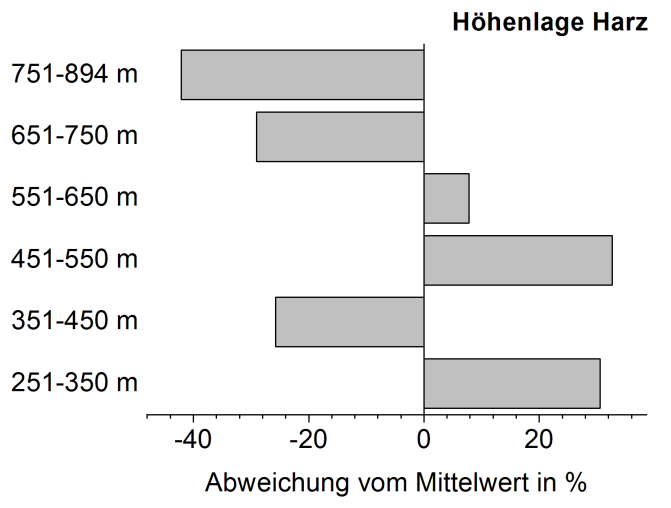

Abbildung 5.5: Relative Abweichung der mittleren Schälprozente einzelner Klassen der Höhenlage ü.NN vom Gesamtschälprozent im Harz; $\bar{x}=4,25 \%$, $n=773$

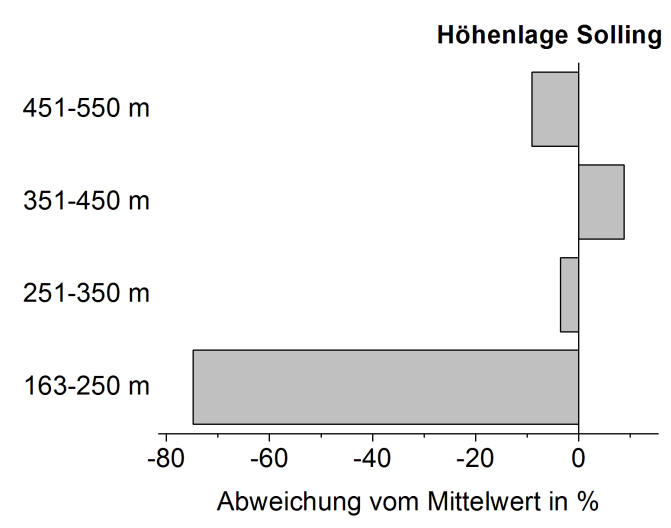

Abbildung 5.6: Relative Abweichung der mittleren Schälprozente einzelner Klassen der Höhenlage ü.NN vom Gesamtschälprozent im Solling; $\bar{x}=2,95 \%$, $n=891$

\subsubsection{Höhenlage}

Durch die Klassifizierung nach Höhenstufen ergibt sich im Harz kein klares Bild bei der Betrachtung der Chancenverhältnisse. In den höchsten Lagen ab etwa $650 \mathrm{Me}-$ tern zeigt sich zwar ein tendenziell geringeres Neuschälrisiko als im Mittel (Abb. 5.5 und in niedrigeren Lagen liegt die Schälwahrscheinlichkeit eher über dem mittleren Verhältnis, doch lässt sich insgesamt kein enger Zusammenhang ablesen. Im Solling liegen alle Chancenverhältnisse um den Mittelwert (Abb. 5.6. Lediglich in der Klasse der tiefsten Lagen weicht das Schälrisiko deutlich vom mittleren Verhältnis ab. Das Neuschälvorkommen nimmt dort einen sehr viel geringeren Anteil der Stichprobe ein. Jedoch ist der Umfang dieser Klasse mit 270 Datensetzen (von 16.128 insgesamt) auch am geringsten.

\subsubsection{Hangneigung}

Bei der vorliegenden Einteilung in Hangneigungsklassen zeigt sich im Harz ein reduziertes Schälrisiko an flachen Standorten (Abb. 5.7), ebenso jedoch an ganz steilen. Im mittleren Hangneigungsbereich verschiebt sich das Chancenverhältnis geschälter zu ungeschälter Bäume mit zunehmender Hangneigung hin zu mehr Neuschäle. Ab einem Wert von etwa $50 \%$ Steigung kehrt sich diese Tendenz dann aber wieder um. Im Solling zeigen sich die Chancenverhältnisse der Klassen ähnlich indifferent (Abb. 5.8). Doch liegen die geringen Abweichungen in den niedrigen Klassen noch näher am Mittelwert 


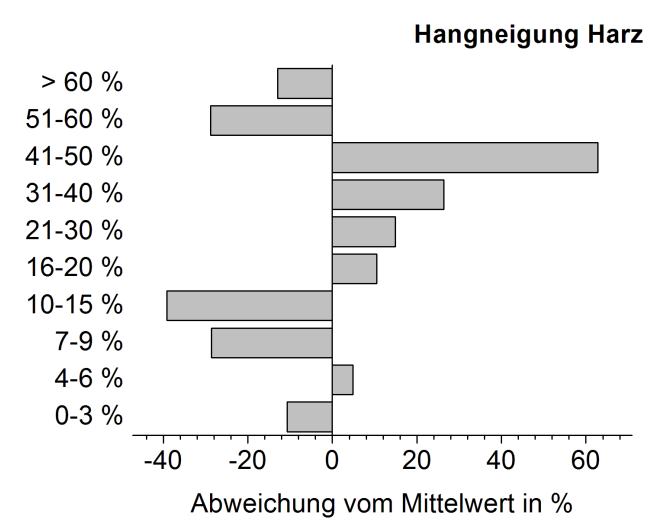

Abbildung 5.7: Relative Abweichung der mittleren Schälprozente einzelner Hangneigungssklassen vom Gesamtschälprozent im Harz; $\bar{x}=4,25 \%, n=773$

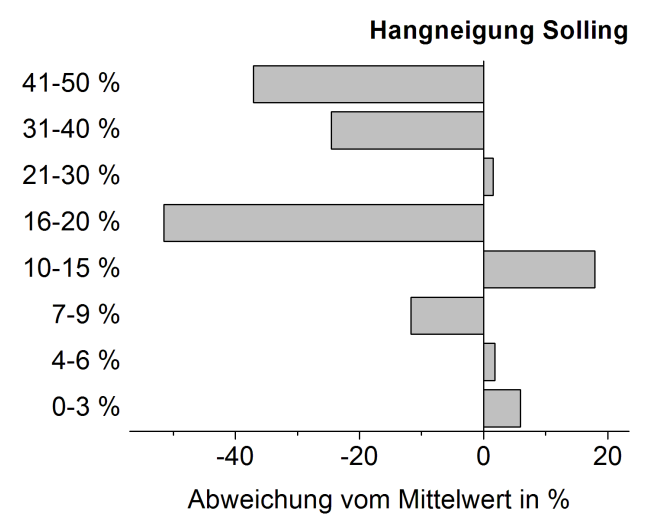

Abbildung 5.8: Relative Abweichung der mittleren Schälprozente einzelner Hangneigungssklassen vom Gesamtschälprozent im Solling; $\bar{x}=2,95 \%, n=891$

als im Harz. Auch hier lässt sich noch am ehesten in den steileren Lagen ein geringeres Neuschälrisko erkennen. Im Vergleich zum Harz sinkt die Schälwahrscheinlichkeit hier jedoch bereits bei geringeren Neigungsprozenten.

\subsubsection{Exposition}

Hinsichtlich der Ausrichtung der Standorte am Hang lassen die Expositionsklassen im Harz keine Aussagen zum erwarteten Chancenverhältnis zu (Abb. 5.9). Beginnend mit nördlicher Himmelsrichtung weichen die Chancenverhältnisse der einzelnen Klassen abwechselnd nach oben und unten vom Gesamtmittelwert ab. Im Solling zeigen sich erhöhte Werte an Hängen, die in Richtung Nord bis Ost orientiert sind (Abb. 5.10). Einzig die nach Südwesten ausgerichtete Klasse nimmt sich aus der Gruppe der restlichen Klassen mit eben dieser Tendenz aus.

\subsubsection{Exponiertheit}

Bei der Einteilung der Exponiertheitswerte in sechs Klassen weist die Klasse der geringsten Werte (0 bis 6) im Harz eine deutlich erhöhte Schälwahrscheinlichkeit gegenüber dem Durchschnitt auf (Abb. 5.11). Dieser Verschiebung der Chancenverhältnisse an schwach exponierten Standorten lässt sich im Solling (Abb. 5.12) nicht beobachten. Dort ist es die zweite Klasse, mit Exponiertheitswerten von 7 bis 12, die sich vom Rest der Klassen abhebt - hier jedoch durch eine Veränderung hin zu ei- 


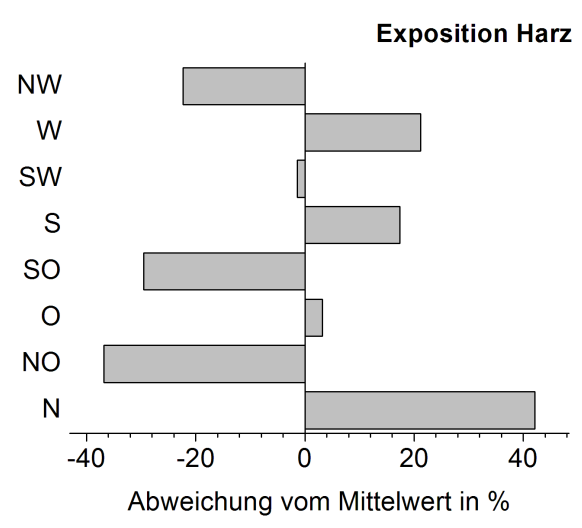

Abbildung 5.9: Relative Abweichung der mittleren Schälprozente einzelner Expositionsklassen vom Gesamtschälprozent im Harz; $\bar{x}=4,25 \%, n=773$

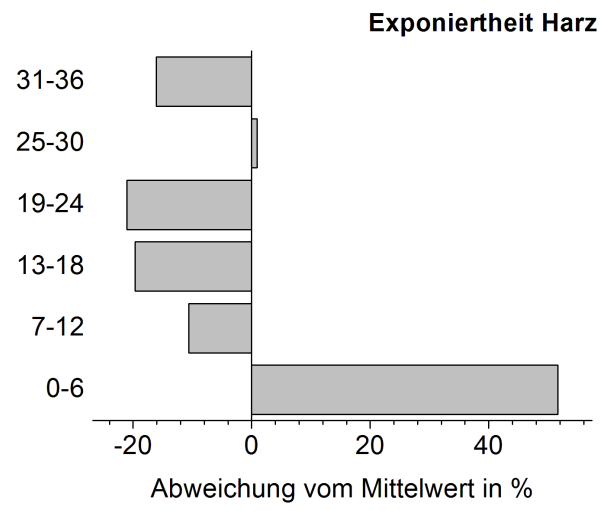

Abbildung 5.11: Relative Abweichung der mittleren Schälprozente einzelner Exponiertheitsklassen vom Gesamtschälprozent im Harz $; \bar{x}=4,25 \%, n=773$

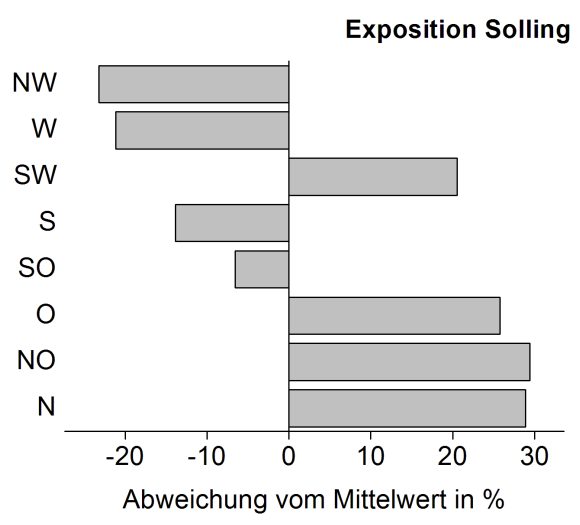

Abbildung 5.10: Relative Abweichung der mittleren Schälprozente einzelner Expositionsklassen vom Gesamtschälprozent im Solling; $\bar{x}=2,95 \%, n=891$

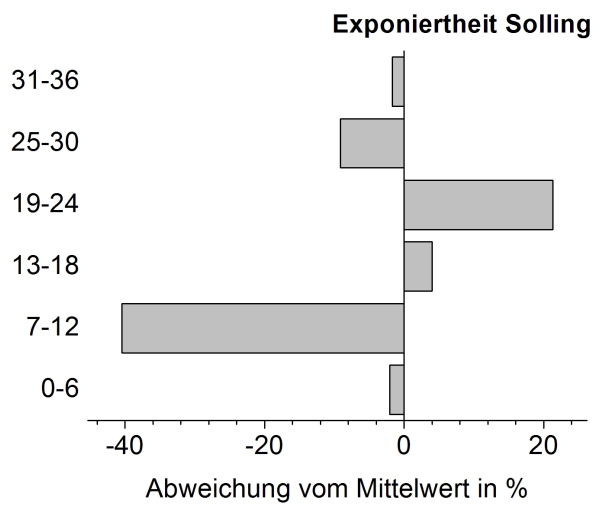

Abbildung 5.12: Relative Abweichung der mittleren Schälprozente einzelner Exponiertheitsklassen vom Gesamtschälprozent im Solling; $\bar{x}=2,95 \%, n=891$

nem geringeren Schälrisiko. Ähnlich wie im Harz pendeln sich im Solling alle anderen Expositionsklassen nahe dem Mittelwert ein.

\subsubsection{Strahlung}

Im Harz ergeben sich für die Klassen 0 bis 2 mit sehr geringen Strahlungswerten starke Abweichungen der Chancenverhältnisse vom Mittelwert um bis zu $100 \%$ zugunsten des ungeschälten Anteils (Abb. 5.13). Die unteren drei Klassen sind mit insgesamt 522 Stichproben jedoch nur schwach besetzt. Alle anderen Klassenwerte schwanken mit einer geringeren Amplitude um den mittleren Schälquotienten, wobei für die mitt- 


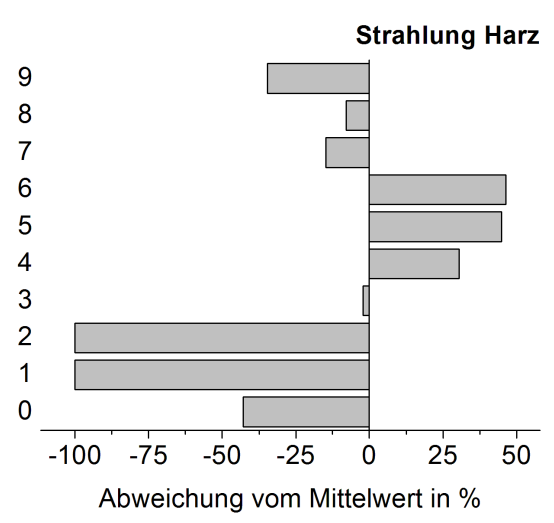

Abbildung 5.13: Relative Abweichung der mittleren Schälprozente einzelner Strahlungssklassen vom Gesamtschälprozent im Harz; $\bar{x}=4,25 \%, n=773$

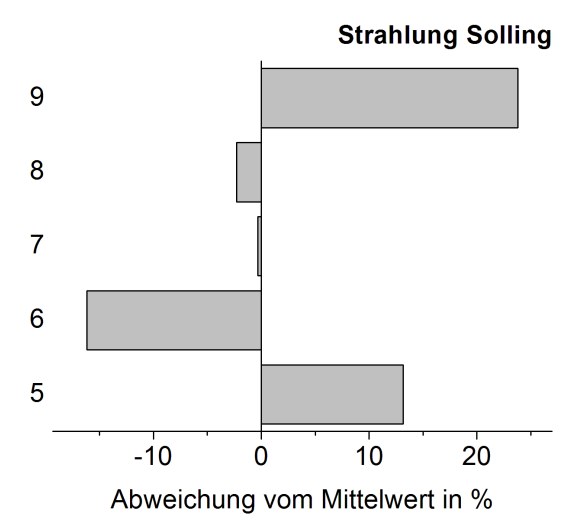

Abbildung 5.14: Relative Abweichung der mittleren Schälprozente einzelner Strahlungssklassen vom Gesamtschälprozent im Solling; $\bar{x}=2,95 \%, n=891$

leren Strahlungsklassen ein etwas höheres Schälrisiko ausgewiesen wird und für die höchsten wieder ein geringeres. Im Solling sind die Strahlungsklassen 0 bis 4 nicht besetzt (Abb. 5.14). Das sich darstellende Bild der Klassen 5 bis 9 ist weitestgehend indifferent. Die Abweichungen der Chancenverhältnisse bleiben in ihrer Ausprägung insgesamt hinter denen im Harz zurück. Der höchste Wert wird in der Klasse 9 erreicht, wo die Schälwahrscheinlichkeit um $25 \%$ vom mittleren Verhältnis abweicht.

\subsubsection{Bestandesalter}

Bei der Klassifizierung des Bestandesalters zeigt sich im Harz eine klare Tendenz (Abb. 5.15). Bestände jüngeren Alters weisen ein deutlich erhöhtes Schälrisiko gegenüber der mittleren Wahrscheinlichkeit auf. In den Klassen ansteigenden Bestandesalters fällt dieses Risiko dann zunächst abrupt und anschließend kontinuierlich ab. Auch im Solling zeichnen die Abweichungen der Chancenverhältnisse vom Mittelwert ein vergleichbares Bild (Abb. 5.16). Die dortige positive Verschiebung in der jüngsten Altersklasse fällt allerdings gegenüber dem Harz geringer aus und die negative Abweichung in der ältesten Klasse nimmt etwas höhere Werte an.

\subsubsection{Baumartenzahl}

Die nach der vorhandenen Baumartenzahl klassifizierten Punkte zeigen weder im Harz noch im Solling eine klare Tendenz in ihrem jeweiligen Chancenverhältnis geschälter 


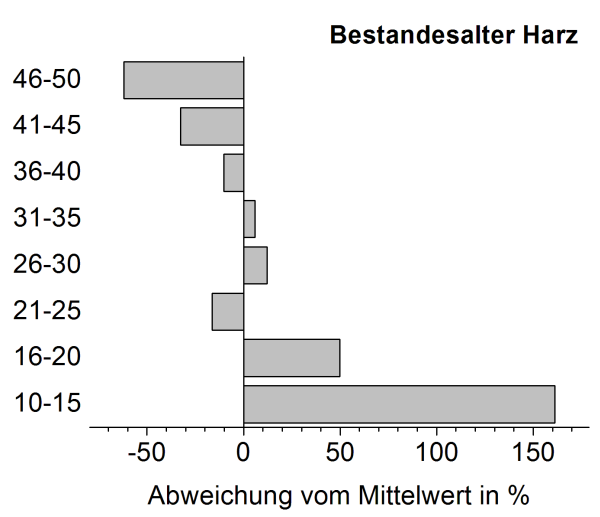

Abbildung 5.15: Relative Abweichung der mittleren Schälprozente einzelner Klassen des Bestandesalters vom Gesamtschälprozent im Harz; $\bar{x}=4,25 \%, n=773$

Baumarten Harz

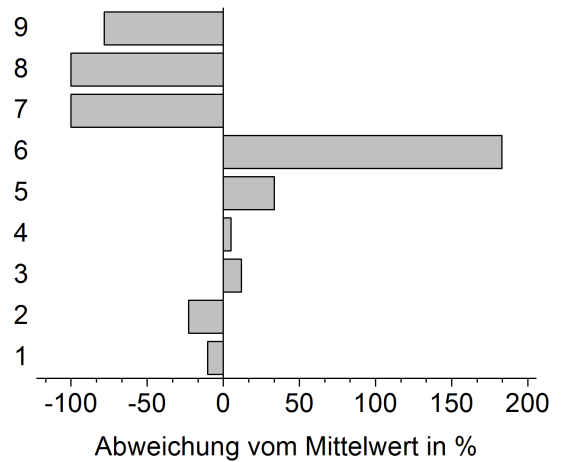

Abbildung 5.17: Relative Abweichung der mittleren Schälprozente einzelner Klassen der Baumartenzahl vom Gesamtschälprozent im Harz; $\bar{x}=4,25 \%, n=773$

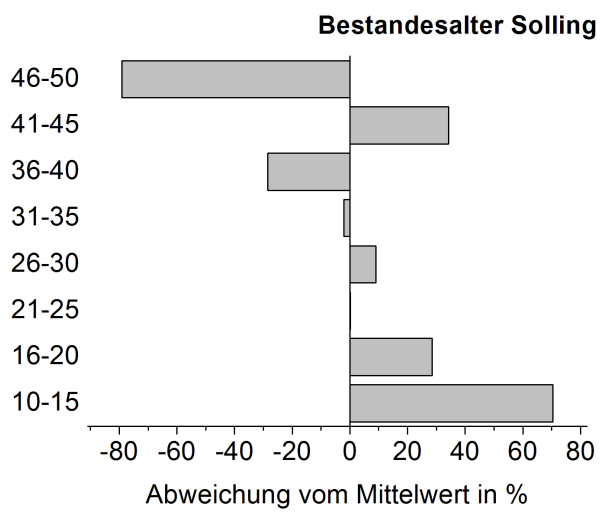

Abbildung 5.16: Relative Abweichung der mittleren Schälprozente einzelner Klassen des Bestandesalters vom Gesamtschälprozent im Solling; $\bar{x}=2,95 \%, n=891$

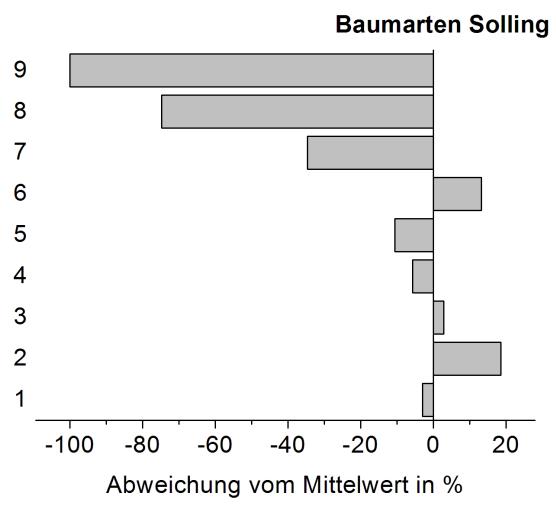

Abbildung 5.18: Relative Abweichung der mittleren Schälprozente einzelner Klassen der Baumartenzahl vom Gesamtschälprozent im Solling; $\bar{x}=2,95 \%, n=891$

zu ungeschälter Einheiten (Abb. 5.17 und 5.18). In den Klassen 1 bis 5 (im Solling bis Klasse 6) schwanken die Ergebnisse um den Gesamtmittelwert. Den extremen Abweichungen in den höheren Klassen liegt eine nur sehr geringe Anzahl an Datensätzen zugrunde. In ihrer Ausprägung zeigen diese Klassen in beiden Untersuchungsgebieten eine starke Verschiebung der Chancenverhältnisse hin zu einem geringeren Schälrisiko auf. 


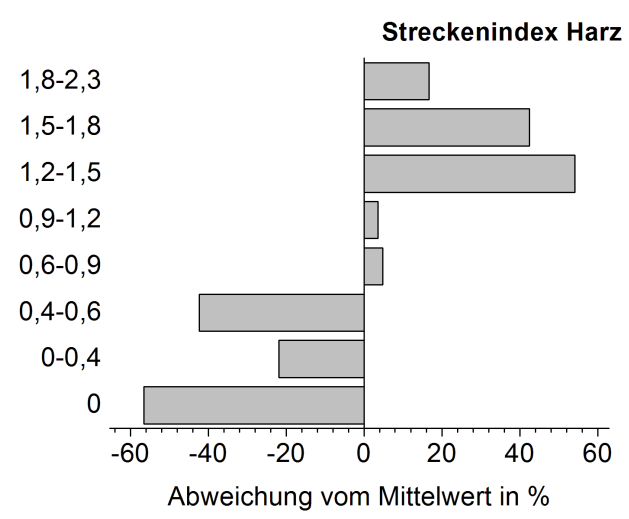

Abbildung 5.19: Relative Abweichung der mittleren Schälprozente einzelner Klassen des Jagdstreckenindizes vom Gesamtschälprozent im Harz; $\bar{x}=4,25 \%$, $n=733$

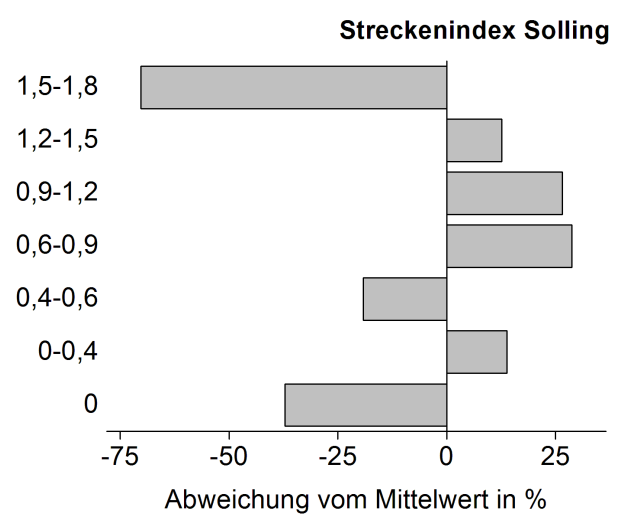

Abbildung 5.20: Relative Abweichung der mittleren Schälprozente einzelner Klassen des Jagdstreckenindizes vom Gesamtschälprozent im Solling; $\bar{x}=2,95 \%$, $n=891$

\subsubsection{Jagdstrecke}

Im Harz steigt die Wahrscheinlichkeit von Neuschäle mit Zunahme der Rotwildstrecke an (Abb. 5.19). Die Verschiebung der Chancenverhältnisse vom Mittelwert ist in beide Richtungen in etwa gleich stark ausgeprägt. In den höchsten Klassen verringert sich die Abweichung dann wieder etwas. Im Solling verhalten sich die Abweichungen tendenziell ähnlich, doch kehrt sich der Trend bei der Klasse mit dem höchsten Abschuss um (Abb. 5.20). Dort wird das geringste Schälrisiko ausgegeben. Der Klasse liegt jedoch ein etwas geringerer Datenumfang zugrunde.

\subsubsection{Wegeklasse}

Die Einteilung in unterschiedlich stark frequentierte Wanderwege im Harz zeigt für die drei ausgewiesenen Klassen unterschiedliche Chancenverhältnisse der Schäle (Abb. 5.21). Während die Schälwahrscheinlichkeit in der Klasse der mutmaßlich geringsten Frequentierung durch Wanderer über der mittleren Wahrscheinlichkeit liegt, weisen die jeweils höheren Klassen geringere Schälrisiken auf. Der Effekt der höchsten Klasse (2) ist dabei nochmals größer als bei der Klasse 1 . 


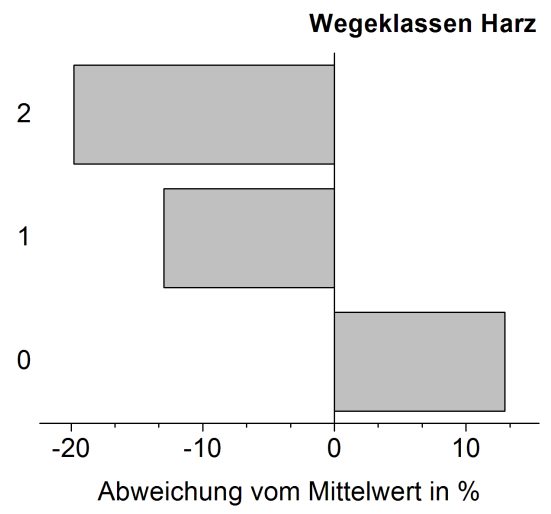

Abbildung 5.21: Relative Abweichung der mittleren Schälprozente einzelner Wegeklassen vom Gesamtschälprozent im Harz; $\bar{x}=4,45 \%, n=714$

\subsection{Zusammenfassung}

Im Harz besteht ein deutlich höheres Risiko für Neuschäle im Stichprobenpunkt, wenn der Anteil der vorgeschälten Bäume etwa 2/3 der Stichprobe erreicht. Weisen sämtliche Bäume Altschälschäden auf, verschiebt sich das lokale Chancenverhältnis im Vergleich zum Gesamtdurchschnitt um etwa 100 \%. Im Solling besteht eine vergleichbare Tendenz. Dort fällt vor allem das stark reduzierte Neuschälrisiko auf, wenn bisher keine Vorschäle am Punkt vorhanden war. In beiden Untersuchungsgebieten verschiebt sich das Chancenverhältnis an Orten höherer Kontinuität hin zu mehr Neuschäle.

Bei den geländebedingten Faktoren zeigt die Untersuchung nach der Höhenlage in keinem Gebiet ein klares Bild. An Standorten mit schwacher Hangneigung liegt das Schälrisiko unter dem Durchschnitt, gleiches gilt für sehr steile Standorte. Für die Exposition ergibt sich im Harz keine Tendenz, im Solling zeigen sich erhöhte Werte in Nord- bis Ostrichtung. Während eine geringe Exponiertheit im Gelände im Harz zu erhöhten Chancenverhältnissen führt, zeigen sich die Ergebnisse im Solling gegenüber diesem Faktor als indifferent. Bei sehr geringen Strahlungswerten wird im Harz ein stark reduziertes Schälrisiko festgestellt. Diese Parameterklassen sind im Solling nicht durch Stichprobenpunkte besetzt. Das sich darstellende Bild der höheren Strahlungsklassen bleibt dort ohne Tendenz.

Bei der Betrachtung des Bestandesalters weisen jüngere Bestände in beiden Gebieten ein deutlich erhöhtes Schälrisiko gegenüber der mittleren Wahrscheinlichkeit auf. Bei der Klassifizierung nach Baumartenzahlen zeigt sich hingegen weder im Harz noch im 
Solling eine Tendenz der Chancenverhältnisse geschälter zu ungeschälter Einheiten. Im Harz steigt die Wahrscheinlichkeit von Neuschäle mit Zunahme der Rotwildstrecke an. Im Solling verhalten sich die Abweichungen tendenziell ähnlich, doch kehrt sich der Trend bei der Klasse mit dem höchsten Abschuss um. Dort wird das geringste Schälrisiko ausgegeben. Die Wegeklassifizierung im Harz zeigt eine klare Tendenz. Die mutmaßlich höhere Frequentierung der Wege durch den Menschen geht dort mit einem verringerten Chancenverhältnis der Neuschäle einher. Im Solling steht dieser Parameter nicht für die Untersuchung zur Verfügung. 


\section{Binäre logistische Regressionsmodelle}

\subsection{Ergebnisse}

Bei den folgenden Untersuchungsergebnissen handelt es sich um die Auswertung des Datensets der homogenen Stichprobenpunkte im Harz, welches in den Voruntersuchungen weitestgehend charakterisiert und vom heterogenen Datenset abgegrenzt wurde. Wie in 2.4.2 auf Seite 29 beschrieben, sind in die hier aufgelisteten Modelle zunächst sämtliche Parameter, welche als erklärende Variablen zur Verfügung stehen, eingegangen. Durch die rückwärts gerichtete, schrittweise Selektion enthalten die nachstehenden Tabellen ausschließlich solche Variablen, deren Effekt im Modell als signifikant $(p<0,05)$ eingestuft wurde. Bei der Darstellung der Ergebnisse soll jeweils zu Beginn ein Blick auf die Anpassungsgüte der Modelle sowie auf deren Klassifizierungspotential gerichtet werden, bevor im Anschluss die Effektparameter der Variablen einzeln aufgeführt werden. Hierzu sind die Werte von Anpassung und Klassifizierung der Gebiete vorab für alle drei Inventurperioden gemeinsam in Tabelle 6.1 dargestellt.

Tabelle 6.1: Anpassungsgüte und Klassifizierungen der Modelle der Harzinventuren in der Übersicht

\begin{tabular}{lrrrcrrr}
\hline Gebiet & \multicolumn{2}{c}{ Hosmer-Lemeshow-Test } & Nagelkerkes & \multicolumn{3}{c}{ Anteil korrekter Klassifizierungen } \\
\& Jahr & Chi-Quadrat & df & Sig. & R-Quadrat & ungeschält & geschält & gesamt \\
\hline \multirow{2}{*}{ Harz 2002 } & 5,547 & 8 & 0,698 & 0,311 & $82,5 \%$ & $55,8 \%$ & $72,4 \%$ \\
Harz 2004 & 9,871 & 8 & 0,274 & 0,210 & $90,2 \%$ & $38,8 \%$ & $73,9 \%$ \\
Harz 2006 & 7,180 & 8 & 0,517 & 0,135 & $91,3 \%$ & $22,6 \%$ & $69,7 \%$ \\
\hline
\end{tabular}




\subsubsection{Inventurjahre 2002, 2004 und 2006 im Harz}

Über die berechneten Werte des Hosmer-Lemeshow-Tests (Tab. 6.1) zeigt sich, dass das vorliegende Modell in adäquater Weise an die Daten angepasst ist und dass diese Feststellung als signifikant gelten kann (im Zusammenhang mit diesem Test weisen Werte von Sig. > 0, 05 Signifikanz nach (Hosmer \& Lemeshow 2000)). Zudem weist der Wert von 0,311 des Nagelkerkes-R-Quadrats auf ein breites Erklärungspotential der Varianz des Modells hin. Nach LiTz 2000 können bereits Werte ab > 0,2 als gut bewertet werden, sofern diesen logistische Regressionsmodelle zugrunde liegen. Bei der Klassifizierung der 0-1 codierten abhängigen Variablen (vgl. 2.4.2, S. 29) erreicht das Modell einen Anteil korrekt zugewiesener Plots von 72,4 \% an der Gesamtstichprobe. Neben dem Wert von $82,5 \%$ bei den nicht geschälten Stichprobenpunkten ${ }^{1}$ sagt das Modell mit 55,8 \% vor allem bei den Inventurpunkten mit Neuschälvorkommen für einen erheblichen Anteil die jeweilige Klassenzugehörigkeit korrekt voraus.

In Tabelle 6.2 sind die für die Anpassungsgüte relevanten Variablen aufgeführt. Neben dem metrisch skalierten Faktor des Altschälvorkommens ist mit dem Expositionswert auch eine ordinal skalierte unabhängige Variable im Modell verblieben. Die zugehörigen Klassen der Exposition sind eingerückt unterhalb des Parameters dargestellt und können somit einzeln bewertet werden. Die als Referenzklasse nicht separat aufgeführte Ausprägung „Nord“ dient hierbei für jede Klasse als Bezugsgröße. Da nicht alle Klassen einer ordinalskalierten Variable obligatorisch signifikant sind, wurden jene mit signifikantem Einfluss in der Tabelle mit Sternchen gekennzeichnet. Hinsichtlich ihres Effektes auf die Schälwahrscheinlichkeit erweisen sich die Werte nordöstlicher und südwestlicher Expositionensklassen also im vorliegenden Modell als signifikant anders als solche der nördlichen Exposition. In beiden Fällen ist das Neuschälrisiko gegenüber nach Norden ausgerichteter Standorte als geringer zu bewerten. Die Klasse Nordost zeigt hierbei mit $e^{\beta}=0,035$ den extremsten Wert, doch auch das sechsmal geringe Schälrisiko der Klasse Südwest distanziert sich deutlich vom Referenzwert. Die Werte beider Klassen bleiben auch im 95 \% Vertrauensbereich in ihrer Wirkungsrichtung konstant.

Gleiches gilt für die Variable der im Stichprobenpunkt vorhandenen Altschälschäden. Der zugehörige Effektparameter $e^{\circledR}$ indiziert hier eine Zunahme des Schälrisikos um jeweils $22 \%$, wenn ein weiterer Baum innerhalb des Stichprobenpunktes alte Schälschä-

\footnotetext{
${ }^{1}$ Bei der Verwendung der Begriffe „ungeschält“ oder „nicht geschält“ ist in diesem Zusammenhang stets nur von aktueller Winter-Neuschäle im Plot die Rede. Alte Schälschädigungen bleiben hierbei natürlich unberücksichtigt.
} 
Tabelle 6.2: Erklärende Variablen des binär logistischen Regressionsmodells Harz 2002; Referenzklasse der Exposition: Nord

\begin{tabular}{lrrrrrr}
\hline Variable / Klasse & $B$ & $s_{\bar{x}}$ & Wald & $p$ & $e^{\beta}$ & $K I e^{\beta}$ \\
\hline Altschäle & 0,196 & 0,036 & 28,802 & $<0,001$ & 1,216 & $1,132-1,306$ \\
Exposition & & & 19,170 & 0,008 & & \\
$\quad$ NO** $^{* *}$ & $-3,361$ & 1,168 & 8,273 & 0,004 & 0,035 & $0,004-0,343$ \\
O & $-1,428$ & 0,822 & 3,017 & 0,082 & 0,240 & $0,048-1,201$ \\
SO & $-1,156$ & 0,634 & 3,324 & 0,068 & 0,315 & $0,091-1,091$ \\
S & 0,073 & 0,588 & 0,015 & 0,901 & 1,076 & $0,340-3,407$ \\
SW** & $-1,795$ & 0,680 & 6,967 & 0,008 & 0,166 & $0,044-0,630$ \\
W & $-0,300$ & 0,597 & 0,253 & 0,615 & 0,741 & $0,230-2,389$ \\
NW & $-0,986$ & 0,641 & 2,369 & 0,124 & 0,373 & $0,106-1,309$ \\
Konstante & $-1,631$ & 0,554 & 8,680 & 0,003 & 0,196 & \\
\hline
\end{tabular}

$B=$ Regressionskoeffizient $B, s_{\bar{x}}=$ Standardfehler, Wald $=$ Wert der Waldstatistik, $p=$ Signifikanzwert des $W$ ald-Wertes, $e^{\beta}=$ Odds Ratio, $K I=95 \%$ Konfidenzintervall von $e^{B}$

digungen aufweist. Dieser in seiner Effektgröße sehr ausgeprägte Faktor dominiert auch im Vergleich der Wald-Werte deutlich vor dem Einfluss der Exposition. Alle anderen dem Modell zur Verfügung gestellten Parameter sind nicht als erklärende Variablen im Resultatmodell verblieben.

Für das Modell des Jahres 2004 wird in Tabelle 6.1 ein deutlich geringerer Signifikanzwert im Hosmer-Lemeshow-Test der Modellanpassung als im Modell der Vorperiode aufgeführt $(0,274$ gegenüber 0,698$)$. Auch der R-Quadrat-Wert bleibt mit 0,210 hinter dem vorherigen zurück. Entsprechend schwächer fällt auch das Ergebnis der Klassifizierungsleistung aus. Zwar konnten insgesamt sogar 73,9 \% der Stichprobenpunkte korrekt zugeordnet werden, doch ergibt sich diese leichte Steigerung ausschließlich aus dem hohen Anteil von 90,2 \% richtig bestimmter ungeschälter Plots. Der für die Prognose weitaus wichtigere Wert der korrekt klassifizierten geschälten Inventurpunkte fällt indes von ehemals 55,8\% auf nunmehr 38,8 \% zurück.

Zusätzlich zu den im Jahr 2002 selektierten Variablen verbleibt im betrachteten Regressionsmodell der Faktor der Baumartenzahl der weiteren Umgebung als erklärende Variable im finalen Modell übrig (Tab. 6.3). Mit jeder weiteren Baumart in der den Inventurpunkt umgebenden Abteilung steigt die Wahrscheinlichkeit der Neuschäle im Plot demnach um 16 \%. Im Vergleich zur Vorperiode nimmt der erneut signifikante Ef- 
Tabelle 6.3: Erklärende Variablen des binär logistischen Regressionsmodells Harz 2004; Referenzklasse der Exposition: Nord

\begin{tabular}{lrrrrrr}
\hline Variable / Klasse & $B$ & $s_{\bar{x}}$ & Wald & $p$ & $e^{\beta}$ & $K I e^{\beta}$ \\
\hline Altschäle & 0,116 & 0,028 & 17,633 & $<0,001$ & 1,123 & $1,064-1,186$ \\
Exposition & & & 16,857 & 0,018 & & \\
$\quad$ NO** & $-1,651$ & 0,706 & 5,461 & 0,019 & 0,192 & $0,048-0,766$ \\
O** $^{* *}$ & $-1,316$ & 0,637 & 4,263 & 0,039 & 0,268 & $0,077-0,935$ \\
SO & $-1,076$ & 0,558 & 3,719 & 0,054 & 0,341 & $0,114-1,018$ \\
S & $-0,378$ & 0,527 & 0,515 & 0,473 & 0,685 & $0,244-1,924$ \\
SW** & $-1,147$ & 0,564 & 4,133 & 0,042 & 0,318 & $0,105-0,960$ \\
W** & $-1,957$ & 0,586 & 11,166 & 0,001 & 0,141 & $0,045-0,445$ \\
$\quad$ NW** & $-1,317$ & 0,560 & 5,535 & 0,019 & 0,268 & $0,089-0,803$ \\
BAzahlAbt & 0,147 & 0,058 & 6,471 & 0,011 & 1,158 & $1,034-1,297$ \\
Konstante & $-1,447$ & 0,535 & 7,331 & 0,007 & 0,235 & \\
\hline
\end{tabular}

$B=$ Regressionskoeffizient $B, s_{\bar{x}}=$ Standardfehler, $W$ ald $=$ Wert der Waldstatistik, $p=$ Signifikanzwert des $W$ ald-Wertes, $e^{\beta}=$ Odds Ratio, $K I=95 \%$ Konfidenzintervall von

fekt des Altschälvorkommens etwas in seiner Stärke ab und weist einen Risikoanstieg um $12 \%$ bei jeder weiteren Einheit (also altgeschältem Baum im Plot) aus. Bei der Exposition des Standortes als Faktor erhöht sich in der Periode 2004 die Anzahl der signifikant vom Referenzwert Nord abweichenden Klassen. Neben den Richtungklassen Nordost und Südwest distanzieren sich nun auch die Ausprägungen Ost-, West-, und Nordwestexposition in ihrem jeweiligen Effekt von nach Norden ausgerichteten Standorten. Alle fünf Hangrichtungsklassen weisen hierbei eine statistisch geringere Wahrscheinlichkeit eines Neuschälvorkommens auf. Mit einem Quotienten von 1:0,141 kann das Schälrisiko für Bestände hiernach an Westhängen als etwa siebenmal geringer als an Nordhängen bezeichnet werden. Dieser Wert spiegelt den ausgeprägtesten Unterschied innerhalb der Expositionsklassen wider. Im Nordosten kann von einem etwa fünfmal geringeren Risiko gesprochen werden und im Osten und Nordwesten jeweils von einem um das knapp Vierfache verringerten Wert. Südwestlich exponierte Inventurpunkte weisen immerhin noch eine etwa dreimal niedrigere Schälgefährdung auf als nördlich exponierte Inventurpunkte. Insgesamt erreicht die Variable Exposition einen fast ebenso hohen Wald-Wert wie die Variable Altschäle. 
Tabelle 6.4: Erklärende Variablen des binär logistischen Regressionsmodells Harz 2006

\begin{tabular}{lrrrrrr}
\hline Variable / Klasse & $B$ & $s_{\bar{x}}$ & Wald & $p$ & $e^{\mathrm{B}}$ & $K I e^{\mathrm{B}}$ \\
\hline Altschäle & 0,110 & 0,026 & 17,896 & $<0,001$ & 1,116 & $1,061-1,174$ \\
BAzahlAbt & 0,134 & 0,057 & 5,544 & 0,019 & 1,144 & $1,023-1,279$ \\
Konstante & $-2,443$ & 0,396 & 38,028 & $<0,001$ & 0,087 & \\
\hline
\end{tabular}

$B=$ Regressionskoeffizient $B, s_{\bar{x}}=$ Standardfehler, Wald $=$ Wert der Waldstatistik, $p=$ Signifikanzwert des $W$ ald-Wertes, $e^{\beta}=$ Odds Ratio, $K I=95 \%$ Konfidenzintervall von $e^{\mathrm{B}}$

Das Regressionsmodell für die Periode 2006 erweist sich als hinreichend an die Daten angepasst (Tab. 6.1), kann mit seinem R-Quadratwert von 0,135 nach Nagelkerkes jedoch nicht das Erklärungspotential der beiden Modelle vorangegangener Perioden erreichen. So werden noch 69,7 \% der Stichprobenpunkte richtig klassifiziert, doch sorgt erneut ein erhöhter Anteil korrekt als nicht geschält eingestufter Plots (91,3 \%) für das Halten dieses Niveaus. Mit lediglich 22,6 \% korrekt als neugeschält klassifizierter Plots bleibt deren Anteil deutlich hinter dem der Modelle vorangegangener Jahre zurück.

Hinsichtlich der im Regressionsmodell als signifikante Einflussfaktoren verbliebenen unabhängigen Variablen zeigt sich die Information über vorhandene Altschäle erneut als effektbestimmender Faktor (Tab. 6.4). Mit der Zunahme des Schälrisikos um 12 \% bei Erhöhung um eine Einheit des Faktors liegt dieser Wert auf gleichem Niveau wie in der vorangegangenen Periode. Einflüsse der Exposition des Standortes auf die Wahrscheinlichkeit eines Neuschälereignisses können im vorliegenden Modell hingegen nicht mehr festgestellt werden. Die Anzahl der Baumarten verbleibt, auf der Ebene der weiteren Umgebung berechnet, jedoch erneut als erklärende Variable im Modell. Mit 14 \% Risikozunahme je Erhöhung um eine Einheit fällt ihre Einflussgröße im Vergleich zum Inventurjahr 2004 ähnlich aus.

\subsubsection{Inventurjahre 2002, 2003 und 2006 im Solling}

Wie bereits im Ergebnisteil der Regressionsrechnungen für den Harz sollen auch hier die Güte der Modellanpassung und der Klassifizierung zu Beginn präsentiert werden. Die inhaltlichen Ergebnisse der für die einzelnen Perioden gerechneten Modelle werden dann wiederum getrennt voneinander dargestellt. 
6. Binäre logistische Regressionsmodelle

Tabelle 6.5: Anpassungsgüte und Klassifizierungen der Modelle der Sollinginventuren in der Übersicht

\begin{tabular}{lrrrcrrr}
\hline Gebiet & \multicolumn{3}{c}{ Hosmer-Lemeshow-Test } & Nagelkerkes & \multicolumn{3}{c}{ Anteil korrekter Klassifizierungen } \\
\& Jahr & Chi-Quadrat & df & Sig. & R-Quadrat & ungeschält & geschält & gesamt \\
\hline Solling 2002 & 2,625 & 8 & 0,956 & 0,051 & $95,5 \%$ & $10,5 \%$ & $64,1 \%$ \\
Solling 2003 & 15,206 & 8 & 0,055 & 0,030 & $100,0 \%$ & $0,0 \%$ & $73,2 \%$ \\
Solling 2006 & 14,914 & 8 & 0,061 & 0,034 & $100,0 \%$ & $1,9 \%$ & $78,7 \%$ \\
\hline
\end{tabular}

Die Berechnung der in Periode 2002 im Solling aufgenommenen Inventurdaten führt zu einem Modell, dessen Erklärungspotential mit einem R-Quadratwert von 0,051 zwar gering bleibt, sich jedoch trotzdem signifikant vom Zufall unterscheidet (Tab. 6.5). Es werden zwei Variablen mit vergleichbar niedrigem Wald-Wert vom Modell als signifikante Effektparameter ausgewiesen (Tab.6.6). Demnach sinkt die Schälwahrscheinlichkeit an einem Inventurpunkt um etwa $13 \%$, wenn sich die mittlere Bestandesgröße um eine Einheit erhöht. Die Berechnung des Parameters bezieht sich hierbei auf die Ebene der Abteilung. Im Effekt noch etwas stärker wirkt sich die Baumartenzahl gleicher Betrachtungsebene auf das mutmaßliche Schälrisiko aus. Entgegen dem für die Jahre 2004 und 2006 im Harz festgestellten Zusammenhang zwischen Baumartenzahl und Schälwahrscheinlichkeit (Tab. 6.3 und 6.4) wird hier der auf die Flächengröße bezogene Baumarten-Index als erklärende Variable ausgewiesen. Die Bezugseinheit ist in beiden Fällen die Abteilung, doch ergibt sich im vorliegenden Fall eine Aussage hinsichtlich der relativen Anzahl an Baumarten je 10 Hektar Abteilungsfläche, welche entsprechend auch als Dezimalzahl vorliegen kann. Mit der Zunahme dieses Index um eine Einheit verringert sich das Schälrisiko also um 25 \%. Erwähnt sei an dieser Stelle noch, dass der Faktor Altschäle in diesem Modell nicht als erklärende Variable ausgewiesen wird.

Im Jahr 2003 wird das notwendige Signifikanzniveau des Rechenmodells mit einem Wert von 0,055 gerade noch erreicht. Der Wert des R-Quadrats nach Nagelkerkes sinkt gegenüber dem Wert des Vorjahres noch einmal ab. Es werden so gut wie keine Varianzen durch das Modell erklärt (Tab. 6.5). Widergespiegelt wird dieser Befund durch die Auflistung der Klassifizierungen. Nicht geschälte Inventurpunkte werden zu $100 \%$ korrekt klassifiziert, doch kann kein einziger tatsächlich geschälter Punkt als solcher durch das Modell erkannt werden. Das Gesamtergebnis, nach welchem ein Anteil von 73,2 \% 
der Stichprobe erklärt wird, täuscht also über das schlechte Ergebnis im Sinne einer Risikovorhersage hinweg. Als erklärende Variable ist lediglich das Altschälvorkommen im Stichprobenpunkt im Modell verblieben (Tab. 6.7). Hier zeigt sich ein mittlerer Anstieg des Risikos um knapp 7 \% je weiterem vorgeschälten Baum im Plot. Alle anderen Parameter wurden nicht für eine Verbesserung in der Modellanpassung herangezogen.

Auch das dritte Regressionsmodell im Solling für die Inventur im Jahr 2006 zeigt keine guten Werte der Anpassung. Tabelle 6.5 weist neben einem niedrigen Signifikanzniveau auch einen geringen R-Quadrat-Wert aus. Wie im Modell für das Jahr 2003 werden die vorhandenen Ausprägungen der abhängigen Variablen kaum vom Modell erklärt. Wieder erreicht der Klassifizierungswert der nicht geschälten Inventurpunkte einen Anteil von $100 \%$ und erneut können geschädigte Punkte in kaum einem Fall erkannt werden. Als erklärende Variablen werden zwei Paramater eingesetzt, deren Effekte sich trotz inhaltlich ähnlicher Aussage kontrastieren. So zeigt die absolute Baumartenanzahl innerhalb der weiteren Umgebung ein negatives Verhältnis zur Wahrscheinlichkeit des Aufkommens frischer Schälschäden, während der relative Anteil der Baumartenzahl der weiteren Umgebung ein positives Verhältnis hierzu aufweist. Dort ergibt sich eine Zunahme des Schälrisikos um immerhin $48 \%$, wenn sich der Index um eine Einheit erhöht. Steigt hingegen die absolute Zahl der Baumarten in der betrachteten Abteilung, bedeutet dies eine Verringerung des Schälrisikos um 17 \%. Wie im Modell des Jahres 2002 wird auch hier die Information über Altschälschäden im Stichprobenpunkt nicht als erklärende Variable durch das Modell hinzugezogen.

Tabelle 6.6: Erklärende Variablen des binär logistischen Regressionsmodells Solling 2002

\begin{tabular}{lrrrrrr}
\hline Variable / Klasse & $B$ & $s_{\bar{x}}$ & Wald & $p$ & $e^{\beta}$ & $K I e^{\beta}$ \\
\hline mBestGrAbt & $-0,143$ & 0,055 & 6,727 & 0,009 & 0,867 & $0,778-0,966$ \\
BAzahlAbt 10ha & $-0,283$ & 0,108 & 6,892 & 0,009 & 0,753 & $0,610-0,931$ \\
Konstante & 0,974 & 0,499 & 3,813 & 0,051 & 2,648 & \\
\hline
\end{tabular}

$B=$ Regressionskoeffizient $B, s_{\bar{x}}=$ Standardfehler, Wald $=$ Wert der Waldstatistik, $p=$ Signifikanzwert des $W$ ald-Wertes, $e^{\beta}=$ Odds Ratio, $K I=95 \%$ Konfidenzintervall von $e^{B}$ 
6. Binäre logistische Regressionsmodelle

Tabelle 6.7: Erklärende Variablen des binär logistischen Regressionsmodells Solling 2003

\begin{tabular}{lrrrrrr}
\hline Variable / Klasse & $B$ & $s_{\bar{x}}$ & Wald & $p$ & $e^{\mathrm{B}}$ & $K I e^{\mathrm{\beta}}$ \\
\hline Altschäle & 0,064 & 0,026 & 5,928 & 0,015 & 1,066 & $1,013-1,122$ \\
Konstante & $-1,686$ & 0,319 & 27,886 & $<0,001$ & 0,185 & \\
\hline
\end{tabular}

$B=$ Regressionskoeffizient $B, s_{\bar{x}}=$ Standardfehler, Wald $=$ Wert der Waldstatistik, $p=$ Signifikanzwert des $W$ ald-Wertes, $e^{\beta}=$ Odds Ratio, $K I=95 \%$ Konfidenzintervall von $e^{\beta}$

Tabelle 6.8: Erklärende Variablen des binär logistischen Regressionsmodells Solling 2006

\begin{tabular}{lrrrrrr}
\hline Variable / Klasse & $B$ & $s_{\bar{x}}$ & Wald & $p$ & $e^{\mathrm{B}}$ & $K I e^{\mathrm{\beta}}$ \\
\hline BAzahlAbt & $-0,190$ & 0,090 & 4,426 & 0,035 & 0,827 & $0,693-0,987$ \\
BAzahlAbt10ha & 0,394 & 0,174 & 5,142 & 0,023 & 1,483 & $1,055-2,085$ \\
Konstante & $-1,248$ & 0,351 & 12,632 & $<0,001$ & 0,287 & \\
\hline
\end{tabular}

$B=$ Regressionskoeffizient $B, s_{\bar{x}}=$ Standardfehler, Wald $=$ Wert der Waldstatistik, $p=$ Signifikanzwert des $W$ ald-Wertes, $e^{\mathrm{B}}=$ Odds Ratio, $K I=95 \%$ Konfidenzintervall von $e^{\mathrm{B}}$

\subsubsection{Gesamtmodell}

Die Ergebnisse der binären logistischen Regressionsmodelle für Harz und Solling sind gemeinsam in Tabelle 6.9 aufgeführt. Angegeben sind die Effektstärke und Effektrichtung (Estimate $=$ positiv / negativ) der getesteten Parameter und das Signifikanzniveau als erklärende Variable im Modell. Hieraus ergeben sich im Detail folgende Aussagen (vgl. Tab. 6.9).

Bei der binären logistischen Regression wird der Parameter „Jahr“ lediglich im Modell für den Solling als Effektvariable ausgewiesen (hochsignifikant). Die Information über bereits vorhandene Altschälschäden im Stichprobenpunkt wird hingegen in beiden Modellen als positiv gerichteter Wert diagnostiziert (hochsignifikant). Von den vier getesteten geografischen Parametern verbleiben drei als erklärende Variablen im Harzmodell, während hierdurch im Solling keine Effekte festgestellt werden. Auch die für den Harz ausgewiesene, leichte Erhöhung des Schälrisikos bei zunehmender Hangneigung des Standortes ist nicht signifikant. Bei der Hangrichtung ergeben sich für alle klassifizierten Richtungen im vergleich zum Norden negative Effektwerte, die bis auf den 
Wert für die südliche Exposition als signifikant ausgewiesen werden. Die Effektstärke ist im Vergleich zu den anderen Modellvariablen hoch. Den höchsten Wert erreicht die nordöstliche, den niedrigsten Wert die nordwestliche Exposition. Beim potentiellen Strahlungsinput im Januar zeigt sich eine signifikante Zunahme des Schälrisikos bei steigenden Werten. Die Exponiertheit im Gelände wird in keinem der beiden Modelle als erklärende Variable verwendet.

Die Baumartenzahl innerhalb der Abteilung wird im Harz als positiv und im Solling als negativ wirksame Effektvariable im Modell belassen, wobei für den Harz ein höheres Signifikanzniveau angenommen wird. Die Einteilung der Forstwege in unterschiedlich stark frequentierte Wanderwege im Harz hat auf das Schälrisiko lokal zugeordneter Stichprobenpunkte keinen signifikanten Einfluss. Für das Modell im Solling stand dieser Parameter nicht zur Verfügung. Der über die Jagdstreckenlisten berechnete Rotwilddichteindex wird im Modell für den Solling als negativ wirkender Effekt ausgewiesen. Der Effekt ist in diesem Modell der deutlich stärkste Effekt. Im Harz wird der Parameter hingegen nicht als erklärende Variable durch das Modell herangezogen.

\subsection{Zusammenfassung}

Die für die Inventuren im Harz durchgeführten Regressionsmodelle variieren in ihrem Niveau der Modellanpassung und des Erklärungspotentials. Insbesondere der Anteil korrekt als geschält klassifizierter Daten nimmt im Vergleich der drei Inventuren über die Jahre ab. Auch die als Effektparameter ausgewiesenen Variablen unterscheiden sich teilweise zwischen den Modellen. Während die Information über Altschälvorkommen in allen drei Modellen als erklärende Variable hinzugezogen wird, werden Exposition und Baumartenzahl der Umgebung jeweils in zwei Modellen als Einflussgrößen genannt.

In den Regressionsmodellen für den Solling bleiben die Modellanpassung und das Erklärungspotential deutlich hinter den entsprechenden Werten der Harzmodelle zurück. Neben den Parametern der Altschälschäden und der Baumartenzahl wird hier auch die mittlere Bestandesgröße als Faktor in einem Modell angeführt. Im Gegensatz zu den Ergebnissen im Harz wird das Vorhandensein alter Schälschäden zudem nur in einem der drei Modelle als erklärende Variable belassen.

Bei der abschließenden Betrachtung über alle drei Inventurjahre hinweg zeigt sich die Differenzierung nach Jahren lediglich im Solling als signifikanter Effektparameter. 
Tabelle 6.9: Variablen im Gesamtmodell für Harz und Solling

\begin{tabular}{lcccc}
\hline & \multicolumn{2}{c}{ Harz $2002-04-06$} & \multicolumn{2}{c}{ Solling 2002-03-06 } \\
Parameter & Estimate & Sig & Estimate & Sig \\
\hline Jahr & & & $-1,81$ & $* * *$ \\
Altschäle & 1,33 & $* * *$ & 0,66 & $* * *$ \\
Hangneigung & 0,12 & & & \\
Hangrichtung (Nord) & & & & \\
$\quad$ NO & $-17,36$ & $* * *$ & & \\
O & $-10,09$ & $* *$ & & \\
SO & $-14,88$ & $* * *$ & & \\
S & $-5,25$ & & & \\
SW & $-14,58$ & $* * *$ & & \\
W & $-11,09$ & $* *$ & & \\
$\quad$ NW & $-8,08$ & $*$ & & \\
Januarsonne & 1,71 & $*$ & & \\
Exponiertheit & & & & \\
Baumartenzahl Abt & 1,08 & $* *$ & & \\
Baumartenzahl Ufl & & & & \\
Erholungszone & & & & \\
Rotwildindex & & & & \\
\hline
\end{tabular}

Alle Werte mit 10 multipliziert.

Maßgeblicher Faktor in beiden Gebieten ist wiederum die Variable der Altschälschäden. Von den vier getesteten geografischen Parametern werden im Harz die Hangrichtung und der potentielle Strahlungsinput als erklärende Variablen im Modell erkannt und deren Einfluss als signifikant ausgewiesen. Im Solling verbleibt hingegen keiner dieser Parameter im finalen Regressionsmodell. Für die Baumartenzahl werden ja nach Gebiet verschiedene Effektrichtungen angegeben. Die Information über die Frequentierung von Wanderwegen im Harz führt zu keiner Modellaussage. Bei der Einbeziehung der erzielten Rotwildstrecke ergeben sich im Harz ebenfalls keine Effekte, während dieser Faktor im Solling die stärkste Effektvariable darstellt und in negativer Richtung auf die abhängige Variable einwirkt. 


\section{Generalisierte Lineare Modelle}

\subsection{Ergebnisse}

Als Datengrundlage der folgenden Analyse dienen die im unteren (dem Kapitel 7 zugeordneten) Teil der Tabelle 2.4 unter 2.2.3 auf Seite 19 dargestellten Datensets. Diese umfassen 1.338 Stichprobenpunkte im Harz und 1.866 im Solling. Der Gesamtdatenpool von 3.204 Einzelstichproben ergibt sich hierbei durch die in allen drei Inventurjahren wiederholte Aufnahme auf insgesamt 1.068 berücksichtigten Stichprobenstandorten. Eine weitere Voraussetzung für die Berücksichtigung der Datensätze für diese Untersuchung ist das Vorhandensein mindestens einer aufgenommenen Fichte im Stichprobenpunkt.

Die Untersuchung erfolgt in einzelnen Schritten auf verschiedenen Referenzebenen. Angefangen mit einem Gesamtmodell über beide Gebiete und alle Inventurjahre hinweg wird die Analyse anschließend hiernach differenziert durchgeführt. Die Untersuchungsergebnisse werden in Form eines Effektwertes, dem Estimate-Wert, ausgegeben und hinsichtlich ihres Signifikanzniveaus im Modell bewertet. Zur Verdeutlichung und einfacheren verbalen Beschreibung der Ergebnisse werden die Effektwerte aller Modelle mit dem Faktor 10 multipliziert in den Tabellen dargestellt. Da von diesem Wert ohnehin keine Aussage über die konkrete Effektstärke abgeleitet werden soll (Schälrisiko nimmt um das x-fache zu oder ab), wirkt sich diese Verschiebung der Kommastellen auch nicht auf die Qualität der Ergebnisse aus. Bewertet wird lediglich die relative Stärke der einzelnen Estimate-Werte innerhalb des Modells. Deren Relation zueinander ändert sich hierdurch nicht.

Das Signifikanzniveau wird durch Sterne wiedergegeben. Hierbei stellen drei Sterne $(* * *)$ ein hochsignifikantes Ergebnis dar $(p<=0,001)$, zwei Sterne stehen für eine Irrtumswahrscheinlichkeit von $0,01 \%$ und ein Stern für eine solche von 0,05\%. Einflussfaktoren, welche zwar noch als erklärende Variable durch das Modell ausgewählt 
und in der Tabelle aufgeführt werden, jedoch nur durch einen Punkt $(\mathrm{p}=0,1)$ oder gar nicht gekennzeichnet sind, erreichen das erforderliche Signifikanzniveau nicht mehr.

\subsubsection{Gesamtmodell}

Bei gemeinsamer Betrachtung beider Untersuchungsgebiete (Tab. 7.1) weist das Gesamtmodell einen hochsignifikanten Unterschied zwischen den Gebieten aus. Auch zwischen den Jahren, welche ebenfalls als erklärende Variable eingegangen sind, wird differenziert - wenn auch mit deutlich geringerer Effektstärke (Estimate $=-0,5)$. Der in dieser Untersuchung neu hinzugekommene Parameter des Fichtenanteils im Stichprobenpunkt (Plot) wird im Modell als negativ mit dem Schälrisiko assoziiert benannt. Demnach steigt das Schälrisiko an den vorhandenen Fichten signifikant mit sinkendem Fichtenanteil im Punkt. Verglichen mit den anderen Modellparametern ist die Effektstärke dieser Variable hoch. Die generelle Baumzahl im Stichprobenpunkt wird hingegen nicht als Indikator verwendet.

Für die Höhe über Normalnull wird im Modell kein statistischer Zusammenhang mit der Schälwahrscheinlichkeit erkannt. Bei der Hangneigung hingegen ist das Signifikanzniveau zwar hoch, der Effektwert mit 0,1 jedoch nur schwach positiv ausgeprägt. Die Hangrichtung wurde in acht Klassen unterteilt. Durch das Modell wurde dann die Ausprägung „Nord“ willkürlich als Referenz für alle weiteren Klassen gewählt und diese entsprechend in Bezug hierauf bewertet. Im Ergebnis werden für sämtliche Expositionsklassen geringere Schälrisikowerte ausgewiesen als für die nördliche Hangrichtung. Bis auf die Nordostklasse erreichen alle Klassen ein ausreichendes bis hohes Signifikanzniveau. Die stärksten Effekte werden in westlicher Ausrichtung dokumentiert (Südwest bis Nordwest: $-5,4$ bis -5,7) sowie für südöstlich orientierte Hänge $(-6,5)$. Der potentielle Strahlungsinput am Stichprobenpunkt erreicht als Variable einen schwach positiven Effektwert. Bei einer angenommenen höheren Sonneneinstrahlung am Punkt steigt demnach auch das Schälrisiko leicht an. Die Exponiertheit im Gelände wird im Modell nicht als erklärende Variable berücksichtigt.

Ebenfalls durch einen positiven Effektwert charakterisiert zeichnet sich der verwendete Rotwildindex über beide Gebiete hinweg als hochsignifikante erklärende Variable ab. Im Vergleich der Effektwerte innerhalb des Modells liegt der Estimate von 2,5 im mittleren Bereich. Für die Baumartenzahl innerhalb der Unterfläche und der Abteilung ergeben sich keine statistischen Zusammenhänge durch die Modellierung. Eine 
Tabelle 7.1: Harz und Solling 2002-06 GLM

\begin{tabular}{lcc}
\hline & \multicolumn{2}{c}{ Harz und Solling 2002-06 } \\
Parameter & Estimate & Sig \\
\hline Gebiet (Solling) & $-3,96$ & $* * *$ \\
Jahr & $-0,53$ & $* * *$ \\
Fichtenanteil im Plot & $-4,66$ & $* *$ \\
Höhe üNN & $-0,01$ & \\
Hangneigung & 0,10 & $* * *$ \\
Hangrichtung (N) & & \\
NO & $-2,04$ & $*$ \\
O & $-2,66$ & $*$ \\
SO & $-6,54$ & $* * *$ \\
S & $-2,41$ & $*$ \\
SW & $-5,41$ & $* * *$ \\
W & $-5,52$ & $* * *$ \\
NW & $-5,67$ & $* * *$ \\
Januarsonne & 0,49 & $*$ \\
Rotwildindex & 2,47 & $* * *$ \\
\hline
\end{tabular}

Die Parameter „Baumzahl im Plot“, „Exponiertheit im Gelände“, „Baumartenzahl in der Abteilung“ und „Baumartenzahl in der Unterfläche" wurden vom Modell nicht als erklärende Variable herangezogen.

Übersicht zu dem mittels Step-AIC erreichten Deltawert des Modells findet sich in Tabelle 7.3 auf Seite 98 .

\subsubsection{Gebietsweise Analyse}

Wie unter 7.1.1 festgestellt, wird das Gebiet als erklärende Variable im Modell ausgewiesen. In der folgenden Untersuchung wird deshalb zwischen Harz und Solling unterschieden und für jedes Gebiet ein gesondertes Modell aufgestellt. Für den Harz gehen die Werte der Inventurjahre 2002, 2004 und 2006 in die Analyse ein, für den Solling jene der Jahre 2002, 2003 und 2006. Die Ergebnisse sind nebeneinander in Tabelle 7.2 dargestellt. Die nun folgende Betrachtung wird jeweils gebietsvergleichend vorgenommen. Die Unterscheidung zwischen den Inventurjahren wird lediglich im Solling (rechte Tabellenhälfte) als signifikanter Einflussfaktor ausgewiesen. Im Harz (links) spielt die- 
Tabelle 7.2: Harz und Solling GLM

\begin{tabular}{lcccc}
\hline & \multicolumn{2}{c}{ Harz $2002-04-06$} & \multicolumn{2}{c}{ Solling 2002-03-06 } \\
Parameter & Estimate & Sig & Estimate & Sig \\
\hline Jahr & 0,34 & & $-2,02$ & $* * *$ \\
Fichtenanteil im Plot & & & $-10,21$ & $* * *$ \\
Hangneigung & 0,12 & $* * *$ & $-0,12$ & $\cdot$ \\
Hangrichtung (Nord) & & & & \\
$\quad$ NO & $-4,47$ & $* *$ & & \\
O & $-3,84$ & $*$ & & \\
SO & $-9,45$ & $* * *$ & & \\
S & $-2,64$ & $*$ & & \\
SW & $-6,54$ & $* * *$ & & \\
W & $-6,70$ & $* * *$ & & \\
$\quad$ NW & $-7,54$ & $* * *$ & & \\
Januarsonne & 0,72 & $* *$ & & \\
Exponiertheit & $-0,25$ & $* * *$ & $-0,48$ & \\
Baumartenzahl Abt & 0,60 & $* * *$ & $-0,75$ & \\
Baumartenzahl Ufl & & & $-1,72$ & \\
Rotwildindex & 2,46 & $* * *$ & & \\
\hline
\end{tabular}

Die Parameter „Baumzahl im Plot“ und „Höhe üNN“ wurden von keinem der Modelle als erklärende Variable herangezogen, die Erholungszonierung im Modell Harz ebenfalls nicht. Alle Werte mit 10 multipliziert.

se Differenzierung innerhalb des Modells keine Rolle für die Prognose des Schälrisikos. Auch der Fichtenanteil im Stichprobenpunkt wirkt sich innerhalb des im Harz untersuchten Datenpools nicht auf das Ergebnis aus. Im Gegensatz hierzu erreicht dieser Parameter im Solling jedoch die höchste Effektstärke innerhalb des Modells und wird als hochsignifikanter Einflussfaktor erkannt. Mit sinkendem Fichtenanteil steigt die Schälwahrscheinlichkeit an den vorhandenen Fichten demnach deutlich an. Wieder wird die generelle Baumzahl im Stichprobenpunkt nicht zur Erklärung der Modelle herangezogen.

Die Geländeparameter werden nur im Harz als erklärende Variablen für das Modell herangezogen. Lediglich bei der Hangneigung zeigt sich auch im Solling ein gering negativer, jedoch nicht signifikanter Effekt. Im Harz ist die Effektstärke ebenfalls gering, wenngleich hochsignifikant und positiv ausgeprägt. Deutlich höhere Effektstärken werden im Harz hingegen für die Hangrichtung ausgewiesen. Dort zeigen sich vergleich- 
bare Ausprägungen zum Gesamtmodell, in dem ja beide Gebiete gemeinsam analysiert wurden (vgl. Tab. 7.1). Dessen Werte waren also offenbar ausschließlich den Zusammenhängen im Harz geschuldet. Gleiches mag für das leicht erhöhte Schälrisiko strahlungsreicher Standorte gelten. Auch hier zeichnet sich lediglich im Harz ein schwach positiver Effekt ab. Die Exponiertheit im Gelände, welche durch das Gesamtmodell nicht als erklärende Variable vorgeschlagen wurde, weist im Einzelmodell Harz nun einen schwach negativ assoziierten Zusammenhang mit dem Schälrisiko auf. Mit steigendem Exponiertheitsgrad sinkt demnach die Wahrscheinlichkeit von Neuschälschäden am Stichprobenpunkt.

Die Baumartenzahl innerhalb der Abteilung wird in beiden Gebieten im Einzelmodell als Variable ausgewiesen. Während im Harz ein positiver Zusammenhang dargestellt wird, zeigt sich im Solling eine negative Verknüpfung. In beiden Fällen ist der Estimate-Wert jedoch gering ausgeprägt. Auf Ebene der Unterfläche entfällt dann im Harz ein statistischer Zusammenhang, während sich im Solling wieder ein Wert analog zur Abteilungsebene zeigt.

Deutliche Unterschiede ergeben sich bei der Betrachtung des Rotwildindexes. Im Harz zeigt sich ein hochsignifikanter mittlerer Effektwert von 2,5. Im Solling erreicht der etwas schwächer, vor allem aber negativ ausgeprägte Wert nicht das erforderliche Signifikanzniveau. Der im Harz zusätzlich untersuchte potentielle Einfluss unterschiedlich stark frequentierter Wanderwege (Erholungszone) wird durch das Modell nicht als erklärende Variable bestätigt. Die durch die beiden Modelle ausgewählten Variablen sind einander auch in Tabelle 7.3 gegenübergestellt. Die Klammern kennzeichnen hierbei berücksichtigte jedoch nicht signifikante Parameter. Die mittels der Methode stepAIC errechneten Start- und Zielwerte sowie das durch die Modelle erreichte Delta sind dort ebenfalls dargestellt. Auch die Werte des vorangegangenen Gesamtmodells sind hier aufgeführt. Im direkten Vergleich zeigt das Modell Harz mit 4,17 \% den relativ höchsten Deltawert, vor dem Gesamtmodell (3,29 \%) und dem Modell für den Solling $(3,08 \%)$. Der Erklärungsgrad gegenüber dem Zufall ist also bei allen drei Modellen gering, doch bieten die Parameter im Harz das im Vergleich höchste Potential.

\subsubsection{Analyse der Inventurjahre je Gebiet}

Unter 7.1.2 wurde gezeigt, dass zumindest im Solling eine Unterscheidung der einzelnen Inventurjahre zur Erklärung des Modells beitragen kann. Da die Trennung der Inventurjahre die Kontinuität als Grad der wiederholten Vorschädigung als zusätzlichen 
Tabelle 7.3: Modellvariablen nach der Methode stepAIC für erste U Harz und Solling

\begin{tabular}{|c|c|c|c|c|}
\hline Datenset & Variablen & AIC start & AIC final & $\Delta \mathrm{AIC}$ \\
\hline Gesamt & $\begin{array}{l}\text { geb }+ \text { jahr }+ \text { fiant }+ \text { slope }+ \text { asp }+ \text { jan } \\
+ \text { index }(\text { hoe })\end{array}$ & 7002,9 & 6772,3 & $3,29 \%$ \\
\hline $\begin{array}{l}\text { Harz } \\
2002-04-06\end{array}$ & $\begin{array}{l}\text { slope }+ \text { asp }+ \text { jan }+ \text { exp }+ \text { abt }+ \text { index } \\
(\text { jahr })\end{array}$ & 3571,3 & 3422,3 & $4,17 \%$ \\
\hline $\begin{array}{l}\text { Solling } \\
\text { 2002-03-06 }\end{array}$ & $\begin{array}{l}\text { jahr }+ \text { fiant }+ \text { abt }+ \text { ufl } \\
\text { (slope }+ \text { index })\end{array}$ & 3326,9 & 3224,4 & $3,08 \%$ \\
\hline
\end{tabular}

Nur die signifikanten ( $\mathrm{p} ; 0,05)$ Modellparameter werden aufgeführt (untere Reihe in Klammern = n.s.)! Getestete Variablen: Hangneigung (slope), Hangrichtung (asp), Exponiertheit (exp), potentielle Einstrahlung (jan), Höhe ü.NN (hoe)

Parameter im letzten Aufnahmejahr zulässt, wird im Folgenden für beide Untersuchungsgebiete hiernach differenziert. In Tabelle 7.4 sind die Ergebnisse der drei gerechneten Modelle für den Harz dargestellt. Da sich viele der untersuchten Parameter in ihrem Effekt nicht von den vorangegangenen Modellen unterscheiden und die konkrete Höhe des Effektwertes in ihrer Aussage nicht überbewertet werden soll, konzentriert sich die folgende Beschreibung auf die Unterschiede und greift die sich wiederholenden Ergebnisse lediglich kurz auf.

So zeigt sich die Baumzahl im Plot erstmalig beim Datenpool des Jahres 2006 als signifikanter Einflussparameter im Modell. Die Effektstärke ist dabei schwach negativ verknüpft. Der hierin enthaltene Fichtenanteil ist wiederum nicht als Variable im Modell verblieben. Der nun einsetzbare Wert der Kontinuität, also die Frequenz der Vorschädigung durch Schäle, steht gemäß seiner Berechnung (vgl. 2.3.1 auf Seite 20) ausschließlich für das Inventurjahr 2006 Verfügung. Für keine der hierzu gebildeten Klassen kann jedoch ein signifikanter Effekt durch das Modell festgestellt werden, gleichwohl die ausgewiesenen Effektwerte erwartungsgemäß mit dem Schälrisiko anzusteigen scheinen.

Bei der Betrachtung der Hangrichtungswerte ergeben sich teilweise gegenteilige Aussagen zwischen den Inventurjahren. So zeigen sich im Jahr 2002 negativ assoziierte Effektwerte für nordöstlich und südwestlich orientierte Hänge, wohingegen im Inventurjahr 2006 für beide positiv gerichtete Werte im Vergleich zur Nordklasse ausgewiesen werden. Die übrigen geländespezifischen Parameter wie Höhe üNN, Hangneigung, 
Tabelle 7.4: Harz GLM

\begin{tabular}{|c|c|c|c|c|c|c|}
\hline \multirow[b]{2}{*}{ Parameter } & \multicolumn{2}{|c|}{ Inventurjahr 2002} & \multicolumn{2}{|c|}{ Inventurjahr 2004} & \multicolumn{2}{|c|}{ Inventurjahr 2006} \\
\hline & Estimate & Sig & Estimate & Sig & Estimate & Sig \\
\hline Baumzahl im Plot & $-0,31$ & & 0,42 & . & $-0,88$ & $* * *$ \\
\hline \multicolumn{7}{|l|}{ Fichtenanteil im Plot } \\
\hline Kontinuität (nie) & - & & - & & & \\
\hline ehemals & & & & & 1,03 & \\
\hline sporadisch & & & & & 1,85 & \\
\hline häufig & & & & & 1,91 & \\
\hline stets & & & & & 1,98 & \\
\hline Höhe üNN & $-0,01$ & . & & & & \\
\hline Hangneigung & 0,13 & $* *$ & 0,17 & $* * *$ & & \\
\hline \multicolumn{7}{|l|}{ Hangrichtung (Nord) } \\
\hline NO & $-11,83$ & $* * *$ & $-1,98$ & & 6,18 & $*$ \\
\hline $\mathrm{O}$ & $-4,72$ & & $-7,80$ & $* *$ & 4,14 & \\
\hline $\mathrm{SO}$ & $-7,13$ & $* *$ & $-12,24$ & $* * *$ & $-2,41$ & \\
\hline S & $-5,88$ & $*$ & $-2,69$ & & 3,73 & . \\
\hline SW & $-10,65$ & $* * *$ & $-8,18$ & $* * *$ & 5,72 & $*$ \\
\hline $\mathrm{W}$ & $-9,05$ & $* * *$ & $-8,32$ & $* * *$ & 3,06 & \\
\hline NW & $-5,09$ & $*$ & $-11,10$ & $* * *$ & $-0,51$ & \\
\hline $\mathrm{P}$ & & & $-117,13$ & & 9,79 & \\
\hline Januarsonne & 1,17 & $*$ & & & & \\
\hline Exponiertheit & $-0,24$ & $*$ & & & $-0,28$ & $* * *$ \\
\hline Baumartenzahl Abt & & & 0,74 & $* *$ & 0,78 & $* * *$ \\
\hline \multicolumn{7}{|l|}{ Baumartenzahl Ufl } \\
\hline Rotwildindex & 1,94 & & 2,51 & $*$ & & \\
\hline \multicolumn{7}{|l|}{ Erholungszone (kaum) } \\
\hline mittel & $-6,36$ & $*$ & & & & \\
\hline stark & 1,82 & & & & & \\
\hline
\end{tabular}

Januarsonne und Exponiertheit weisen im Vergleich zur gemeinsamen Analyse keine weiteren Besonderheiten auf. Dies gilt auch für die Information über Baumartenzahlen der Umgebung. Beim Rotwildindex fällt jedoch auf, dass lediglich im Jahr 2004 ein signifikanter Einfluss durch das Modell attestiert wird. Die im Gesamtmodell des Harzes nicht als Variable herangezogene Erholungszonierung zeigt bei getrennter Betrachtung der Jahre nun einen deutlich negativen Effekt bei der mäßig frequentierten 
Tabelle 7.5: Solling GLM

\begin{tabular}{|c|c|c|c|c|c|c|}
\hline \multirow[b]{2}{*}{ Parameter } & \multicolumn{2}{|c|}{ Inventurjahr 2002} & \multicolumn{2}{|c|}{ Inventurjahr 2003} & \multicolumn{2}{|c|}{ Inventurjahr 2006} \\
\hline & Estimate & Sig & Estimate & Sig & Estimate & Sig \\
\hline \multicolumn{7}{|l|}{ Baumzahl im Plot } \\
\hline Fichtenanteil im Plot & $-7,53$ & $* *$ & $-11,15$ & $* * *$ & $-17,32$ & $* * *$ \\
\hline \multicolumn{7}{|l|}{ Kontinuität (nie) } \\
\hline ehemals & & & & & 1,44 & \\
\hline sporadisch & & & & & 188,15 & \\
\hline häufig & & & & & 193,20 & \\
\hline stets & & & & & 203,96 & \\
\hline \multicolumn{7}{|l|}{ Höhe üNN } \\
\hline Hangneigung & $-0,25$ & $*$ & 0,30 & $* *$ & & \\
\hline \multicolumn{7}{|l|}{ Hangrichtung (Nord) } \\
\hline $\mathrm{NO}$ & & & $-2,86$ & & & \\
\hline $\mathrm{O}$ & & & $-0,42$ & & & \\
\hline $\mathrm{SO}$ & & & $-2,13$ & & & \\
\hline $\mathrm{S}$ & & & $-7,23$ & $*$ & & \\
\hline SW & & & $-9,80$ & $* *$ & & \\
\hline $\mathrm{W}$ & & & $-11,95$ & $* * *$ & & \\
\hline NW & & & $-8,02$ & $* *$ & & \\
\hline $\mathrm{P}$ & & & - & & & \\
\hline Januarsonne & & & & & $-2,91$ & . \\
\hline Exponiertheit & & & & & 0,41 & $* * *$ \\
\hline Baumartenzahl Abt & $-0,33$ & & $-1,20$ & $* * *$ & & \\
\hline Baumartenzahl Ufl & $-0,55$ & & & & & \\
\hline Rotwildindex & & & & & $-20,49$ & $* * *$ \\
\hline
\end{tabular}

im Vergleich zur kaum genutzten Zone. Für vermeintlich stark durch den Menschen frequentierte Bereiche wird allerdings kein signifikant höherer Wert ausgegeben.

Durch die Trennung nach Inventurjahren ergibt sich für den Solling, ebenso wie zuvor für den Harz, ein differenziertes Bild der einzelnen Parameter. In Tabelle 7.5 sind die Ergebnisse der drei Modellrechnungen aufgeführt. Im Vergleich der Inventurjahre untereinander wird deutlich, dass kaum ein Parameter durchgängig als signifikante erklärende Variable in den Modellen verwendet wird. Konstant in allen drei Modellen verbleibt nur der Parameter des Fichtenanteils im Plot. Wie schon im Gesamtmodell für den Solling ausgewiesen, steht dieser Wert in einer negativen Beziehung zum Schälrisiko. Geringere Fichtenanteile im Stichprobenpunkt erhöhen also die Wahr- 
scheinlichkeit, Schälschäden an den vorhandenen Fichten vorzufinden. Ebenfalls analog zum Gesamtmodell der drei Jahrgänge wird die Gesamtbaumzahl im Plot wiederum nicht als erklärende Variable hinzugezogen. Auch im Solling kann der Grad der vorangegangenen Schälbelastung ausschließlich für das letzte Inventurjahr in seiner Eigenschaft als Einflussfaktor untersucht werden (vgl. 2.3.1 auf Seite 20). Wie im Harz zeigen sich auch hier hohe Effektwerte in ansteigender Reihe zur Vorschädigung, doch bleibt dieser angenommene Zusammenhang erneut unterhalb der Signifikanzgrenze.

Bei den Geländeparametern zeigen sich über die Jahre uneinheitliche, zumeist aber schwache Werte. Die Höhe über Normalnull bietet offenbar in keinem Inventurjahr Erklärungspotential. Während für die Hangneigung im Jahr 2002 ein negativer Zusammenhang zum Schälrisiko angenommen wird, kehrt sich dieser Effekt im Modell der Folgeinventur wieder um. Die Hangrichtung wird lediglich im Jahr 2003 als Einflussfaktor erkannt, wobei lediglich die westlich orientierten Standorte signifikant niedrigere Schälwahrscheinlichkeiten erwarten lassen, als solche nördlicher Ausrichtung. Im Gesamtmodell über alle drei Jahre war dieser Parameter nicht im Modell verblieben. Der potentielle Strahlungsinput erreicht keine signifikanten Werte und auch die Exponiertheit im Gelände wird lediglich im Jahr 2006 als Variable durch das Modell verwendet.

Während die Baumartenzahl über alle Inventuren hinweg sowohl auf Abteilungs- als auch auf Unterflächenebene signifikante Effektwerte aufgewiesen hatte, zeigt sich bei der Einzelbetrachtung nur auf Basis der Abteilung ein signifikanter Negativeffekt im Jahr 2003. Der Rotwildindex kommt ebenfalls nur in einem Inventurjahr als erklärender Faktor zum Tragen. Im Modell des Jahres 2006 wird hierfür ein positiv assoziierter Effekt unterstellt, der als hochsignifikant ausgegeben wird.

In der Art, wie es zuvor für die beiden Untersuchungen über alle Inventurjahre hinweg aufgezeigt wurde (vgl. Tab. 7.3 auf S. 98), sollen auch die nun nach Inventurjahren getrennten Modelle in ihrem Modelleffekt gegenübergestellt werden. In Tabelle 7.6 sind die ausgewählten Variablen der jeweiligen Modelle deshalb noch einmal aufgelistet. Die in Klammern gesetzten Parameter kennzeichnen im Modell berücksichtigte, jedoch nicht signifikante Parameter. Zum Vergleich der Modelle untereinander ist als Anhaltswert erneut das relative Delta nach der stepAIC-Methode errechnet und, neben den Start- und Zielwerten, in der Tabelle angegeben worden.

Ohne diesen Werten eine zu hohe Aussagekraft zuzusprechen, lassen sich doch erhebliche Unterschiede zwischen den Inventurjahren feststellen. So weichen die Zielwerte im Jahr 2002 sowohl im Harz als auch im Solling kaum von ihren Startwerten ab. Durch 
Tabelle 7.6: Modellvariablen nach der Methode stepAIC für Harz und Solling

\begin{tabular}{|c|c|c|c|c|}
\hline Datenset & Variablen & AIC start & AIC final & $\Delta \mathrm{AIC}$ \\
\hline Harz 2002 & $\begin{array}{l}\text { slope }+ \text { asp }+ \text { jan }+ \text { exp }+ \text { ez } \\
(\text { plot }+ \text { hoe }+ \text { index })\end{array}$ & 1092,5 & 1064,9 & $2,53 \%$ \\
\hline Harz 2004 & $\begin{array}{l}\text { slope }+ \text { asp }+ \text { abt }+ \text { index } \\
\text { (plot) }\end{array}$ & 1184,3 & 1119,6 & $5,46 \%$ \\
\hline Harz 2006 & $\begin{array}{l}\text { plot }+ \text { asp }+\exp +\text { abt } \\
\text { (kont) }\end{array}$ & 1293,2 & 857,9 & $33,66 \%$ \\
\hline Solling 2002 & $\begin{array}{l}\text { fiant }+ \text { slope } \\
(\text { abt }+ \text { ufl })\end{array}$ & 1413,2 & 1401,9 & $0,80 \%$ \\
\hline Solling 2003 & $\begin{array}{l}\text { fiant }+ \text { slope }+ \text { asp }+ \text { abt } \\
(-)\end{array}$ & 1028,2 & 974,1 & $5,26 \%$ \\
\hline Solling 2006 & $\begin{array}{l}\text { fiant }+ \text { exp }+ \text { index } \\
(\text { kont }+ \text { jan })\end{array}$ & 816,9 & 534,4 & $34,58 \%$ \\
\hline
\end{tabular}

$\mathrm{P}=$ Plateau; Nur die signifikanten ( $\mathrm{p}$; 0,05) Modellparameter werden aufgeführt (untere Reihe in Klammern = n.s.)! Getestete Variablen: Hangneigung (slope), Hangrichtung (asp), Exponiertheit (exp), potentielle Einstrahlung (jan), Höhe ü.NN (hoe)

die Aufnahme der erklärenden Variablen in die Modelle ändert sich der AIC-Wert im Harz demnach nur geringfügig um 2,5 \% und im Solling mit einer relativen Differenz von 0,8 \% fast gar nicht. Mit etwas über $5 \%$ Veränderung liegt das Effektpotential in der Folgeinventur 2003 im Solling und 2004 im Harz zwar deutlich darüber, erreicht insgesamt aber ebenfalls nur geringe Werte.

Im Jahr 2006 reduziert sich der AIC-Startwert der Modelle durch Hinzunahme der Variablen dann aber um ein Drittel (um $34 \%$ im Harz und um $35 \%$ im Solling). Diese Werte liegen nicht nur weit über denen der anderen Inventurjahre, sondern setzen sich in gleicher Weise auch von denen der Gesamtuntersuchung (Tab. 7.3) ab. Als exklusiver Parameter kommt im Jahr 2006 die berechnete Kontinuität der Vorschädigung im Stichprobenpunkt hinzu. Auch wenn dieser Parameter nicht als signifikante Einflussvariable ausgewiesen wird, wird er doch in beiden Gebieten als Faktor im Modell belassen und ist dort sehr wahrscheinlich für die hohen Deltawerte verantwortlich. Bereits in den Modellergebnissen selbst (für den Harz in Tab. 7.4 und für den Solling in Tab. 7.5 dargestellt) werden für die Klassen der Kontinuität sehr hohe Effektwerte deutlich. 


\subsubsection{Modellierung nach Parametergruppen}

Abschließend sollen die zur Verfügung stehenden Parameter noch einmal über alle Inventurjahre hinweg, nur getrennt nach den Untersuchungsgebieten, getestet werden. Der Fokus liegt jetzt aber in der gesonderten Betrachtung der reliefabhängigen Paramter, die in einer Gruppe zusammengefasst sind sowie den übrigen Parametern, welche ebenfalls für sich genommen in einem Modell untersucht werden sollen. Hinter der Trennung dieser Parametergruppen steht die Frage, welche Aussagen zum Schälrisiko sich möglicherweise allein durch die Kenntnis der geografischen Bedingungen der Gebiete treffen lassen.

In Tabelle 7.7 sind die Ergebnisse des Geodatenmodells für beide Gebiete getrennt aufgeführt. Im Vergleich der beiden Gebiete wird erneut deutlich, dass die Geländestruktur im Harz offenbar größere Auswirkungen auf das Modell hat, als dies im Solling der Fall ist. Bis auf die Höhe üNN werden im Harz sämtliche Parameter als signifikante Einflussfaktoren verwendet. Im Solling verbleiben hingegen nur einige Expositionsklassen als signifikante erklärende Variablen im Modell. Die potentielle Strahlungsmenge am Stichprobenpunkt wird zwar als Einflussgröße ausgewiesen, erreicht aber nicht das definierte Signifikanzniveau. Analog zum Wert im Harz wird auch hier ein positiver Zusammenhang zum Schälrisiko angenommen.

Anders verhält es sich mit dem Grad der Exponiertheit im Gelände. Herausgestellten Geländepunkten im Harz wird durch eine negative Beziehung (Vorzeichen des EstimateWertes) eine reduzierte Schälanfälligkeit unterstellt. Stichprobenpunkte, welche sich in geschützterer Lage im Raum befinden, weisen damit ein erhöhtes Schälrisiko auf. Gleiches gilt im Harz für Standorte in steilen Lagen. Mit zunehmender Hangneigung nimmt demnach auch die Schälwahrscheinlichkeit an den Stichprobenpunkten zu. Die Faktoren Hangneigung und Grad der Exponiertheit im Raum sind im Harz schwach negativ korreliert ( $\mathrm{r}=-0,55$ nach Pearson), was ebenfalls auf die Beziehung der Hangneigung zum Strahlungspotential zutrifft $(\mathrm{r}=-0,47)$. Die Exponiertheit und das Strahlungspotential am Stichprobenpunkt sind hingegen schwach positiv korreliert $(\mathrm{r}=0,58)$.

Die Ergebnisse aller anderen untersuchten Parameter sind in Tabelle 7.8 dargestellt. Wie bereits in den vorangegangenen Modellen festgestellt, nimmt die Unterscheidung zwischen den Jahren nur im Solling einen signifikanten Einfluss auf die Risikobewertung. Die Baumzahl im Stichprobenpunkt zeigt sich jahresübergreifend in keinem der Gebiete als Einflussfaktor. Mit abnehmendem Fichtenanteil im Plot steigt zumindest im 
7. Generalisierte Lineare Modelle

Tabelle 7.7: GLM Geodaten Harz und Solling

\begin{tabular}{lrlcc}
\hline & \multicolumn{2}{c}{ Harz } & \multicolumn{2}{c}{ Solling } \\
Parameter & Estimate & Sig & Estimate & Sig \\
\hline Höhe üNN & $-0,01$ & $\cdot$ & & \\
Hangneigung & 0,14 & $* * *$ & & \\
Hangrichtung (Nord) & & & & \\
$\quad$ NO & $-4,54$ & $* *$ & 0,14 & \\
O & $-3,69$ & $*$ & $-2,01$ & $*$ \\
SO & $-9,16$ & $* * *$ & $-4,11$ & $* *$ \\
S & $-2,35$ & $\cdot$ & $-4,53$ & $* * *$ \\
SW & $-6,57$ & $* * *$ & $-3,92$ & $* *$ \\
W & $-6,24$ & $* * *$ & $-4,67$ & $*$ \\
NW & $-7,43$ & $* * *$ & $-4,69$ & \\
$\quad$ P & $-1,17$ & & $-8,17$ & \\
Januarsonne & 0,72 & $* *$ & 1,08 & \\
Exponiertheit & $-0,22$ & $* *$ & & $*$ \\
\hline
\end{tabular}

Solling die Schälwahrscheinlichkeit an den restlichen Fichten deutlich an. Beim Faktor der Baumartenvielfalt ergeben sich erneut teilweise gegensprüchliche Ergebnisse. Auf der Ebene der Forstabteilung untersucht, wird in beiden Modellen ein signifikanter Effekt für diesen Faktor ausgewiesen. Im Harz wird allerdings ein positiver Zusammenhang unterstellt, während im Solling ein Anstieg des Schälrisikos bei Abnahme der

Tabelle 7.8: GLM Rest Harz und Solling

\begin{tabular}{lcccc}
\hline & \multicolumn{2}{c}{ Harz } & \multicolumn{2}{c}{ Solling } \\
Parameter & Estimate & Sig & Estimate & Sig \\
\hline Jahr & 0,31 & & $-2,0$ & $* * *$ \\
Baumzahl im Plot & & & $-9,74$ & $* * *$ \\
Fichtenanteil im Plot & & $* * *$ & $-0,53$ & $* *$ \\
Baumartenzahl Abt & 0,58 & & $-0,73$ & $* *$ \\
Baumartenzahl Ufl & & $* * *$ & $-1,46$ & \\
Rotwildindex & 3,16 & & & \\
Erholungszone (kaum) & & & & \\
$\quad$ mittel & & & & \\
$\quad$ stark & & & &
\end{tabular}


7.1. Ergebnisse

Tabelle 7.9: Modellvariablen nach der Methode stepAIC für Gruppen Harz und Solling

\begin{tabular}{|c|c|c|c|c|}
\hline Datenset & Variablen & AIC start & AIC final & $\Delta \mathrm{AIC}$ \\
\hline Harz Geodaten & $\begin{array}{l}\text { slope }+ \text { asp }+ \text { jan }+ \text { exp } \\
\text { (hoe) }\end{array}$ & 3571,3 & 3456,1 & $3,23 \%$ \\
\hline Harz Sonstige & $\begin{array}{l}\text { abt }+ \text { index } \\
(\text { jahr })\end{array}$ & 3571,3 & 3525,8 & $1,27 \%$ \\
\hline Solling Geodaten & $\begin{array}{l}\text { asp } \\
(\text { jan })\end{array}$ & 3326,9 & 3322,3 & $0,14 \%$ \\
\hline Solling Sonstige & $\begin{array}{l}\text { jahr }+ \text { fiant }+ \text { abt }+ \text { ufl } \\
\text { (index) }\end{array}$ & 3326,9 & 3226,0 & $3,03 \%$ \\
\hline
\end{tabular}

Nur die signifikanten (p ; 0,05) Modellparameter werden aufgeführt (untere Reihe in Klammern $=$ n.s.)! Getestete Variablen: Hangneigung (slope), Hangrichtung (asp), Exponiertheit (exp), potentielle Einstrahlung (jan), Höhe ü.NN (hoe)

Baumartenzahl in der Abteilung angenommen wird. Auf Ebene der Unterfläche, also in lokal engerem Zusammenhang mit dem Stichprobenpunkt stehend, zeigt sich lediglich im Solling ein wiederum negativ wirkender Effekt. Ein signifikantes und in seinem Effektwert relativ starkes Ergebnis erfährt der Rotwildindex im Modell für den Harz. Beschränkt auf die in diesem Modell untersuchten Parameter wirkt sich die Höhe der lokalen Rotwildstrecke hochsignifikant und am stärksten von allen Faktoren auf die zu erwartenden Schälschäden aus. Es wird ein positiver Zusammenhang ausgewiesen. Der Rotwildindex und die Schälschäden steigen also gemeinsam an. Im Solling kann dieser Zusammenhang durch das Modell nicht nachgewiesen werden. Auch die Erholungszonierung im Harz führt nicht zu einer erklärenden Variablen im Modell.

Betrachtet man die Gesamteffekte der Modelle nach der Methode stepAIC nun wieder nebeneinander (Tab. 7.9), so zeigt sich zunächst deren bereits zu erwartende allgemein schwache Ausprägung. Der Vergleich untereinander zeigt aber auch, dass durch die Hereinnahme der Gruppe reliefbedingter Parameter im Harz immerhin ein Effekt im Modell erzielt wird (3,2 \%), während im Solling durch die gleiche Parametergruppe so gut wie keine Veränderung herbeigeführt wird (0,1\%). Gegenteilig findet im Solling jedoch eine merkliche Verringerung des AIC-Wertes durch die Analyse der restlichen Parameter statt. Mit rund $3 \%$ Veränderung erreicht diese Gruppe damit einen vergleichbaren Wert wie die Geländeparameter im Harz. Diese Parametergruppe wirkt sich im Harz wiederum nur sehr schwach im Modell aus (1,3\%). 


\subsection{Zusammenfassung}

Anders als im Solling haben die Modelle im Harz vor allem bei der Untersuchung der reliefbasierten Parameter zu wiederkehrenden Ergebnissen geführt. Sowohl im Modell über alle Inventurjahre hinweg (vgl. Tab. 7.2, S. 96) als auch in der Betrachtung der einzelnen Inventurjahre (vgl. Tab. 7.4. S. 99) zeigen sich für die Geländeparameter konstante Effekte. Zwar variiert deren Stärke, sodass in einzelnen Modellen keine signifikanten Werte erreicht werden, doch bleibt die Ausprägung, also der angenommene Zusammenhang mit dem Schälrisiko, über die gesamte Untersuchungsreihe hinweg gleich. Um bei der Modellierung den Einfluss anderer Faktoren auszublenden, wurde unter 7.1.4 der Blick noch einmal speziell auf die Gruppe der Geländeparameter gerichtet. Auch die dortigen Ergebnisse (vgl. Tab. 7.7, S. 104 weisen auf Zusammenhänge zwischen Geländeausprägung und Schälrisiko hin und bestätigen die zuvor festgestellten Effektrichtungen.

Der Rotwildindex wird über beide Gebiete hinweg als hochsignifikante erklärende Variable ausgewiesen, während die lediglich für das letzte der drei Inventurjahre rückblickend eingesetzte Variable der Kontinuität keinen Effekt zeigt. Auch für die Anzahl der Baumarten ergeben sich im Gesamtmodell keine statistischen Zusammenhänge mit der Schälintensität. Gebietsweise untersucht, zeigen sich im Harz jedoch schwach positive und im Solling schwach negative Korrelationen. Der Fichtenanteil im Stichprobenpunkt weist jedoch einen Anstieg des Schälrisikos an den vorhandenen Fichten aus, wenn der Fichtenanteil im Punkt insgesamt abnimmt. Dieser im Modell stark ausgeprägte Effekt zeigt sich jedoch nur im Solling.

Zwischen der Höhenlage der Stichprobenpunkte im Gelände und der Schälwahrscheinlichkeit wird kein statistischer Zusammenhang im Modell erkannt. Insgesamt aber führt die Untersuchung der reliefbasierten Parameter im Harz zu wiederkehrenden Ergebnissen. So ergibt sich für Hänge ein schwacher Anstieg der Schälrate mit zunehmender Neigung. Nach Norden exponierte Hanglagen weisen im Vergleich zu allen anderen Richtungen zudem die höchsten Schälraten auf, während westlich und südöstlich ausgerichtete Lagen die geringsten Werte zeigen. Mit einem höheren Strahlungsinput am Punkt steigt auch das Schälrisiko leicht an. Eine starke Exponiertheit im Gelände wiederum verringert das Schälrisiko deutlich. Im Solling ergeben sich für die Geländeparameter hingegen keine Effekte. 


\section{Konsquenzen für die Hypothesen}

\section{Hypothese I}

Punkte mit aktueller Schälschädigung sind auch Orte mit hoher Wiederholungsrate in der Schälschädigung.

Bei der Betrachtung der Chancenverhältnisse (Kapitel 5) zeigt sich in beiden Untersuchungsgebieten ein tendenziell höheres Risiko für Neuschäle im Stichprobenpunkt, wenn dort bereits zuvor Bäume geschält worden waren. Insbesondere bei extremen Abweichungen vom Mittel verschiebt sich auch das lokale Chancenverhältnis im Vergleich zum Gesamtdurchschnitt.

Die durchgeführten Regressionsrechnungen (Kaptitel 6) weisen die Information über das Vorkommen von Altschälschäden für den Harz in allen Modellen als erklärende Variable aus. Im Solling wird dieser Zusammenhang hingegen nur in einem der drei Modelle detektiert. Die im GLM lediglich für das letzte der drei Inventurjahre rückblickend eingesetzte Variable der Kontinuität zeigt keinen Effekt.

\section{Hypothese II}

Die Höhe der Rotwildjagdstrecke eines Revieres korreliert mit der Höhe des Schälschädigungsgrades im selben Gebiet.

Das Chancenverhältnis für Neuschäle ändert sich mit Variation der Rotwildstrecke und zeigt ein höheres Risiko bei hohem Jagdstreckenaufkommen. Im Solling wird im Gebiet mit dem höchsten Rotwildabschuss allerdings das geringste Schälrisiko ausgewiesen.

Die Regressionsmodelle weisen die Jagdstreckenhöhe im Solling als den stärksten, negativ korrelierten Faktor aus. Im Harz ergibt sich hingegen kein Zusammenhang. Im GLM wiederum wird der Rotwildindex ausschließlich im Harz als erklärende Variable ausgewiesen. 


\section{Hypothese III}

Stichprobenpunkte in reich strukturierten Beständen mit mehreren Baumarten weisen ein geringeres Schälrisiko auf als solche in gleichaltrigen Reinbeständen.

Bei der Betrachtung des Bestandesalters weisen jüngere Bestände in beiden Gebieten ein deutlich erhöhtes Schälrisiko gegenüber der mittleren Wahrscheinlichkeit auf. Bei der Klassifizierung nach Baumartenzahlen zeigt sich hingegen weder im Harz noch im Solling eine Tendenz der Chancenverhältnisse geschälter zu ungeschälter Einheiten.

In den Regressionsrechnungen wird die Zunahme der Baumartenzahl im Harz tendenziell als Faktor für die Erhöhung des Neuschälrisikos ausgewiesen, während im Solling eine Verringerung des Risikos mit der Zunahme der Baumarten je Fläche einhergeht.

In den Modellierungen mittels GLM ergeben sich für die Baumartenzahl im Gesamtmodell keine statistischen Zusammenhänge mit der Schälintensität. Gebietsweise untersucht, zeigen sich im Harz schwach positive und im Solling schwach negative Korrelationen. Der in dieser Untersuchung neu hinzugekommene Parameter des Fichtenanteils im Stichprobenpunkt weist jedoch einen Anstieg des Schälrisikos an den vorhandenen Fichten aus, wenn der Fichtenanteil im Punkt insgesamt abnimmt. Dieser stark ausgeprägte Effekt zeigt sich jedoch nur im Solling.

\section{Hypothese IV}

An Stichprobenpunkten tieferer Lagen ist die Wahrscheinlichkeit eines Winterneuschälschadens größer als an jenen höherer Lagen. Daneben steigt die Wahrscheinlichkeit in strahlungsintensiven und in windabgewandten Lagen.

Die Höhenlage ergibt in keinem Gebiet eine klare Abweichung des Schälrisikos vom Mittelwert. Die Chancenverhältnisse zeigen jedoch an sehr schwach und sehr stark geneigten Standorten ein verringertes Neuschälrisiko an. Im Solling ergeben sich erhöhte Werte in Nord- bis Ostrichtung der Hänge. Eine geringe Exponiertheit im Gelände führt im Harz zu erhöhten Schälwahrscheinlichkeiten und auch bei sehr geringen Strahlungswerten wird dort ein stark reduziertes Schälrisiko festgestellt.

In den Regressionsmodellen für den Harz werden die Hangrichtung und der potentielle Strahlungsinput als erklärende und signifikante Variablen im Modell erkannt. Im Solling verbleibt kein Geländeparameter im finalen Regressionsmodell. 
Auch im GLM wird kein statistischer Zusammenhang der Höhenlage mit der Schälwahrscheinlichkeit erkannt. Insgesamt aber haben die Modelle im Harz bei den reliefbasierten Parameter zu wiederkehrenden Ergebnissen geführt. Bei der Hangneigung ergibt sich ein schwach positiv ausgeprägter Effektwert. Nach Norden exponierte Hänge weisen die höchsten Schälraten auf, während westlich und südöstlich ausgerichtete Lagen die geringsten Werte zeigen. Bei höherem Strahlungsinput am Punkt steigt auch das Schälrisiko leicht an. Eine starke Exponiertheit im Gelände verringert das Schälrisiko deutlich. Im Solling ergeben sich hierfür keine Effekte. 



\section{Diskussion}

\subsection{Betrachtung der Voruntersuchung}

Wie in der Einleitung ausführlich dargestellt, wurden für diese Arbeit bereits im Vorfeld binär logistische Regressionsrechnungen als Form der Datenanalyse festgelegt. Auch die hierzu vorab veröffentlichten Voruntersuchungen hatten sich auf diese Art der statistischen Auswertung gestützt. Die abhängige Variable wird in einem solchen Fall auf die Ausprägungen 0 und 1, also ungeschält und geschält, beschränkt. Ein Stichprobenpunkt, welcher vollkommen frei von Neuschälschäden ist, erhält demnach den Wert 0 . Schon bei einer einzigen als geschält dokumentierten Fichte wird der Stichprobenpunkt hingegen mit dem Wert 1 klassifiziert. Die absolute und auch relative Schadenshöhe im Punkt erfährt also keine Beachtung.

In dieser Betrachtungsweise lauern jedoch Fallstricke. Diese sind in der Voruntersuchung nicht beachtet worden. Vollständig besetzte Stichprobenpunkte und solche mit weniger als 18 Bäumen gingen gleichwertig in die Voranalyse ein. Es wurde zwar zwischen Punkten mit 18, 12 und 6 erfassten Bäumen unterschieden, doch ging diese Information lediglich als unabhängige Variable in das Modell ein. Stichprobenpunkte mit unvollständiger Baumzahl wurden dahingehend interpretiert, dass der dortige Aufnahmepunkt in Teilen von einem Forst- oder Wanderweg gekreuzt wurde. Je nach Lage seien dann dort ein oder zwei Segmente der Klumpenstichprobe ausgefallen, da auf oder nahe des Weges keine Bäume zur Verfügung gestanden hätten. Die Variable wurde also als Maß der Wegenähe verwendet (KIffner ET AL.). Allein diese Annahme muss als fraglich gewertet werden, da es grundsätzlich verschiedene Ursachen, wie beispielsweise Blößen, für unvollständig besetzte Stichprobenpunkte geben kann.

Das im Sinne der Modellqualität weitreichendere Problem aber liegt in der Missachtung dieser Information für die abhängige Variable. Bei deren Analyse im binär logistischen Regressionsmodell werden sich verändernde Wahrscheinlichkeiten (odds) einander gegenübergestellt (odds ratio), wenn sich die unabhängigen Variablen um ei- 
ne Einheit verschieben. Die Chance des Stichprobenpunktes, als abhängige Variable mit 1 codiert zu werden, ist nun bei unvollständig besetzten Punkten aber viel geringer, denn je mehr Bäume im Punkt auf einen Neuschälschaden hin untersucht werden, desto höher ist ja die Wahrscheinlichkeit, dass einer von ihnen tatsächlich einen solchen Schaden aufweist.

Die Voruntersuchung selbst zeigt genau diese Verschiebung der Wahrscheinlichkeiten auch, denn Stichprobenpunkte mit 12 oder gar nur 6 aufgenommenen Bäumen erreichen häufiger den Wert 0. Durch die vorherige Annahme einer räumlichen Nähe dieser Punkte zu Wegen fällt die Interpretation dieses Ergebnisses dann aber entsprechend anders aus. Der kausale Zusammenhang von geringerer Schälwahrscheinlichkeit entlang von (vermuteten) Wegen wird auf die dort vermeindlich erhöhte Präsenz des Menschen zurückgeführt. Die unterschiedlichen Bedingungen der Stichprobenpunkte selbst dürften jedoch für diese Verschiebung von Wahrscheinlichkeiten verantwortlich sein, um die es im Modell geht. Dieser Effekt wird durch die Beschränkung auf die Fichte noch verschärft. So sind zwar ausschließlich Schälschäden an Fichten für die Codierung der abhängigen Variablen als relevant definiert, deren tatsächlicher Anteil im Stichprobenpunkt wird aber wiederum nicht berücksichtigt.

\subsubsection{Konsequenzen für die eigene Analyse}

Um diesen ungewollten Effekt in der eigenen Arbeit zu vermeiden, wurde der Datenpool vorab in homogene und heterogene Datensets getrennt. In die homogenen Datensets gingen dabei nur jene Stichprobenpunkte ein, welche mit 18 aufgenommenen Bäumen vollständig besetzt waren. Bei diesen Bäumen musste es sich zudem auschließlich um Fichten handeln. Alle anderen Stichprobenpunkte sollten nicht in die binäre logistische Regressionsrechnung eingehen und wurden in dieser Untersuchung als heterogen bezeichnet. Für eine statistisch saubere Analyse wird diese Grenzziehung als notwendig erachtet. Sie bringt jedoch unweigerlich den Nachteil mit sich, dass sich der Stichprobenumfang massiv verringert.

Durch die Vielzahl der in die binären logistischen Regressionsrechnungen eingehenden Variablen und deren Interaktion sind diese Modelle in ihrer Aussage recht labil. Die vorab zusätzlich durchgeführten nicht-parametrischen statistischen Tests sollten deshalb eine Orientierung bieten, um die komplexen Ergebnisse der anschließenden Regressionen sicherer interpretieren und bewerten zu können. 
In der weitergehenden Untersuchung, losgelöst von der vorgegebenen Analyseart, wurden dann auch die heterogenen Stichprobenpunkte berücksichtigt. Hierzu musste eine Analyse gewählt werden, in die die geschälten Fichten als relativer Wert in Bezug auf die Gesamtfichtenzahl im Plot eingehen konnten (GLM). So war es möglich, den gesamten Stichprobenumfang zu nutzen und darüber hinaus den Informationsverlust durch die 0/1-Codierung zu umgehen.

Bei der Betrachtung der Ergebnisse zeigt sich, dass sich die Aussagen zwischen den Analysemodellen aber auch innerhalb der Modelle für dieselben Parameter unterscheiden. So nimmt die Schäle mal mit steigender Höhe üNN sowohl zu als auch ab. Die Beschreibung der Ergebnisse beschränkt sich deshalb auch auf die Richtung und die relative Effektstärke in Bezug auf die anderen Variablen des Modells. Auf eine detaillierte Gegenüberstellung der Effektstärke in Zahlenwerten (wie ändert sich das odds ratio bei Zunahme des Faktors um eine Einheit) wurde bewusst verzichtet. Bei oftmals gegenteiligen inhaltlichen Aussagen der Variablen wurde der mathematisch hergeleiteten Effektstärke deshalb keine quantitative Aussagefähigkeit zugestanden, auch um die Präsentation von Scheingenauigkeiten zu vermeiden.

Aus diesem Grund wird in der vorliegenden Arbeit auch auf die Einbeziehung der Schneehöhe als unabhängige Variable verzichtet, obwohl diese in der Vorveröffentlichung bereits als signifikanter Einflussfaktor herausgestellt wurde. Hierzu hatte man in der Untersuchung sämtlichen Stichprobenpunkten einen Wert der angenommenen, mittleren Schneehöhe im Winterhalbjahr zugewiesen. Als Basis hierfür hatten Messdaten regionaler Wetterstationen gedient, deren täglich protokollierte Schneehöhenwerte zunächst über die Summe aller Tage mit Schneelage arithmetisch gemittelt wurden. Ausgehend von der geografischen Nähe der einzelnen Stichprobenpunkte zu den Messstationen wurden diese gemittelten Schneehöhenwerte dann über alle im Modell vorkommenden Höhenlagen inter- bzw. extrapoliert. Die so hergeleiteten, rein höhenabhängigen Schätzwerte wurden den Stichprobenpunkten zugewiesen und gingen anschließend als unabhängige Variable in das Modell ein.

Im Sinne der Fortführung des Projektes und der bereits vorgestellten Ergebnisse sollte der Faktor Schneehöhe ursprünglich auch in der vorliegenden Arbeit berücksichtigt werden. Unter dem Anspruch, die Schneehöhenwerte an den einzelnen Punkten möglichst zuverlässig zu ermitteln, erfolgte daraufhin eine tiefergehende Auseinandersetzung mit dieser Thematik. Immerhin lagen die wenigen verfügbaren Wetterstationen oft kilometerweit von den Inventurpunkten entfernt. Die hierzu durchgeführte Litera- 
turrecherche zeigte jedoch schnell, dass gerade die Schneehöhen im Gelände diversen Einflussfaktoren unterworfen ist, welche im vorliegenden Fall sämtlich als unbekannt gelten mussten.

Abgesehen von bereits auf lokaler Ebene stark unterschiedlichen Niederschlagsmengen tragen vor allem Wind und Windböen zu einer kaum kalkulierbaren Verteilung der Schneemengen bei. Daneben bestimmen Luv- und Leelage, aber auch Geländeausprägungen die anfallende Schneehöhe. Wie lange und in welcher Beschaffenheit der Schnee vor Ort verweilt, hängt zudem vom lokalen Strahlungsinput durch Hangrichtung und -neigung sowie dem Bewuchs der Fläche ab. Nimmt man alle Faktoren zusammen, lassen sich also nicht einmal annähernd zuverlässige Aussagen über die Schneehöhe oder die Liegedauer des Schnees für konkrete Geländebereiche machen, wenn für diese keinerlei eigene Messwerte vorliegen. Gespräche mit Experten der Bioklimatologie bestätigten diese Einschätzung. Auf die Inter- und Extrapolation gemittelter Werte zur Schaffung einer weiteren unabhängigen Modellvariable wurde deshalb am Ende aller Bemühungen verzichtet.

\subsection{Betrachtung der eigenen Analyse}

\subsubsection{Bewertung der Datengrundlage}

Grundsätzlich muss diskutiert werden, ob die vorhandene Datengrundlage zur Beantwortung der übermittelten Fragestellung überhaupt geeignet ist. Im Kern geht es immerhin um nicht weniger, als um die Suche nach kausalen Zusammenhängen zwischen einer Vielzahl möglicher Einflussfaktoren und der Intensität und Verteilung von Schälereignissen durch freilebendes und nicht telemetriertes Rotwild. Allein die Ausgangslage lässt also so gut wie alles offen. Dass bei einer solchen Untersuchung ohnehin nur eine Auswahl angenommener Einflussfaktoren betrachtet werden kann, während andere Faktoren unberücksichtigt bleiben, gehört zu den grundsätzlichen Rahmenbedingungen. Die Hauptursache für die kritische Bewertung der vorliegenden Datenbasis liegt indes in der unterschiedlichen Herkunft der Daten selbst und ist dem Umstand geschuldet, dass weder die Parameter für die abhängige noch die für die unabhängigen Variablen explizit zum Zweck einer solchen Untersuchung erhoben worden sind. So mussten die in den Modellen verwendeten Parameter zunächst durch studienfremdes Datenmaterial hergeleitet und den mutmaßlichen Verhältnissen an den Stichprobenpunkten angenähert werden. Anschauliches Beispiel hierfür sind die Informationen aus 
den Forsteinrichtungswerken. Auch größte Akribie und Gewissenhaftigkeit bei der Aufbereitung von Daten können den Nachteil ihrer ursprünglichen Fremdbestimmung nicht ausgleichen. Gerade die hierbei häufig unumgängliche Beschränkung auf Mittelwerte über große Flächeneinheiten muss für eine solche Fragestellung unbefriedigend bleiben, da hierdurch diverse Stichprobenpunkte unter einer einzigen Faktorausprägung subsummiert werden. Dies geschieht, obwohl die anschließende georeferenzierte Verschneidung der Parameter mit den einzelnen Stichprobenpunkten im GIS von ihrem Potential her wieder ein sehr hohes Maß an Genauigkeit zuließe. Die Parametermatrix, welche als Datengrundlage schließlich in Analysemodelle eingeht, beinhaltet also trotz rechnerischer Genauigkeit stets einen nicht zu kompensierenden Grad an Unschärfe.

Neben diesem Problem der Unschärfe gilt es, einen weiteren Aspekt zu berücksichtigen, welcher sich ebenfalls nicht auf den ersten Blick offenbart. So ermöglichen umfangreiche Daten zwar grundsätzlich, hieraus wieder neue Parameter zu generieren, etwa durch die Bildung von Quotienten. Diese Option kann aber auch zur Verleitung werden. Denn die zuvor skizzierten Schwierigkeiten bei der Verwendung von Mittelwerten können sich bei deren weiteren Verrechnung schnell potenzieren. Zudem sollte man sich bewusst sein, dass nicht jeder Wert, der mathematisch herzuleiten ist, sich vor Ort in gleicher Form auch als Zähl- oder Messwert ergeben hätte. Die Zuverlässigkeit und inhaltliche Aussagekraft eines derartig generierten Parameters sollte also stets hinterfragt werden. Von den anfänglich auf Basis der Forsteinrichtungswerke hergeleiteten Parametern blieb so im Laufe der eigenen kritischen Prüfung nur ein Teil für die Modellrechnung bestehen.

Die gleichzeitige Erhebung weiterer Parameter direkt am aufgenommenen Baum, schon während der Schälschadensinventur, wäre deshalb wohl von Vorteil gewesen. Zusammen mit der abhängigen Variablen „Schälvorkommen“ hätten dann dem Stichprobenpunkt unmittelbar zuordenbare unabhängige Variablen zur Verfügung gestanden. Auf den Einzelbaum bezogen sind dies vor allem Messdaten zum Brusthöhendurchmesser und Werte der Astigkeit, wie z.B. der mittlere Abstand der Astquirle am Stamm Auf die nähere Umgebung des Stichprobenpunktes bezogen könnten bestandesbeschreibende Merkmale gemessen oder vor Ort visuell eingeschätzt werden, wie zum Beispiel der Kronenschlussgrad oder die Stufigkeit des Bestandes. Der Weg, diese und andere Parameter über die Forsteinrichtungsdaten zu ermitteln und dann den Stichprobenpunkten zuzuordnen, kann nach den obigen Feststellungen hingegen nur bedingt als zielführend bewertet werden. Die ohnehin zumeist über eine große Fläche gemittelten Mess- und 
Schätzwerte des Einrichtungswerkes würden hierdurch mehreren Stichprobenpunkten in gleicher Ausprägung zugewiesen werden, ohne zwischen den lokalen Gegebenheiten differenzieren zu können. Aussagen über die Beschaffenheit der konkret in die Stichprobe aufgenommenen Bäume entbehrten umso mehr einer robusten Grundlage.

Überhaupt stellt sich die Frage, ob nicht ein Stichprobendesign auf Basis von Probekreisen geeigneter für eine solche Analyse von Schälschäden wäre. Anstatt mittels Klumpenstichprobe stets 18 Bäume je Stichprobenpunkt aufzunehmen, ließe die Verwendung von definierten Probekreisen unter anderem Aussagen zu Dichteverhältnissen des Baumbestandes im Punkt zu. Auch weitere Merkmale zur Beschreibung der lokalen Situation ließen sich so berücksichtigen. Über die Dokumentation von Baumarten, Brusthöhendurchmesser und Baumhöhe könnten diese Werte unmittelbar in Beziehung zur Schälschädigung gesetzt werden. Voraussetzung hierfür wäre allerdings wiederum eine Auswertung, bei der die abhängige Variable nicht binär codiert, sondern als relativer Anteil in das Modell eingeht. Bei der vorliegenden weiterführenden Analyse im GLM ist diese Variante ja bereits gewählt worden.

So könnten dann nicht nur junge, zuvor als schälgefährdet eingestufte Fichtenbestände in die Analyse eingehen, sondern alle zufällig über das Raster ausgewählten Stichprobenpunkte. Im Datenpool wäre somit eine breitere Variation der lokalen Bedingungen abgebildet. Die flächenscharf an den jeweiligen Stichprobenpunkten ermittelten Parameterwerte böten dann die Grundlage für differenziertere Aussagen. Denn auch in älteren Beständen finden sich Bäume in schälfähigen Dimensionen. Die Tendenz der Forstbetriebe, Altersklassenwald in reicher strukturierten Dauerwald zu überführen, verstärkt diesen Effekt durch die dann breitere Varianz der Baumdimensionen in den Beständen. Auch die Analyse des Schälaufkommens über alle Baumarten hinweg erscheint in diesem Zusammenhang sinnvoll, um auch hier den Informationsverlust zu minimieren. Eine derartige Untersuchung nehme zwar an Komplexität zu, böte aber auch ein vollständigeres Bild der tatsächlichen Schälsituation vor Ort.

Im Zuge der Schälschadensinventur selbst sind keine geografischen Parameter aufgenommen worden. Durch das Stichprobendesign und die rasterbasierte Auswahl der Stichprobenpunkte lagen jedoch deren Koordinaten als Rechts- und Hochwert im GaussKrüger-System vor. Mit der Verwendung eines digitalen Geländemodells war es somit möglich, topografische Kenngrößen herzuleiten. Neben der Höhe über Normalnull insbesondere auch solche, die aufgrund ihrer Komplexität ohne computergestützte Mo- 
delle nicht zu erheben gewesen wären, wie zum Beispiel der Grad der Exponiertheit im Gelände.

Nun wird ein digitales Geländemodell sicher nicht im Rahmen jeder Studie als Instrument zur Verfügung stehen. Allein die Georeferenzierung von Daten in einem GIS bietet jedoch schon Vorteile, die in diesem Zusammenhang stärker genutzt werden sollten. Abseits der Herleitung topografischer Parameter beschränkte sich die Anwendung des GIS in der vorliegenden Arbeit auf die Verschneidung der einzelnen Datensets. Gerade bei einer Untersuchung auf Landschaftsebene könnte aber darüber hinaus auch die Berücksichtigung von Nachbarschaftsbeziehungen der Stichprobenpunkte zueinander aufschlussreich sein. Insbesondere deshalb, weil die abhängige Variable ja auch das Raumverhalten des Rotwildes widerspiegelt.

Möglich wäre, dass damit räumliche Abhängigkeiten aufgedeckt werden und sich das Erklärungspotential von Modellen durch diese Aspekte erhöht. In der vorliegenden Untersuchung könnten signifikante, jedoch zwischen den einzelnen Modellen widersprüchliche Effektausprägungen der untersuchten Variablen in dieser Assoziation ihre Ursache haben. Allerdings kommen hierfür natürlich auch andere, nicht im Modell berücksichtigte Parameter infrage. Für weiterführende Studien empfiehlt es sich deshalb generell, stärker auf räumliche Beziehungen einzugehen, um in diesem Bereich mehr Klarheit zu erlangen.

Das Bestreben, die tatsächlichen Verhältnisse am Stichprobenpunkt möglichst realitätsnah im Modell abzubilden, wird indes auch mit großem technischen Aufwand immer Einschränkungen unterworfen bleiben. So stößt auch ein digitales Geländemodell an seine Grenzen, wenn beispielsweise strukturreiche und noch dazu dynamische Komponenten, wie etwa die lokale Vegetation, als dessen Eingangsgröße erfasst werden müssten. Es wären Wachstumsmodelle im Hintergrund nötig, denen ihrerseits äußerst umfangreiche Messdaten zugrunde liegen müssten. Ein Aufwand, der angesichts der Fragestellung und auch der zu erwartenden Erkenntnisse wohl selten zu rechtfertigen wäre. Bei der Berechnung der hier verwendeten Strahlungs- und Exponiertheitsparametern blieb die Vegetation aus Mangel an Alternativen gänzlich unberücksichtigt. Um die tatsächlichen Strahlungsverhältnisse am Waldboden abzubilden, wäre diese Präzisierung allerdings durchaus von Belang gewesen, denn die hierüber klassifizierte Variable zielt ja konkret auf das für das Rotwild nutzbare Strahlungsangebot am Boden. Für künftige Untersuchungen gilt es also, hierfür den optimalen Kompromiss zu finden. Eine ergänzende Einschätzung vor Ort, welche während der Inventur durch- 
geführt werden kann, wäre vielleicht auch hier ein geeignetes Mittel, um die errechneten Werte mit der Gesamtsituation am Standort in Beziehung zu bringen.

Die Beschäftigung mit den Abschusszahlen beim Rotwild und damit das Ringen um eine Quantifizierung der Rotwilddichte im Raum zählt ebenso zu dem übergeordneten Ziel, die Situation am Stichprobenpunkt möglichst realitätsnah im Modell abzubilden. Die in der Arbeit verwendeten Daten der Rotwilderlegungen bieten räumliche und zeitliche Informationen. Sie liegen abteilungsscharf und auf den konkreten Erlegungstag datiert vor. Beim räumlichen Bezug erschwerten Strukturreformen innerhalb der Forstverwaltung und der -betriebe die verlässliche Zuordnung der Daten über einen längeren Betrachtungszeitraum. Diese Aufgabe wurde mittels digitaler und analoger Karten zwar zeitaufwendig, im Ergebnis jedoch zufriedenstellend gelöst. Grundsätzlich stellt sich aber die Frage, mit welchem Grad an Genauigkeit die räumlichen Informationen in die Analyse eingehen können und sollen. Dabei ist zu bedenken, dass die forstliche Abteilung, an welche der Datensatz der Erlegung gebunden ist, in ihrer Größe und Ausprägung variiert. Der Jagderfolg, und damit die Erlegungen selbst, hängt dabei häufig von der dort vorhandenen jagdlichen Infrastruktur, sprich: den Ansitzeinrichtungen, ausgebrachten Salzlecksteinen und gegebenen (Zwangs-)Wechseln des Wildes ab. Diese Faktoren beeinflussen das Ergebnis und schwächen so die Indikation für die Rotwilddichte in der Fläche. Weil keine Abteilung isoliert dasteht und es Grenzeffekte gibt, müssten außerdem die Nachbarschaftsbeziehungen der Stichprobenpunkte abteilungsübergreifend berücksichtigt werden.

In der vorliegenden Arbeit wurde als Bezugsmaßstab das jeweilige Forstrevier gewählt, um all diese Faktoren weitestgehend zu egalisieren. Dennoch bleiben vermutlich infrastrukturelle und auch auf den jeweiligen Jagdausübenden bezogene Unterschiede in unbekanntem Ausmaß bestehen, welche den Rückschluss von erlegtem auf insgesamt vorkommendes Wild beeinflussen. Aus dieser Betrachtungsweise heraus böten andere Arten der Dichteschätzungen, wie zum Beispiel Methoden der Losungszählung, möglicherweise Vorteile.

\subsubsection{Bewertung der Ergebnisse}

Wie bereits dargelegt, fällt die Beurteilung der Datengrundlage insgesamt skeptisch aus. Die entsprechend kritische Herangehensweise an die Analyse erfolgte deshalb auf verschiedenen Wegen. Wie sind die unter diesen Umständen generierten Ergebnisse nun zu bewerten? Zunächst einmal steht eine grundsätzliche Entscheidung an. Sollen die 
einzelnen Modelle hinsichtlich ihrer Modellgüte und des Grades ihrer Anpassung im Detail diskutiert werden? Die Antwort lautet an dieser Stelle nein, denn dann ginge es wieder „nur“ darum, ein geeignetes Modell vorzuschlagen, mit welchem sich Voraussagen auf künftige Schälereignisse treffen ließen. Gerade dieser Suggestion einer zuverlässigen Schätzung soll diese Arbeit jedoch nicht erliegen. Ziel der vorliegenden Untersuchung ist eben nicht, absolute Aussagen über kausale Zusammenhänge zu treffen, welche durch mehr oder minder robuste Modellparameter gestützt werden. Vielmehr ist durch den Verlauf der Arbeit deutlich geworden, wie unterschiedlich eben diese Aussagen je nach Modell und Effektparameter ausfallen können und wie wenig sicher damit deren Effektstärke und sogar -richtung mitunter ist. Was bleibt, ist also die vorsichtige Auseinandersetzung mit den durch die Analysen aufgezeigten Tendenzen und deren Einordnung in die Ergebnisse bisheriger Forschungsarbeiten. Unter Berücksichtigung der Physiologie und des Verhaltens des Rotwildes lässt sich dabei natürlich über mögliche kausale Zusammenhänge spekulieren. Dieser Ansatz soll auch im Folgenden genutzt werden, um sich den inhaltlichen Aussagen dieser und anderer Forschungsarbeiten zu nähern. Es soll in diesem Kontext aber ganz explizit nicht der Anspruch auf eine allgemeingültige qualitative oder gar quantitative Verbindlichkeit der eigenen Ergebnisse erhoben werden.

\section{Populationsdichte}

Bei wissenschaftlichen Untersuchungen zum Aufkommen und zu der Verteilung von Schälschäden steht die Frage der Populationsdichte des Rotwildes vielfach im Vordergrund, wobei in der Literatur inhaltlich zwischen Arbeiten über eine wirtschaftlich tragbare Wilddichte (Müller 1963, Speidel 1975, MaYer 1975, Wagenknecht 1994) und solchen über eine ökologisch tragbare Wilddichte (MAYER 1973, BuRSCHEL 1975, BuRSCHEL 1993) unterschieden wird (UECKERMAnN 1960). Vor allem der Begriff der wirtschaftlich tragbaren Dichte wird laut PRIEN (1997) allerdings weder in seiner Definition noch in seiner Interpretation einheitlich angewendet. Beiden Betrachtungsweisen ist die Frage nach der tatsächlichen Populationsgröße und vor allem der Populationsdichte gemein. Außerdem unterstellen viele Autoren einen direkten Zusammenhang zwischen der Populationsdichte und der Schälschädigungsrate (z.B. Ueckermann 1960, Szukiel 1978, Kaji et Al. 1984, Kraus 1987, Maizeret \& Ballon 1990, Verheyden et AL. 2006, Klemen ET AL. 2008), welcher in einigen Fällen auch statistisch nachgewiesen werden konnte (UECKERMANN 1956, KÖNIG 
1969, Pheiffer \& Hartfield 1984, Randveer \& Heikkilä 1996, Yokoyama 2001).

Die Tatsache, dass die Daten anderer Autoren keine solchen Schlüsse zuließen (GERKe 1980, Müller 1985, Welch et Al. 1987, Saint-Andrieux \& Klein 1995, VÖLK 1997, VÖLK 1998, VÖLK 1999, JALKANEN 2001), weist jedoch auf die Schwierigkeiten dieses Vorhabens hin. Denn gerade im Wald gilt die Schätzung der Populationsgröß $\oint^{1}$ von Wildtieren und deren lokale Verteilung als äußerst schwieriges Unterfangen (Andersen 1953, Gill 1990, Smart et Al. 2004) und nicht selten muss die Verlässlichkeit der über verschiedene Verfahren geschätzten Daten in Zweifel gezogen werden (Gill 1992, Mотта 1997).

Meist handelt es sich bei den Schätzungen ja um eine Momentaufnahme, deren Fortschreibung oder Rückberechnung in der Regel über die unterstellte Reproduktionsrate und Mortalität der Art erfolgt. Aussagen über Populationen erfordern aber streng genommen auch die Betrachtung des Migrationsverhaltens, wobei für das potentielle Maß der Migration einer Art deren individuelle Mobilität ausschlaggebend ist. Diese wiederum kann ermittelt werden, indem man die Wahrscheinlichkeit der Überwindung von Distanzen berechnet (AMLER ET AL. 1999) und dabei die Orientierung der Tiere an Kulissen (Wolf 1994, Newmark \& RickART 2012) oder Korridorstrukturen (OpITz ET AL. 1998) in der Landschaft berücksichtigt. So existieren in der allgemeinen Ökologie inzwischen verschiedene Methoden zur Quantifizierung von Migration (Brownie et Al. 1993, Settele 1998).

Im Zusammenhang mit der räumlichen Verteilung von Rotwild, insbesondere mit Blick auf die Wahrscheinlichkeitsberechnung von Schälschäden, finden solche Methoden jedoch keine Anwendung, auch in der vorliegenden Arbeit nicht. Sie würden wohl auch über das hinnehmbare Maß an Komplexität solcher Studien hinausgehen. Klar ist aber, dass die angenommenen Populationsgrößen stets nur Annäherungen an die Realität darstellen und dass die unterstellten Aufenthaltsschwerpunkte das tatsächliche Verteilungsmuster der Individuen in Raum und Zeit nur sehr beschränkt abbilden können. Jagdstreckendaten ergeben also allenfalls einen ganz kleinen Einblick in das, was sich als komplexes System innerhalb und zwischen den Habitaten im Jahreslauf ereignet.

\footnotetext{
${ }^{1}$ Als Population wird eine Gruppe von Individuen bezeichnet, die sich untereinander kreuzen, wobei dieser Begriff unabhängig von der Größe der Population ist. So kann diese von einigen wenigen Individuen gebildet werden oder aber eine unüberschaubare Vielzahl von Mitgliedern in sich vereinen. Von höchster Relevanz aber ist die Fähigkeit, Nachkommen zu produzieren (PRIMACK 1995).
} 
So sind nach BüTZLER 2001 die Äsungsverhältnisse im Laufe des Jahres dafür entscheidend, wie sich die Individuenverteilung und damit die Dichte im Rotwildhabitat darstellen. Allerdings nur unter der Prämisse natürlicher Bedingungen. Habitate spielen für die Gestaltung von Lebenszyklen generell eine entscheidende Rolle, wobei in der Wissenschaft eine Einteilung in für die Population günstige bis ungünstige Habitate üblich ist (BEGON ET AL. 1991). In der Auseinandersetzung mit der Arten-ArealBeziehung findet sich im Bereich der Ökologie wiederum eine Vielzahl von Ansätzen zur Landschaftsqualifizierung, um hierüber die Eignung von Habitaten zu modellieren (z.B. bei TJøRVE 2002). Diese Betrachtungsweise unterscheidet sich also schon im Grundsatz von der hier durchgeführten Analyse der Jagdstreckendaten.

Dem geschilderten Ansatz über die Qualifizierung von Habitaten ist jedoch VoSPERNIK (2006) in ihrer Arbeit zur Analyse von Schälschäden nachgegangen. Im Gegensatz zu den für Dichteschätzungen von Rotwild üblicherweise herangezogenen Losungskartierungen, Abschusszahlen oder Sichtbeobachtungen teilte sie das Untersuchungsgebiet in Regionen unterschiedlicher Habitateignung für Rotwild ein. Dabei zeigten sich hohe Schälraten in jenen Regionen, die als geeignet klassifizierten worden waren, während Regionen, die als weniger geeignet galten, auch weniger Schälschäden aufwiesen. Auch VosperniK sieht in den Ergebnissen einen eindeutigen Zusammenhang zwischen einer hohen Rotwilddichte einerseits und einem hohen Aufkommen an Schälschäden andererseits bestätigt. Von einer kausalen Beziehung der Populationsdichte mit der Schädigungsrate geht sie grundsätzlich aus. Sie weist darauf hin, dass derartige Zusammenhänge in der Literatur immer dann statistisch eindeutig zu belegen waren, wenn sich die Populationsgröße des Rotwildes im Untersuchungsgebiet innerhalb kurzer Zeit deutlich veränderte. Als Beispiel führt sie die Arbeit von MoтTA (1997) an, in der durch die Wiederansiedlung von Rotwild in den Italienischen Alpen ein entsprechend starker Anstieg der Populationsgröße, ausgehend von nur wenigen Tieren, stattfand und dokumentiert wurde. So schreibt sie den fehlenden Nachweis eines Zusammenhangs in den Arbeiten anderer Autoren den geringeren Schwankungen der Populationsgröße des dortigen Rotwildes zu. Hierin sehen auch SAINT-AnDRIEUx \& KLEIN (1995) den fehlenden Nachweis eines statistisch signifikanten Zusammenhangs in ihren Untersuchungen begründet.

Offenbar spielt hierbei jedoch auch die Bezugsgröße des Untersuchungsgebietes eine Rolle, wie eine Studie zum Verbiss durch Elche zeigt. Obwohl die dortige Veränderung der Schädigungsrate auf großer Fläche mit einer Schwankung der Elchpopulation in 
Zusammenhang gebracht werden konnte, ließ sich auf lokaler Ebene zeitgleich keine Verbindung dieser beiden Größen herstellen (HöRNBERG 2001). Stattdessen wurden die klimatischen Bedingungen, die lokalen Habitatstrukturen und die damit verbundene Verfügbarkeit von Nahrung als Ursachen für die kleinflächig dokumentierten Schwankungen herangezogen. Insgesamt muss der Zusammenhang von Populationsdichte und Einfluss auf die Vegetation durch Schäle oder Verbiss, so trivial er zunächst auch gelten mag, in seinem Nachweis also als äußerst komplex angesehen werden.

\section{Welche Rolle spielt die Feindvermeidung?}

Unmittelbar mit der Betrachtung der Populationsdichte und insbesondere der Habitatnutzung verknüpft ist die Feindvermeidung des Rotwildes, denn dieser Aspekt tritt als zusätzliche Störgröße auf. In vielen Bereichen zeigt sich, dass die Feindvermeidung für das Rotwild im Aktivitätsmuster Priorität hat und so ist letztlich auch das Wiederkauen als eine Anpassungsstrategie hieran zu verstehen (HEDIGER 1946). Es reduziert die Zeit der aktiven Nahrungsaufnahme auf ein Mindestmaß. Die Wahrscheinlichkeit, beim ruhigen Wiederkauen von Predatoren entdeckt zu werden, ist schließlich deutlich geringer als während der Aktivitätsphasen. Dank dieser insgesamt 5 bis 6 Stunden andauernden Zeit des erneuten Zerkauens der Nahrung beträgt die verbleibende Äsungsdauer nur noch 7 bis 10 Stunden täglich (Bubenik \& Lochmann 1956, BubeniK 1965). Den Faktoren, die in dieser Zeit Einfluss auf die Standortswahl des Rotwildes und damit auch auf die Schälschäden nehmen könnten, können in dieser Untersuchung indirekt sowohl Jagd- als auch Besucherdruck (ausschließlich im Harz erhoben) zugeordnet werden. Indirekt deshalb, weil bei der Jagd nicht die Ansitzhäufigkeit, sondern die Zahl der Erlegungen in die Untersuchung eingegangen ist. Insofern dienen die Jagdstreckendaten vielmehr als Indikator für die angenommene lokale Populationsdichte und sagen nichts darüber aus, wie hoch der örtliche Druck oder Stress durch den jagenden Menschen in Form von aktiven Pirschgängen tatsächlich gewesen ist.

Welche Rolle wiederum genügend Deckung bei der Feindvermeidung spielt und wie diese vom Rotwild in ihrer Wirksamkeit beurteilt wird, zeigen andere Schälschadensuntersuchungen auf neu bestockten Kalamitätsflächen. Demnach ist insbesodere im Wald die Vegetationshöhe entscheidend für das Sicherheitsgefühl des Rotwildes. Flächen mit einem ausreichenden Angebot an Deckung wurden häufiger vom Rotwild aufgesucht und die darin befindlichen Bäume entsprechend intensiv geschädigt. Eine Bevorzugung von Baumbestockungen aus Naturverjüngung gegenüber solchen aus Pflanzung wur- 
de hingegen nicht festgestellt und auch das Alter der Bäume musste innerhalb einer gewissen Spanne als nachrangig für die Gefährdung durch Schäle angesehen werden. Viel wichtiger war stattdessen die Höhe der Bäume, wobei auch diese sich nur in einem Bereich von bis zu 2 Metern maßgeblich auf das Ergebnis auswirkte, um im Bereich darüber offenbar konstant als „günstig“ bewertet zu werden (BORKOWSKI \& UKALSKI 2012). Bei einer Schulterhöhe des Rotwildes zwischen 110 und 130 cm (BÜTZLER 2001, bezogen auf Deutschland) ist diese Bewertung aus Sicht der sich darin verbergenden Tiere durchaus nachvollziehbar.

Die im Gelände vorhandene Deckung konnte in Hinblick auf diese Fragestellung nicht in ausreichender Form aus den Bestandesdaten der Forsteinrichtungswerke abgeleitet werden. Als Indiz innerhalb der vorliegenden Arbeit mag man lediglich das in der Analyse festgestellte verringerte Schälrisiko an exponierten Standorten auffassen (Kapitel GLM). Gleichwohl dieser Faktor nichts über die Vegetation am Standort aussagt, bieten im Umkehrschluss wenig exponierte Bereiche im Gelände häufig eine von außen eingeschränkte Einsicht und damit womöglich generell mehr Sichtschutz für das Rotwild. Das Vorhandensein von Deckung wurde in anderen Untersuchungen übrigens auch dann als äußerst wichtiges Kriterium für die Standortwahl des Rotwildes nachgewiesen, wenn große Predatoren gar nicht im Gebiet vorkamen (ADRADOs ET AL. 2008, Borkowski und UKALSKI 2008) oder extrem selten waren (BonENFANT ET AL. 2002), sofern stattdessen der Mensch als Jäger in Erscheinung trat. Welch großen Einfluss der jagende Mensch auf die Wahl des Einstands bei Rotwild haben mag, lassen auch Untersuchungen von Theuerkauf und Rouys (2008) erahnen. Die von ihnen festgestellten Verhaltensanpassungen nordamerikanischer Wapitis (Cervus canadensis) zur Feindvermeidung waren deutlich ausgeprägter, wenn es um den Kontakt zum Menschen ging, als dies in Bezug auf Wölfe (Canis lupus) der Fall war.

Die Auswirkungen der Jagd als Populationsmaß einerseits und als Feindvermeidungsfaktor andererseits könnten einander also durchaus in ihrer Richtung widersprechen. So zeigte sich bei der Untersuchung der Chancenverhältnisse im Harz ein Anstieg des Schälrisikos mit zunehmender Rotwildstrecke. Ähnliche Ergebnisse lieferten die Daten aus dem Solling. Dort kehrte sich dieser statistische Zusammenhang an der Spitze jedoch um. Ab einem sehr hohen Jagdstreckenergebnis sank das Schälrisiko in dem dortigen Gebiet wieder. Unterstellt man, dass nur dort, wo eine hohe Rotwildpopulationsdichte vorherrscht auch viel erlegt wird, bzw. überhaupt erlegt werden kann, liegt die Annahme der Jagdstrecke als Indikator hierfür nahe. In den Modellen der logis- 
tischen Regression konnten im Harz hingegen keine und im Solling sogar gegenteilige Aussagen hierüber generiert werden. Demnach sinkt dort das Schälrisiko mit Ansteigen der erzielten Rotwildstrecke. Würde man also ausschließlich auf die Ergebnisse aus dem Solling zurückgreifen können, läge die Vermutung nahe, die Jagdstrecke spiegele zugleich den ausgeübten Jagddruck und führte so zu einem Vergrämungseffekt auf den jagdlich intensiv genutzten Flächen. Mit der Folge, dass das Schälrisiko dort gegenüber den restlichen Flächen zurückginge.

Derselben Annahme von Vergrämungseffekten durch den Menschen folgen auch Untersuchungen, die diesen Faktor nicht direkt untersuchen. So wird eine höhere Frequenzierung tiefer gelegener Geländebereiche durch den Menschen bei DeBELJAK ET AL. (2001) als Erklärung dafür angenommen, dass sich das Rotwild vornehmlich in höheren Lagen aufhält. In manchen Untersuchungen geht die Zahl der Erholungssuchenden hingegen direkt als Faktor in die Analyse ein. Dennoch kann diese Annahme auch dort häufig nicht durch statistisch eindeutige Ergebnisse belegt werden (im Solling zum Beispiel bei Marwede \& SchneIder 2004, beim Thema Verbiss bei Sorges 1999). Auch in der vorliegenden Arbeit wurde dieser Faktor für den Harz untersucht, ergab aber ebenfalls keinen statistisch nachweisbaren Zusammenhang mit der Höhe der Schälrate. Inwieweit dieser fehlende Nachweis als nicht vorhandener Zusammenhang des Faktors mit der Schälrate gewertet werden kann, bleibt leider offen. Die vorliegende Quantifizierung der Wanderwege nach Besucherintensität und die anschließende räumliche Verknüpfung mit den Stichprobenpunkten der Umgebung führte zumindest zu keinem klaren Ergebnis. Auch hier sollten künftige Untersuchungen gegebenenfalls eigene Kriterien definieren und in der Fläche bewerten, um zu konkreteren Aussagen zu gelangen. Die Tatsache, dass das Vorhandensein vorangegangener Schälschaden am Stichprobenpunkt - in dieser Untersuchung durch die Parameter Altschäle und Kontinuität einbezogen- sehr eng mit dem Auftreten erneuter Schäle am Punkt einhergeht, lässt jedenfalls vermuten, dass sich die Bedingungen an den schälintensiven Orten grundsätzlich von anderen Standorten unterscheiden. Der Umstand verweist somit auf das generelle Problem, dass nicht bekannte oder nicht in dieser Form erhobene, tatsächliche Einflussfaktoren nur mehr oder minder durch die im Modell verwendeten Parameter gestreift werden.

\section{Geländeausprägung: Wettereinfluss oder Feindvermeidung?}

$\mathrm{Zu}$ den untersuchten Umwelteinflüssen zählen auch die lokalen Geländeausprägungen, welche vorrangig auf die damit verbundenen unterschiedlichen Ausprägungen des Wet- 
tergeschehens fokossieren. Für ein homöothermes Tier, dass den Winter über aktiv bleibt, dürften z.B. kleinflächig unterschiedliche Wind- und Temperaturverhältnisse im Lebensraum für den Wärmehaushalt des Körpers durchaus eine Rolle spielen. Insbesondere durch den im Winter reduzierten Stoffwechsel des Rotwildes wird bei dessen Art der Nahrungssuche stets eine energetische Kosten-Nutzen-Abwägung unterstellt (ARnold ET AL. 2004). Durch die Nahrungssuche an windexponierten oder tief verschneiten Orten mehr Energie zu verlieren als durch die dortige Nahrungsaufnahme hinzugewonnen wird, gelte es also aus Sicht des Rotwildes zu vermeiden. Auch auf weite Distanzen zwischen Ruhe- und Äsungsort wird aus diesem Grund im Winter, wenn möglich, verzichtet. So weisen Studien zur Habitatnutzung von Rotwild (JERINA 2006) darauf hin, dass sich die Tiere im Winter bevorzugt im Randbereich des Waldes aufhalten. Der regelmäßige Wechsel zwischen dichtem Tageseinstand und Äsung im offenen Gelände verlange somit nur kurze Wege vom Rotwild, was sich nach KLEMEN ET AL. (2008) mit dessen eigenen Ergebnissen deckt, wonach Schälschaden im Winter vermehrt im Waldrandbereich (bis 800 Meter ins Waldesinnere hinein) auftreten. Dass die klimatischen Bedingungen insgesamt einen nicht unerheblichen Einfluss auf die Konstitution des Rotwildes nehmen, zeigt eine hierauf ausgerichtete Studie aus Norwegen (Mysterud ET AL. 2007). Eine Verhaltensanpassung an die vorherrschenden mikroklimatischen Verhältnisse ist also naheliegend.

Der Aspekt des Energiehaushalts beim Rotwild wird auch von ScHmidT (1993) und ARNOLD ET AL. (2004) angeführt, die Schälschäden als Indikator für sonnige Waldstandorte mit thermal begünstigter Umgebung heranziehen. Ihren Ergebnissen zur Folge ist die Schälwahrscheinlichkeit an Orten mit lang anhaltend hohem Strahlungspotential höher als in weniger strahlungsexponierten Bereichen. SCHMIDT verweist in diesem Zusammenhang auch auf die Arbeit von Skovlin (1982), der von einer vergleichbar selektiven Habitatnutzung des Wapitis (Cervus canadensis) in Nordamerika berichtet. Nächtliche kalte Fallwinde werden dort offenbar von den Hirschen gemieden, indem sie die betroffenen Tallagen gezielt umgehen.

Auch die vorliegenden Untersuchungen legen die Schlussfolgerung nahe, dass sich das Rotwild im Winter bevorzugt an strahlungsintensiven Orten aufhält und die dortige Vegetation somit einem stärkeren Einfluss durch diese Pflanzenfresser unterliegt. Standorte mit hohem Strahlungsinput im Januar zeigten erhöhte Schälschädigungsraten. Auch in den Untersuchungen von Gossow (1988) finden sich Hinweise darauf, dass windgeschützte, sonnige Bereiche bevorzugt vom Rotwild aufgesucht werden und sich 
die dortige Schälrate entsprechend erhöht. Seine Annahmen werden durch spätere Beobachtungen gestützt, wonach Rotwild gezielt südlich exponierte Hanglagen als Ruhebereich aufsucht (Adrados 2008). Eine Abhängigkeit der Schälrate von klar determinierten Expositionen der untersuchten Hänge kann in den eigenen Untersuchungen indes nicht festgestellt werden. Wohl aber wird der Faktor Exposition insgesamt häufig als erklärende Variable durch die Modelle vorgeschlagen, ohne dass sich aus den einzelnen Klassen eine generelle Bevorzugung bestimmter Himmelsrichtungen durch das Rotwild ableiten ließe.

Die mancherorts festgestellte Abhängigkeit der Schälintensität zur Höhenlage des Stichprobenpunktes (TAKEUCHI ET AL. 2011) kann anhand der eigenen Daten ebenfalls nicht nachvollzogen werden, doch zeigen sich teilweise erhöhte Schälraten mit zunehmender Hangneigung des Geländes. Die Gründe hierfür mögen wiederum vielfältig sein. Im Sinne des hier diskutierten Zusammenhangs mit klimatischen Bedingungen mag man mutmaßen, dass die Schneelage an steileren Hängen durch geringere Auflage und zusätzlichen Windeinfluss reduziert ist und dadurch einen Zugang zur Äsung erleichtert. Auf dieser Argumentationslinie irritieren jedoch die geringeren Schälquoten an exponierten Geländestellen, bei denen man einen vergleichbaren Effekt durch den Wind unterstellen könnte. Steilere Hangpartien könnten aber auch dem Sicherheitsbedürfnis des Rotwildes entgegenkommen, da das Gelände unzugänglicher ist und somit weniger vom Menschen frequentiert wird. Vermutlich spielen mehrere Faktoren in die Standortwahl hinein und die Kombination aus Feindvermeidung, Nahrungssuche und Wetterschutz wechselt zeitlich in ihren Einflussgrößen. Dass den Geländeparametern hierbei grundsätzlich eine wichtige Rolle als erklärende Variablen zukommt, lassen die im Vergleich zu den anderen Parametergruppen recht starken Effekte innerhalb der durchgeführten Untersuchungen vermuten. Die allgemein robusteren Ergebnisse der Harzdaten mögen in der dort insgesamt höheren Reliefenergie begründet liegen. Für verlässlichere Aussagen sind aber wohl dennoch gezieltere Studien nötig, deren Datenerfassung dann speziell auf diese Fragestellung abgestimmt sein sollte.

\section{Bestandesfaktoren}

In die vorliegenden Untersuchungen gingen auch Informationen über die Beschaffenheit des jeweiligen Bestandes der Stichprobenpunkte ein. Als Datengrundlage dienten die Erhebungen der Forsteinrichtungswerke. Auch diverse internationale Studien berücksichtigen solche Bestandesparameter als mögliche Einflussfaktoren auf das Vor- 
kommen von Schäl- oder auch Verbissschäden. So konnten DANELL ET AL. (1991) der Wuchskraft des Standortes eine lenkende Wirkung bei der Konzentration des Verbissgeschehens durch Elche zusprechen. Auch SAint-Andrieux \& Klein (1995) nehmen an, dass Strukturen, wie etwa die mittlere Bestandesgröße oder die Baumartenvielfalt, Einfluss auf die Standortwahl des Rotwildes und damit auf die Intensität von Schälereignissen nehmen könnten. Ohne einen direkten Nachweis hierfür aus den erhobenen Daten zu haben, vermuten Sie, dass diese Faktoren in den erstellten Rechenmodellen dann - sozusagen unerkannt - den Effekt der Populationsdichte überlagern könnten. Auch GiLL (1992) geht von solchen Verwechslungen der Kausalitäten aus. So waren bereits durch vorangegangene Studien insbesondere in Beständen mit reichhaltigem Unterstand erhöhte Schälschädigungsraten beobachtet worden (z.B. durch MCLNTYRE 1975 und Staines \& Welch 1984). Zu den angeführten Erklärungen für den Einfluss solcher Bestandesstrukturen zählen wiederum der Faktor Feindvermeidung, dank der dort vorhandenen Deckung, sowie der Vorteil eines besseren Nahrungsangebotes.

In der Untersuchung von VosperniK (2006) werden die Art der Baumartenzusammensetzung im Bestand, der Bestockungsgrad, die Baumhöhe und der Stammdurchmesser in Brusthöhe (BHD) als vermeintlich einflussnehmende Faktoren durch das zugrunde gelegte Regressionsmodell erkannt und für dessen Voraussage verwendet. Der Zusammenhang von Schälrate und der Ausprägung von Bestandesparametern konnte im dortigen Modell also statistisch nachgewiesen werden. In welche Richtung sie wirken und von welchen anderen Umständen diese Effekte abhängen, kann allerdings nicht abschließend erklärt werden.

Auch durch die eigenen Analysen zeigt sich, dass gerade in der Parametergruppe der Bestandesdaten nur sehr bedingt Aussagen hinsichtlich ihrer Effektrichtung getroffen werden können. Entsprechend der Vermutung von Saint-Andrieux \& Klein (1995) wurden in der vorliegenden Untersuchung Bestandesgröße und Baumartenanzahl durchaus als Einflussfaktoren ausgewiesen. Die hierzu durchgeführten Regressionsmodelle berücksichtigen die mittlere Bestandesgröße dabei als negativ korrelierten Faktor für die Risikovorhersage. Mit abnehmender Größe der Flächeneinheit nimmt das Schälrisiko demnach zu. In den meisten Fällen wird dieser Parameter jedoch nicht als signifikante erklärende Variable im Modell belassen oder der Zusammenhang ist eher schwach. Auch die Betrachtung der Baumartenvielfalt innerhalb eines Bestandes führt selten zu relevanten Aussagen für die Modelle. Oft ist die Effektrichtung verschie- 
dener Modelle gegensätzlich, wobei sich die beiden Untersuchungsgebiete zusätzlich dahingehend unterscheiden, dass eine erhöhte Baumartenzahl der Umgebung im Solling eher negativ mit der Schälrate korreliert, während im Harz ein positiver Zusammenhang ausgewiesen wird. Bedenken muss man an dieser Stelle, dass ausschließlich Winterschälschäden an Fichte berücksichtigt werden. Über die Höhe des gesamten Schälaufkommens, z.B. an Buche, wird keine Aussage getroffen. Außerdem lässt sich das tatsächliche Äsungsangebot für das Rotwild durch die genutzten Parameter wohl kaum abbilden.

Die Annahme eines Attraktivitätseffektes reich strukturierter Bestände durch die dort vorhandene Äsung, in dessen Folge es zu lokal erhöhten Schälschäden kommt, erlaubt zudem offenbar keinen Umkehrschluss. So nimmt VöLK (1999) für die von ihm gegenteilig gerade in jungen Reinbeständen festgestellten hohen Schälraten einen Zusammenhang mit dem dort ansonsten sehr begrenzten Nahrungsangebot an - verglichen mit eben jenen Beständen, welche ein höheres Potential an Äsungspflanzen aufweisen. Auch in der eigenen Analyse der Chancenverhältnisse wird dieses erhöhte Schälschadensaufkommen in jüngeren Beständen festgestellt. In diesem Kontext könnte sich demnach auch die Art der Waldbewirtschaftung auf die Habitatnutzung des Rotwildes auswirken und hierüber mit den Schälraten in Beziehung gesetzt werden, schlussfolgern HeInzE ET AL. (2011). Vielleicht, so die Vermutung von BoyCE ET AL. (2003), lassen sich der bevorzugte Aufenthaltsort des Rotwildes und die Vielfalt der Vegetation aber auch nur im räumlich großen Maßstab zueinander bringen. Ein Aspekt, der durch die vorliegenden Analyse der Baumartenzahl in gewissem Maße gestützt werden könnte. Zeigt dieser Parameter doch lediglich bei dem größeren Flächenbezug der Abteilungsebene einen, wenn auch uneindeutigen, Effekt und nicht bei der kleiner gewählten Ebene der Unterabteilung. Will man dieser Frage tiefer nachgehen, empfiehlt sich aber die Einbeziehung der Bodenvegetation, um das tatsächlich vorhandene Äsungspotential schärfer quantifizieren zu können.

Abgesehen von der Gesamtvariation an Baumarten innerhalb der Bestände zeigt sich bei der Betrachtung des Fichtenanteils im Stichprobenpunkt selbst ein eindeutiger und vergleichsweise starker Effekt. Demnach ist die Schälschadenswahrscheinlichkeit der im Plot vorhandenen Fichten umso höher, je weniger Fichten insgesamt im Plot vorhanden sind. Auf den Umstand der auschließlichen Berücksichtigung von Schäle an Fichte und die damit verbundenen Einschränkungen in der Analyse wurde im Zusammenhang mit den Baumartenzahlen bereits hingewiesen. Insofern arbeitet man mit einem unvoll- 
ständigen Bild, wenn man Rückschlüsse von Bestandesausprägungen auf das Schälrisiko ziehen möchte. Hierin könnte das uneindeutige Ergebnis bei der Gefährdung von Reinoder Mischbeständen seinen Ursprung haben. Beim Fichtenanteil ist diese Betrachtung dagegen zielgenauer. Nun könnte man aus dem Ergebnis eines zunehmenden Schäldrucks auf immer weniger verbleibende Fichten schlussfolgern, dass beim Rotwild im Winter ein grundsätzlicher Bedarf an Baumrinde besteht. Durch zunehmende Knappheit des Gutes führte dann ein Mangel zu einer erhöhten Konzentration auf die verfügbaren Einheiten. Solange man bei dieser Betrachtung aber die Schälschäden an den restlichen Baumarten im Stichprobenpunkt außen vor lässt, kann diese Vermutung jedoch nicht weiter verifiziert werden. Ebenso wenig, wie sich abschließende Aussagen über den Grund des Bedarfs treffen lassen.

\subsection{Konsequenz und Ausblick}

In der eigenen Untersuchung und in der Literatur wird deutlich, dass das Rotwild offenbar von vielen Umwelteinflüssen in seinem spezifischen Verhalten gelenkt wird, indem es flexibel auf diese Faktoren reagiert. Diese bestimmenden Einflussfaktoren durch eine Studie zu erfassen und in einem Modell als erklärende Variable eindeutig abzugrenzen, ist mit Blick auf die uneinheitlichen Forschungsergebnisse der eigenen und der hierzu einbezogenen Untersuchungen aber wohl die große Herausforderung solcher Vorhaben. Vom Anspruch, die kausalen Zusammenhänge der Schälereignisse erklären zu können und anhand der mutmaßlichen Erkenntnisse Voraussagen für andere Bestände treffen zu können, sollte man besser zunächst abweichen. Um klare Interpretationen der Ergebnisse zu ermöglichen, bedarf es bereits auf der Ebene der rein statistischen Zusammenhänge bereits einer Vielzahl an Vorüberlegungen, um die untersuchten Korrelationen mit der Schäle möglichst eng von weiteren (oft unbekannten) Faktoren abzugrenzen. Es empfiehlt sich aus dieser Warte eine Aufteilung des Gesamtvorhabens auf verschiedene, parallel auf gleicher Fläche stattfindenen Teiluntersuchungen, in denen Einzelaspekten ganz gezielt nachgegangen werden kann. Sicher bieten die Ergebnisse der spezialisierten Studien für sich genommen dann schärfere Aussagen. In den Kontext zu den im selben Stichprobendesign gewonnenen Erkenntnissen der Synchronstudien gestellt, ließen sich die übergeordneten Interpretationen der Ergebnisse zudem vermutlich besser evaluieren. 



\section{Zusammenfassung}

Die vorliegende Arbeit stellt die Fortführung eines Projektes dar, in dem kausale Zusammenhänge zwischen Umweltfaktoren und dem Auftreten von Winterneuschälschäden an Fichten durch Rotwild im Harz und im Solling untersucht werden sollten. Ergänzt um die Analyse und detaillierte Darstellung der Datengrundlage der beiden Untersuchungsgebiete bedient sich diese Arbeit zunächst einfacher statistischer Tests zur Quantifizierung der während drei Inventurjahren mittels Klumpenstichprobe ermittelten Schälschäden. Hierbei werden die Werte mit Jagdstrecken- und Forsteinrichtungsdaten sowie Geländeparametern univariat assoziiert und die jeweiligen Chancenverhältnisse der Parameterklassen ausgewiesen. Im Anschluss werden die potentiellen Einflussfaktoren dann mittels binärer logistischer Regressionen sowie durch generalisierte lineare Modelle auf ihren Effektwert als erklärende Variable in multivariaten Modellen hin untersucht. Für die Durchführung dieser Untersuchung werden zu Beginn entsprechende Arbeitshypothesen aufgestellt.

Die Berechnung der Chancenverhältnisse zeigt einen starken Zusammenhang zwischen vorangegangener Schäle und dem Auftreten neuer Winterschäle am Stichprobenpunkt. Ebenso lassen sich erhöhte Jagdstreckenergebnisse mit vermehrter Neuschäle assoziieren. Während junge Bestände häufiger Schälschäden aufweisen als ältere Bestände, lassen sich in Bezug auf die untersuchten Geländeausprägungen kaum eindeutige Aussagen treffen.

Die Ergebnisse der Regressionsmodelle variieren in ihrem Niveau der Modellanpassung und des Erklärungspotentials und bleiben hinsichtlich ihrer Aussagekraft im Solling gegenüber dem Harz zurück. Je nach Inventurjahr und Gebiet werden unterschiedliche Variablen als Effektparameter ausgewiesenen. Zudem widersprechen sie einander zum Teil in ihrer Effektrichtung. Auch das Vorhandensein von Altschälvorkommen wird nicht in allen Modellen als erklärende Variable hinzugezogen.

Die generalisierten linearen Modelle im Harz weisen für die reliefbasierten Parameter wiederkehrende Effekte. Ungeachtet der variierenden Effektstärke bleibt deren 
Ausprägung über die gesamte Untersuchungsreihe hinweg gleich. So lassen sich mit zunehmender Hangneigung eine Erhöhung und in Abhängigkeit von der Hangrichtung zumindest Unterschiede des Schälrisikos feststellen. Während das Schälrisiko mit zunehmender Exponiertheit des Stichprobenpunktes im Gelände sinkt, weisen Standorte mit einem höheren Strahlungspotential im Januar auch höhere Schälschädigungsraten auf.

Die in dieser Arbeit erlangten Ergebnisse korrespondieren zum Teil mit den Erfahrungen anderer Autoren, zeigen jedoch auch Unterschiede zu deren Ergebnissen. Die widersprüchlichen Effektwerte der eigenen Untersuchungen lassen dabei grundsätzliche Zweifel an der Zuverlässigkeit der durch multivariate Modelle ausgewiesenen kausalen Zusammenhänge aufkommen. Neben einem geeigneten Stichprobendesign werden insbesondere auch eine zielgerichtete Parameterauswahl und -erhebung als entscheidende Faktoren für die spätere statistische Analyse hervorgehoben. Als Voraussetzung für die Schaffung eindeutig interpretierbarer Ergebnisse wird nachfolgenden Arbeiten von der Verschneidung ursprünglich nicht für die Untersuchung bestimmter Daten abgeraten und stattdessen eine studienabhängige Erhebung empfohlen. 


\section{Summary}

The present work represents the continuation of a project that examined the causal relationships between environmental factors and the occurrence of bark stripping on spruce by red deer in the Harz and Solling mountains in Germany. In addition to a detailed representation and analysis of the data set of the two study sites, this study employs various statistical tests to estimate the bark stripping damage identified during three years by means of cluster sampling inventory. For this purpose, values of bark stripping are associated with the red deer hunting bag as well as with forest inventory data and terrain parameters in univariate models to calculate the odds ratios of the respective parameter classes. Subsequently, the potential influence factors are analyzed using binary logistic regression and generalized linear models in order to determine their effect as an explanatory variable in multivariate models. For the implementation of this study, appropriate working hypotheses are established at the beginning.

The calculation of odds ratios yields a strong correlation between previous bark stripping and the emergence of new stripping at the respective sample point. Similarly, a high hunting bag is associated with increased bark stripping. While young forest stands seem to be more susceptible to bark stripping damage than older stands, the analysis of the influence of terrain characteristics does not give a clear picture.

The results of the regression models vary in their level of model fit and their explanatory potential. In general, results for the Solling site are less predictive than models for the Harz site. Depending on the inventory year and area, variables reported as effect parameters change from one model to another. In addition, they partly contradict each other in their effect direction. Also, the presence of previous bark stripping damage is not included as an explanatory variable in all models.

The generalized linear models for the Harz site show recurring effects for topography based parameters. Despite the varying effect size their characteristics over the entire series of observations is the same. Increasing slope inclination and slope aspect are associated with differences in bark stripping risk. While the risk of bark stripping 
decreases with increasing exposure of the sample point, locations with a higher potential radiation in January show higher stripping rates.

The results of this study only partially correspond with the results of other authors. The contradictory effect values of this analysis raise fundamental doubts about the reliability of causal relationships generated by multivariate models. In addition to an appropriate sampling design, especially targeted parameter selection and collection are highlighted as crucial factors for subsequent statistical analysis. Unambiguous results requires data collection customized specifically to the scientific problem rather than the use of data collected for other purposes. 


\section{Anhang}

Tabelle .1: Bestandestypenschlüssel

\begin{tabular}{llll}
\hline BT & Bestandestyp & BT & Bestandestyp \\
\hline 10 & Eiche rein & 52 & Fichte mit Buche \\
12 & Eiche mit Buche & 53 & Fichte mit Edellaubholz \\
13 & Eiche mit Edellaubholz & 54 & Fichte mit Erle \\
16 & Eiche mit Douglasie & 55 & Fichte mit Sitkafichte \\
18 & Eiche mit Lärche & 57 & Fichte mit Kiefer \\
20 & Buche rein & 58 & Fichte mit Lärche \\
22 & Buche mit Bergahorn & 60 & Douglasie rein \\
23 & Buche mit Edellaubholz & 65 & Douglasie mit Fichte \\
25 & Buche mit Fichte & 70 & Kiefer rein \\
28 & Buche mit Lärche & 74 & Kiefer mit Erle \\
29 & Buche mit Pappel & 83 & Lärche mit Edellaubholz \\
32 & Edellaubholz & 84 & Lärche mit Erle \\
43 & Erle/Birke mit Edellaubholz & 85 & Lärche mit Fichte \\
45 & Erle/Birke mit Fichte & 87 & Lärche mit Kiefer \\
48 & Erle/Birke mit Lärche & 93 & Pappel mit Edellaubholz \\
49 & Erle/Birke mit Pappel & 98 & Eibe \\
50 & Fichte rein & 99 & Tanne \\
\hline
\end{tabular}


Tabelle .2: Baumartenschlüssel

\begin{tabular}{|c|c|c|c|c|c|}
\hline Code & Gruppe & Baumart & Code & Gruppe & Baumart \\
\hline 110 & Eiche & Eiche & 430 & Aln & Pappel \\
\hline 111 & Eiche & Stieleiche & 431 & Aln & Aspe \\
\hline 112 & Eiche & Traubeneiche & 432 & Aln & Schwarzpappel \\
\hline 113 & Eiche & Roteiche & 433 & Aln & Graupappel \\
\hline 114 & Eiche & Zerreiche & 434 & Aln & Balsampappel \\
\hline 115 & Eiche & Sudeteneiche & 441 & Aln & Weide \\
\hline 211 & Buche & Buche & 442 & Aln & Roßkastanie \\
\hline 221 & Buche & Hainbuche & 451 & Aln & Eberesche \\
\hline 311 & Alh & Esche & 452 & Aln & Traubenkirsche \\
\hline 320 & Alh & Ahorn & 511 & Fichte & Fichte \\
\hline 321 & Alh & Bergahorn & 512 & Fichte & Sitkafichte \\
\hline 322 & Alh & Spitzahorn & 513 & Fichte & Omorikafichte \\
\hline 323 & Alh & Feldahorn & 514 & Fichte & Stechfichte \\
\hline 330 & Alh & Rüster & 520 & Fichte & Tanne \\
\hline 331 & Alh & Bergrüster & 521 & Fichte & Weißtane \\
\hline 332 & Alh & Flatterrüster & 522 & Fichte & Nordmanntanne \\
\hline 333 & Alh & Feldrüster & 523 & Fichte & Küstentanne \\
\hline 340 & Alh & Linde & 524 & Fichte & Koloradotanne \\
\hline 341 & Alh & Sommerlinde & 525 & Fichte & Edeltanne \\
\hline 342 & Alh & Winterlinde & 526 & Fichte & Japantanne \\
\hline 351 & Alh & Robinie & 527 & Fichte & Lta \\
\hline 352 & Alh & Kastanie & 528 & Fichte & Veitchitanne \\
\hline 353 & Alh & Nussbaum & 529 & Fichte & Pta \\
\hline 354 & Alh & Kirsche & 531 & Fichte & Sicheltanne \\
\hline 355 & Alh & Apfel & 532 & Fichte & Rzyprese \\
\hline 356 & Alh & Birne & 541 & Fichte & Tsuga \\
\hline 357 & Alh & Elsbeere & 542 & Fichte & Thuja \\
\hline 358 & Alh & Speierling & 543 & Fichte & Chamaecyparis \\
\hline 359 & Alh & Mehlbeere & 551 & Fichte & Sequoia giganteum \\
\hline 361 & Alh & Tulpenbaum & 552 & Fichte & $\begin{array}{l}\text { Metasequoia glyptostroboi- } \\
\text { des }\end{array}$ \\
\hline 362 & Alh & Hickory & 561 & Fichte & Eibe \\
\hline 363 & Alh & Platane & 611 & Douglasie & Douglasie \\
\hline 364 & Alh & Mispel & 711 & Kiefer & Kiefer \\
\hline 365 & Alh & Zwetschge & 712 & Kiefer & Schwarzkiefer \\
\hline 410 & Aln & Birke & 713 & Kiefer & Bergkiefer \\
\hline 411 & Aln & Sandbirke & 714 & Kiefer & Bankskiefer \\
\hline 412 & Aln & Moorbirke & 715 & Kiefer & Pechkiefer \\
\hline 413 & Aln & Japanbirke & 716 & Kiefer & Pinus contorta \\
\hline 414 & Aln & Hybridbirke & 731 & Kiefer & Strobe \\
\hline 420 & Aln & Erle & 810 & Lärche & Lärche \\
\hline 421 & Aln & Roterle & 811 & Lärche & Europäische Lärche \\
\hline 422 & Aln & Weißerle & 812 & Lärche & Japanische Lärche \\
\hline \multirow[t]{2}{*}{423} & Aln & Grünerle & 813 & Lärche & Sudetenlärche \\
\hline & & & 814 & Lärche & Hybridlärche \\
\hline
\end{tabular}




\section{Abbildungsverzeichnis}

$2.1 \quad$ Lage der Untersuchungsgebiete Solling und Harz in Deutschland . . . . 12

2.2 Inventurverfahren nach Trisl $\ldots \ldots \ldots$. . . . . . . . . . . . . . . . . . . . 14

2.3 Anteil der Baumarten Fichte und Buche als Einzelbäume an den Inventurpunkten . . . . . . . . . . . . . . . . . 16

$2.4 \quad$ Fichtenanteil innerhalb der Stichprobenpunkte . . . . . . . . . . . . . 16

2.5 Schematische Darstellung des Informationsflusses zur Altschäle . . . . . 20

3.1 Mittlere Höhe der Inventurpunkte . . . . . . . . . . . . . . . . . . . 36

3.2 Mittlere Hangneigung der Inventurpunkte . . . . . . . . . . . . . . . 36

3.3 Anteile der Hangklassen an den Datensets . . . . . . . . . . . . . . . . 37

3.4 Exposition homogener Plots im Harz . . . . . . . . . . . . . . . . . 39

3.5 Exposition homogener Plots im Solling . . . . . . . . . . . . . . . . . . 39

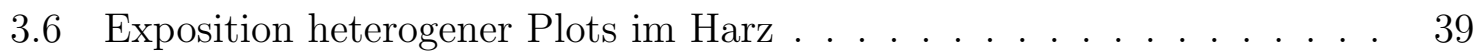

3.7 Exposition heterogener Plots im Solling . . . . . . . . . . . . . . . . . . 39

3.8 Exposition heterogener Plots gruppiert in Steigungsklassen . . . . . . . 40

3.9 Exposition homogener Plots gruppiert in Steigungsklassen . . . . . . . 41

3.10 Exponiertheit homogener und heterogener Plots im Solling . . . . . . . 42

3.11 Exponiertheit homogener und heterogener Plots im Harz . . . . . . . . 42

3.12 Potentieller Strahlungsinput homogener und heterogener Plots im Solling 43

3.13 Potentieller Strahlungsinput homogener und heterogener Plots im Harz 43

3.14 Verteilung des Bestandesalters der Probeflächen im Harz . . . . . . . . 44

3.15 Verteilung des Bestandesalters der Probeflächen im Solling . . . . . . . 44

3.16 Anteile der Bestandestypengruppen an Fichtenplots . . . . . . . . . . . 46

3.17 Verteilung der Inventurpunkte in Klassen unterschiedlicher Baumartenzahl je Abteilung im Solling . . . . . . . . . . . . . . . . . . . . . . 46

3.18 Verteilung der Inventurpunkte in Klassen unterschiedlicher Baumartenzahl je Abteilung im Harz . . . . . . . . . . . . . . . . . . 46 
3.19 Verteilung der Inventurpunkte in Klassen unterschiedlicher Baumartenzahl je Unterfläche im Solling . . . . . . . . . . . . . . . . . . . . . 47

3.20 Verteilung der Inventurpunkte in Klassen unterschiedlicher Baumartenzahl je Unterfläche im Harz . . . . . . . . . . . . . . . . . . . . . 47

3.21 Kumulierte Prozente der Baumartenzahl je Unterfläche im Harz . . . . 48

3.22 Kumulierte Prozente der Baumartenzahl je Unterfläche im Solling . . . 48

3.23 Kumulierte Prozente der Baumartenzahl je Abteilung im Harz . . . . . 48

3.24 Kumulierte Prozente der Baumartenzahl je Abteilung im Solling . . . . 48

3.25 Jagdstreckenaufkommen des Rotwildes . . . . . . . . . . . . . . . 49

3.26 Relativer Variationskoeffizient der Monatsmittelwerte der Rotwildstrecke 50

3.27 Anteil der Hauptjagdperiode an der Gesamtjahresstrecke . . . . . . . . 50

3.28 Relativer Anteil der Reviere an Streckenklassen im Harz . . . . . . . . 52

3.29 Relativer Anteil der Reviere an Streckenklassen im Solling . . . . . . . 52

3.30 Relativer Anteil der Abteilungen mit Erlegungen . . . . . . . . . . . . . 53

3.31 Mittlere Anzahl der Erlegungen je Abteilung . . . . . . . . . . . . . . . 53

4.1 Häufigkeit von Winterneuschäle, klassifiziert für Solling und Harz . . . 56

4.2 Mittlere Winterneuschälprozente in Solling und Harz, getrennt nach Inventurjahren . . . . . . . . . . . . . . . . 56

$4.3 \quad$ Mittleres Neuschälprozent der Inventurpunkte je Höhenklasse im Harz . $\quad 62$

4.4 Mittleres Neuschälprozent der Inventurpunkte für niedrige, mittlere und hohe Lagen im Harz . . . . . . . . . . . . . . . . . . . . 62

4.5 Mittlere Neuschälprozente nach Hangneigung getrennt für beide Untersuchungsgebiete mit dem Median als Klassengrenze . . . . . . . . . . . 67

5.1 Relative Abweichung der mittleren Schälprozente einzelner Klassen des

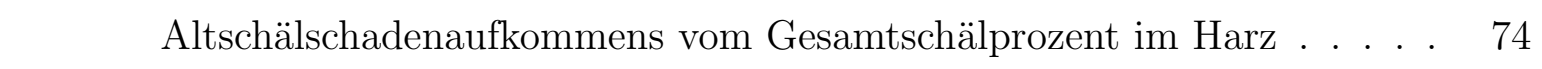

$5.2 \quad$ Relative Abweichung der mittleren Schälprozente einzelner Klassen des Altschälschadenaufkommens vom Gesamtschälprozent im Solling . . . . 74

5.3 Relative Abweichung der mittleren Schälprozente einzelner Kontinuitätsklasser vom Gesamtschälprozent im Harz . . . . . . . . . . . . . . . . . . . 74

5.4 Relative Abweichung der mittleren Schälprozente einzelner Kontinuitätsklassen vom Gesamtschälprozent im Solling . . . . . . . . . . . . . . . . . . 74

5.5 Relative Abweichung der mittleren Schälprozente einzelner Klassen der Höhenlage ü.NN vom Gesamtschälprozent im Harz . . . . . . . . . . . 75 
$5.6 \quad$ Relative Abweichung der mittleren Schälprozente einzelner Klassen der

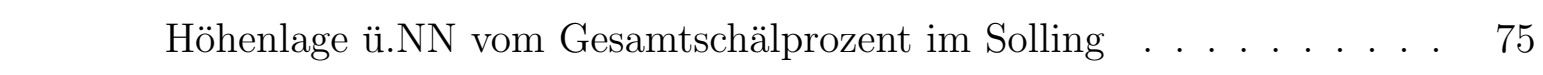

5.7 Relative Abweichung der mittleren Schälprozente einzelner Hangnei-

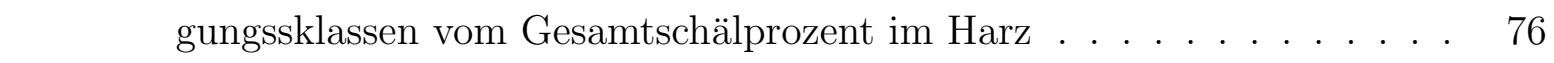

5.8 Relative Abweichung der mittleren Schälprozente einzelner Hangnei-

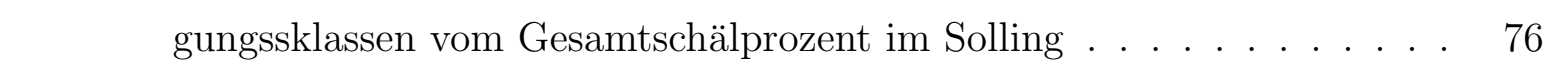

5.9 Relative Abweichung der mittleren Schälprozente einzelner Expositionsklassen vom Gesamtschälprozent im Harz . . . . . . . . . . . . . . . . . 77

5.10 Relative Abweichung der mittleren Schälprozente einzelner Expositionsklassen vom Gesamtschälprozent im Solling . . . . . . . . . . . . . . . . 77

5.11 Relative Abweichung der mittleren Schälprozente einzelner Exponiert-

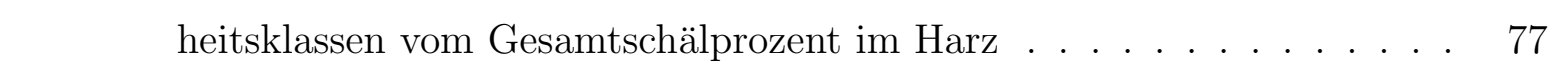

5.12 Relative Abweichung der mittleren Schälprozente einzelner Exponiert-

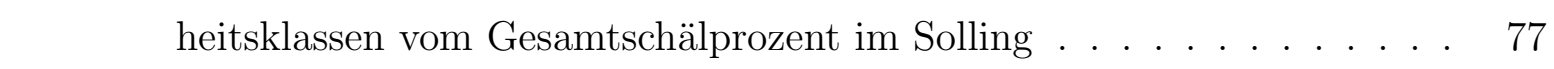

5.13 Relative Abweichung der mittleren Schälprozente einzelner Strahlungs-

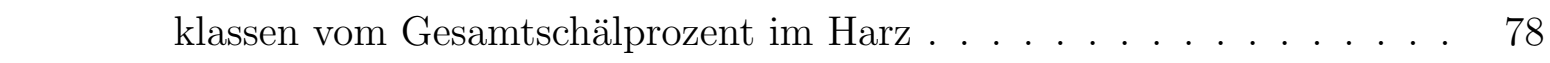

5.14 Relative Abweichung der mittleren Schälprozente einzelner Strahlungs-

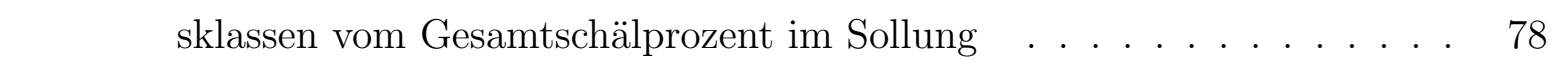

5.15 Relative Abweichung der mittleren Schälprozente einzelner Klassen des

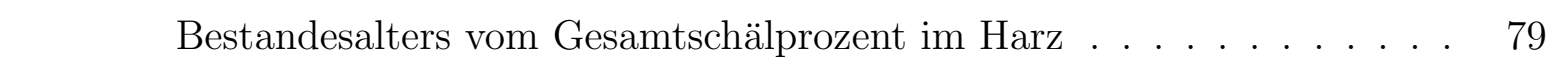

5.16 Relative Abweichung der mittleren Schälprozente einzelner Klassen des

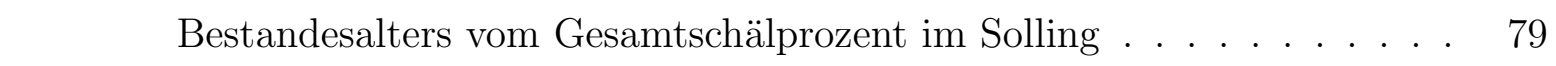

5.17 Relative Abweichung der mittleren Schälprozente einzelner Klassen der

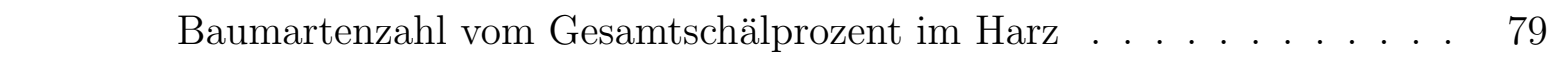

5.18 Relative Abweichung der mittleren Schälprozente einzelner Klassen der

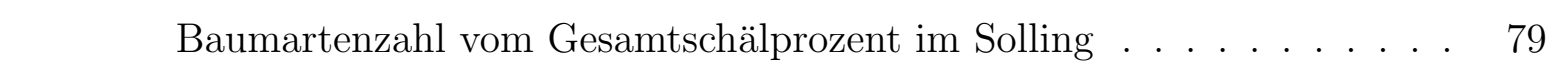

5.19 Relative Abweichung der mittleren Schälprozente einzelner Klassen des Jagdstreckenindizes vom Gesamtschälprozent im Harz . . . . . . . . . . 80

5.20 Relative Abweichung der mittleren Schälprozente einzelner Klassen des

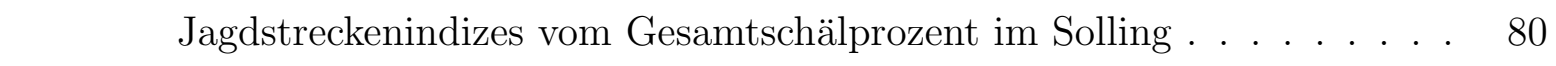

5.21 Relative Abweichung der mittleren Schälprozente einzelner Wegeklassen vom Gesamtschälprozent im Harz . . . . . . . . . . . . . . . . . . . . 81 



\section{Tabellenverzeichnis}

$2.1 \quad$ Flächengrößen der einzelnen Bezugsebenen . . . . . . . . . . . . . . . . 13

2.2 Stichprobenumfang der Inventuren zur Schälschädigung . . . . . . . . . 16

2.3 Anzahl reiner Fichten- oder Buchenstichprobenpunkte. . . . . . . . . . 17

2.4 Gesamtübersicht zum jeweils verwendeten Datenpool in den Kapiteln . 19

2.5 Grenzwerte der Expositionsklassen . . . . . . . . . . . . . . . . . . . . 23

3.1 Mittlere Höhe homogener und heterogener Sets in Solling und Harz . . 36

3.2 Mittlere Hangneigung homogener und heterogener Sets in Solling und Harz . . . . . . . . . . . . . . . . . . 37

3.3 Mittlere relative Anteile ausgewählter Monate am Gesamtaufkommen der Rotwildjagdstrecken in Harz und Solling . . . . . . . . . . . . . . . 50

3.4 Jährliches mittleres Rotwildstreckenaufkommen in Stück je 100 ha im Zeitraum September bis Januar . . . . . . . . . . . . . . . . 52

$4.1 \quad$ Mittleres Winterneuschälprozent an Fichte in Harz und Solling der Jahre $2002,2003,2004$ und 2006 . . . . . . . . . . . . . . . . 56

4.2 Mittleres Winterneuschälprozent an Fichte in Harz und Solling der Jahre 2002, 2003, 2004 und 2006, unterschieden zwischen homogen und heterogen besetzten Plots . . . . . . . . . . . . . . . . . 57

4.3 Mittleres Winterneuschälprozent an Fichte in Harz und Solling im Jahr 2006, bezogen auf Klassen vorangegangenen Neuschälvorkommens . . . 58

4.4 Mittleres Winterneuschälprozent an Fichte in Harz und Solling im Jahr 2006, bezogen auf Klassen dokumentierter Altschälschäden . . . . . . . 60

4.5 Mittleres Neuschälprozent an Fichte in Harz und Solling, bezogen auf Klassen der Höhenlage ü.NN] . . . . . . . . . . . . . . . . . . . . . 61

$4.6 \quad$ Mittleres Neuschälprozent an Fichte im Harz, bezogen auf Klassen der

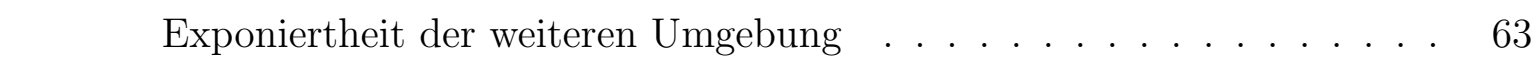


$4.7 \quad$ Mittleres Neuschälprozent an Fichte im Solling, bezogen auf Klassen der Exponiertheit der weiteren Umgebung . . . . . . . . . . . . . . 64

4.8 Mittleres Winterneuschälprozent an Fichte in Harz und Solling 20022006, bezogen auf Hangneigungsklassen . . . . . . . . . . . . . . . 65

4.9 Mittleres Winterneuschälprozent an Fichte in Harz und Solling 20022006, bezogen auf Hangneigungsklassen; Median als Klassengrenze . . . 66

4.10 Mittleres Winterneuschälprozent an Fichte in Harz und Solling 20022006, bezogen auf Expositionsklassen . . . . . . . . . . . . . . . 68

4.11 Mittleres Winterneuschälprozent an Fichte in Harz und Solling 20022006, bezogen auf Strahlungsklassen . . . . . . . . . . . . . . . . . . 69

4.12 Mittlerer Jagdstreckenindex der Hauptbejagungszeit und mittleres Winterneuschälprozent an Fichte je Forstamt in Harz und Solling 2002-2006 69

4.13 Gegenüberstellung des jährliches mittleren Rotwildstreckenaufkommens im Zeitraum September bis Januar mit dem jeweiligen Neuschälprozent der Periode . . . . . . . . . . . . . . . . . . . . . 70

4.14 Mittleres Winterneuschälprozent an Fichte in Harz 2002-2006, bezogen auf Nähe zu Wanderwegen . . . . . . . . . . . . . . . . . . . . . 71

$6.1 \quad$ Modellanpassungen und Klassifizierungen der Harzinventuren. . . . . . 83

6.2 Erklärende Variablen der logistischen Regression Harz 2002 . . . . . . . 85

6.3 Erklärende Variablen der logistischen Regression Harz 2004 . . . . . . . 86

$6.4 \quad$ Erklärende Variablen der logistischen Regression Harz 2006 . . . . . . . 87

6.5 Modellanpassungen und Klassifizierungen der Sollinginventuren . . . . 88

$6.6 \quad$ Erklärende Variablen der logistischen Regression Solling 2002 . . . . . . 89

6.7 Erklärende Variablen der logistischen Regression Solling 2003 . . . . . . 90

6.8 Erklärende Variablen der logistischen Regression Solling 2006 . . . . . . 90

6.9 Variablen im Gesamtmodell für Harz und Solling . . . . . . . . . . . . 92

$7.1 \quad$ GLM Harz und Solling 2002-06 … . . . . . . . . . . . . . . . . . . . 95

7.2 GLM Harz und Solling . . . . . . . . . . . . . . . . . . . . . . . 96

7.3 Modellvariablen nach der Methode stepAIC für erste U Harz und Solling 98

7.4 GLM Harz . . . . . . . . . . . . . . . . . . . . . . . . . . . . . . . . . . . . . . . . . 99

7.5 GLM Solling . . . . . . . . . . . . . . . . . . . . . . . . . 100

$7.6 \quad$ Modellvariablen nach der Methode stepAIC für Harz und Solling. . . . . 102

7.7 GLM Geodaten Harz und Solling . . . . . . . . . . . . . . . . . . . . . 104 
$7.8 \quad$ GLM Rest Harz und Solling . . . . . . . . . . . . . . . . . . . . 104

7.9 Modellvariablen nach der Methode stepAIC für Gruppen Harz und Solling 105

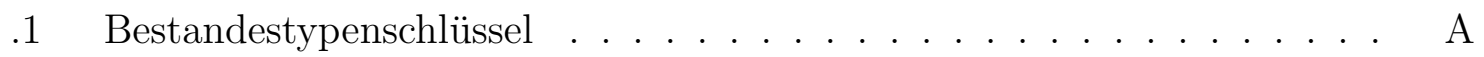

.2 Baumartenschlüssel . . . . . . . . . . . . . . . . . . . . . . B 


\section{Literaturverzeichnis}

Adrados, C., Baltzinger, C., Janeau, G. \& Pépin, D. (2008): Red deer Cervus elaphus resting place characteristics obtained from differential GPS data in a forest habitat. European Journal of Wildlife Research (54), 487-494

Ahlen, I. (1965): A comparison between the winter feeding behaviour of the red deer and the moose. In:Proceeding of the 6th Congress of the International Union of Game Biologists (Ed. by T.H. Blank), S. 117-126. The Nature Conservancy, Bournemouth

Akashi, N. \& Nakashizuka, T. (1999): Effects of bark-stripping by Sika deer (Cervus nippon) on population dynamics of a mixed forest in Japan.. Forest Ecology and Management 113, 75-82

AKS (1980): Arbeitskreis Forstliche Standortskartierung. Forstliche Standortsaufnahme. 4. Aufl., Münster

Albers, U., Hullen, M., Böckmann, T. \& Hooge, H. (2004): Waldentwicklung im Nationalpark Harz. Eine Bilanz 10 Jahre nach der Einrichtung des Nationalparks Harz, unveröffentlicht

Altman, M. (1958): The flight distance in free-ranging big game. Journal of Wildlife Management 22, 207-209

Amler, K., Bahl, A., Henle, K., Kaule, G., Poschlod, P. \& Settele, J. (Hrsg.) (1999): Populationsbiologie in der Naturschutzpraxis - Isolation, Flächenbedarf und Biotopansprüche von Pflanzen und Tieren. Verlag Ulmer, 336 Seiten

Ammer, C. (1996): Impact of ungulates on structure and dynamics of natural regeneration of mixed mountain forests in the Bavarian Alps. Ungulates in Temperate Forest Ecosystems. Orest Ecology and Management (88), 43-53 
Andersen, J. (1953): Analysis of the Danish roe deer population based on the extermination of the total stock. Danish Rev. Game Biol. 2, 127-155

Ando, M., Itaya, A., Yamamoto, S. I. \& Shibata, E. (2006): Expansion of dwarf bamboo, Sasa nipponica, grassland under feeding pressure of sika deer, Cervus nippon, on subalpine coniferous forest in central Japan. Journal of Forest Research 11, 51-55

Ando, M., Yokota, H.-O. \& Shibata, E. (2003): Bark stripping preference of Sika deer, Cervus nippon, in termsof bark chemical contents. Forest Ecology and Management 177, 323-331

Arnold, W., Ruf, T., Reimoser, S., Tataruch, F., Onderscheka, K. \& Schober, F. (2004): Nocturnal hypometabolism as an overwintering strategy of red deer (Cervus elaphus). Am J Physiol Regul Integr Comp Physiol 286(1), 174-181

Baderschneider, A. \& Böckmann, T. (2004): Holzaufkommen im Niedersächsischen Teil des Harzes. http://www.landesforsten.de, unveröffentlicht

Begon, M., Harper, J. L. \& Townsend, C. R. (1991): Ökologie - Individuen, Populationen, Lebensgemeinschaften. Birkhäuser Verlag. Basel, Boston, Berlin, 1024 Seiten

Behrend, D. F. \& Lubeck, R. A. (1968): Summer flight behavior of white-tailed deer in two Adirondack forests. Journal of Wildlife Management 32(3), 615-618

Beisel, R. (1973): Auswirkungen von Beschädigungen der unteren Stammabschnitte auf den Wert und die Produktionsdauer von Fichtenbetriebsklassen und von Fichtenbeständen. Mitteilungen der Forstlichen Versuchs- und Forschungsanstalt Baden-Württemberg, Heft 48

Bencze, L. (1977): Umfang von Schälschäden des Rotwildes (Cervus elaphus L.) und Holzwertminderung in Pappelbeständen. Zeitschrift für Jagdwissenschaft 23, 214-218

Binder, B. (1991): Hilfstafeln zur Erhebung und Bewertung von Schälschäden an Fichte. Forstliche Bundesversuchsanstalt Wien. Eigenverlag, Wien

Bonenfant, C., Gaillard, J. M., Klein, F. \& Loison, A. (2002): Sex- and age-dependent effects of population density on life history traits of red deer Cervus elaphus in a temperate forest. Ecography (25), 446-458 
Borchers, K. (1964): Die Eis- und Schneebruchlagen des Harzes. Aus dem Walde, 8

Borkowski, J. (2001): Flight behaviour and observability in human-disturbed sika deer. Acta Theriologica 46, 195-206

Borkowski, J. \& Ukalska, J. (2008): Winter habitat use by red and roe deer in pine-dominated forest. Forest Ecology and Management (3), 468-475

Borkowski, J. \& Ukalski, K. (2012): Bark stripping by red deer in a post-disturbance area: The importance of security cover. Forest Ecology and Management 263(1), $17-23$

Borowski, S. \& Kossak, S. (1975): The food habits of deer in the Bialowieza Primeval Forest. Acta Theriologica 20, 463-506

Boyce, M. S., Mao, J. S., Merrill, E. H., Fortin, D., Turner, M. G., Fryxell, J. \& Turchin, P. (2003): Scale and heterogeneity in habitat selection by elk in Yellowstone National Park. Ecoscience 10(4), 421-431

Boyle, S. A. \& Samson, F. B. (1985): Effects of non-consumptive recreation on wildlife: a review. Wildl Soc Bull 13, 110-116

Brownie, C., Hines, J. E., Nichols, J. D., Pollock, K. H. \& Hestbeck, J. B. (1993): Capture-recapture studies for multiple strata including non-Markovian transitions. Biometrics 49, 1173-1187

Bubenik, A. \& Lochmann, J. (1956): Futterverbrauch und Tagesrhythmus der Futteraufnahme bei Reh- und Rotwild. Zeitschrift für Jagdwissenschaft 2, $112-118$

Bubenik, A. B. (1965): Beitrag zur Geburtskunde und zu den Mutter-Kind-Beziehungen des Reh- (Capreolus capreolus L.) und Rotwildes (Cervus elaphus L.). Zeitschrift für Säugetierkunde 30, 65-128

Bücking, M., Moshammer, R., Schmitz, W., Jochum, M. \& Roeder, A. (2006): Hilfstabellen zur einfachen Bewertung von Schälschäden in den Wäldern von Rheinland-Pfalz. Forst und Holz, 61 (5)

Bugalho, M. N. \& Milne, J.A. (2003): The composition of the diet of red deer (Cervus elaphus) in a Mediterraneanenvironment: a case of summer nutritional constraint?. Forest Ecology and Management 181, 23-29 
Burrough, P. A. \& McDonnel, R. A. (1998): Principles of geographical information systems. Spatial information systems and geostatistics. Oxford

Burschel, P. (1975): Schalenwildbestände und Leistungsfähigkeit des Waldes als Problem der Forst- und Holzwirtschaft aus der Sicht des Waldbaus. Allgemeine Forstzeitschrift, 30

Burschel, P. (1993): Wildschadensvorbeugung. Wald, 43

Burschel, P. \& Huss, J. (1987): Grundriß des Waldbaus. Verlag Paul Parey, Hamburg

Bützler, W. (2001): Rotwild: Biologie, Verhalten, Umwelt, Hege. 5., überarbeitete u. erweiterte Auflage. BLV Verlag, München, Wien, Zürich, 264 Seiten

Clutton-Brock, T. H. (1988): Reproductive success: studies of individual variation in contrasting breeding systems. University of Chicago Press, Chicago

Clutton-Brock, T. H., Guinness, F. E. \& Albon, S. D. (1982): Red deer: behavior and ecology of two sexes. University of Chicago Press, Chicago

Danell, K., Bergström, R., Edenius, L. \& Ericsson, G. (2003): Ungulates as drivers of tree population dynamics at module and genet levels. Forest Ecology and Management (181), 67-76

Danell, K., Niemelä, P., Varvikko, T. \& Vuorisalo, T. (1991): Moose browsing on Scots pine along a gradient of plant productivity. Ecology 72, 1624-1633.

Dauster, K. H. (1939): Untersuchungen über die Zusammensetzung und Aufnahme der Äsung des Rotwildes und ihre Beziehung zur Schälfrage. Mitt. Forstw. Forstwiss, 216-230

De Crombrugghe, S. A. (1965): Beziehung zwischen dem Umfang der Rotwildschäle in Buchenbeständen und den Niederschlägen. Zeitschrift für Jagdwissenschaft $11,184-191$

Debeljak, M., Dzeroski, S., Jerina, K., Kobler, A. \& Adamic, M. (2001): Habitat suitability modelling for red deer (Cervus elaphus L.) in South-central Slovenia with classification trees. Ecological Modelling (138), 321-330

Dzieciolowski, R. (1967): Food of the red deer in an annual cycle. Acta Theriologica $12,503-520$ 
Dzieciolowski, R. (1969): The quantity, quality and seasonal variation of food resources available to red deer in various environmental conditions of forest Management. Forest Research Institute Warschau

Faber, W. E. (1996): Bark stripping by moose on young Pinus sylvestris. South Central Sweden. Scandinavian Journal of Forest Research 11, 300-306

Gebert, C. \& Verheyden-Tixier, H. (2001): Variations of diet composition of red deer (Cervus elaphus L.) inEurope. Mammal Review 31, 189-201

Gerke, R. (1980): Bewertung von Schälschäden - dargestellt am Beispiel der Fichte Großräumige Erhebung betriebswirtschaftlicher und waldbaulicher Auswirkungen. Dissertation. Albert-Ludwigs-Universität Freiburg i.B., 123 Seiten

Gill, R. M. A. (1990): Monitoring the status of European and North American Cervids. Nairobi, Kenya

Gill, R. M. A. (1992): A Review of Damage by Mammals in North Temperate Forests: 1. Deer. Forestry 65(2), 145-169

Gill, R. M. A. (2000): The impact of deer on woodland biodiversity. Forestry Commission Information Note 36

Gill, R. M. A. (2006): The influence of large herbivores on tree recruitment and forest dynamics. In: Danell K., Bergstrom, R. and Rooke T. (eds). Large Herbivore Ecology, Ecosystem Dynamics and Conservation. Cambridge University Press, Cambridge, UK: 170-202

Gill, R. M. A. \& Beardall, V. (2001): The impact of deer on woodlands: the effects of browsing and seed dispersal on vegetation structure and composition. Forestry 74, 209-218

Gordon, H., Hester, A. J. \& Festa-Bianchet, M. (2004): The management of wild large herbivores to meet economic, conservation and environmental objectives. Journal of Applied Ecology 41, 1021-1031

Gossow, H. (1988): Fütterungsstandort und Rotwildschäle. Österreichische Forstzeitung 6, 53-54 
Groot Bruinderink, G. W. T. A. \& Hazebroek, E. (1995): Ingestion and diet composition of red deer (Cervuselaphus L.) in the Netherlands. Mammalia 59, 187-195

Hediger, H. (1946): Bemerkungen zum Raum-Zeit-System der Tiere. Schweiz. Z. Psychologie 5, 241-269

Heinze, E., Boch,S., Fischer, M., Hessenmöller, D., Klenk, B.,Müller, J., Prati, D., Schulze, e.-D., Seele, C., Socher, S. \& Halle, S. (2011): Habitat use of large ungulates in northeastern Germany in relation to forest management. Forest Ecology and Management 261(2), 288-296

Hofmann, R. R. (1989): Evolutionary steps of ecophysiological adaptation and diversification of ruminants: acomparative view of their digestive system. Oecologia 78, 443-457

Hofmann, R. R. \& Stewart, D. R. M. (1972): Grazers and browsers: a classification based on the stomach structure and feeding habits of East African ruminants. Mammalia 36, 226-240

Hörnberg, S. (2001): Changes in population density of moose (Alces alces) and damage to forests in Sweden. Forest Ecology and Management 149, 141-151

Hosmer, D. W. \& Lemeshow, S. (2000): Applied Logistic Regression. 2. Auflage, John Wiley and Sons, New York

Hoste, H., Jackson, F., Athanasiadou, S., Thamsborg, S. M. \& Hoskin, S. O. (2006): The effects of tannin-rich plants on parasitic nematodes in ruminants. Trends in Parasitology 22, 253-261

Jalkanen, A. (2001): The probability of moose damage at the stand level in southern Finland. Silva Fennica 35(2), 159-168

Jamrozy, G. (1980): Winter food resources and food preferences of red deer in Carpathian Forest. Acta Theriologica 25, 221-238

Jensen, P. V. (1968): Food selection of the Danish red deer (Cervus elaphus) as determined by examination ofthe rumen content. Danish Review of Game Biology 5, 1-44

Jeppesen, J. L. (1987): Impact of human disturbance on home range, movements and activity of red deer in a Danish environment. Dan Rev Game Biol 13, 1-33 
Jerina, K. (2006): Spatial distribution, home range and body mass of red deer (Cervus elaphus L.) in regard to environmental factors. Dissertation. Universität von Ljubljani, Biotechnische Fakultät. 172 Seiten

Kaji, K., Ohtaishi, N. \& Koizvmi, T. (1984): Population growth and its effect upon the forest used by sika deer on Nakanoshima Island in Laka Toya, Hokkaido. Acta Zoologica Fennica 172, 203-205

Kato, F. (1969): Stammfäuleschäden der Fichte. Ein Beitrag zur Schadensrechnung in den staatlichen Fichtenwäldern Niedersachsens mit besonderer Berücksichtigung der Schälschäden des Rotwildes. Forstarchiv 40, 81-92

Kato, F. (1981): Ein einfaches Verfahren zur Schälschadensbewertung für Entschädigungszwecke bei der Fichte. Der Forst- und Holzwirt, 36. Verlag M. \& H. Schaper, Hannover

Kato, F. (1983): Probleme der Schälschadensbewertung für Entschädigung und Lösungsmöglichkeiten bei der Fichte. Forstarchiv, 54. Verlag M. \& H. Schaper, Hannover

Kiffner, C., Rössiger, E., Trisl, O., Schulz, R. \& Rühe, F. (2008): Probability of recent bark stripping damage by Red Deer (Cervus elaphus) on Norway Spruce (Picea abies) in a low mountain range in Germany. Silva Fennica 42(1), 125-134

Kim, Y. S. (1983): Pathologische Veränderungen an Fagus sylvatica L. nach mechanischen Verletzungen. Wien

Klemen, J., Mihec, D. \& Miha, A. (2008): Red deer (Cervus elaphus) bark stripping on spruce with regard to spatial distribution of supplemental feeding places. Zbornik gozdarstva in lesarstva 86, 33-43

Knigge, W. (1975): Die Auswirkungen von Schälschäden auf die Rohholzeigenschaften von Fichte und Buche. Forstarchiv 46, 32-38

König, E. (1969): Schälschadenverhütung durch geeignete Rotwilfütterung. Der Deutsche Jäger 87, 479-483

Kraft, G. (1884): Beiträge zur Lehre von den Durchforstungen, Schlagstellungen und Lichtungshieben. Klindworth, Hannover

Kramer, H. \& Womelsdorf, G. (1985): Vermehrter Schneebruch durch Rotwildschälschäden bei Fichte. Der Forst- und Holzwirt 
Kraus, P. (1984): Ergebnisse pflanzensoziologischer Aufnahmen als möglicher Indikator der relativen Rotwilddichte unter Berücksichtigung von Wildschäden und Wildbretgewichten in ausgewählten Revieren der Eifel. FHS Hildesheim, Holzminden, Göttingen

Kraus, P. (1987): Vegetationsbeeinflussung als Indikator der relativen Rotwilddichte. Zeitschrift für Jagdwissenschaft 33, 42-59

Kroth, W., Sinner, H.-U. \& Bartelheimer, P. (1984): Hilfsmittel zur Bewertung von Schälschäden an Fichte und Buche. Allgemeine Forstzeitschrift, 39

Kuiters, A. T. \& Slim, P. A. (2002): Regeneration of mixed deciduous forest in a Dutch forest-heathland, following a reduction of ungulate densities. Biological Conservation 105, 65-74

Kuiters, A. T., Mohren, G. M. J. \& Van Wieren, S. E. (1996): Ungulates in temperate forest ecosystems. Forest Ecology and Management 88, 1-5

Kuiters, A. T., van der Sluijs, L. A. M. \& Wytema, G. A. (2006): Selective bark-stripping of beech, Fagus sylvatica, by free-ranging horses. Forest Ecology and Management 222, 1-8

Latham, J., Staines, B. W. \& Gorman, M.L. (1999): Comparative feeding ecology of red (Cervus elaphus) androe deer (Capreolus capreolus) in Scottish plantation forests. Journal of Zoology 247, 409-418

Licoppe, A. M. (2006): The diurnal habitat used by red deer (Cervus elaphus L.) in the Haute Ardenne. European Journal of Wildlife Research 52(3), 164-170

Litz, H. P. (2000): Multivariate statistische Methoden und ihre Anwendung in den Wirtschafts- und Sozialwissenschaften. R. Oldenbourg, München-Wien

Löffler, H. (1975): Zur Ausbreitung der Wundfäule in der Fichte.

Forstwissenschaftliches Centralblatt. 94. Verlag Paul Parey, Hamburg und Berlin

Loison, A., Toigo, C. \& Gaillard, J.M. (2003): Large herbivore biodiversity in European mountain ecosystems: Current status and challenges for the future. In: Nagy, L. (ed.) Alpine Biodiversity - Patterns, Process and Change in Europe, 351-366

Loudon, A. S. I. (1985): Lactation and neonatal survival of mammals. Symposium of the Zoological Society of London 54, 183-207 
Maizeret, C. \& Ballon, P. (1990): Analysis of causal factors behind cervid damage on the cluster pine in the landes of Gascony. Gibier Faune Sauvage (7), 275-291

Mark A. White (2012): Long-term effects of deer browsing: Composition, structure and productivity in a northeastern Minnesota old-growth forest. Forest Ecology and Management 269

Marwede, M.-C. \& Schneider, M. O. (2004): Räumliche Verteilung geschälter Bäume in Beziehung zum Wegenetz und zu Jagdeinrichtungen im Solling. Göttingen

Matschullat, J., Heinrichs, H., Schneider, J. \& Ulrichs, B. (1994): Gefahr für Ökosysteme und Wasserqualität. Ergebnisse interdisziplinärer Forschung im Harz Springer, Berlin, Heidelberg, New York

Mayer, H. (1973): Möglichkeiten und Grenzen der Schalenwildhege im Gebirgswald. Wald und Wild, Beitrag zum Schweizer Forstverein 52. Zürich

Mayer, H. (1975): Schäden durch überhöhte Wildbestände - ein volkswirtschaftliches Problem. Allgemeine Forst- und Jagdzeitung, 30

Mclntyre, E. B. (1975): Bark stripping by ungulates. PhD Thesis, University of PhD Thesis, University of Edinburgh

Milner, J. M., Bonenfant, C., Mysterud, A., Gaillard, J. M., Csányi, S. \& Stenseth, N. C. (2006): Temporal and spatial development of red deer harvesting in Europe: biological and cultural factors. Journal of Applied Ecology (43), 721-734

Min, B. R., Barry, T. N., Attwood, G. T. \& McNabb, W. C. (2003): The effect of condensed tannins on the nutrition and health of ruminants fed fresh temperate forages: a review. Animal Feed Science and Technology 106, 3-19

Mitchell, B. \& McCowan, D. (1986): Patterns of damage in relation to the site preferences of deer in an enclosedplantation of sitka spruce and Lodgepole pine. Scottish Forestry 40, 107-117

Møller, A. P., Dufva, R. \& Allander, K. (1993): Parasites and the evolution of host social behavior. Advances in the Study of Behavior 22, 65-102

Moog, M. (2008): Bewertung von Wildschäden im Wald. Verlag J.

Neumann-Neudamm AG, Melsungen 
Motta, R. (1997): Dendroecology in ungulate forest damage. Dendrochronologia 15, $11-20$

Mues, V. (2000): GIS-gestützte Regionalisierung von Klima- und Depositionsdaten in Niedersachsen. Dissertation. Georg-August-Universität Göttingen

Müller, E. (1979): Vom Schälen des Rotwildes. Der Anblick, 45-46

Müller, G. (1985): Beiträge zur Erfassung und Ursachenanalyse von Rotwildschälung im Nordschwarzwald. Dissertation. Albert-Ludwigs-Universität Freiburg i.B., 140 Seiten

Müller, H.-J. (1963): Untersuchungen zur Bemessung der wirtschaftlich tragbaren Wilddichte im Walde nach Standort und Wildschaden. Forstliche Fakultät Eberswalde, Dissertation

Mulvey, M., Aho, J. M. \& Rhodes Jr, O. E. (1994): Parasitism and white-tailed deer: timing and components of female reproduction. Oikos 70, 177-182

Mysterud, A., Yoccoz, N. G., Langvatn, R., Pettorelli, N. \& Stenseth, N. C. (2007): Hierarchical path analysis of deer responses to direct and indirect effects of climate in northern forest. Phil. Trans. R. Soc. B, 1-10

Opitz, S., Köhler, G. \& Mabelis, A. (1998): Local movement of the grasshopper Chorthippus parallelus (Zett.). Proc. Exper. \& Appl. Entomology 9, 53-58.

Otto, L.-F. (1985): Der Einfluss morphologischer Baummerkmale auf die Schneebruchstabilität ausgewählter Fichtenjungbestände des Thüringer Waldes. Diplomarbeit an der TU Dresden, Sektion Forstwirtschaft

Persson, I.-L., Danell, K. \& Bergstrøm, R. (2000): Disturbance by large herbivores in boreal forests with special reference to moose. Ann. Zool. Fenn. 37, 251-263

Petrak, M. (1982): Etho-ökologische Untersuchungen an einer Rothirschpopulation (Cervus elaphus Linné, 1758) der Eifel unter besonderer Berücksichtigung des stoffwechselbedingten Verhaltens. Schriftenreihe Arbeitskreis Wildbiologie an der Justus-Liebig-Universität Gießen, 196 S.

Pheiffer, J. \& Hartfield, B. (1984): Beziehungen zwischen der Winterfütterung und dem Schälverhalten des Rotwildes in der Eifel. Zeitschrift fûr Jagdwissenschaft $30,243-255$ 
Picard, J. F., Oleffe, P. \& Boisaubert, B. (1991): Influence of oak mast on feeding behaviour of red deer (Cervus elaphus). Annales des Sciences Forestières 48, $547-559$

Prien, S. (1997): Wildschäden im Wald. Parey Buchverlag, Berlin

Primack, R. B. (1995): Naturschutzbiologie. Spektrum Akademischer Verlag. Heidelberg, Berlin, Oxford, 713 Seiten

R Development Core Team (2006): R: A language and environment for statistical computing. Vienna, Austria: R Foundation for Statistical Computing

Rademacher, P. (2005): Contents of nutrient elements in tree components of economical important species inrelation to their residual utilisation. Holz als Roh- und Werkstoff 63, 285-296

Randveer, T. \& Heikkilä, R. (1996): Damage caused by moose (Alces alces) by bark stripping of Picea abies. Scandinavian Journal of Forest Research 11(2), 153-158

Richter, J. (1974): Die Ausbreitung der Rotfäule in der Fichte. Allgemeine Forstzeitschrift, 29. BLV Verlagsgesellschaft, München

Roeder, A. (1970): Schälschaden des Rotwildes (Cervus elaphus L.) an der Fichte (Picea abies (L.) Karst). Dissertation. Georg-August-Universität Göttingen, 146 Seiten

Rudolf, M. \& Müller, J. (2004): Multivariate Verfahren - Eine praxisorientierte Einführung mit Anwendungsbeispielen in SPSS. Hogrefe. Göttingen. 331 Seiten

Saint-Andrieux, C. \& Klein, F. (1995): Sensibilité des différentes essences forestières aux dégâts d'ongulés surle massif vosgien alsacien. Bulletin Mensuel de l'ONC $196,2-12$

Schaller, M. (2002): Ökonomische Bewertung von Schälschaden im Wald. München

Schmidt, K. (1993): Winter ecology of nonmigratory Alpine red deer. Oecologia 95, 226-233

Schönhar, S. (1975): Untersuchungen über den Befall rückegeschädigter Fichten durch Wundfäulepilze. AFJZ 146, 72-75

Schultz, R. D. \& Bailey, J. A. (1978): Responses of National Park Elk to Human Activity. Journal of Wildlife Management 42, 91-100 
Schulz, R. (2003): Flächenbezogene Modelle zur Unterstützung der Forstlichen Standortskartierung im Niedersächsischen Bergland. Dissertation, Georg-August-Universität, Göttingen, 340 Seiten

Schwietert, B. (1989): Geologie, Klima und Forststandorte des Harzes. Ergebnisse neuer Standortskartierungen, AFZ (18-20), 449-453

Senn, O. (1987): Végétaux consommés par les cerfs en période de chasse dans la région du Beauchêne (Hautes-Alpes). Bulletin Mensuel de l'ONC 114, 31-34

Settele, J. (1998): Metapopulationsanalyse auf Rasterdatenbasis - Möglichkeiten des Modelleinsatzes und der Ergebnisumsetzung im Landschaftsmaßstab am Beispiel von Tagfaltern. Teubner Verlag. Stuttgart \& Leipzig

Skovlin, J. M. (1982): Habitat requirements and evaluations. In: Thomas, J. W. and Toweill, D.E. (Hrsg.). Elk of North America - ecology and management. Wildlife Management Institute, Harrisburg. S. 219-277

Smart, J. C. R., Ward, A. I. \& White, P. C. L. (2004): Monitoring woodland deer populations in the UK: an imprecise science. Mammal Rev. 34(1), 99-114

Sorges, A. (1999): Einfluß des Rotwildes (Cervus elaphus Linné, 1758) auf die Vegetation im Naturpark Nordeifel - Hohes Venn. Zeitschrift für Jagdwissenschaft 45, 250-261

Speidel, G. (1975): Grundlagen und Methoden zur Bestimmung der wirtschaftlich tragbaren Wilddichte beim Schalenwild. Forstarchiv 46

Staines, B. W. \& Welch, D. (1984): Habitat selection of red (Cervus elaphus L.) and roe (Capreolus capreolus) deer in a Sitka spruce plantation. Proceedings of the Royal Society of Edinburgh 82(B), 303-319

Szukiel, E. (1978): Relationship between the extent of bark-peeling and red deer density in relation to prescribed food carrying capacity of a forest. Sylwan 122, $37-43$

Takeuchi, T., Kobayashi, T. \& Nashimoto, M. (2011): Altitudinal differences in bark stripping by sika deer in the subalpine coniferous forest of Mt. Fuji. Forest Ecology and Management 261(11), 2089-2095 
Tamura, N. \& Ohara, S. (2005): Chemical components of hardwood barks stripped by the alien squirrel Callosciurus erythraeus in Japan. Journal of Forest Research 10, 429-433

Tanentzap, A. J., Bazely, D. R., Koh, S., Timciska, M., Haggith, E. G., Carleton, t. J. \& Coomes, D. A. (2011): Seeing the forest for the deer: Do reductions in deer-disturbance lead to forest recovery?, Biological Conservation 144(1), 376-382

Tanentzap, A. J., Kirby, K. J. \& Goldberg, E. (2012): Slow responses of ecosystems to reductions in deer (Cervidae) populations and strategies for achieving recovery. Forest Ecology and Management 264(0), 159-166

The R Development Core Team (1999): The R Environment for Statistical Computing and Graphics - Reference Index, Version 1.6.1 (2002-11-01), http://www.r-project.org

Theuerkauf, J. \& Rouys, S. (2008): Habitat selection by ungulates in relation to predation risk by wolves and humans in the Bialowieza Forest, Poland. Forest Ecology and Management 256(6), 1325-1332

Tjørve, E. (2002): Habitat size and number in multi-habitat landscapes: a model approach based on species-area curves. Ecography 25, 17-24

Trisl, O. (1998): Untersuchungen zur Entwicklung eines optimalen Stichprobenverfahrens für die langfristige Beobachtung der Schälschadensituation. Hainholz Verlag, Göttingen und Braunschweig, 230 Seiten

Trisl, O., Wode, L. \& Akca, A. (1999): Sechs Jahre Schälschadensinventur im Niedersächsischen Forstamt Winnefeld. Forst und Holz 54(15), 425-428

Ueckermann, E. (1956): Untersuchungen über die Ursache des Schälens des Rotwildes. Zeitschrift für Jagdwissenschaft 2, 123-131

Ueckermann, E. (1960): Wildstandsbewirtschaftung und Wildschadenverhütung beim Rotwild. Schriftenreihe der Forschungsstelle für Jagdkunde und Wildschadenverhütung Paul Parey, Hamburg und Berlin 
Ueda, H., Takatsuki, S. \& Takahashi, Y. (2002): Bark stripping of hinoki cypress by sika deer in relation tosnow cover and food availability on Mt Takahara, central Japan. Ecological Research 17, 545-551

Van de Veen, H. E. (1979): Food selection and habitat use in the red deer (Cervus elaphus L.). Thesis. Universität Groningen, Niederlande

Vanik, K. (1979): Influence of mechanical damage on spread of decay in stems of beech. Lesnictvi 25, 349-364

Vasiliauskas, R. (2001): Damage to trees due to forestry operations and its pathological significance in temperate forest: a literature review. Forestry 74(4), 319-336

Vasiliauskas, R. \& Stenlid, J. (1998): Discoloration following bark stripping wounds on Fraxinus excelsior. European Journal of Forest Pathology 28, 383-390

Verheyden, H., Ballon, P., Bernard, V. \& Saint-Andrieux, C. (2006): Variations in bark-stripping by red deer (Cervus elaphus) across Europe. Mammal Rev. $36(3), 217-234$

Völk, F. (1997): Schälschäden und Rotwildmanagement in Abhängigkeit von Jagdgesetz und Waldbau in Österreich. Dissertation. Universität für Bodenkultur, Wien. 252 Seiten

Völk, F. (1998): Schälschäden und Rotwildmanagement in Relation zu Jagdgesetz und Waldaufbau in Österreich. Alpine Umweltprobleme - Ergebnisse des Forschungsprojekts Achenkirch, Teil 34, in: Beiträge zur Umweltgestaltung A, 141. Erich Schmidt Verlag Berlin, 514 Seiten

Völk, F. (1999): Bark peeling frequency in the alpine provinces of Austria: the importance of forest structure and red deer management. Zeitschrift für Jagdwissenschaft 45(1)

Vospernik, S. (2006): Probability of Bark Stripping Damage by Red Deer (Cervus elaphus) in Austria. Silva Fennica 40(4), 589-601

Wagenknecht, E. (1994): Bewirtschaftung von Schalenwild. 6. Auflage. Deutscher Landwirtschaftsverlag, Berlin

Ward, A. (2005): Expanding ranges of wild and feral deer in Great Britain. Mammal Rev. 35(2), 165-173 
Welch, D., Staines, B. W., Scott, D. \& Cart, D. C. (1987): Bark stripping damage by red deer in a Sitka spruce forest in western Scotland I: Incidence. Forestry 60, 249-262

William D. Newmark \& Eric A. Rickart (2012): High-use movement pathways and habitat selection by ungulates. Mammalian Biology - Zeitschrift für Säugetierkunde 77(4), 293-298

Wolf, K.-R. (1994): Untersuchungen zur Biologie der Erdkröte Bufo bufo L. unter besonderer Berücksichtigung des Einflusses von Migrationshindernissen auf das Wanderverhalten und die Entwicklung von vier Erdkrötenpopulationen im Stadtgebiet von Osnabrück. Mellen University Press. Henmoor

Yokoyama, S., Maeji, I., Ueda, T., Ando, M. \& Shibata, E. (2001): Impact of bark stripping by sika deer, Cervus nippon, on subalpine coniferous forests in central Japan. Forest Ecology and Management 140, 93-99 


\section{Erklärung}

Hiermit erkläre ich, dass ich meine Arbeit selbständig und ohne fremde Hilfe angefertigt habe. Die übernommenen Ausführungen anderer Autoren sowie eng an den Gedankengängen Anderer anlehnende eigene Ausführungen habe ich entsprechend gekennzeichnet und die Quellen zitiert.

Philipp Schomaker

August 2014 